\title{
The Catalytic Asymmetric Intermolecular Prins Reaction
}

\author{
C. David Díaz-Oviedo, Rajat Maji, and Benjamin List*
}

Max-Planck-Institut für Kohlenforschung, Kaiser-Wilhelm-Platz 1, 45470 Mülheim an der Ruhr, Germany *E-mail: list@kofo.mpg.de

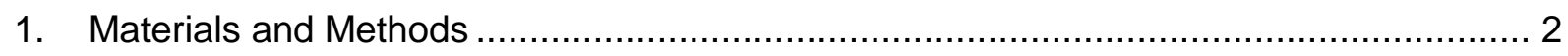

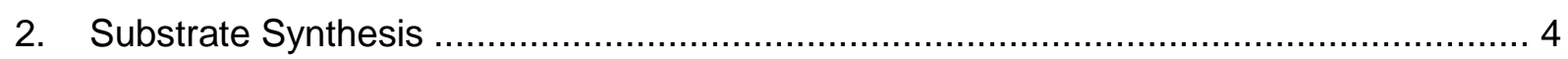

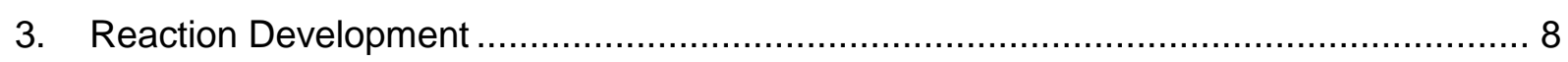

4. ilDP-Catalyzed Intermolecular Prins Reaction .................................................... 10

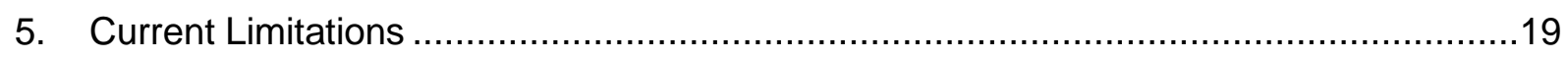

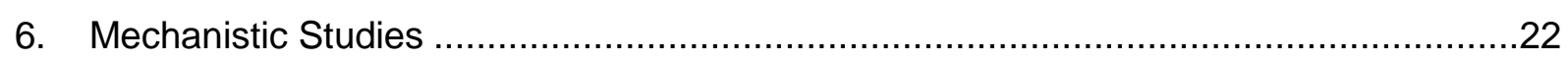

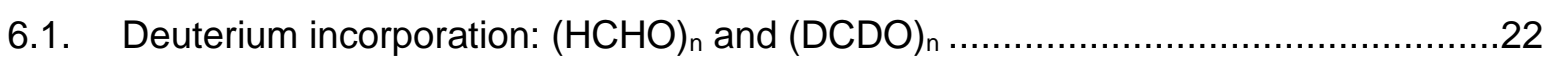

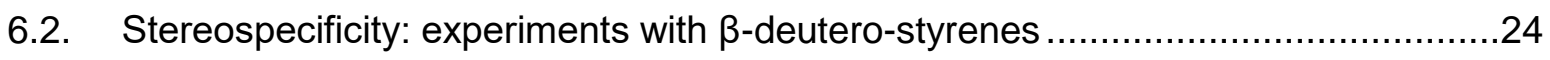

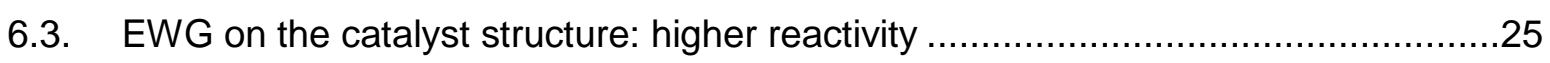

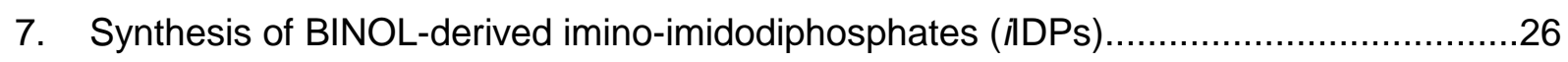

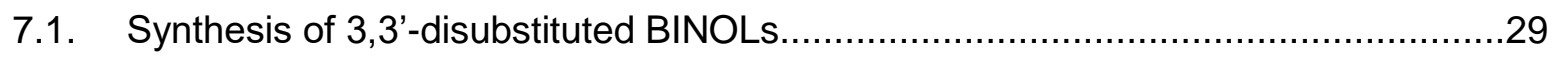

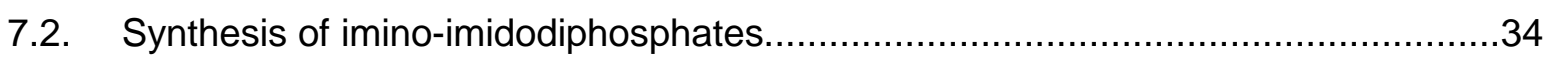

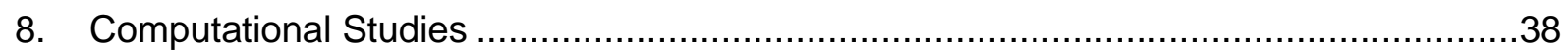

8.1. "Open" vs. "confined" acid: Understanding the "cavity effect" ................................38

8.2. Understanding the Stereoselectivity ................................................................. 41

8.3. Optimized Cartesian Coordinates (PBE-D3/def2-SVP) .....................................43

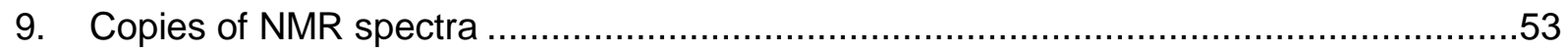

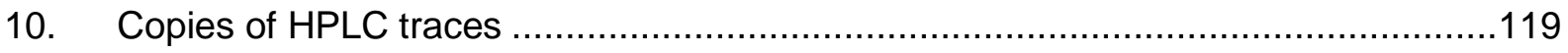

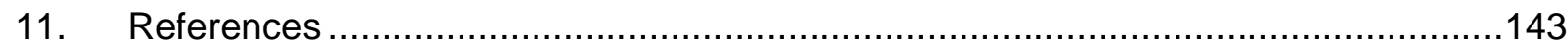




\section{Materials and Methods}

Unless otherwise stated, all reactions were magnetically stirred and conducted in oven-dried $\left(90^{\circ} \mathrm{C}\right)$ or flame-dried glassware in anhydrous solvents under Ar, applying standard Schlenk techniques. Solvents and liquid reagents, as well as solutions of solid or liquid reagents were added via syringes, stainless steel or polyethylene cannulas through rubber septa or through a weak Ar counter-flow. Solid reagents were added through a weak Ar counter-flow. Cooling baths were prepared in Dewar vessels, filled with ice/water $\left(0^{\circ} \mathrm{C}\right)$, cooled acetone $\left(>-78^{\circ} \mathrm{C}\right)$ or dry ice/acetone $\left(-78{ }^{\circ} \mathrm{C}\right)$. Heated oil baths were used for reactions requiring elevated temperatures. Solvents were removed under reduced pressure at $40{ }^{\circ} \mathrm{C}$ using a rotary evaporator, and unless otherwise stated, the remaining compound was dried in high vacuum (10-3 mbar) at ambient temperature. All given yields are isolated yields of chromatographically and NMR spectroscopically pure materials, unless otherwise stated.

Chemicals were purchased from commercial suppliers (including abcr, Acros, Alfa Aesar, Fluorochem, Merck, and $\mathrm{TCl}$ ) and used without further purification unless otherwise stated. $\mathrm{Et}_{3} \mathrm{~N}$ was distilled from $\mathrm{LiAlH}_{4}$ and stored under Ar prior to use. Pyridine was dried and stored over molecular sieves.

Solvents $\left(\mathrm{CyH}, \mathrm{CH}_{2} \mathrm{Cl}_{2}, \mathrm{Et}_{2} \mathrm{O}\right.$, THF, toluene) were dried by distillation from an appropriate drying agent in the technical department of the Max-Planck-Institut für Kohlenforschung and received in Schlenk flasks under Ar. ${ }^{1}$ Other anhydrous solvents were purchased from commercial suppliers and used as received.

Reactions were monitored by thin layer chromatography (TLC) on silica gel pre-coated plastic sheets (0.2 $\mathrm{mm}$, Macherey-Nagel). Visualization was accomplished by irradiation with UV light $(254 \mathrm{~nm}$ and $366 \mathrm{~nm}$ ) and/or phosphomolybdic acid (PMA) stain and/or Cerium Ammonium Molybdate (CAM) stain and/or permanganate stain.

Column chromatography was carried out using Merck silica gel (60 $\AA, 230-400$ mesh, particle size 0.040-0.063 mm) or aluminum oxide (neutral, activated, Brockmann I, Sigma-Aldrich; activity adjustment individually specified) using technical grade solvents. Elution was accelerated using compressed air. Automated column chromatography was conducted on a Biotage $\AA^{\circledR}$ IsoleraTM ISO4SW instrument, using SNAP Ultra HP-SphereTM $25 \mu \mathrm{m}$ chromatography cartridges. All fractions containing a desired substance were combined and concentrated in vacuo, then redissolved in an appropriate solvent and filtered through cotton to remove silica residues.

${ }^{1} \mathrm{H},{ }^{13} \mathrm{C},{ }^{11} \mathrm{~B},{ }^{19} \mathrm{~F},{ }^{31} \mathrm{P}$ nuclear magnetic resonance (NMR) spectra were recorded on a Bruker AV-500, AV-400 or DPX-300 spectrometer in a suitable deuterated solvent. The solvent employed and respective measuring frequency are indicated for each experiment. Chemical shifts are reported with $\mathrm{Me}_{4} \mathrm{Si}$ serving as a universal reference of all nuclides and with two or one digits after the comma. The resonance multiplicity is described as $s$ (singlet), $d$ (doublet), $t$ (triplet), q (quadruplet), $p$ (pentet), hept (heptet), $\mathrm{m}$ (multiplet), and b (broad). All spectra were recorded at $298 \mathrm{~K}$ unless otherwise noted, processed with the program MestReNova 11.0, and coupling constants are reported as observed. The residual deuterated solvent signal relative to $\mathrm{Me}_{4} \mathrm{Si}$ was used as the internal reference in ${ }^{1} \mathrm{H}$ NMR spectra (e.g. $\mathrm{CDCl}_{3}=7.26 \mathrm{ppm}$ ) and are reported as follows: chemical shift in ppm (multiplicity, coupling constant $J$ in $\mathrm{Hz}$, number of protons). ${ }^{11} \mathrm{~B},{ }^{13} \mathrm{C},{ }^{19} \mathrm{~F},{ }^{31} \mathrm{P} N M R$ spectra were referenced according to $\Xi$-values (IUPAC recommendations 2008) ${ }^{2}$ relative to the internal references set in ${ }^{1} \mathrm{H}$ NMR spectra (e.g. ${ }^{13} \mathrm{C}: \mathrm{Me}_{4} \mathrm{Si},{ }^{19} \mathrm{~F}: \mathrm{CCl}_{3} \mathrm{~F},{ }^{31} \mathrm{P}: \mathrm{H}_{3} \mathrm{PO}_{4}$; each $0.00 \mathrm{ppm}$ ). All spectra are broadband decoupled unless otherwise noted.

Electron impact (EI) mass spectrometry (MS) was performed on a Finnigan MAT 8200 (70 eV) or MAT $8400(70 \mathrm{eV})$ spectrometer. Electrospray ionization (ESI) mass spectrometry was conducted on a Bruker ESQ 3000 spectrometer. High resolution mass spectrometry (HRMS) was performed on a Finnigan MAT 95 (EI) or Bruker APEX III FTMS (7T magnet, ESI). The ionization method and mode of 
detection employed is indicated for the respective experiment and all masses are reported in atomic units per elementary charge $(\mathrm{m} / \mathrm{z})$ with an intensity normalized to the most intense peak.

Specific rotations $\left[\alpha_{D}^{T}\right]$ were measured with a Rudolph RA Autopol IV Automatic Polarimeter at the indicated temperature $(T)$ with a sodium lamp (sodium $D$ line, $\lambda=589 \mathrm{~nm}$ ). Measurements were performed in an acid resistant $1 \mathrm{~mL}$ cell $(50 \mathrm{~mm}$ length) with concentrations $(\mathrm{g} /(100 \mathrm{~mL}))$ reported in the corresponding solvent.

High-performance liquid chromatography (HPLC) was performed on Shimadzu LC-20AD liquid chromatograph (SIL-20AC auto sampler, CMB-20A communication bus module, DGU-20A5 degasser, CTO-20AC column oven, SPD-M20A diode array detector), Shimadzu LC-20AB liquid chromatograph (SIL-20ACHT auto sampler, DGU-20A5 degasser, CTO-20AC column oven, SPD-M20A diode array detector), or Shimadzu LC-20AB liquid chromatograph (reversed phase, SIL-20ACHT auto sampler, CTO-20AC column oven, SPD-M20A diode array detector) using Daicel columns with chiral stationary phases. All solvents used were HPLC-grade solvents, purchased from Merck. The column employed and respective solvent mixture are indicated for each experiment.

Gas chromatography (GC) analyses on a chiral stationary phase were performed on HP 6890 and 5890 series instruments (split-mode capillary injection system, flame ionization detector (FID), hydrogen carrier gas). All of these analyses were conducted in the GC department of the Max-PlanckInstitut für Kohlenforschung. The conditions employed are described in detail for the individual experiments.

Liquid chromatography-mass spectrometry (LC-MS) was performed on Shimadzu LC-MS 2020 liquid chromatograph. All solvents used were HPLC-grade solvents purchased from Sigma-Aldrich. The column employed, the respective solvent mixture, and the MS parameters are indicated for each experiment. 


\section{Substrate Synthesis}

Substrates 1a-c, 1f-I and 1n were purchased from Sigma-Aldrich or abcr. Other substrates were prepared by methods indicated below.

\section{General Procedure A: Wittig Olefination}

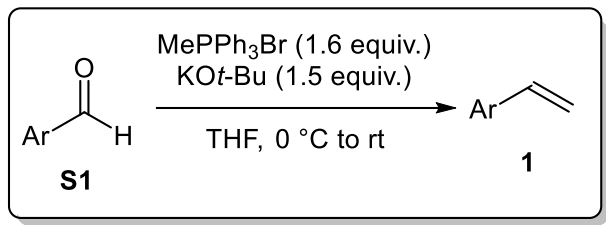

In an oven-dried RB flask, methyltriphenylphosphonium bromide (1.43 g, $4 \mathrm{mmol}, 1.6$ equiv.) was suspended in THF ( $9 \mathrm{~mL}$ ) and cooled to $0^{\circ} \mathrm{C}$ (ice/water bath). In one portion, potassium tert-butoxide (421 mg, $3.75 \mathrm{mmol}, 1.5$ equiv.) was added and the mixture was further stirred vigorously for $30 \mathrm{~min}$. At $0{ }^{\circ} \mathrm{C}$, a solution of the aldehyde $\mathbf{S} 1(2.5 \mathrm{mmol}, 1$ equiv.) in THF $(5 \mathrm{~mL})$ was added dropwise to the formed phosphorus ylide. The mixture was further stirred at $\mathrm{rt}$ for $12 \mathrm{~h}$. After checking full conversion (TLC monitoring), the mixture was diluted with MTBE $(50 \mathrm{~mL})$ and distilled water $(50 \mathrm{~mL})$, and the aqueous layer was extracted with MTBE $(2 \times 30 \mathrm{~mL})$. The combined organic layers were washed with brine $(1 \times 30 \mathrm{~mL})$, dried over anhydrous $\mathrm{Na}_{2} \mathrm{SO}_{4}$, filtered and concentrated under reduced pressure. Purification by flash column chromatography on silica gel ( $n$-pentane $/ \mathrm{CH}_{2} \mathrm{Cl}_{2}$ mixtures) afforded the corresponding olefin 1.

\section{General Procedure B: Aryl Halide Vinylation}

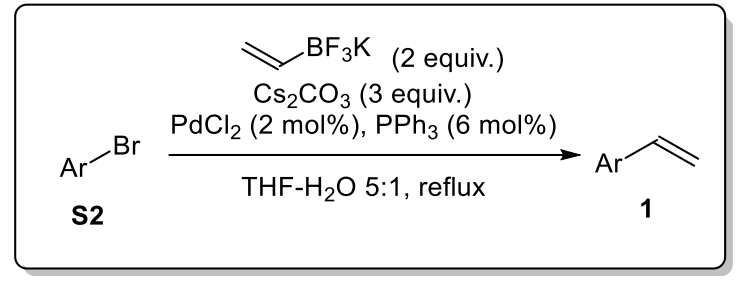

An oven-dried RB flask equipped with a reflux condenser was charged with aryl bromide S2 (2.5 mmol, 1 equiv.) and THF (9.5 mL). To this was added potassium vinyltrifluoroborate $(5 \mathrm{mmol}, 2$ equiv.), $\mathrm{PdCl}_{2}$ (0.05 mmol, $2 \mathrm{~mol} \%$ ), $\mathrm{PPh}_{3}(0.15 \mathrm{mmol}, 6 \mathrm{~mol} \%), \mathrm{Cs}_{2} \mathrm{CO}_{3}(7.5 \mathrm{mmol}, 3$ equiv.), and distilled water $(2.0 \mathrm{~mL})$. The mixture was stirred at $\mathrm{rt}$ and degassed by bubbling Ar for $10 \mathrm{~min}$, then heated to reflux under Ar. After allowing to cool to rt and checking full conversion (TLC monitoring), the mixture was filtered through a short pad of Celite ${ }^{\circledR}$, washing with MTBE $(30 \mathrm{~mL})$. The filtrate was washed with distilled water $(1 \times 30 \mathrm{~mL})$ and brine $(1 \times 30 \mathrm{~mL})$, then dried over anhydrous $\mathrm{Na}_{2} \mathrm{SO}_{4}$, filtered and concentrated under reduced pressure. Purification by flash column chromatography on silica gel ( $n$-pentane/ $\mathrm{CH}_{2} \mathrm{Cl}_{2}$ mixtures) afforded the corresponding olefin $\mathbf{1}$. 


\section{4-vinylphenyl pivalate (1d)}

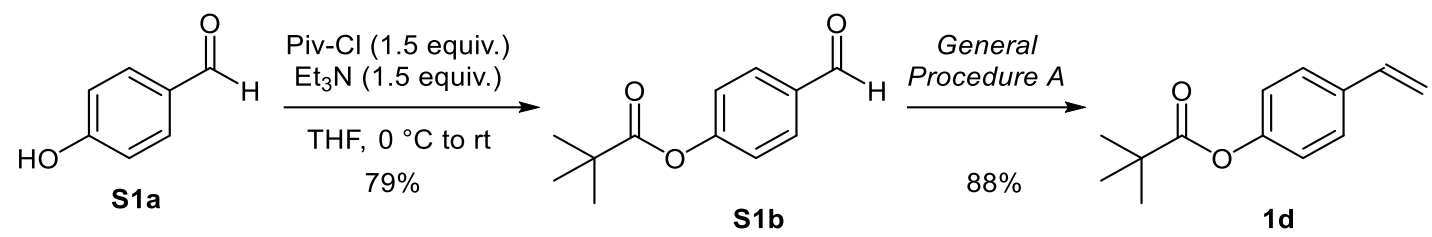

4-formylphenyl pivalate (S1b): (Prepared following a reported procedure ${ }^{3}$ ) In an oven-dried RB flask, 4-hydroxybenzaldehyde S1a (1.22 g, $10 \mathrm{mmol}, 1$ equiv.) was dissolved in THF (10 mL) and cooled to $0{ }^{\circ} \mathrm{C}$ (ice/water bath). Under Ar, triethylamine $(2.1 \mathrm{~mL}, 15 \mathrm{mmol}, 1.5$ equiv.) and pivaloyl chloride (1.8 $\mathrm{mL}, 15 \mathrm{mmol}, 1.5$ equiv., dropwise) were added and the mixture was further stirred at $\mathrm{rt}$ for $2 \mathrm{~h}$. After checking full conversion (TLC monitoring), the mixture was treated with satd. aq. $\mathrm{NH}_{4} \mathrm{Cl}(20 \mathrm{~mL})$ and diluted with MTBE $(30 \mathrm{~mL})$, and the aqueous layer was extracted with MTBE $(3 \times 30 \mathrm{~mL})$. The combined organic layers were washed with brine $(1 \times 30 \mathrm{~mL})$, dried over anhydrous $\mathrm{Na}_{2} \mathrm{SO}_{4}$, filtered and concentrated under reduced pressure. Purification by flash column chromatography on silica gel (i-hexane/EtOAc 95:5 $\rightarrow$ 80:20) afforded the corresponding ester S1b as a white low-melting solid (1.63 g, 79\%).

${ }^{1} \mathrm{H}$ NMR $\left(501 \mathrm{MHz}, \mathrm{CDCl}_{3}\right): \delta 9.99(\mathrm{~s}, 1 \mathrm{H}), 7.91(\mathrm{~d}, J=8.6 \mathrm{~Hz}, 2 \mathrm{H}), 7.24(\mathrm{~d}, J=8.6 \mathrm{~Hz}, 2 \mathrm{H}), 1.37$ (s, $9 \mathrm{H})$. Spectroscopic data was consistent with the values reported in the literature. ${ }^{4}$

4-vinylphenyl pivalate (1d): Following General Procedure A, employing 4-formylphenyl pivalate (S1b, $516 \mathrm{mg}, 2.5 \mathrm{mmol}$ ) as starting material. The crude product was purified by flash column chromatography (eluent: $n$-pentane $/ \mathrm{CH}_{2} \mathrm{Cl}_{2} 90: 10 \rightarrow 70: 30$ ) to give $\mathbf{1 d}$ as a colorless liquid (448 $\mathrm{mg}$, $88 \%)$.

${ }^{1} \mathrm{H}$ NMR $\left(501 \mathrm{MHz}, \mathrm{CDCl}_{3}\right): \delta 7.41(\mathrm{~d}, J=8.5 \mathrm{~Hz}, 2 \mathrm{H}), 7.02(\mathrm{~d}, J=8.6 \mathrm{~Hz}, 2 \mathrm{H}), 6.70$ (dd, $J=17.6$, $10.9 \mathrm{~Hz}, 1 \mathrm{H}), 5.70(\mathrm{dd}, J=17.6,0.9 \mathrm{~Hz}, 1 \mathrm{H}), 5.24(\mathrm{dd}, J=10.9,0.9 \mathrm{~Hz}, 1 \mathrm{H}), 1.36(\mathrm{~s}, 9 \mathrm{H})$.

${ }^{13} \mathrm{C} \mathrm{NMR}\left(126 \mathrm{MHz}, \mathrm{CDCl}_{3}\right): \delta 177.2(\mathrm{C}), 150.8(\mathrm{C}), 136.1(\mathrm{CH}), 135.3(\mathrm{C}), 127.2(\mathrm{CH}), 121.7(\mathrm{CH})$, $114.0\left(\mathrm{CH}_{2}\right), 39.2(\mathrm{C}), 27.3\left(\mathrm{CH}_{3}\right)$.

ESI-HRMS: calculated for $\mathrm{C}_{13} \mathrm{H}_{16} \mathrm{O}_{2} \mathrm{Na}^{+}\left([\mathrm{M}+\mathrm{Na}]^{+}\right): 227.1042$, found: 227.1046 .

\section{S-(4-vinylphenyl) 2,2-dimethylpropanethioate (1e)}

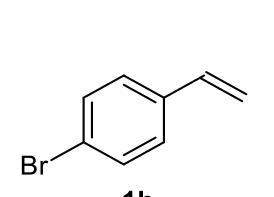

$1 \mathrm{~h}$

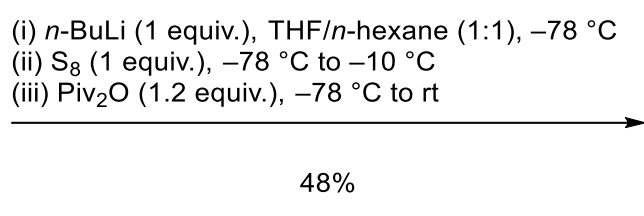

(Adapted from a reported procedure ${ }^{5}$ ): An oven-dried RB flask was charged with 4-bromostyrene (1h, $457 \mathrm{mg}, 2.5 \mathrm{mmol}, 1$ equiv.), dry THF ( $6 \mathrm{~mL})$ and dry $n$-hexane $(6 \mathrm{~mL})$. After cooling the mixture to -78 ${ }^{\circ} \mathrm{C}$, $n$-BuLi (2.5 M in hexanes, $1.0 \mathrm{~mL}, 2.5 \mathrm{mmol}, 1.0$ equiv.) was added dropwise. After stirring at -78 ${ }^{\circ} \mathrm{C}$ for $1.5 \mathrm{~h}$, sulfur powder ( $80 \mathrm{mg}, 2.5 \mathrm{mmol}, 1.0$ equiv.) was added in one portion and the mixture was stirred at $0{ }^{\circ} \mathrm{C}$ for $30 \mathrm{~min}$. Pivalic anhydride $(0.56 \mathrm{~mL}, 2.75 \mathrm{mmol}, 1.1$ equiv.) was added after cooling the reaction mixture again to $-78{ }^{\circ} \mathrm{C}$. After stirring for $1 \mathrm{~h}$ at $-78{ }^{\circ} \mathrm{C}$, the mixture was slowly warmed up to rt and further stirred for $24 \mathrm{~h}$. The reaction was quenched with $10 \mathrm{~mL}$ distilled water and extracted with MTBE $(3 \times 25 \mathrm{~mL})$. Then, the combined organic phase was washed with brine $(1 \times 30$ $\mathrm{mL}$ ), dried over anhydrous $\mathrm{Na}_{2} \mathrm{SO}_{4}$, filtered and concentrated under reduced pressure. Purification by flash column chromatography on silica gel ( $n$-pentane $/ \mathrm{CH}_{2} \mathrm{Cl}_{2}$ 95:5 $\rightarrow$ 70:30) afforded the corresponding thioester 1e as a light-yellow liquid (264 mg, 48\%).

${ }^{1} \mathrm{H}$ NMR $\left(501 \mathrm{MHz}, \mathrm{CDCl}_{3}\right): \delta 7.43(\mathrm{~d}, J=8.4 \mathrm{~Hz}, 2 \mathrm{H}), 7.35(\mathrm{~d}, J=8.4 \mathrm{~Hz}, 2 \mathrm{H}), 6.72(\mathrm{dd}, J=17.5$, $10.9 \mathrm{~Hz}, 1 \mathrm{H}), 5.79(\mathrm{dd}, J=17.6,0.8 \mathrm{~Hz}, 1 \mathrm{H}), 5.31(\mathrm{dd}, J=10.9,0.8 \mathrm{~Hz}, 1 \mathrm{H}), 1.32(\mathrm{~s}, 9 \mathrm{H})$. 
${ }^{13} \mathrm{C}$ NMR (126 MHz, CDCl $)$ : $\delta 204.7(\mathrm{C}), 138.6(\mathrm{C}), 136.3(\mathrm{CH}), 135.2(\mathrm{CH}), 127.5(\mathrm{C}), 127.0(\mathrm{CH})$, $115.3\left(\mathrm{CH}_{2}\right), 47.1(\mathrm{C}), 27.6\left(\mathrm{CH}_{3}\right)$.

ESI-HRMS: calculated for $\mathrm{C}_{13} \mathrm{H}_{16} \mathrm{OSNa}^{+}\left([\mathrm{M}+\mathrm{Na}]^{+}\right)$: 243.0814 , found: 243.0818 .

\section{2-bromo-1-methyl-4-vinylbenzene (1m)}<smiles>C=Cc1ccc(C)c(Br)c1</smiles>

Following General Procedure A, employing 3-bromo-4-methylbenzaldehyde (S1c, 498 mg, 2.5 mmol) as starting material. The crude product was purified by flash column chromatography (eluent: $n$ pentane $/ \mathrm{CH}_{2} \mathrm{Cl}_{2} 100: 0 \rightarrow 70: 30$ ) to give $1 \mathrm{~m}$ as a colorless liquid (396 $\mathrm{mg}, 80 \%$ ).

${ }^{1} \mathrm{H}$ NMR $\left(501 \mathrm{MHz}, \mathrm{CDCl}_{3}\right): \delta 7.58(\mathrm{~d}, J=1.8 \mathrm{~Hz}, 1 \mathrm{H}), 7.24(\mathrm{dd}, J=7.8,1.8 \mathrm{~Hz}, 1 \mathrm{H}), 7.18(\mathrm{~d}, J=7.8$ $\mathrm{Hz}, 1 \mathrm{H}), 6.62(\mathrm{dd}, J=17.6,10.9 \mathrm{~Hz}, 1 \mathrm{H}), 5.71(\mathrm{dd}, J=17.6,0.7 \mathrm{~Hz}, 1 \mathrm{H}), 5.24(\mathrm{~d}, J=10.8 \mathrm{~Hz}, 1 \mathrm{H})$, $2.39(\mathrm{~s}, 3 \mathrm{H})$.

${ }^{13} \mathrm{C} \mathrm{NMR}\left(126 \mathrm{MHz}, \mathrm{CDCl}_{3}\right) \delta 137.4(\mathrm{C}), 137.3(\mathrm{C}), 135.5(\mathrm{CH}), 130.9(\mathrm{CH}), 130.1(\mathrm{CH}), 125.25(\mathrm{C})$, $125.19(\mathrm{CH}), 114.5\left(\mathrm{CH}_{2}\right), 22.8\left(\mathrm{CH}_{3}\right)$. Spectroscopic data was consistent with the values reported in the literature. ${ }^{6}$

APPI-HRMS: calculated for $\mathrm{C}_{9} \mathrm{H}_{10} \mathrm{Br}^{+}\left([\mathrm{M}+\mathrm{H}]^{+}\right): 196.9961$, found: 196.9962 .

\section{2,2-difluoro-5-vinylbenzo[d][1,3]dioxole (10)}

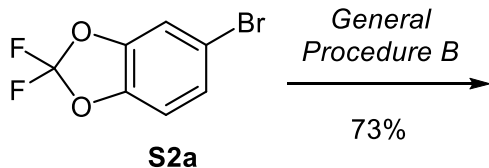<smiles>C=Cc1ccc2c(c1)OC(F)(F)O2</smiles>

Following General Procedure B, employing 5-bromo-2,2-difluoro-1,3-benzodioxole (S2a, 593 mg, 2.5 $\mathrm{mmol}$ ) as starting material. The crude product was purified by flash column chromatography (eluent: $n$ pentane $/ \mathrm{CH}_{2} \mathrm{Cl}_{2} 100: 0 \rightarrow 70: 30$ ) to give 10 as a colorless liquid (337 $\mathrm{mg}, 73 \%$ ).

${ }^{1} \mathrm{H}$ NMR $\left(501 \mathrm{MHz}, \mathrm{CDCl}_{3}\right): \delta 7.15(\mathrm{~d}, J=1.7 \mathrm{~Hz}, 1 \mathrm{H}), 7.07(\mathrm{dd}, J=8.3,1.7 \mathrm{~Hz}, 1 \mathrm{H}), 6.99(\mathrm{~d}, J=8.2$ $\mathrm{Hz}, 1 \mathrm{H}), 6.66$ (dd, $J=17.5,10.9 \mathrm{~Hz}, 1 \mathrm{H}), 5.66(\mathrm{~d}, J=17.5 \mathrm{~Hz}, 1 \mathrm{H}), 5.25(\mathrm{~d}, J=10.9 \mathrm{~Hz}, 1 \mathrm{H})$.

$\left.{ }^{19} \mathrm{~F} \mathrm{NMR} \mathrm{(471} \mathrm{MHz,} \mathrm{CDCl}_{3}\right): \delta-50.24(\mathrm{~s}, 2 \mathrm{~F})$.

${ }^{13} \mathrm{C} \mathrm{NMR}\left(126 \mathrm{MHz}, \mathrm{CDCl}_{3}\right): \delta 144.4(\mathrm{C}), 143.4(\mathrm{C}), 135.7(\mathrm{CH}), 134.3(\mathrm{C}), 131.8$ (C, t, J=255.1 Hz), $122.5(\mathrm{CH}), 114.3\left(\mathrm{CH}_{2}\right), 109.4(\mathrm{CH}), 106.6(\mathrm{CH})$. Spectroscopic data was consistent with the values reported in the literature. ${ }^{7}$

APPI-HRMS: calculated for $\mathrm{C}_{9} \mathrm{H}_{7} \mathrm{~F}_{2} \mathrm{O}_{2}+\left([\mathrm{M}+\mathrm{H}]^{+}\right):$185.0409, found: 185.0410 .

\section{1-allyl-4-vinylbenzene (1p)}

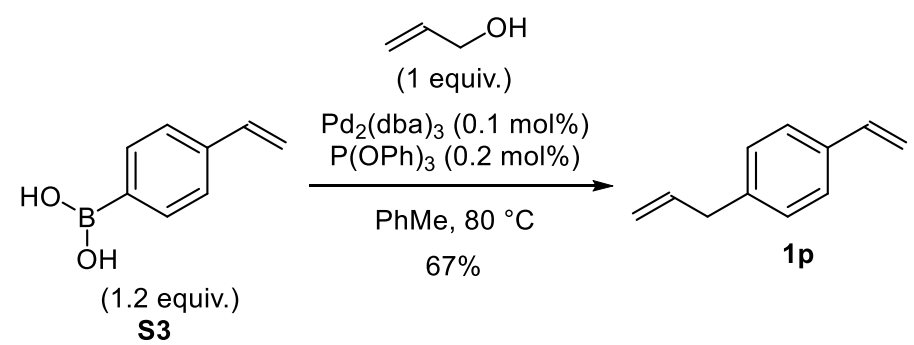

(Adapted from a reported procedure $\left.{ }^{8}\right)$ : A two-necked RB flask under Ar atmosphere was charged with $\mathrm{Pd}_{2}(\mathrm{dba})_{3}$ (4.6 mg, $5 \mu \mathrm{mol}, 0.1 \mathrm{~mol} \%$ ), 4-vinylphenyl boronic acid (S3, $888 \mathrm{mg}, 6 \mathrm{mmol}, 1.2$ equiv.) and 1,4-dioxane $(6.5 \mathrm{~mL})$. Triphenylphosphite $(2.5 \mu \mathrm{L}, 10 \mu \mathrm{mol}, 0.2 \mathrm{~mol} \%)$ and allyl alcohol $(290 \mathrm{mg}, 5$ mmol, 1 equiv.) were added and the mixture was heated at $80^{\circ} \mathrm{C}$ (oil bath) for $6 \mathrm{~h}$. After cooling to rt, 
the reaction mixture was diluted with MTBE $(50 \mathrm{~mL})$ and washed with brine $(1 \times 30 \mathrm{~mL})$, dried over anhydrous $\mathrm{Na}_{2} \mathrm{SO}_{4}$, filtered and concentrated under reduced pressure. Purification by flash column chromatography on silica gel ( $n$-pentane) afforded the corresponding olefin $1 p$ as a colorless liquid (480 mg, 67\%).

${ }^{1} \mathrm{H}$ NMR $\left(501 \mathrm{MHz}, \mathrm{CD}_{2} \mathrm{Cl}_{2}\right): \delta 7.35(\mathrm{~d}, J=8.1 \mathrm{~Hz}, 2 \mathrm{H}), 7.16(\mathrm{~d}, J=8.2 \mathrm{~Hz}, 2 \mathrm{H}), 6.71(\mathrm{dd}, J=17.6$, $10.9 \mathrm{~Hz}, 1 \mathrm{H}$ ), 5.97 (ddt, $J=16.9,10.1,6.7 \mathrm{~Hz}, 1 \mathrm{H}$ ), 5.72 (dd, $J=17.6,1.0 \mathrm{~Hz}, 1 \mathrm{H}$ ), 5.20 (dd, $J=10.9$, $1.0 \mathrm{~Hz}, 1 \mathrm{H}), 5.13-5.03(\mathrm{~m}, 2 \mathrm{H}), 3.38(\mathrm{~d}, J=6.8 \mathrm{~Hz}, 2 \mathrm{H})$.

${ }^{13} \mathrm{C} \mathrm{NMR}\left(126 \mathrm{MHz}, \mathrm{CD}_{2} \mathrm{Cl}_{2}\right)$ : $\delta 140.4(\mathrm{C}), 137.9(\mathrm{CH}), 137.0(\mathrm{CH}), 135.9(\mathrm{C}), 129.1(\mathrm{CH}), 126.6(\mathrm{CH})$, $115.9\left(\mathrm{CH}_{2}\right), 113.3\left(\mathrm{CH}_{2}\right), 40.3\left(\mathrm{CH}_{2}\right)$. Spectroscopic data was consistent with the values reported in the literature. ${ }^{9}$

\section{Styrene- $\beta, \beta-d_{2}\left(1 a^{\prime}\right)$}

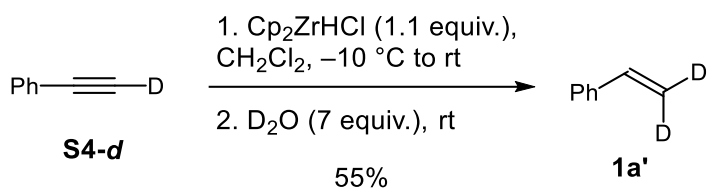

(Adapted from a reported procedure ${ }^{10}$ ): A two-necked RB flask under $\mathrm{Ar}$ atmosphere was charged with phenylacetylene- $d(\mathbf{S} 4-\boldsymbol{d})^{10}(1.99 \mathrm{~g}, 19.5 \mathrm{mmol})$ and dry $\mathrm{CH}_{2} \mathrm{Cl}_{2}(50 \mathrm{~mL})$. The flask was covered with aluminum foil and the mixture was cooled to $-10^{\circ} \mathrm{C}$. Schwartz's Reagent $(5.53 \mathrm{~g}, 21.5 \mathrm{mmol}, 1.1$ equiv.) was then added in three equal portions in rapid succession (over $2 \mathrm{~min}$ ). The mixture was allowed to stir at $-10^{\circ} \mathrm{C}$ for $15 \mathrm{~min}$, then the cold bath was removed and the stirring was continued at $\mathrm{rt}$ in the dark for $4 \mathrm{~h}$. The flask was cooled to $0{ }^{\circ} \mathrm{C}$, and the mixture was quenched with $\mathrm{D}_{2} \mathrm{O}(2.5 \mathrm{~mL}$, $99.9 \% \mathrm{D}, 136.5 \mathrm{mmol}, 7$ equiv.) and stirred vigorously at $\mathrm{rt}$ for $12 \mathrm{~h}$. The mixture was diluted with $\mathrm{CH}_{2} \mathrm{Cl}_{2}\left(50 \mathrm{~mL}\right.$ ), followed by the addition of anhydrous $\mathrm{Na}_{2} \mathrm{SO}_{4}$ and filtration, washing with $\mathrm{CH}_{2} \mathrm{Cl}_{2}$. The filtrate was concentrated under reduced pressure ( 400 mbar, water bath of rotavap at $25{ }^{\circ} \mathrm{C}$; no heating, product is volatile) until $5-10 \mathrm{~mL}$ remained. $n$-Pentane $(50 \mathrm{~mL})$ was added and the mixture was filtered over a Celite ${ }^{\circledR}$ pad to remove the white precipitate; the filter cake was rinsed with $n$ pentane and the filtrate was again concentrated under reduced pressure (400 mbar, $25{ }^{\circ} \mathrm{C}$ ). Purification by flash column chromatography on silica gel ( $n$-pentane; removal of solvent on rotavap at 400 mbar, $25^{\circ} \mathrm{C}$ ) afforded the corresponding olefin 1a' as a colorless liquid (1.13 g, 55\%). Approx. $97 \%$ D-incorporation.

${ }^{1} \mathrm{H}$ NMR (501 MHz, CDCl 3 ) $\delta 7.45-7.40(\mathrm{~m}, 2 \mathrm{H}), 7.37-7.31(\mathrm{~m}, 2 \mathrm{H}), 7.26$ (tt, $\left.J=6.7,1.3 \mathrm{~Hz}, 1 \mathrm{H}\right)$, $6.72(\mathrm{~d}, J=2.8 \mathrm{~Hz}, 1 \mathrm{H})$. Spectroscopic data was consistent with the values reported in the literature. ${ }^{11}$

trans-Styrene-( $\beta)-d(\mathbf{1 q})$ and cis-Styrene-( $\beta)-d(1 \mathbf{r})$

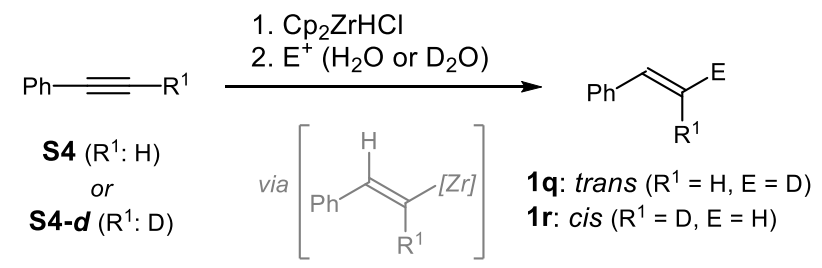

(Adapted from a reported procedure ${ }^{10}$ ): Prepared in a similar way to 1a', choosing between starting alkyne (S4 or S4- $\boldsymbol{d}$ ) and electrophile quench $\left(\mathrm{H}_{2} \mathrm{O}\right.$ or $\left.\mathrm{D}_{2} \mathrm{O}\right)$.

Spectroscopic data was consistent with the values reported in the literature (trans-olefin ${ }^{12}$ and cisolefin $\left.{ }^{10}\right)$. 


\section{Reaction Development}

At the onset of our studies, we tested the reaction of styrene (1a) with paraformaldehyde (2a) using as catalysts several chiral Brønsted acids available at that moment in our laboratory (Table S-1).

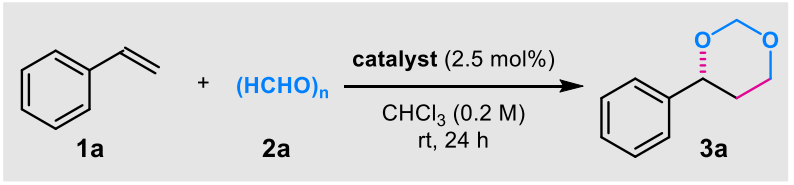

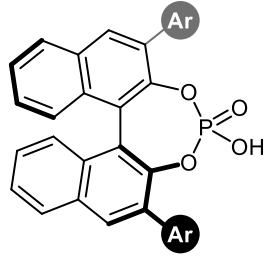

$\mathrm{Ar}=4-\mathrm{t}-\mathrm{Bu}-\mathrm{C}_{6} \mathrm{H}_{4}$, or $\mathrm{Ar}=3,5-\left(\mathrm{CF}_{3}\right)_{2} \mathrm{C}_{6} \mathrm{H}_{3}$, CPA no conversion

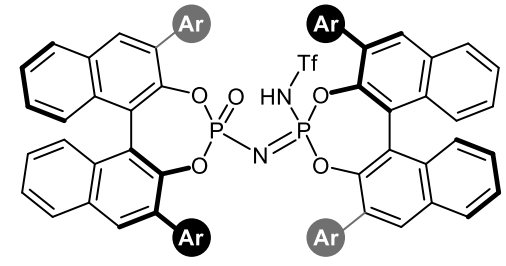

$\mathrm{Ar}=2-i-\mathrm{Pr}-5-\mathrm{Me}-\mathrm{C}_{6} \mathrm{H}_{3}$ iIDP $2 \%$ yield $(\mathrm{er}=91: 9)$<smiles></smiles>

$\mathrm{Ar}=3,5-\left(\mathrm{CF}_{3}\right)_{2} \mathrm{C}_{6} \mathrm{H}_{3}$

BINSA

$72 \%$ yield $(\mathrm{er}=47: 53)$

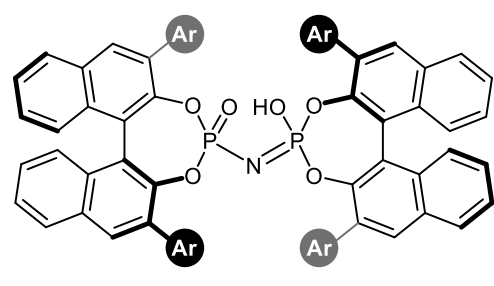

$\mathrm{Ar}=2,4,6-\mathrm{Et}_{3}-\mathrm{C}_{6} \mathrm{H}_{2}$

IDP

no conversion

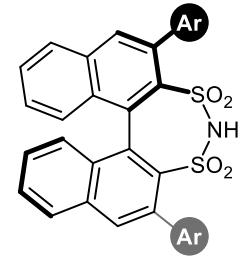

$\mathrm{Ar}=3,5-\left(\mathrm{CF}_{3}\right)_{2} \mathrm{C}_{6} \mathrm{H}_{3}$

DSI

no conversion

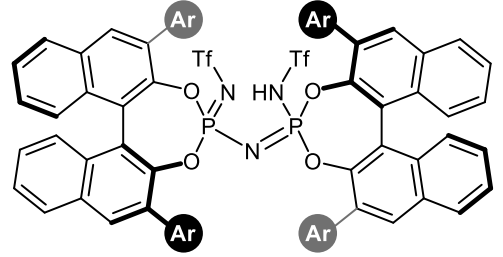

$\mathrm{Ar}=\mathrm{Ph}$

IDPi

$57 \%$ yield $($ er $=56: 44)$

a $0.025 \mathrm{mmol}$ 1a, 3 equiv. 2a, 5 mol\% catalyst, in $\mathrm{CHCl}_{3}(125 \mu \mathrm{L})$ at $\mathrm{rt}$ for $48 \mathrm{~h}$. Yields were determined by ${ }^{1} \mathrm{H}$ NMR analysis using $\mathrm{Ph}_{3} \mathrm{CH}$ as internal standard. Enantiomeric ratios were determined by HPLC analysis with a chiral stationary phase.

Using the confined imino-imidodiphosphate (ilDP) $\mathbf{4 a}$, which was an optimal catalyst for the Prins cyclization, ${ }^{13}$ resulted in this case only in traces of product, yet with good enantioselectivity ( $82 \%$ ee). A more acidic, confined imidodiphosphorimidate (IDPi), as expected, provided the product in better yield $(57 \%)$, but with a significant decrease in enantioselectivity (12\% ee).

Therefore, we dedicated our efforts to increase the yield of the process by making a more acidic iDP catalyst, but without modifying the inner active site. Introducing electron-withdrawing groups in the aryl substituent (at the 3,3' positions) increased the reactivity: catalyst $\mathbf{S 1 1}$ (prepared in our laboratory for another project, which will be reported in due course) afforded 3a in $24 \%$ yield after $24 \mathrm{~h}$ at rt, although with decreased enantioselectivity $(79.5: 20.5$ er). Further exploration led to catalyst $\mathbf{4 b}$, with which (2.5 mol\%), the product was obtained in $74 \%$ and promising $91.5: 8.5 \mathrm{er}$.

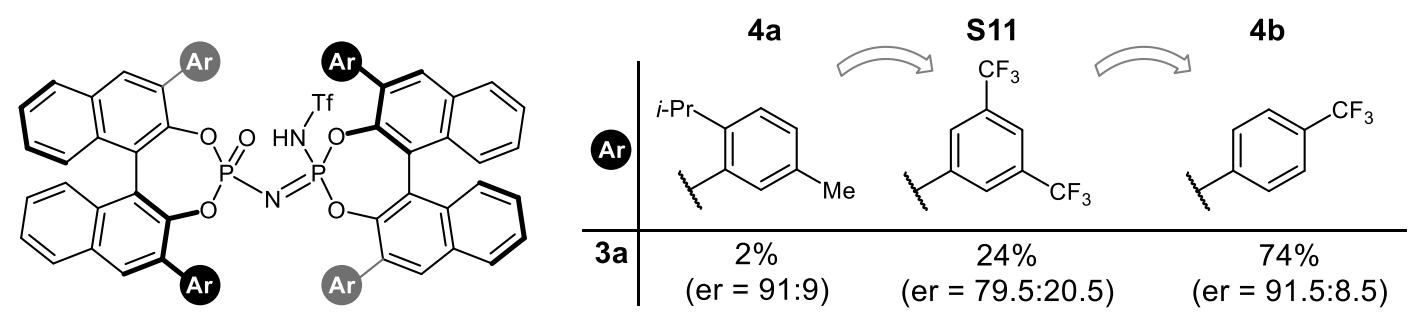


The Catalytic Asymmetric Intermolecular Prins Reaction Reaction Development

Table S-2. Reaction development for the ilDP-catalyzed Prins reaction of styrene (1a) and paraformaldehyde (2a).

\begin{tabular}{c|c|c|c|c|c||c|c} 
entry & $\begin{array}{c}\text { temperature } \\
\left({ }^{\circ} \mathrm{C}\right)\end{array}$ & solvent & equiv. 2a & $\begin{array}{c}\text { concentration } \\
(\mathrm{M})\end{array}$ & $\begin{array}{c}\text { cat. loading } \\
(\mathrm{mol} \%)\end{array}$ & \%yield 3a & er 3a \\
\hline 1 & 50 & $\mathrm{CHCl}_{3}$ & 2.5 & 0.2 & 2.5 & 85 & $89: 11$ \\
2 & 25 & $\mathrm{CHCl}_{3}$ & 2.5 & 0.2 & 2.5 & 75 & $91.5: 8.5$ \\
3 & 0 & $\mathrm{CHCl}_{3}$ & 2.5 & 0.2 & 2.5 & 5 & $93.5: 6.5$ \\
\hline 4 & 25 & $\mathrm{CH}_{2} \mathrm{Cl}_{2}$ & 2.5 & 0.2 & 2.5 & 70 & $88: 12$ \\
5 & 25 & $\mathrm{Et}_{2} \mathrm{O}$ & 2.5 & 0.2 & 2.5 & 3 & $85.5: 14.5$ \\
6 & 25 & $\mathrm{PhMe}_{7}$ & 2.5 & 0.2 & 2.5 & 61 & $88: 12$ \\
8 & 25 & $\mathrm{PhH}$ & 2.5 & 0.2 & 2.5 & 50 & $89.5: 10.5$ \\
9 & 25 & $\mathrm{MeCy}$ & 2.5 & 0.2 & 2.5 & 41 & $94: 6$ \\
\hline 10 & 25 & $\mathrm{CyH}$ & 2.5 & 0.2 & 2.5 & 63 & $94.5: 5.5$ \\
\hline 11 & 25 & $\mathrm{CyH}$ & 6 & 0.2 & 2.5 & 64 & $94: 6$ \\
12 & 25 & $\mathrm{CyH}$ & 2.5 & 0.1 & 2.5 & 50 & $94: 6$ \\
13 & 25 & $\mathrm{CyH}$ & 2.5 & 0.05 & 2.5 & 28 & $94.5: 5.5$ \\
\hline 14 & 25 & $\mathrm{CyH}$ & 2.5 & 0.5 & 2.5 & 69 & $92: 8$ \\
15 & 25 & $\mathrm{CyH}$ & 2.5 & 0.1 & 5.0 & 72 & $93: 7$ \\
& & $\mathrm{CyH}$ & 2.5 & 0.1 & 1.0 & 29 & $94.5: 5.5$
\end{tabular}

As can be extracted from Table S-2, performing the reaction at $\mathrm{rt}\left(25^{\circ} \mathrm{C}\right)$, with cyclohexane as solvent, turned out to be the best conditions from the tested ones toward good enantioselectivity.

The absolute configuration of the produced 1,3-dioxane (3a) was determined by HPLC analysis, comparing with the retention times of pure enantiomers, which were prepared by acetalization of the commercially available enantiomers of 1 -phenylpropane-1,3-diol. ${ }^{14}$ Thus, when catalyst $(S, S)-4 \mathbf{b}$ is used, the major enantiomer of the Prins product 3a matches on HPLC with the $(R)$-1,3-dioxane.<smiles>OCC[C@@H](O)c1ccccc1</smiles>

$(R)-5$

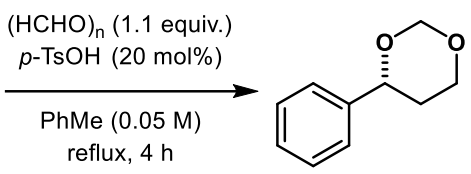

(R)-3a

$70 \%$ $\mathrm{er}=98: 2$<smiles>c1ccc(C2CCOCO2)cc1</smiles>

(S)-3a

$62 \%$

er $=4: 96$

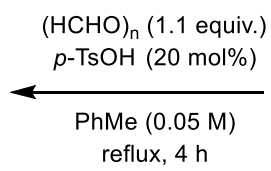<smiles>OCCC(O)c1ccccc1</smiles>

(S)-5 


\section{IDP-Catalyzed Intermolecular Prins Reaction}

General Procedure C: ilDP-Catalyzed Prins reaction of aryl olefins and paraformaldehyde

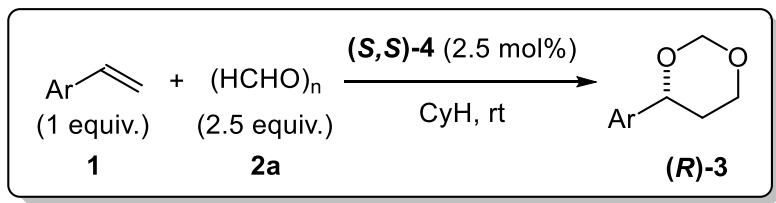

An oven-dried glass vial was charged with paraformaldehyde 2 a (18.8 mg, $0.625 \mathrm{mmol}, 2.5$ equiv.), $(S, S)$-ilDP catalyst $4(62.5 \mu \mathrm{mol}, 2.5 \mathrm{~mol} \%)$, dry cyclohexane (depending on the concentration, indicated in each case), and olefin 1 ( $0.25 \mathrm{mmol}, 1$ equiv.) under Ar. Then, the mixture was vigorously stirred at $\mathrm{rt}$ for a certain time (indicated in each case). The reaction was stopped by adding distilled water $(10 \mathrm{~mL})$, followed by extraction with MTBE $(3 \times 20 \mathrm{~mL})$. The combined organic layers were washed with brine $(1 \times 10 \mathrm{~mL})$, then dried over anhydrous $\mathrm{Na}_{2} \mathrm{SO}_{4}$, filtered and concentrated under reduced pressure. Purification by flash column chromatography on silica gel (n-pentane/MTBE mixtures) afforded the corresponding enantioenriched 4-substituted-1,3-dioxane product 3.

(for the synthesis of racemates, see General Procedure $D$ at the end of this section)

\section{(R)-4-phenyl-1,3-dioxane (3a)}

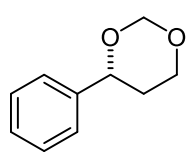

Prepared following General Procedure C, from styrene (1a, $29 \mu \mathrm{L}, 0.25 \mathrm{mmol})$, paraformaldehyde 2a (18.8 mg, $0.625 \mathrm{mmol}, 2.5$ equiv.), with catalyst $(S, S)-4 \mathrm{c}$ (10.6 $\mathrm{mg}, 62.5 \mu \mathrm{mol}, 2.5 \mathrm{~mol} \%)$, in dry cyclohexane $(2.5 \mathrm{~mL}, 0.1 \mathrm{M})$ for $72 \mathrm{~h}$. Purification by flash column chromatography (eluent: $n$-pentane/MTBE 100:0 $\rightarrow$ 95:5) to give 3 a as a colorless liquid $(36.4 \mathrm{mg}, 89 \%$, er $=95.5: 4.5)$.

${ }^{1} \mathrm{H}$ NMR $\left(501 \mathrm{MHz}, \mathrm{CDCl}_{3}\right) \delta 7.40-7.35(\mathrm{~m}, 4 \mathrm{H}), 7.32-7.28(\mathrm{~m}, 1 \mathrm{H}), 5.23(\mathrm{~d}, J=6.3 \mathrm{~Hz}, 1 \mathrm{H}), 4.91$ (d, $J=6.4 \mathrm{~Hz}, 1 \mathrm{H}), 4.66$ (dd, $J=11.3,2.5 \mathrm{~Hz}, 1 \mathrm{H}), 4.21$ (dd, $J=11.5,4.9 \mathrm{~Hz}, 1 \mathrm{H}$ ), 3.89 (td, $J=11.9$, $2.5 \mathrm{~Hz}, 1 \mathrm{H}$ ), 2.11 (dddd, $J=13.5,12.3,11.3,4.9 \mathrm{~Hz}, 1 \mathrm{H}), 1.76-1.70(\mathrm{~m}, 1 \mathrm{H})$.

${ }^{13} \mathrm{C} \mathrm{NMR}\left(126 \mathrm{MHz}, \mathrm{CDCl}_{3}\right) \delta 141.6(\mathrm{C}), 128.6(\mathrm{CH}), 128.0(\mathrm{CH}), 125.9(\mathrm{CH}), 94.3\left(\mathrm{CH}_{2}\right), 78.9(\mathrm{CH})$, $67.1\left(\mathrm{CH}_{2}\right), 34.1\left(\mathrm{CH}_{2}\right)$. Spectroscopic data was consistent with the values reported in the literature. ${ }^{15}$

ESI-HRMS: calculated for $\mathrm{C}_{10} \mathrm{H}_{12} \mathrm{O}_{2} \mathrm{Na}^{+}\left([\mathrm{M}+\mathrm{Na}]^{+}\right): 187.0729$, found: 187.0732 .

$\left[\alpha_{D}^{25}\right]=+32.1\left(c=0.746, \mathrm{CHCl}_{3}\right)$.

HPLC (Chiralpak IB-3 column, Heptane/i-PrOH 95:5, $1 \mathrm{~mL} / \mathrm{min}, 25^{\circ} \mathrm{C}, 220 \mathrm{~nm}$ ): $\mathrm{t}_{\mathrm{R}}=3.6 \mathrm{~min}$ (major), $4.5 \mathrm{~min}$ (minor).

Racemate synthesis: Following General Procedure D (condition A).

\section{(R)-4-(4-(chloromethyl)phenyl)-1,3-dioxane (3b)}

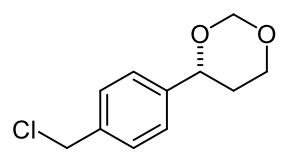

Prepared following General Procedure $C$, from 4-vinylbenzyl chloride (1b, $35 \mu \mathrm{L}$, $0.25 \mathrm{mmol}$ ), paraformaldehyde $2 \mathrm{a}$ ( $18.8 \mathrm{mg}, 0.625 \mathrm{mmol}, 2.5$ equiv.), with catalyst $(S, S)-4 b$ (8.6 mg, $62.5 \mu \mathrm{mol}, 2.5 \mathrm{~mol} \%)$, in dry cyclohexane (1.25 mL, $0.2 \mathrm{M})$ for 5 days. Purification by flash column chromatography (eluent: $n$-pentane/MTBE $100: 0 \rightarrow 90: 10)$ to give $\mathbf{3 b}$ as a white solid $(30.1 \mathrm{mg}, 57 \%$, er $=95.5: 4.5)$.

${ }^{1} \mathrm{H}$ NMR $\left(501 \mathrm{MHz}, \mathrm{CDCl}_{3}\right) \delta 7.39(\mathrm{~d}, J=8.4 \mathrm{~Hz}, 2 \mathrm{H}), 7.37(\mathrm{~d}, J=8.4 \mathrm{~Hz}, 2 \mathrm{H}), 5.22(\mathrm{~d}, J=6.4 \mathrm{~Hz}$, $1 \mathrm{H}), 4.90(\mathrm{~d}, J=6.4 \mathrm{~Hz}, 1 \mathrm{H}), 4.66(\mathrm{dd}, J=11.3,2.6 \mathrm{~Hz}, 1 \mathrm{H}), 4.59(\mathrm{~s}, 2 \mathrm{H}), 4.21$ (dd, $J=11.5,4.9 \mathrm{~Hz}$, $1 \mathrm{H}$ ), 3.88 (td, $J=11.8,2.4 \mathrm{~Hz}, 1 \mathrm{H}), 2.07$ (dddd, $J=13.4,12.2,11.3,4.8 \mathrm{~Hz}, 1 \mathrm{H}), 1.76-1.69(\mathrm{~m}, 1 \mathrm{H})$. ${ }^{13} \mathrm{C} \mathrm{NMR}\left(126 \mathrm{MHz}, \mathrm{CDCl}_{3}\right) \delta 141.9(\mathrm{C}), 137.1(\mathrm{C}), 128.9(\mathrm{CH}), 126.3(\mathrm{CH}), 94.3\left(\mathrm{CH}_{2}\right), 78.4(\mathrm{CH})$, $67.0\left(\mathrm{CH}_{2}\right), 46.1\left(\mathrm{CH}_{2}\right), 34.1\left(\mathrm{CH}_{2}\right)$.

${ }^{13} \mathrm{C} \mathrm{NMR}\left(126 \mathrm{MHz}, \mathrm{CDCl}_{3}\right) \delta$ 128.89, 126.26, 94.30, 78.46, 67.00, 46.09, 34.08.

ESI-HRMS: calculated for $\mathrm{C}_{11} \mathrm{H}_{13} \mathrm{ClO}_{2} \mathrm{Na}^{+}\left([\mathrm{M}+\mathrm{Na}]^{+}\right)$: 235.0496 , found: 235.0498 . 
$\left[\alpha_{D}^{25}\right]=+47.1\left(c=0.255, \mathrm{CHCl}_{3}\right)$.

HPLC (Chiralpak IC-3 column, Heptane/i-PrOH 98:2, $1 \mathrm{~mL} / \mathrm{min}, 25^{\circ} \mathrm{C}, 220 \mathrm{~nm}$ ): $\mathrm{t}_{\mathrm{R}}=7.0 \mathrm{~min}$ (minor), 8.0 min (major).

Racemate synthesis: Following General Procedure D (condition C).

\section{(R)-4-(4-(tert-butyl)phenyl)-1,3-dioxane (3c)}

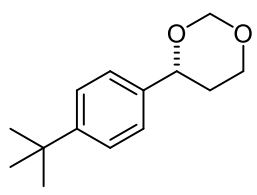

Prepared following General Procedure $C$, from 4-tert-butylstyrene (1c, $40 \mu \mathrm{L}, 0.25$ $\mathrm{mmol})$, paraformaldehyde $2 \mathrm{a}(18.8 \mathrm{mg}, 0.625 \mathrm{mmol}, 2.5$ equiv.), with catalyst $(S, S)$ 4b $(8.6 \mathrm{mg}, 62.5 \mu \mathrm{mol}, 2.5 \mathrm{~mol} \%)$, in dry cyclohexane $(5 \mathrm{~mL}, 0.05 \mathrm{M})$ for $72 \mathrm{~h}$. Purification by flash column chromatography (eluent: $n$-pentane/MTBE 100:0 $\rightarrow$ 95:5) to give $3 \mathrm{c}$ as a white solid $(49.1 \mathrm{mg}, 89 \%$, er $=91.5: 8.5)$.

${ }^{1} \mathrm{H}$ NMR $\left(501 \mathrm{MHz}, \mathrm{CDCl}_{3}\right) \delta 7.39(\mathrm{~d}, J=8.4 \mathrm{~Hz}, 2 \mathrm{H}), 7.31(\mathrm{~d}, J=8.2 \mathrm{~Hz}, 2 \mathrm{H}), 5.21(\mathrm{~d}, J=6.3 \mathrm{~Hz}$, $1 \mathrm{H}), 4.90(\mathrm{~d}, J=6.3 \mathrm{~Hz}, 1 \mathrm{H}), 4.63(\mathrm{dd}, J=11.3,2.5 \mathrm{~Hz}, 1 \mathrm{H}), 4.21$ (dd, $J=11.4,4.8 \mathrm{~Hz}, 1 \mathrm{H}$ ), 3.88 (td, $J=11.9,2.5 \mathrm{~Hz}, 1 \mathrm{H}$ ), 2.14 (dddd, $J=13.4,12.2,11.3,4.8 \mathrm{~Hz}, 1 \mathrm{H}), 1.76-1.68(\mathrm{~m}, 1 \mathrm{H}), 1.32(\mathrm{~s}, 9 \mathrm{H})$.

${ }^{13} \mathrm{C}$ NMR $\left(126 \mathrm{MHz}, \mathrm{CDCl}_{3}\right) \delta 151.0(\mathrm{C}), 138.5(\mathrm{C}), 125.8(\mathrm{CH}), 125.5(\mathrm{CH}), 94.4\left(\mathrm{CH}_{2}\right), 78.8(\mathrm{CH})$, $67.1\left(\mathrm{CH}_{2}\right), 34.7(\mathrm{C}), 33.8\left(\mathrm{CH}_{2}\right), 31.5\left(\mathrm{CH}_{3}\right)$. Spectroscopic data was consistent with the values reported in the literature. ${ }^{15}$

ESI-HRMS: calculated for $\mathrm{C}_{14} \mathrm{H}_{20} \mathrm{O}_{2} \mathrm{Na}^{+}\left([\mathrm{M}+\mathrm{Naa}]^{+}\right): 243.1355$, found: 243.1358 .

$\left[\alpha_{D}^{25}\right]=+29.8\left(c=0.450, \mathrm{CHCl}_{3}\right)$.

HPLC (Chiralpak AD-3 column, Heptane/ $i-\mathrm{PrOH} 97: 3,0.5 \mathrm{~mL} / \mathrm{min}, 25^{\circ} \mathrm{C}, 220 \mathrm{~nm}$ ): $\mathrm{t}_{\mathrm{R}}=6.2 \mathrm{~min}$ (major), 6.9 min (minor).

Racemate synthesis: Following General Procedure $D$ (condition A).

(R)-4-(1,3-dioxan-4-yl)phenyl pivalate (3d)

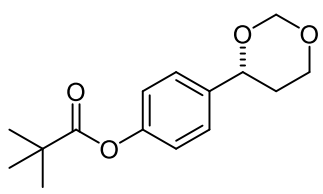

Prepared following General Procedure $C$, from 4-vinylphenyl pivalate (1d, 52 $\mu \mathrm{L}, 0.25 \mathrm{mmol})$, paraformaldehyde $2 \mathrm{a}(18.8 \mathrm{mg}, 0.625 \mathrm{mmol}, 2.5$ equiv.), with catalyst $(S, S)-4 e(12.4 \mathrm{mg}, 62.5 \mu \mathrm{mol}, 2.5 \mathrm{~mol} \%)$, in dry cyclohexane $(2.5 \mathrm{~mL}$, $0.1 \mathrm{M})$ for $72 \mathrm{~h}$. Purification by flash column chromatography (eluent: $n$ pentane/MTBE 100:0 $\rightarrow$ 80:20) to give $3 \mathbf{d}$ as a white solid $(54.9 \mathrm{mg}, 83 \%$, er $=91: 9$ ).

${ }^{1} \mathrm{H}$ NMR $\left(501 \mathrm{MHz}, \mathrm{CDCl}_{3}\right) \delta 7.38(\mathrm{~d}, J=8.5 \mathrm{~Hz}, 2 \mathrm{H}), 7.05(\mathrm{~d}, J=8.6 \mathrm{~Hz}, 2 \mathrm{H}), 5.21(\mathrm{~d}, J=6.4 \mathrm{~Hz}$, $1 \mathrm{H}$ ), 4.89 (d, $J=6.4 \mathrm{~Hz}, 1 \mathrm{H}$ ), 4.65 (dd, $J=11.3,2.5 \mathrm{~Hz}, 1 \mathrm{H}$ ), 4.20 (ddt, $J=11.4,5.0,1.4 \mathrm{~Hz}, 1 \mathrm{H}$ ), 3.87 (td, $J=11.9,2.5 \mathrm{~Hz}, 1 \mathrm{H}$ ), 2.07 (dddd, $J=13.5,12.3,11.3,4.9 \mathrm{~Hz}, 1 \mathrm{H}$ ), $1.75-1.68(\mathrm{~m}, 1 \mathrm{H}), 1.35$ (s, $9 \mathrm{H})$.

${ }^{13} \mathrm{C} \mathrm{NMR}\left(126 \mathrm{MHz}, \mathrm{CDCl}_{3}\right) \delta 177.2(\mathrm{C}), 150.7(\mathrm{C}), 139.0(\mathrm{C}), 126.9(\mathrm{CH}), 121.6(\mathrm{CH}), 94.3\left(\mathrm{CH}_{2}\right)$, $78.3(\mathrm{CH}), 67.0\left(\mathrm{CH}_{2}\right), 39.2(\mathrm{C}), 34.2\left(\mathrm{CH}_{2}\right), 27.3\left(\mathrm{CH}_{3}\right)$.

ESI-HRMS: calculated for $\mathrm{C}_{15} \mathrm{H}_{20} \mathrm{O}_{4} \mathrm{Na}^{+}\left([\mathrm{M}+\mathrm{Na}]^{+}\right): 287.1254$, found: 287.1252 .

$\left[\alpha_{D}^{25}\right]=+35.3\left(c=0.221, \mathrm{CHCl}_{3}\right)$.

HPLC (Chiralpak IB-3 column, Heptane/ $i-\mathrm{PrOH} 98: 2,1 \mathrm{~mL} / \mathrm{min}, 25^{\circ} \mathrm{C}, 220 \mathrm{~nm}$ ): $\mathrm{t}_{\mathrm{R}}=4.9 \mathrm{~min}$ (minor), 5.4 min (major).

Racemate synthesis: Following General Procedure $D$ (condition A).

\section{(R)-S-(4-(1,3-dioxan-4-yl)phenyl) 2,2-dimethylpropanethioate (3e)}

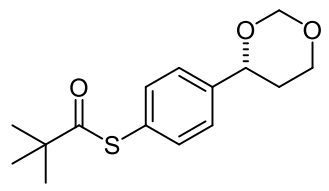

Prepared following General Procedure $C$, from $S$-(4-vinylphenyl) 2,2dimethylpropanethioate (1e, $54 \mu \mathrm{L}, 0.25 \mathrm{mmol}$ ), paraformaldehyde 2a (18.8 $\mathrm{mg}, 0.625 \mathrm{mmol}, 2.5$ equiv.), with catalyst $(S, S)-4 \mathrm{e}(12.4 \mathrm{mg}, 62.5 \mu \mathrm{mol}, 2.5$ mol\%), in dry cyclohexane ( $5 \mathrm{~mL}, 0.05 \mathrm{M}$ ) for $72 \mathrm{~h}$. Purification by flash column chromatography (eluent: $n$-pentane/MTBE $100: 0 \rightarrow 85: 15)$ to give $3 e$ as a white solid $(61.7 \mathrm{mg}, 88 \%$, er $=95: 5)$. 
${ }^{1} \mathrm{H}$ NMR $\left(501 \mathrm{MHz}, \mathrm{CDCl}_{3}\right) \delta 7.41(\mathrm{~d}, J=8.4 \mathrm{~Hz}, 2 \mathrm{H}), 7.38(\mathrm{~d}, J=8.7 \mathrm{~Hz}, 2 \mathrm{H}), 5.22(\mathrm{~d}, J=6.3 \mathrm{~Hz}$, $1 \mathrm{H}$ ), $4.89(\mathrm{~d}, J=6.4 \mathrm{~Hz}, 1 \mathrm{H}), 4.67$ (dd, $J=11.3,2.6 \mathrm{~Hz}, 1 \mathrm{H}), 4.20$ (dd, $J=11.5,4.8 \mathrm{~Hz}, 1 \mathrm{H}$ ), 3.88 (td, $J=11.9,2.5 \mathrm{~Hz}, 1 \mathrm{H}$ ), 2.06 (dddd, $J=13.4,12.1,11.2,4.8 \mathrm{~Hz}, 1 \mathrm{H}), 1.77-1.70(\mathrm{~m}, 1 \mathrm{H}), 1.32(\mathrm{~s}, 9 \mathrm{H})$.

${ }^{13} \mathrm{C}$ NMR $\left(126 \mathrm{MHz}, \mathrm{CDCl}_{3}\right) \delta 204.8(\mathrm{C}), 142.7(\mathrm{C}), 135.1(\mathrm{CH}), 127.5(\mathrm{C}), 126.5(\mathrm{CH}), 94.3\left(\mathrm{CH}_{2}\right)$, $78.3(\mathrm{CH}), 67.0\left(\mathrm{CH}_{2}\right), 47.1(\mathrm{C}), 34.1\left(\mathrm{CH}_{2}\right), 27.6\left(\mathrm{CH}_{3}\right)$.

ESI-HRMS: calculated for $\mathrm{C}_{15} \mathrm{H}_{20} \mathrm{O}_{3} \mathrm{SNa}^{+}\left([\mathrm{M}+\mathrm{Na}]^{+}\right)$: 303.1025 , found: 303.1024 .

$\left[\alpha_{D}^{25}\right]=+42.1\left(c=0.826, \mathrm{CHCl}_{3}\right)$.

HPLC (Chiralcel OJ-3 column, Heptane/ $i-\mathrm{PrOH} 95: 5,1 \mathrm{~mL} / \mathrm{min}, 25^{\circ} \mathrm{C}, 220 \mathrm{~nm}$ ): $\mathrm{t}_{\mathrm{R}}=12.4 \mathrm{~min}$ (minor), $21.2 \mathrm{~min}$ (major).

Racemate synthesis: Following General Procedure D (condition A).

\section{(R)-4-(4-fluorophenyl)-1,3-dioxane (3f)}

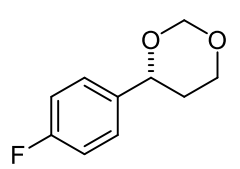

Prepared following General Procedure $C$, from 4-fluorostyrene (1f, $30 \mu \mathrm{L}, 0.25$ $\mathrm{mmol})$, paraformaldehyde $2 \mathrm{a}(18.8 \mathrm{mg}, 0.625 \mathrm{mmol}, 2.5$ equiv.), with catalyst $(S, S)$ 4d $(11.2 \mathrm{mg}, 62.5 \mu \mathrm{mol}, 2.5 \mathrm{~mol} \%)$, in dry cyclohexane $(5 \mathrm{~mL}, 0.05 \mathrm{M})$ for $96 \mathrm{~h}$. Purification by flash column chromatography (eluent: $n$-pentane/MTBE 100:0 $\rightarrow$ 95:5) to give $\mathbf{3 f}$ as a yellow solid (37.4 $\mathrm{mg}, 82 \%$, er $=94.5: 5.5)$.

${ }^{1} \mathrm{H}$ NMR $\left(501 \mathrm{MHz}, \mathrm{CDCl}_{3}\right) \delta 7.35$ (dd, $\left.J=8.6,5.5 \mathrm{~Hz}, 2 \mathrm{H}\right), 7.05(\mathrm{t}, J=8.7 \mathrm{~Hz}, 2 \mathrm{H}), 5.21$ (d, $J=6.3$ $\mathrm{Hz}, 1 \mathrm{H}), 4.89(\mathrm{~d}, J=6.4 \mathrm{~Hz}, 1 \mathrm{H}), 4.63(\mathrm{dd}, J=11.4,2.5 \mathrm{~Hz}, 1 \mathrm{H}), 4.21(\mathrm{dd}, J=11.6,4.7 \mathrm{~Hz}, 1 \mathrm{H}), 3.87$ (td, $J=11.9,2.5 \mathrm{~Hz}, 1 \mathrm{H}), 1.74-1.67(\mathrm{~m}, 1 \mathrm{H})$.

${ }^{19} \mathrm{~F} \mathrm{NMR}\left(471 \mathrm{MHz}, \mathrm{CDCl}_{3}\right) \delta-114.62(\mathrm{~s}, 1 \mathrm{~F})$.

${ }^{13} \mathrm{C}$ NMR $\left(126 \mathrm{MHz}, \mathrm{CDCl}_{3}\right) \delta 162.5(\mathrm{~d}, J=245.8 \mathrm{~Hz}, \mathrm{C}), 137.4(\mathrm{~d}, J=3.2 \mathrm{~Hz}, \mathrm{C}), 127.6(\mathrm{~d}, J=8.1 \mathrm{~Hz}$, $\mathrm{CH}), 115.5(\mathrm{~d}, J=21.3 \mathrm{~Hz}, \mathrm{CH}), 94.3\left(\mathrm{CH}_{2}\right), 78.2(\mathrm{CH}), 67.0\left(\mathrm{CH}_{2}\right), 34.1\left(\mathrm{CH}_{2}\right)$. Spectroscopic data was consistent with the values reported in the literature. ${ }^{16}$

ESI-HRMS: calculated for $\mathrm{C}_{10} \mathrm{H}_{11} \mathrm{FO}_{2} \mathrm{Na}^{+}\left([\mathrm{M}+\mathrm{Na}]^{+}\right)$: 205.0635, found: 205.0637 .

$\left[\alpha_{D}^{25}\right]=+34.1\left(c=0.369, \mathrm{CHCl}_{3}\right)$.

HPLC (Chiralpak IC-3 column, Heptane/i-PrOH 98:2, $1 \mathrm{~mL} / \mathrm{min}, 25^{\circ} \mathrm{C}, 220 \mathrm{~nm}$ ): $\mathrm{t}_{\mathrm{R}}=4.5 \mathrm{~min}$ (minor), 4.9 min (major).

Racemate synthesis: Following General Procedure D (condition B).

\section{(R)-4-(4-chlorophenyl)-1,3-dioxane (3g)}

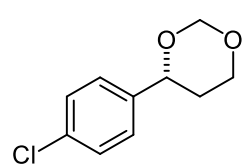

Prepared following General Procedure $C$, from 4-chlorostyrene $(\mathbf{1 g}, 30 \mu \mathrm{L}, 0.25$ $\mathrm{mmol}$ ), paraformaldehyde $2 \mathrm{a}$ (18.8 $\mathrm{mg}, 0.625 \mathrm{mmol}, 2.5$ equiv.), with catalyst $(S, S)$ 4 e $(12.4 \mathrm{mg}, 62.5 \mu \mathrm{mol}, 2.5 \mathrm{~mol} \%)$, in dry cyclohexane $(1.25 \mathrm{~mL}, 0.2 \mathrm{M})$ for $96 \mathrm{~h}$. Purification by flash column chromatography (eluent: $n$-pentane/MTBE 100:0 $\rightarrow$ 95:5) to give $\mathbf{3 g}$ as a colorless liquid $(43.5 \mathrm{mg}, 88 \%$, er $=95.5: 4.5)$.

${ }^{1} \mathrm{H}$ NMR $\left(501 \mathrm{MHz}, \mathrm{CDCl}_{3}\right) \delta 7.34(\mathrm{~d}, J=8.6 \mathrm{~Hz}, 2 \mathrm{H}), 7.30(\mathrm{~d}, J=8.6 \mathrm{~Hz}, 2 \mathrm{H}), 5.21(\mathrm{~d}, J=6.4 \mathrm{~Hz}$, $1 \mathrm{H}), 4.88(\mathrm{~d}, J=6.4 \mathrm{~Hz}, 1 \mathrm{H}$ ), $4.63(\mathrm{dd}, J=11.3,2.6 \mathrm{~Hz}, 1 \mathrm{H}$ ), 4.20 (ddt, $J=11.5,4.9,1.4 \mathrm{~Hz}, 1 \mathrm{H}$ ), 3.87 (td, $J=11.9,2.5 \mathrm{~Hz}, 1 \mathrm{H}$ ), 2.04 (dddd, $J=13.5,12.3,11.3,4.9 \mathrm{~Hz}, 1 \mathrm{H}), 1.74-1.67(\mathrm{~m}, 1 \mathrm{H})$.

${ }^{13} \mathrm{C}$ NMR $\left(126 \mathrm{MHz}, \mathrm{CDCl}_{3}\right) \delta 140.2(\mathrm{C}), 133.6(\mathrm{C}), 128.8(\mathrm{CH}), 127.2(\mathrm{CH}), 94.3\left(\mathrm{CH}_{2}\right), 78.1(\mathrm{CH})$, $66.9\left(\mathrm{CH}_{2}\right), 34.1\left(\mathrm{CH}_{2}\right)$. Spectroscopic data was consistent with the values reported in the literature. ${ }^{15}$ ESI-HRMS: calculated for $\mathrm{C}_{10} \mathrm{H}_{11} \mathrm{ClO}_{2} \mathrm{Na}^{+}\left([\mathrm{M}+\mathrm{Na}]^{+}\right): 221.0340$, found: 221.0342 .

$\left[\alpha_{D}^{25}\right]=+45.1\left(c=0.532, \mathrm{CHCl}_{3}\right)$.

HPLC (Chiralpak IC-3 column, Heptane/i-PrOH 97:3, $0.5 \mathrm{~mL} / \mathrm{min}, 25^{\circ} \mathrm{C}, 220 \mathrm{~nm}$ ): $\mathrm{t}_{\mathrm{R}}=8.0 \mathrm{~min}$ (minor), 8.7 min (major).

Racemate synthesis: Following General Procedure D (condition B). 


\section{(R)-4-(4-bromophenyl)-1,3-dioxane (3h)}

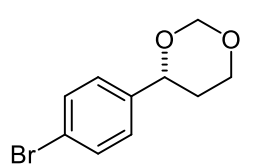

Prepared following General Procedure C, from 4-bromostyrene (1h, $33 \mu \mathrm{L}, 0.25$ $\mathrm{mmol}$ ), paraformaldehyde $2 \mathrm{a}$ ( $18.8 \mathrm{mg}, 0.625 \mathrm{mmol}, 2.5$ equiv.), with catalyst $(S, S)$ $4 \mathrm{e}(12.4 \mathrm{mg}, 62.5 \mu \mathrm{mol}, 2.5 \mathrm{~mol} \%)$, in dry cyclohexane $(1.25 \mathrm{~mL}, 0.2 \mathrm{M})$ for 5 days. Purification by flash column chromatography (eluent: $n$-pentane/MTBE 100:0 $\rightarrow$ 95:5) to give $3 \mathrm{~h}$ as a white solid (45.3 $\mathrm{mg}, 74 \%$, er $=95.5: 4.5)$.

${ }^{1} \mathrm{H}$ NMR $\left(501 \mathrm{MHz}, \mathrm{CDCl}_{3}\right) \delta 7.49(\mathrm{~d}, J=8.5 \mathrm{~Hz}, 2 \mathrm{H}), 7.25(\mathrm{~d}, J=8.4 \mathrm{~Hz}, 2 \mathrm{H}), 5.21(\mathrm{~d}, J=6.4 \mathrm{~Hz}$, $1 \mathrm{H}$ ), $4.88(\mathrm{~d}, J=6.4 \mathrm{~Hz}, 1 \mathrm{H}), 4.61$ (dd, $J=11.3,2.6 \mathrm{~Hz}, 1 \mathrm{H}), 4.20$ (dd, $J=11.4,4.8 \mathrm{~Hz}, 1 \mathrm{H}), 3.87$ (td, $J=11.9,2.4 \mathrm{~Hz}, 1 \mathrm{H}$ ), 2.03 (dddd, $J=13.5,12.3,11.3,4.9 \mathrm{~Hz}, 1 \mathrm{H}), 1.74-1.67(\mathrm{~m}, 1 \mathrm{H})$.

${ }^{13} \mathrm{C} \mathrm{NMR}\left(126 \mathrm{MHz}, \mathrm{CDCl}_{3}\right) \delta 140.7(\mathrm{C}), 131.7(\mathrm{CH}), 127.6(\mathrm{CH}), 121.7(\mathrm{C}), 94.3\left(\mathrm{CH}_{2}\right), 78.1(\mathrm{CH})$, $66.9\left(\mathrm{CH}_{2}\right), 34.1\left(\mathrm{CH}_{2}\right)$.

ESI-HRMS: calculated for $\mathrm{C}_{10} \mathrm{H}_{11} \mathrm{BrO}_{2} \mathrm{Na}^{+}\left([\mathrm{M}+\mathrm{Na}]^{+}\right)$: 264.9835 , found: 264.9836 .

$\left[\alpha_{D}^{25}\right]=+41.1\left(c=0.224, \mathrm{CHCl}_{3}\right)$.

HPLC (Chiralpak IC-3 column, Heptane/i-PrOH 97:3, $0.5 \mathrm{~mL} / \mathrm{min}, 25^{\circ} \mathrm{C}, 220 \mathrm{~nm}$ ): $\mathrm{t}_{\mathrm{R}}=8.3 \mathrm{~min}$ (minor), 9.2 min (major).

Racemate synthesis: Following General Procedure D (condition B).

\section{(R)-4-(3-fluorophenyl)-1,3-dioxane (3i)}

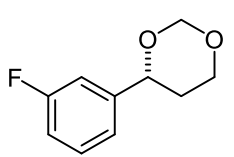

Prepared following General Procedure C, from 3-fluorostyrene $(\mathbf{1 i}, 30 \mu \mathrm{L}, 0.25$ $\mathrm{mmol}$ ), paraformaldehyde $2 \mathrm{a}$ ( $18.8 \mathrm{mg}, 0.625 \mathrm{mmol}, 2.5$ equiv.), with catalyst $(S, S)$ $4 \mathrm{e}(12.4 \mathrm{mg}, 62.5 \mu \mathrm{mol}, 2.5 \mathrm{~mol} \%)$, in dry cyclohexane $(1.25 \mathrm{~mL}, 0.2 \mathrm{M})$ for 5 days. Purification by flash column chromatography (eluent: $n$-pentane/MTBE 100:0 $\rightarrow$ 95:5)

to give $3 \mathbf{i}$ as a colorless liquid $(23.6 \mathrm{mg}, 52 \%$, er $=96.5: 3.5)$.

${ }^{1} \mathrm{H}$ NMR $\left(501 \mathrm{MHz}, \mathrm{CDCl}_{3}\right) \delta 7.32(\mathrm{td}, J=8.0,5.9 \mathrm{~Hz}, 1 \mathrm{H}), 7.15-7.07(\mathrm{~m}, 2 \mathrm{H}), 6.98$ (tdd, $J=8.4,2.6$, $1.0 \mathrm{~Hz}, 1 \mathrm{H}), 5.22(\mathrm{~d}, J=6.4 \mathrm{~Hz}, 1 \mathrm{H}), 4.89(\mathrm{~d}, J=6.4 \mathrm{~Hz}, 1 \mathrm{H}), 4.65(\mathrm{dd}, J=11.3,2.6 \mathrm{~Hz}, 1 \mathrm{H}), 4.20$ (ddt, $J=11.5,4.9,1.4 \mathrm{~Hz}, 1 \mathrm{H}$ ), 3.87 (td, $J=11.9,2.5 \mathrm{~Hz}, 1 \mathrm{H}$ ), 2.05 (dddd, $J=13.5,12.2,11.3,4.9 \mathrm{~Hz}$, $1 \mathrm{H}), 1.77-1.71(\mathrm{~m}, 1 \mathrm{H})$.

${ }^{19} \mathrm{~F} \mathrm{NMR}\left(471 \mathrm{MHz}, \mathrm{CDCl}_{3}\right) \delta-112.87(\mathrm{~s}, 1 \mathrm{~F})$.

${ }^{13} \mathrm{C}$ NMR (126 MHz, CDCl $) \delta 163.1$ (d, $\left.J=245.9 \mathrm{~Hz}, \mathrm{C}\right), 144.2$ (d, $\left.J=7.3 \mathrm{~Hz}, \mathrm{C}\right), 130.1$ (d, $J=8.0 \mathrm{~Hz}$, $\mathrm{CH}$ ), $121.3(\mathrm{~d}, J=2.8 \mathrm{~Hz}, \mathrm{CH}), 114.7(\mathrm{~d}, J=20.8 \mathrm{~Hz}, \mathrm{CH}), 112.9(\mathrm{~d}, J=22.4 \mathrm{~Hz}, \mathrm{CH}), 94.2\left(\mathrm{CH}_{2}\right)$, $78.0(\mathrm{~d}, J=2.1 \mathrm{~Hz}, \mathrm{CH}), 66.9\left(\mathrm{CH}_{2}\right), 34.0\left(\mathrm{CH}_{2}\right)$.

ESI-HRMS: calculated for $\mathrm{C}_{10} \mathrm{H}_{11} \mathrm{FO}_{2} \mathrm{Na}^{+}\left([\mathrm{M}+\mathrm{Na}]^{+}\right)$: 205.0635, found: 205.0637 .

$\left[\alpha_{D}^{25}\right]=+38.3\left(c=0.626, \mathrm{CHCl}_{3}\right)$.

HPLC (Chiralcel OJ-3 column, Heptane/ $\mathrm{i}-\mathrm{PrOH} 95: 5,1 \mathrm{~mL} / \mathrm{min}, 25^{\circ} \mathrm{C}, 220 \mathrm{~nm}$ ): $\mathrm{t}_{\mathrm{R}}=5.8 \mathrm{~min}$ (minor), 6.4 min (major).

Racemate synthesis: Following General Procedure D (condition C).

\section{(R)-4-(3-bromophenyl)-1,3-dioxane (3j)}

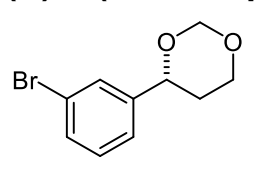

Prepared following General Procedure $C$, from 3-bromostyrene $(\mathbf{1 j}, 33 \mu \mathrm{L}, 0.25$ $\mathrm{mmol}$ ), paraformaldehyde $2 \mathrm{a}$ ( $18.8 \mathrm{mg}, 0.625 \mathrm{mmol}, 2.5$ equiv.), with catalyst $(S, S)$ 4b' (12.9 mg, $62.5 \mu \mathrm{mol}, 2.5 \mathrm{~mol} \%)$, in dry cyclohexane (1.25 mL, $0.2 \mathrm{M}$ ) for 5 days. Purification by flash column chromatography (eluent: $n$-pentane/MTBE 100:0 $\rightarrow$ 95:5) to give $\mathbf{3 j}$ as a colorless liquid (25.0 $\mathrm{mg}, 41 \%$, er $=96: 4)$.

${ }^{1} \mathrm{H}$ NMR $\left(501 \mathrm{MHz}, \mathrm{CDCl}_{3}\right) \delta 7.54(\mathrm{t}, J=1.9 \mathrm{~Hz}, 1 \mathrm{H}), 7.42$ (ddd, $\left.J=7.8,2.0,1.2 \mathrm{~Hz}, 1 \mathrm{H}\right), 7.29$ (d, $J=$ $7.9 \mathrm{~Hz}, 1 \mathrm{H}), 7.23(\mathrm{t}, J=7.8 \mathrm{~Hz}, 1 \mathrm{H}), 5.21(\mathrm{~d}, J=6.4 \mathrm{~Hz}, 1 \mathrm{H}), 4.88(\mathrm{~d}, J=6.4 \mathrm{~Hz}, 1 \mathrm{H}), 4.62(\mathrm{dd}, J=$ 11.3, $2.6 \mathrm{~Hz}, 1 \mathrm{H}$ ), 4.20 (dd, $J=11.5,4.8 \mathrm{~Hz}, 1 \mathrm{H}$ ), 3.87 (td, $J=11.9,2.4 \mathrm{~Hz}, 1 \mathrm{H}$ ), 2.04 (dddd, $J=13.4$, $12.1,11.3,4.8 \mathrm{~Hz}, 1 \mathrm{H}), 1.75-1.70(\mathrm{~m}, 1 \mathrm{H})$. 
${ }^{13} \mathrm{C}$ NMR (126 MHz, CDCl $)$ ) $143.9(\mathrm{C}), 131.0(\mathrm{CH}), 130.2(\mathrm{CH}), 129.0(\mathrm{CH}), 124.4(\mathrm{CH}), 122.8(\mathrm{C})$, $94.2\left(\mathrm{CH}_{2}\right), 78.0(\mathrm{CH}), 66.9\left(\mathrm{CH}_{2}\right), 34.1\left(\mathrm{CH}_{2}\right)$.

ESI-HRMS: calculated for $\mathrm{C}_{10} \mathrm{H}_{11} \mathrm{BrO}_{2} \mathrm{Na}^{+}\left([\mathrm{M}+\mathrm{Na}]^{+}\right)$: 264.9835 , found: 264.9835 .

$\left[\alpha_{D}^{25}\right]=+35.5\left(c=0.609, \mathrm{CHCl}_{3}\right)$.

HPLC (Chiralpak IC-3 column, Heptane/i-PrOH 97:3, $0.5 \mathrm{~mL} / \mathrm{min}, 25^{\circ} \mathrm{C}, 220 \mathrm{~nm}$ ): $\mathrm{tR}_{\mathrm{R}}=8.0 \mathrm{~min}$ (minor), 8.6 min (major).

Racemate synthesis: Following General Procedure D (condition C).

(R)-4-(m-tolyl)-1,3-dioxane (3k)

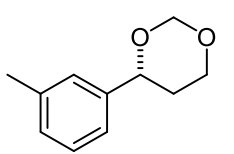

Prepared following General Procedure C, from 3-methylstyrene $(\mathbf{1 k}, 33 \mu \mathrm{L}, 0.25$ $\mathrm{mmol})$, paraformaldehyde $2 \mathrm{a}(18.8 \mathrm{mg}, 0.625 \mathrm{mmol}, 2.5$ equiv.), with catalyst $(S, S)$ 4e $(12.4 \mathrm{mg}, 62.5 \mu \mathrm{mol}, 2.5 \mathrm{~mol} \%)$, in dry cyclohexane $(5 \mathrm{~mL}, 0.05 \mathrm{M})$ for $72 \mathrm{~h}$. Purification by flash column chromatography (eluent: $n$-pentane/MTBE 100:0 $\rightarrow$ 95:5) to give $\mathbf{3 k}$ as a colorless liquid $(39.4 \mathrm{mg}, 88 \%$, er $=93: 7)$.

${ }^{1} \mathrm{H}$ NMR $\left(501 \mathrm{MHz}, \mathrm{CDCl}_{3}\right) \delta 7.25$ (t, $\left.J=7.6 \mathrm{~Hz}, 1 \mathrm{H}\right), 7.21$ (s, $\left.1 \mathrm{H}\right), 7.16(\mathrm{~d}, J=7.7 \mathrm{~Hz}, 1 \mathrm{H}), 7.11$ (d, $J$ $=7.6 \mathrm{~Hz}, 1 \mathrm{H}), 5.22(\mathrm{~d}, J=6.4 \mathrm{~Hz}, 1 \mathrm{H}), 4.90(\mathrm{~d}, J=6.3 \mathrm{~Hz}, 1 \mathrm{H}), 4.62(\mathrm{dd}, J=11.3,2.6 \mathrm{~Hz}, 1 \mathrm{H}), 4.21$ (dd, $J=11.4,4.9 \mathrm{~Hz}, 1 \mathrm{H}$ ), 3.88 (td, $J=11.9,2.5 \mathrm{~Hz}, 1 \mathrm{H}$ ), 2.36 (s, 3H), 2.11 (dddd, $J=13.5,12.3$, $11.3,4.8 \mathrm{~Hz}, 1 \mathrm{H}), 1.75-1.68(\mathrm{~m}, 1 \mathrm{H})$.

${ }^{13} \mathrm{C}$ NMR (126 MHz, $\left.\mathrm{CDCl}_{3}\right) \delta 141.5(\mathrm{C}), 138.3(\mathrm{C}), 128.7(\mathrm{CH}), 128.5(\mathrm{CH}), 126.6(\mathrm{CH}), 123.0(\mathrm{CH})$, $94.4\left(\mathrm{CH}_{2}\right), 79.0(\mathrm{CH}), 67.1\left(\mathrm{CH}_{2}\right), 34.1\left(\mathrm{CH}_{2}\right), 21.6\left(\mathrm{CH}_{3}\right)$.

ESI-HRMS: calculated for $\mathrm{C}_{11} \mathrm{H}_{14} \mathrm{O}_{2} \mathrm{Na}^{+}\left([\mathrm{M}+\mathrm{Na}]^{+}\right): 201.0886$, found: 201.0887 .

$\left[\alpha_{D}^{25}\right]=+40.5\left(c=0.430, \mathrm{CHCl}_{3}\right)$.

HPLC (Chiralpak IB-3 column, Heptane/i-PrOH 95:5, $1 \mathrm{~mL} / \mathrm{min}, 25^{\circ} \mathrm{C}, 220 \mathrm{~nm}$ ): $\mathrm{t}_{\mathrm{R}}=3.4 \mathrm{~min}$ (major), 4.3 min (minor).

Racemate synthesis: Following General Procedure $D$ (condition A).

\section{(R)-4-(3-methoxyphenyl)-1,3-dioxane (3I)}

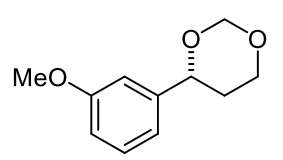

Prepared following General Procedure $C$, from 3-vinylanisole (1I, $34 \mu \mathrm{L}, 0.25$ mmol), paraformaldehyde 2a (18.8 $\mathrm{mg}, 0.625 \mathrm{mmol}, 2.5$ equiv.), with catalyst $(S, S)-4 e(12.4 \mathrm{mg}, 62.5 \mu \mathrm{mol}, 2.5 \mathrm{~mol} \%)$, in dry cyclohexane $(5 \mathrm{~mL}, 0.05 \mathrm{M})$ for 72

h. Purification by flash column chromatography (eluent: $n$-pentane/MTBE 100:0 $\rightarrow$ 90:10) to give $3 \mathbf{I}$ as a colorless oil (42.3 $\mathrm{mg}, 87 \%$, er $=93: 7)$.

${ }^{1} \mathrm{H}$ NMR $\left(501 \mathrm{MHz}, \mathrm{CDCl}_{3}\right) \delta 7.27(\mathrm{t}, J=7.8 \mathrm{~Hz}, 1 \mathrm{H}), 6.97-6.91(\mathrm{~m}, 2 \mathrm{H}), 6.84$ (ddd, $J=8.3,2.6,1.0$ $\mathrm{Hz}, 1 \mathrm{H}), 5.22(\mathrm{~d}, J=6.3 \mathrm{~Hz}, 1 \mathrm{H}), 4.90(\mathrm{~d}, J=6.3 \mathrm{~Hz}, 1 \mathrm{H}), 4.63(\mathrm{dd}, J=11.3,2.6 \mathrm{~Hz}, 1 \mathrm{H}), 4.20(\mathrm{dd}, J=$ $11.4,4.8 \mathrm{~Hz}, 1 \mathrm{H}$ ), $3.88(\mathrm{td}, J=11.9,2.5 \mathrm{~Hz}, 1 \mathrm{H}$ ), $3.82(\mathrm{~s}, 3 \mathrm{H}), 2.09$ (dddd, $J=13.5,12.2,11.3,4.9 \mathrm{~Hz}$, $1 \mathrm{H}), 1.75-1.70(\mathrm{~m}, 1 \mathrm{H})$.

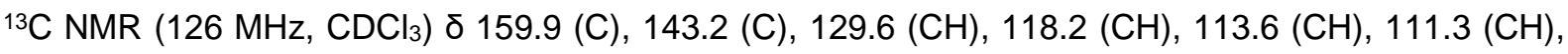
$94.3\left(\mathrm{CH}_{2}\right), 78.8(\mathrm{CH}), 67.1\left(\mathrm{CH}_{2}\right), 55.4\left(\mathrm{CH}_{3}\right), 34.1\left(\mathrm{CH}_{2}\right)$.

ESI-HRMS: calculated for $\mathrm{C}_{11} \mathrm{H}_{14} \mathrm{O}_{3} \mathrm{Na}^{+}\left([\mathrm{M}+\mathrm{Na}]^{+}\right): 217.0835$, found: 217.0837 .

$\left[\alpha_{D}^{25}\right]=+42.2\left(c=0.465, \mathrm{CHCl}_{3}\right)$.

HPLC (Chiralpak IB-3 column, Heptane/i-PrOH 95:5, $1 \mathrm{~mL} / \mathrm{min}, 25{ }^{\circ} \mathrm{C}, 220 \mathrm{~nm}$ ): $\mathrm{t}_{\mathrm{R}}=4.9 \mathrm{~min}$ (major), 6.4 min (minor).

Racemate synthesis: Following General Procedure D (condition A).

(R)-4-(3-bromo-4-methylphenyl)-1,3-dioxane (3m)

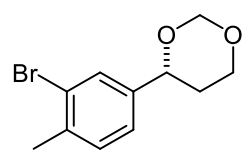

Prepared following General Procedure $C$, from 2-bromo-1-methyl-4-vinylbenzene ( $1 \mathrm{~m}, 37 \mu \mathrm{L}, 0.25 \mathrm{mmol}$ ), paraformaldehyde $2 \mathrm{a}$ (18.8 mg, $0.625 \mathrm{mmol}, 2.5$ equiv.), with catalyst $(S, S)-4 b(8.6 \mathrm{mg}, 62.5 \mu \mathrm{mol}, 2.5 \mathrm{~mol} \%)$, in dry cyclohexane $(1.25 \mathrm{~mL}$, 
$0.2 \mathrm{M})$ for 5 days. Purification by flash column chromatography (eluent: $n$-pentane/MTBE 100:0 $\rightarrow$ 95:5) to give $3 \mathrm{~m}$ as a colorless oil $(43.2 \mathrm{mg}, 67 \%$, er $=94: 6)$.

${ }^{1} \mathrm{H}$ NMR $\left(501 \mathrm{MHz}, \mathrm{CDCl}_{3}\right) \delta 7.56(\mathrm{~d}, J=1.6 \mathrm{~Hz}, 1 \mathrm{H}), 7.22(\mathrm{~d}, J=7.8 \mathrm{~Hz}, 1 \mathrm{H}), 7.19$ (dd, $J=7.8,1.6$ $\mathrm{Hz}, 1 \mathrm{H}$ ), 5.20 (d, J=6.4 Hz, 1H), $4.87(\mathrm{~d}, J=6.4 \mathrm{~Hz}, 1 \mathrm{H}), 4.59$ (dd, $J=11.3,2.6 \mathrm{~Hz}, 1 \mathrm{H}), 4.19$ (dd, $J=$ $11.5,4.9 \mathrm{~Hz}, 1 \mathrm{H}$ ), $3.86(\mathrm{td}, J=11.9,2.4 \mathrm{~Hz}, 1 \mathrm{H}), 2.38(\mathrm{~s}, 3 \mathrm{H}), 2.05$ (dddd, $J=13.5,12.2,11.3,4.9 \mathrm{~Hz}$, $1 \mathrm{H}), 1.73-1.68(\mathrm{~m}, 1 \mathrm{H})$.

${ }^{13} \mathrm{C} \mathrm{NMR}\left(126 \mathrm{MHz}, \mathrm{CDCl}_{3}\right) \delta 141.1(\mathrm{C}), 137.4(\mathrm{C}), 130.9(\mathrm{CH}), 129.9(\mathrm{CH}), 125.1(\mathrm{C}), 124.7(\mathrm{CH})$, $94.3\left(\mathrm{CH}_{2}\right), 77.8(\mathrm{CH}), 66.9\left(\mathrm{CH}_{2}\right), 34.0\left(\mathrm{CH}_{2}\right), 22.7\left(\mathrm{CH}_{3}\right)$.

ESI-HRMS: calculated for $\mathrm{C}_{11} \mathrm{H}_{13} \mathrm{BrO}_{2} \mathrm{Na}^{+}\left([\mathrm{M}+\mathrm{Na}]^{+}\right)$: 278.9991 , found: 278.9993 .

$\left[\alpha_{D}^{25}\right]=+32.0\left(c=0.651, \mathrm{CHCl}_{3}\right)$.

HPLC (Chiralcel OJ-3 column, Heptane/ $\mathrm{i}-\mathrm{PrOH} 95: 5,1 \mathrm{~mL} / \mathrm{min}, 25^{\circ} \mathrm{C}, 220 \mathrm{~nm}$ ): $\mathrm{t}_{\mathrm{R}}=6.2 \mathrm{~min}$ (minor), 7.6 min (major).

Racemate synthesis: Following General Procedure D (condition C).

\section{(R)-4-(naphthalen-2-yl)-1,3-dioxane (3n)}

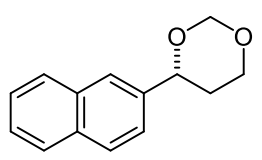

Prepared following General Procedure $C$, from 2-vinylnaphthalene (1n, $38.5 \mathrm{mg}$, $0.25 \mathrm{mmol}$ ), paraformaldehyde $2 \mathrm{a}$ ( $18.8 \mathrm{mg}, 0.625 \mathrm{mmol}, 2.5$ equiv.), with catalyst $(S, S)-4 e(12.4 \mathrm{mg}, 62.5 \mu \mathrm{mol}, 2.5 \mathrm{~mol} \%)$, in dry cyclohexane $(10 \mathrm{~mL}, 0.025 \mathrm{M})$ for 72 h. Purification by flash column chromatography (eluent: $n$-pentane/MTBE 100:0 $\rightarrow 90: 10)$ to give $3 \mathrm{n}$ as a white solid $(42.1 \mathrm{mg}, 79 \%$, er $=90.5: 9.5)$.

${ }^{1} \mathrm{H}$ NMR $\left(501 \mathrm{MHz}, \mathrm{CDCl}_{3}\right) \delta 7.88-7.81(\mathrm{~m}, 4 \mathrm{H}), 7.53-7.44(\mathrm{~m}, 3 \mathrm{H}), 5.29(\mathrm{~d}, J=6.4 \mathrm{~Hz}, 1 \mathrm{H}), 4.97$ (d, $J=6.4 \mathrm{~Hz}, 1 \mathrm{H}), 4.83(\mathrm{dd}, J=11.3,2.6 \mathrm{~Hz}, 1 \mathrm{H}), 4.25(\mathrm{dd}, J=11.4,4.8 \mathrm{~Hz}, 1 \mathrm{H}), 3.94(\mathrm{td}, J=11.8$, $2.5 \mathrm{~Hz}, 1 \mathrm{H}), 2.25-2.13(\mathrm{~m}, 1 \mathrm{H}), 1.85-1.79(\mathrm{~m}, 1 \mathrm{H})$.

${ }^{13} \mathrm{C}$ NMR (126 MHz, CDCl $)_{3} \delta 139.0(\mathrm{C}), 133.4(\mathrm{C}), 133.2(\mathrm{C}), 128.4(\mathrm{CH}), 128.2(\mathrm{CH}), 127.8(\mathrm{CH})$, $126.3(\mathrm{CH}), 126.1(\mathrm{CH}), 124.6(\mathrm{CH}), 124.0(\mathrm{CH}), 94.4\left(\mathrm{CH}_{2}\right), 78.9(\mathrm{CH}), 67.1\left(\mathrm{CH}_{2}\right), 34.2\left(\mathrm{CH}_{2}\right)$. Spectroscopic data was consistent with the values reported in the literature. ${ }^{15}$

ESI-HRMS: calculated for $\mathrm{C}_{14} \mathrm{H}_{14} \mathrm{O}_{2} \mathrm{Na}^{+}\left([\mathrm{M}+\mathrm{Na}]^{+}\right): 237.0886$, found: 237.0886 .

$\left[\alpha_{D}^{25}\right]=+21.0\left(c=0.591, \mathrm{CHCl}_{3}\right)$.

HPLC (Chiralpak IB-3 column, Heptane/ $i-\mathrm{PrOH} 80: 20,1 \mathrm{~mL} / \mathrm{min}, 25^{\circ} \mathrm{C}, 274 \mathrm{~nm}$ ): $\mathrm{tR}_{\mathrm{R}}=3.8 \mathrm{~min}$ (minor), 5.6 min (major).

Racemate synthesis: Following General Procedure $D$ (condition A).

\section{(R)-5-(1,3-dioxan-4-yl)-2,2-difluorobenzo[d][1,3]dioxole (3o)}

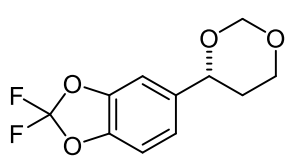

Prepared following General Procedure $C$, from 2,2-difluoro-5vinylbenzo[d][1,3]dioxole (10, $37 \mu \mathrm{L}, 0.25 \mathrm{mmol})$, paraformaldehyde $\mathbf{2 a}(18.8 \mathrm{mg}$, $0.625 \mathrm{mmol}, 2.5$ equiv.), with catalyst $(S, S)-4 \mathrm{~d}(11.2 \mathrm{mg}, 62.5 \mu \mathrm{mol}, 2.5 \mathrm{~mol} \%)$, in dry cyclohexane $(5 \mathrm{~mL}, 0.05 \mathrm{M})$ for $72 \mathrm{~h}$. Purification by flash column chromatography (eluent: $n$-pentane/MTBE 100:0 $\rightarrow$ 90:10) to give 30 as a colorless liquid $(35.0 \mathrm{mg}$, $57 \%$, er $=94.5: 5.5)$.

${ }^{1} \mathrm{H}$ NMR $\left(501 \mathrm{MHz}, \mathrm{CDCl}_{3}\right) \delta 7.14(\mathrm{~d}, J=1.6 \mathrm{~Hz}, 1 \mathrm{H}), 7.06$ (dd, $\left.J=8.2,1.6 \mathrm{~Hz}, 1 \mathrm{H}\right), 7.02(\mathrm{~d}, J=8.2$ $\mathrm{Hz}, 1 \mathrm{H}), 5.20(\mathrm{~d}, J=6.4 \mathrm{~Hz}, 1 \mathrm{H}), 4.88(\mathrm{~d}, J=6.4 \mathrm{~Hz}, 1 \mathrm{H}), 4.63(\mathrm{dd}, J=11.3,2.6 \mathrm{~Hz}, 1 \mathrm{H}), 4.20(\mathrm{dd}, J=$ $11.5,4.9 \mathrm{~Hz}, 1 \mathrm{H}$ ), 3.86 (td, $J=11.9,2.5 \mathrm{~Hz}, 1 \mathrm{H}$ ), 2.03 (dddd, $J=13.4,12.1,11.2,4.9 \mathrm{~Hz}, 1 \mathrm{H}$ ), $1.74-$ $1.68(\mathrm{~m}, 1 \mathrm{H})$.

${ }^{19} \mathrm{~F} \mathrm{NMR}\left(471 \mathrm{MHz}, \mathrm{CDCl}_{3}\right) \delta-50.03(\mathrm{~d}, J=3.5 \mathrm{~Hz}, 2 \mathrm{~F})$.

${ }^{13} \mathrm{C} \mathrm{NMR}\left(126 \mathrm{MHz}, \mathrm{CDCl}_{3}\right) \delta 144.1$ (C), 143.3 (C), 138.1 (C), 131.8 (t, $\left.J=254.9 \mathrm{~Hz}, \mathrm{C}\right), 121.0(\mathrm{CH})$, $109.3(\mathrm{CH}), 107.6(\mathrm{CH}), 94.3\left(\mathrm{CH}_{2}\right), 78.1(\mathrm{CH}), 66.9\left(\mathrm{CH}_{2}\right), 34.3\left(\mathrm{CH}_{2}\right)$.

El-HRMS: calculated for $\mathrm{C}_{11} \mathrm{H}_{10} \mathrm{~F}_{2} \mathrm{O}_{4}{ }^{+}\left([\mathrm{M}]^{+}\right): 244.0540$, found: 244.0542 .

$\left[\alpha_{D}^{25}\right]=+34.5\left(c=0.522, \mathrm{CHCl}_{3}\right)$. 
HPLC (Chiralcel OJ-3 column, Heptane/ $\mathrm{i}-\mathrm{PrOH} 95: 5,1 \mathrm{~mL} / \mathrm{min}, 25^{\circ} \mathrm{C}, 220 \mathrm{~nm}$ ): $\mathrm{t}_{\mathrm{R}}=5.2 \mathrm{~min}$ (major), 5.6 min (major).

Racemate synthesis: Following General Procedure D (condition A).

\section{(R)-4-(4-allylphenyl)-1,3-dioxane (3p)}

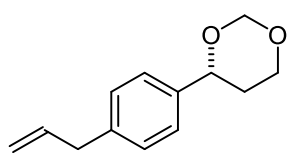

Prepared following General Procedure C, from 1-allyl-4-vinylbenzene $(1 \mathrm{p}, 40 \mu \mathrm{L}$, $0.25 \mathrm{mmol}$ ), paraformaldehyde 2a (18.8 $\mathrm{mg}, 0.625 \mathrm{mmol}, 2.5$ equiv.), with catalyst $(S, S)-4 b$ ( $8.6 \mathrm{mg}, 62.5 \mu \mathrm{mol}, 2.5 \mathrm{~mol} \%)$, in dry cyclohexane $(5 \mathrm{~mL}, 0.05$ M) for $72 \mathrm{~h}$. Purification by flash column chromatography (eluent: $n$ pentane/MTBE 100:0 $\rightarrow$ 95:5) to give $3 p$ as a colorless liquid $(45.3 \mathrm{mg}, 89 \%$, er $=91: 9$ ).

${ }^{1} \mathrm{H}$ NMR $\left(501 \mathrm{MHz}, \mathrm{CDCl}_{3}\right) \delta 7.31(\mathrm{~d}, J=8.1 \mathrm{~Hz}, 2 \mathrm{H}), 7.19(\mathrm{~d}, J=8.2 \mathrm{~Hz}, 2 \mathrm{H}), 5.96$ (ddt, $J=16.9$, $10.1,6.7 \mathrm{~Hz}, 1 \mathrm{H}), 5.21(\mathrm{~d}, J=6.3 \mathrm{~Hz}, 1 \mathrm{H}), 5.09(\mathrm{dq}, J=9.5,1.7 \mathrm{~Hz}, 1 \mathrm{H}), 5.06(\mathrm{p}, J=1.6 \mathrm{~Hz}, 1 \mathrm{H}), 4.90$ (d, $J=6.3 \mathrm{~Hz}, 1 \mathrm{H}$ ), 4.63 (dd, $J=11.3,2.5 \mathrm{~Hz}, 1 \mathrm{H}$ ), 4.21 (dd, $J=11.4,4.9 \mathrm{~Hz}, 1 \mathrm{H}$ ), 3.88 (td, $J=11.8$, $2.4 \mathrm{~Hz}, 1 \mathrm{H}$ ), 3.39 (d, $J=6.7 \mathrm{~Hz}, 2 \mathrm{H}$ ), 2.11 (dddd, $J=13.4,12.2,11.3,4.8 \mathrm{~Hz}, 1 \mathrm{H}), 1.73-1.68(\mathrm{~m}$, $1 \mathrm{H})$.

${ }^{13} \mathrm{C} \mathrm{NMR}\left(126 \mathrm{MHz}, \mathrm{CDCl}_{3}\right) \delta 139.8(\mathrm{C}), 139.4(\mathrm{C}), 137.5(\mathrm{CH}), 128.8(\mathrm{CH}), 126.1(\mathrm{CH}), 116.0\left(\mathrm{CH}_{2}\right)$, $94.4\left(\mathrm{CH}_{2}\right), 78.8(\mathrm{CH}), 67.1\left(\mathrm{CH}_{2}\right), 40.0\left(\mathrm{CH}_{2}\right), 34.0\left(\mathrm{CH}_{2}\right)$.

ESI-HRMS: calculated for $\mathrm{C}_{13} \mathrm{H}_{16} \mathrm{O}_{2} \mathrm{Na}^{+}\left([\mathrm{M}+\mathrm{Na}]^{+}\right): 227.1042$, found: 227.1041 .

$\left[\alpha_{D}^{25}\right]=+29.4\left(c=0.639, \mathrm{CHCl}_{3}\right)$.

HPLC (Chiralpak IC-3 column, Heptane/ $i$-PrOH 97:3, $0.5 \mathrm{~mL} / \mathrm{min}, 25^{\circ} \mathrm{C}, 220 \mathrm{~nm}$ ): $\mathrm{t}_{\mathrm{R}}=9.4 \mathrm{~min}$ (minor), 10.4 min (major).

Racemate synthesis: Following General Procedure D (condition A).

\section{(R)-4-phenyl-1,3-dioxane-5,5- $d_{2}\left(3 a\left(d_{2}\right)\right)$}

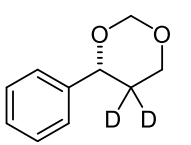

Prepared following General Procedure $C$, from styrene- $\beta, \beta-d_{2}\left(\mathbf{1 a}^{\prime}, 32 \mu \mathrm{L}, 0.25 \mathrm{mmol}\right)$, paraformaldehyde $2 \mathrm{a}$ ( $18.8 \mathrm{mg}, 0.625 \mathrm{mmol}, 2.5$ equiv.), with catalyst $(S, S)-4 \mathrm{c}(10.6 \mathrm{mg}$, $62.5 \mu \mathrm{mol}, 2.5 \mathrm{~mol} \%)$, in dry cyclohexane $(2.5 \mathrm{~mL}, 0.1 \mathrm{M})$ for $72 \mathrm{~h}$. Purification by flash column chromatography (eluent: $n$-pentane/MTBE 100:0 $\rightarrow$ 95:5) to give $3 \mathbf{a}\left(\boldsymbol{d}_{\mathbf{2}}\right)$ as a colorless liquid (30.4 mg, 73\%, er = 95:5).

${ }^{1} \mathrm{H}$ NMR $\left(501 \mathrm{MHz}, \mathrm{CDCl}_{3}\right) \delta 7.41-7.33(\mathrm{~m}, 4 \mathrm{H}), 7.33-7.26(\mathrm{~m}, 1 \mathrm{H}), 5.23(\mathrm{~d}, J=6.3 \mathrm{~Hz}, 1 \mathrm{H}), 4.90$ (d, $J=6.4 \mathrm{~Hz}, 1 \mathrm{H}), 4.65(\mathrm{~s}, 1 \mathrm{H}), 4.21(\mathrm{~d}, J=11.4 \mathrm{~Hz}, 1 \mathrm{H}), 3.88(\mathrm{dt}, J=11.5,1.9 \mathrm{~Hz}, 1 \mathrm{H})$.

${ }^{13} \mathrm{C} \mathrm{NMR}\left(126 \mathrm{MHz}, \mathrm{CDCl}_{3}\right) \delta 141.6(\mathrm{C}), 128.6(\mathrm{CH}), 128.0(\mathrm{CH}), 125.9(\mathrm{CH}), 94.3\left(\mathrm{CH}_{2}\right), 78.8(\mathrm{CH})$, $67.0\left(\mathrm{CH}_{2}\right)$. The carbon atom from the $\mathrm{CD}_{2}$ group was not visible.

El-HRMS: calculated for $\mathrm{C}_{10} \mathrm{H}_{10} \mathrm{D}_{2} \mathrm{O}_{2}{ }^{+}\left([\mathrm{M}]^{+}\right): 166.0957$, found: 166.0958 .

$\left[\alpha_{D}^{25}\right]=+27.5\left(c=0.793, \mathrm{CHCl}_{3}\right)$.

HPLC (Chiralpak IB-3 column, Heptane/ $i-\mathrm{PrOH} 95: 5,1 \mathrm{~mL} / \mathrm{min}, 25^{\circ} \mathrm{C}, 220 \mathrm{~nm}$ ): $\mathrm{t}_{\mathrm{R}}=3.6 \mathrm{~min}$ (major), 4.5 min (minor).

Racemate synthesis: Following General Procedure D (condition A).

\section{(R)-4-phenyl-1,3-dioxane-2,2,6,6- $d_{4}\left(3 \mathrm{a}\left(d_{4}\right)\right)$}

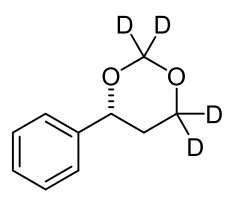

Prepared following General Procedure $C$, from styrene (1a, $29 \mu \mathrm{L}, 0.25 \mathrm{mmol}$ ), paraformaldehyde- $d_{2}$ 2a' (20.0 mg, $0.625 \mathrm{mmol}, 2.5$ equiv.), with catalyst $(S, S)-4 c$ (10.6 mg, $62.5 \mu \mathrm{mol}, 2.5 \mathrm{~mol} \%)$, in dry cyclohexane $(2.5 \mathrm{~mL}, 0.1 \mathrm{M})$ for $72 \mathrm{~h}$. Purification by flash column chromatography (eluent: $n$-pentane/MTBE 100:0 $\rightarrow$ 95:5) to give $3 \mathrm{a}\left(d_{4}\right)$ as a colorless liquid $(26.1 \mathrm{mg}, 62 \%$, er $=95: 5)$.

${ }^{1} \mathrm{H}$ NMR $\left(501 \mathrm{MHz}, \mathrm{CDCl}_{3}\right) \delta 7.40-7.34(\mathrm{~m}, 4 \mathrm{H}), 7.33-7.28(\mathrm{~m}, 1 \mathrm{H}), 4.66(\mathrm{dd}, J=11.3,2.6 \mathrm{~Hz}, 1 \mathrm{H})$, 2.09 (ddt, $J=13.2,11.2,1.8 \mathrm{~Hz}, 1 \mathrm{H}), 1.72(\mathrm{dd}, J=13.5,2.6 \mathrm{~Hz}, 1 \mathrm{H})$. 
${ }^{13} \mathrm{C}$ NMR (126 MHz, $\left.\mathrm{CDCl}_{3}\right) \delta 141.6(\mathrm{C}), 128.6(\mathrm{CH}), 128.0(\mathrm{CH}), 125.9(\mathrm{CH}), 78.8(\mathrm{CH}), 33.9\left(\mathrm{CH}_{2}\right)$. The carbon atoms from $\mathrm{CD}_{2}$ groups were not visible.

El-HRMS: calculated for $\mathrm{C}_{10} \mathrm{H}_{8} \mathrm{D}_{4} \mathrm{O}_{2}{ }^{+}\left([\mathrm{M}]^{+}\right)$: 168.1083 , found: 168.1084 .

$\left[\alpha_{D}^{25}\right]=+43.5\left(c=0.409, \mathrm{CHCl}_{3}\right)$.

HPLC (Chiralpak IB-3 column, Heptane/ $i-\mathrm{PrOH} 95: 5,1 \mathrm{~mL} / \mathrm{min}, 25^{\circ} \mathrm{C}, 220 \mathrm{~nm}$ ): $\mathrm{tR}_{\mathrm{R}}=3.6 \mathrm{~min}$ (major), 4.5 min (minor).

Racemate synthesis: Following General Procedure $D$ (condition A).

(R)-4-phenyl-1,3-dioxane-2,2,5,5,6,6- $d_{6}\left(3 \mathrm{a}\left(d_{6}\right)\right)$

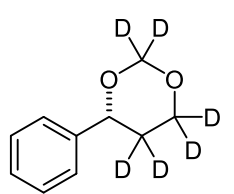

Prepared following General Procedure $C$, from styrene- $\beta, \beta-d_{2}$ (1)', $32 \mu \mathrm{L}, 0.25$ mmol), paraformaldehyde- $d_{2}$ 2a' (20.0 $\mathrm{mg}, 0.625 \mathrm{mmol}, 2.5$ equiv.), with catalyst $(S, S)-4 \mathrm{c}(10.6 \mathrm{mg}, 62.5 \mu \mathrm{mol}, 2.5 \mathrm{~mol} \%)$, in dry cyclohexane $(2.5 \mathrm{~mL}, 0.1 \mathrm{M})$ for $72 \mathrm{~h}$. Purification by flash column chromatography (eluent: $n$-pentane/MTBE 100:0 $\rightarrow$ 95:5) to give $3 \mathrm{a}\left(d_{6}\right)$ as a colorless liquid $(23.8 \mathrm{mg}, 56 \%$, er $=95: 5)$.

${ }^{1} \mathrm{H}$ NMR $\left(501 \mathrm{MHz}, \mathrm{CDCl}_{3}\right) \delta 7.40-7.34(\mathrm{~m}, 4 \mathrm{H}), 7.32-7.28(\mathrm{~m}, 1 \mathrm{H}), 4.65(\mathrm{~s}, 1 \mathrm{H})$.

${ }^{13} \mathrm{C} \mathrm{NMR}\left(126 \mathrm{MHz}, \mathrm{CDCl}_{3}\right) \delta 141.6(\mathrm{C}), 128.6(\mathrm{CH}), 128.0(\mathrm{CH}), 125.9(\mathrm{CH}), 78.7(\mathrm{CH})$. The carbon atoms from $C D_{2}$ groups were not visible.

ESI-HRMS: calculated for $\mathrm{C}_{10} \mathrm{H}_{6} \mathrm{D}_{6} \mathrm{O}_{2} \mathrm{Na}^{+}\left([\mathrm{M}+\mathrm{Na}]^{+}\right): 193.1106$, found: 193.1110 .

$\left[\alpha_{D}^{25}\right]=+44.2\left(c=0.371, \mathrm{CHCl}_{3}\right)$.

HPLC (Chiralpak IB-3 column, Heptane/ $i-\mathrm{PrOH} 95: 5,1 \mathrm{~mL} / \mathrm{min}, 25^{\circ} \mathrm{C}, 220 \mathrm{~nm}$ ): $\mathrm{t}_{\mathrm{R}}=3.6 \mathrm{~min}$ (major), 4.5 min (minor).

Racemate synthesis: Following General Procedure D (condition A).

\section{General Procedure D: Synthesis of racemates}

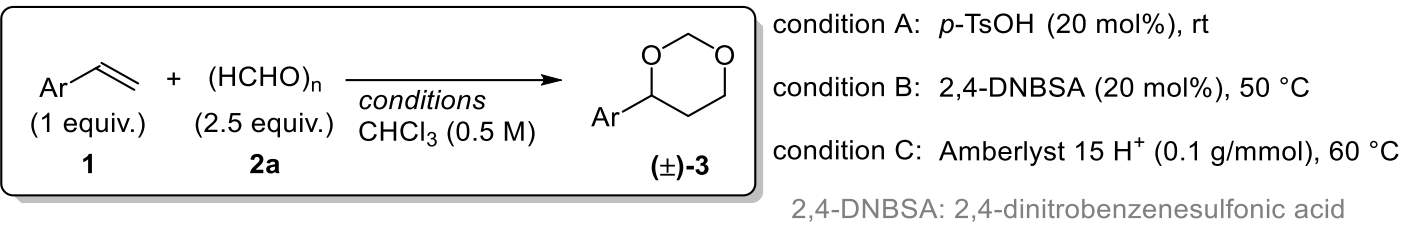

An oven-dried glass vial was charged with paraformaldehyde 2a (18.8 mg, $0.625 \mathrm{mmol}, 2.5$ equiv.), and with catalyst:

-either 20 mol\% p-toluenesulfonic acid (condition $A$ ),

-or $20 \mathrm{~mol} \%$ 2,4-dinitrobenzensulfonic acid hydrate (condition $B$ ),

-or $25 \mathrm{mg}$ Amberlyst $15 \mathrm{H}^{+}$(condition $\mathrm{C}$ ).

Then to it was added dry chloroform $(0.5 \mathrm{~mL})$, and olefin $1(0.25 \mathrm{mmol}, 1$ equiv.). The mixture was vigorously stirred, either at $\mathrm{rt}$ (condition $A$ ) or at $60^{\circ} \mathrm{C}$ (conditions $B-C$ ) for 24-72 $\mathrm{h}$. The reaction was stopped by adding distilled water $(10 \mathrm{~mL})$, followed by extraction with MTBE $(3 \times 20 \mathrm{~mL})$. The combined organic layers were washed with brine $(1 \times 10 \mathrm{~mL})$, then dried over anhydrous $\mathrm{Na}_{2} \mathrm{SO}_{4}$, filtered and concentrated under reduced pressure. Purification by flash column chromatography on silica gel ( $n$-pentane/MTBE mixtures) afforded the corresponding racemic 1,3-dioxane product 3. 


\section{1,3-Dioxane Ring Opening: Preparation of 1,3-Diol 5}

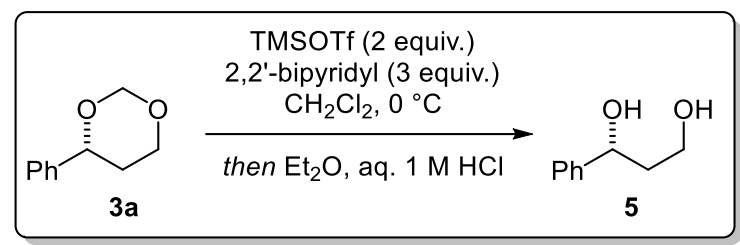

(Adapted from a reported procedure ${ }^{17}$ ): An oven-dried RB flask was charged with 3a (164 mg, $1 \mathrm{mmol}$, er = 95.5:4.5), dry $\mathrm{CH}_{2} \mathrm{Cl}_{2}(2 \mathrm{~mL})$ and 2,2'-bipyridyl (469 mg, $3 \mathrm{mmol}, 3$ equiv.). After cooling the mixture at $0{ }^{\circ} \mathrm{C}$, TMSOTf $(0.36 \mathrm{~mL}, 2 \mathrm{mmol}, 2$ equiv. $)$ was added dropwise followed by further stirring at $0^{\circ} \mathrm{C}$ for $2 \mathrm{~h}$. After checking full conversion of $3 a$ by TLC, Et $2 \mathrm{O}(10 \mathrm{~mL})$ and aq. $1 \mathrm{M} \mathrm{HCl}(10 \mathrm{~mL}, 10$ mmol, 10 equiv.) were added in one portion at $0{ }^{\circ} \mathrm{C}$ and the reaction mixture was stirred for $1.5 \mathrm{~h}$. The mixture was then extracted with EtOAc $(3 \times 30 \mathrm{~mL})$ and the combined organic phase was washed with satd. aq. $\mathrm{NaHCO}_{3}(1 \times 30 \mathrm{~mL})$, dried over anhydrous $\mathrm{Na}_{2} \mathrm{SO}_{4}$, filtered and concentrated under reduced pressure. Purification by flash column chromatography on silica gel ( $i$-hexane/EtOAc 90:10 $\rightarrow$ 60:40) afforded the corresponding 1,3-diol 5 as a colorless oil (134 $\mathrm{mg}, 88 \%$, er $=95: 5)$.

${ }^{1} \mathrm{H}$ NMR $\left(501 \mathrm{MHz}, \mathrm{CDCl}_{3}\right) \delta 7.39-7.33(\mathrm{~m}, 4 \mathrm{H}), 7.32-7.25(\mathrm{~m}, 1 \mathrm{H}), 4.96(\mathrm{dd}, J=8.8,3.7 \mathrm{~Hz}, 1 \mathrm{H})$, $3.89-3.83(\mathrm{~m}, 2 \mathrm{H}), 2.08-1.89(\mathrm{~m}, 2 \mathrm{H})$. Spectroscopic data was consistent with the values reported in the literature. ${ }^{18}$

HPLC (Chiralpak IC-3 column, Heptane/i-PrOH 95:5, $1 \mathrm{~mL} / \mathrm{min}, 25^{\circ} \mathrm{C}, 220 \mathrm{~nm}$ ): $\mathrm{t}_{\mathrm{R}}=16.3 \mathrm{~min}$ (minor), 22.8 min (major). 


\section{Current Limitations}

Several substrates proved to be challenging, since they are at the edges of the reactivity: some of them are either too reactive, whereas others display very low reactivity. Other substrates present moderate to good reactivity, but the enantioselectivity observed with the tested catalysts was not satisfactory. In any case, these substrates require further studies and optimization.

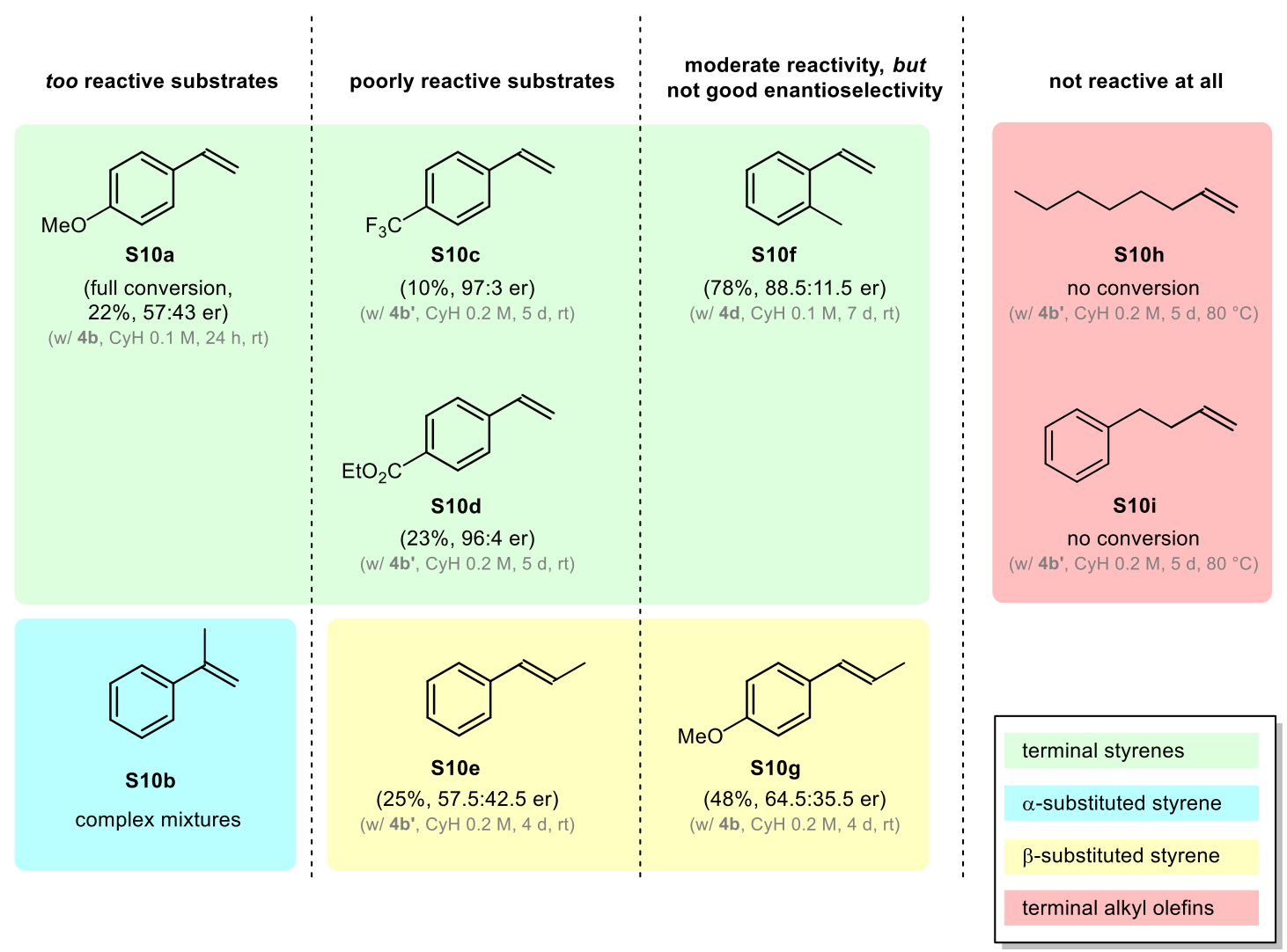

Figure S-1. Current substrate limitations of the ilDP-catalyzed Prins reaction.

\section{Terminal Styrenes}

Terminal styrenes containing strong EDG or EWG on the aromatic ring remained a challenge, although in opposite ways.

- 4-Vinylanisole (S10a) was fully consumed when either of the catalysts (4b, 4c, 4d, 4e) were used, forming complex mixtures from which the corresponding 1,3-dioxane was obtained only in moderate yields (e.g., with catalyst $\mathbf{4 b}$, in $\mathrm{CyH}, 0.1 \mathrm{M}$ for $24 \mathrm{~h}$ : full conversion, $22 \%$ yield, 57:43 er).

- 4-Trifluoromethylstyrene $(\mathbf{S 1 0})$ and ethyl 4-vinylbenzoate $(\mathbf{S} 10 \mathrm{~d})$ require the more acidic catalyst $\mathbf{4 b}$ ' $(2.5 \mathrm{~mol} \%)$ to show some conversion. After 5 days at rt (in $\mathrm{CyH}, 0.2 \mathrm{M}$ ), these olefins generate the corresponding 1,3-dioxanes in low yields, but with excellent enantioselectivity: $10 \%$ yield ( $97: 3$ er) and $23 \%$ yield ( $96: 4$ er), respectively.

- 2-Methylstyrene (S10f) reacts with paraformaldehyde in the presence of $2.5 \mathrm{~mol} \%$ of catalyst 4d (in $\mathrm{CyH}, 0.1 \mathrm{M}$ ), to produce $78 \%$ of the corresponding 1,3-dioxane after 7 days, but with moderate enantioselectivity (88.5:11.5 er).

\section{$\alpha-/ \beta$-SUBSTITUTED STYRENES}

- $\quad \alpha$-Methylstyrene (S10b) proved to be highly reactive with all the tested iDP catalysts (2.5 mol\%), giving always high conversion (>90\%) after 24-48 h (in $\mathrm{CyH}, 0.1 \mathrm{M}$, at rt); however, 
complex mixtures were obtained and the corresponding 1,3-dioxane could not be detected by ${ }^{1} \mathrm{H}$ NMR. Neither lower catalyst loadings $(1 \mathrm{~mol} \%)$ nor lower temperatures $\left(0^{\circ} \mathrm{C}\right)$ were helpful: the reaction showed then lower conversion, but still forming complex mixtures.

- trans- $\beta$-Methylstyrene (S10e) proved not reactive with the standard iDP catalysts (no 1,3dioxane detected by ${ }^{~} \mathrm{H}$ NMR after 5 days at $\mathrm{rt}$ ). The more acidic ilDP $\mathbf{4} \mathbf{b}$ ' as catalyst provided $25 \%$ of trans-5-methyl-4-phenyl-1,3-dioxane, although with low enantioselectivity (er = 57.5:42.5).

- In an attempt to improve the reactivity of internal olefins, trans-anethole ( $\mathbf{S 1 0 g})$ was tested as substrate. Gratifyingly, utilizing catalyst $\mathbf{4 b}$ resulted in $48 \%$ yield of the corresponding trans1,3-dioxane after 4 days at rt $(\mathrm{CyH}, 0.2 \mathrm{M})$, although only with modest enantioselectivity (er = 64.5:35.5).

- Interestingly, the ilDP-catalyzed reactions with the internal olefins (S10e or $\mathbf{S 1 0 g}$ ) displayed a high degree of diastereospecificity (trans-olefins produce trans-1,3-dioxanes). This goes in line with the outcome from the experiments using styrene- $\beta-d$ (section 6.2 of this Supponrting Information file).

\section{ALKYL OLEFINS}

Despite all the efforts devoted to obtain reactivity from alkyl olefins, such as 1-octene (S10h), none of the tested catalysts provided any substrate conversion, neither at rt nor at higher temperatures $\left(50{ }^{\circ} \mathrm{C}\right.$ or $80^{\circ} \mathrm{C}$ ). Considering that aromatic rings could act as a recognition element for the ilDP catalysts, 4phenyl-1-butene (S10i) was tested as substrate, but unfortunately no reactivity was observed.

\section{(R)-4-(4-methoxyphenyl)-1,3-dioxane (S11a)}

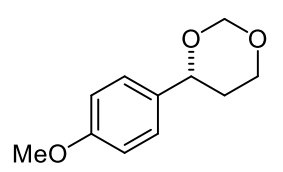

Prepared following General Procedure C, from 4-vinylanisole (S10a, $33 \mu \mathrm{L}, 0.25$ $\mathrm{mmol})$, paraformaldehyde $2 \mathrm{a}$ (18.8 $\mathrm{mg}, 0.625 \mathrm{mmol}, 2.5$ equiv.), with catalyst $(S, S)-4 b(8.6 \mathrm{mg}, 62.5 \mu \mathrm{mol}, 2.5 \mathrm{~mol} \%)$, in dry cyclohexane $(2.5 \mathrm{~mL}, 0.1 \mathrm{M})$ for 72 h. Purification by flash column chromatography (eluent: $n$-pentane/MTBE 100:0 $\rightarrow$ $80: 20)$ to give S11a as a white solid $(10.7 \mathrm{mg}, 22 \%$, er $=57: 43)$.

${ }^{1} \mathrm{H}$ NMR $\left(501 \mathrm{MHz}, \mathrm{CDCl}_{3}\right) \delta 7.30(\mathrm{~d}, J=8.6 \mathrm{~Hz}, 2 \mathrm{H}), 6.90(\mathrm{~d}, J=8.8 \mathrm{~Hz}, 2 \mathrm{H}), 5.20(\mathrm{~d}, J=6.3 \mathrm{~Hz}$, $1 \mathrm{H}$ ), $4.89(\mathrm{~d}, J=6.3 \mathrm{~Hz}, 1 \mathrm{H}$ ), 4.59 (dd, $J=11.2,2.5 \mathrm{~Hz}, 1 \mathrm{H}$ ), 4.20 (ddt, $J=11.5,4.9,1.4 \mathrm{~Hz}, 1 \mathrm{H}$ ), 3.87 (td, $J=11.8,2.5 \mathrm{~Hz}, 1 \mathrm{H}$ ), 3.81 (s, 3H), 2.11 (dddd, $J=13.5,12.3,11.3,4.9 \mathrm{~Hz}, 1 \mathrm{H}$ ), $1.71-1.66(\mathrm{~m}$, $1 \mathrm{H})$.

${ }^{13} \mathrm{C} \mathrm{NMR}\left(126 \mathrm{MHz}, \mathrm{CDCl}_{3}\right) \delta 159.4(\mathrm{C}), 133.8(\mathrm{C}), 127.3(\mathrm{CH}), 114.0(\mathrm{CH}), 94.4\left(\mathrm{CH}_{2}\right), 78.6(\mathrm{CH})$, $67.1\left(\mathrm{CH}_{2}\right), 55.4\left(\mathrm{CH}_{3}\right), 34.0\left(\mathrm{CH}_{2}\right)$. Spectroscopic data was consistent with the values reported in the literature. ${ }^{19}$

ESI-HRMS: calculated for $\mathrm{C}_{11} \mathrm{H}_{14} \mathrm{O}_{3} \mathrm{Na}^{+}\left([\mathrm{M}+\mathrm{Na}]^{+}\right): 217.0835$, found: 217.0836 .

HPLC (Chiralpak AD-3 column, Heptane/i-PrOH 97:3, $0.5 \mathrm{~mL} / \mathrm{min}, 25^{\circ} \mathrm{C}, 220 \mathrm{~nm}$ ): $\mathrm{t}_{\mathrm{R}}=11.2 \mathrm{~min}$ (major), 13.2 min (minor).

Racemate synthesis: Following General Procedure D (condition A).

\section{(R)-4-(4-(trifluoromethyl)phenyl)-1,3-dioxane (S11c)}

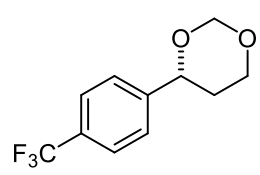

Prepared following General Procedure C, from 4-(trifluoromethyl)styrene (S10c, 37 $\mu \mathrm{L}, 0.25 \mathrm{mmol}$ ), paraformaldehyde $2 \mathrm{a}(18.8 \mathrm{mg}, 0.625 \mathrm{mmol}, 2.5$ equiv.), with catalyst $(S, S)-4 b^{\prime}$ (12.9 mg, $\left.62.5 \mu \mathrm{mol}, 2.5 \mathrm{~mol} \%\right)$, in dry cyclohexane $(1.25 \mathrm{~mL}, 0.2$ M) for 5 days. Purification by flash column chromatography (eluent: $n$ pentane/MTBE 100:0 $\rightarrow$ 95:5) to give S11c as a white solid (5.8 $\mathrm{mg}, 10 \%$, er $=97: 3$ ). 
${ }^{1} \mathrm{H}$ NMR $\left(501 \mathrm{MHz}, \mathrm{CDCl}_{3}\right) \delta 7.62(\mathrm{~d}, J=8.1 \mathrm{~Hz}, 2 \mathrm{H}), 7.49(\mathrm{~d}, J=8.1 \mathrm{~Hz}, 2 \mathrm{H}), 5.24(\mathrm{~d}, J=6.4 \mathrm{~Hz}$, $1 \mathrm{H}), 4.90(\mathrm{~d}, J=6.4 \mathrm{~Hz}, 1 \mathrm{H}), 4.72(\mathrm{dd}, J=11.3,2.6 \mathrm{~Hz}, 1 \mathrm{H}), 4.25-4.18(\mathrm{~m}, 1 \mathrm{H}), 3.89(\mathrm{td}, J=11.9$, $2.5 \mathrm{~Hz}, 1 \mathrm{H}$ ), 2.04 (dddd, $J=13.5,12.2,11.3,4.9 \mathrm{~Hz}, 1 \mathrm{H}), 1.78-1.73(\mathrm{~m}, 1 \mathrm{H})$.

${ }^{19} \mathrm{~F} \mathrm{NMR}\left(471 \mathrm{MHz}, \mathrm{CDCl}_{3}\right) \delta-62.54(\mathrm{~s}, 3 \mathrm{~F})$.

${ }^{13} \mathrm{C} \mathrm{NMR}\left(126 \mathrm{MHz}, \mathrm{CDCl}_{3}\right) \delta 145.6(\mathrm{C}), 130.1$ (q, $\left.J=32.5 \mathrm{~Hz}, \mathrm{C}\right), 126.1(\mathrm{CH}), 125.6(\mathrm{q}, J=3.8 \mathrm{~Hz}$, $\mathrm{CH}), 124.2(\mathrm{q}, J=271.8 \mathrm{~Hz}, \mathrm{C}), 94.2\left(\mathrm{CH}_{2}\right), 78.0(\mathrm{CH}), 66.9\left(\mathrm{CH}_{2}\right), 34.1\left(\mathrm{CH}_{2}\right)$.

ESI-HRMS: calculated for $\mathrm{C}_{11} \mathrm{H}_{11} \mathrm{~F}_{3} \mathrm{O}_{2} \mathrm{Na}^{+}\left([\mathrm{M}+\mathrm{Na}]^{+}\right)$: 255.0603 , found: 255.0602 .

$\left[\alpha_{D}^{25}\right]=+40.2\left(c=0.249, \mathrm{CHCl}_{3}\right)$.

HPLC (Kromasil Amycoat RP column, $\mathrm{CH}_{3} \mathrm{CN} / \mathrm{H}_{2} \mathrm{O} 50: 50,1 \mathrm{~mL} / \mathrm{min}, 25^{\circ} \mathrm{C}, 220 \mathrm{~nm}$ ): $\mathrm{t}_{\mathrm{R}}=7.7 \mathrm{~min}$ (major), 8.5 min (minor).

Racemate synthesis: Following General Procedure D (condition C).

\section{ethyl (R)-4-(1,3-dioxan-4-yl)benzoate (S11d)}

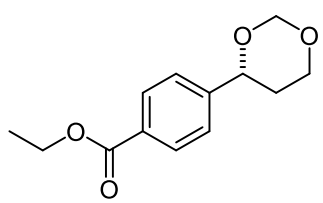

Prepared following General Procedure C, from ethyl 4-vinylbenzoate (S10d, 43 $\mu \mathrm{L}, 0.25 \mathrm{mmol})$, paraformaldehyde $2 \mathrm{a}(18.8 \mathrm{mg}, 0.625 \mathrm{mmol}, 2.5$ equiv.), with catalyst $(S, S)-4 b^{\prime}(12.9 \mathrm{mg}, 62.5 \mu \mathrm{mol}, 2.5 \mathrm{~mol} \%)$, in dry cyclohexane $(1.25$ $\mathrm{mL}, 0.2 \mathrm{M}$ ) for 5 days. Purification by flash column chromatography (eluent: $n$ pentane/MTBE 100:0 $\rightarrow$ 85:15) to give S11d as a white solid $(13.4 \mathrm{mg}, 23 \%$, er

$=96: 4)$.

${ }^{1} \mathrm{H}$ NMR $\left(501 \mathrm{MHz}, \mathrm{CDCl}_{3}\right) \delta 8.04(\mathrm{~d}, J=8.4 \mathrm{~Hz}, 2 \mathrm{H}), 7.44(\mathrm{~d}, J=8.2 \mathrm{~Hz}, 2 \mathrm{H}), 5.24(\mathrm{~d}, J=6.4 \mathrm{~Hz}$, $1 \mathrm{H}), 4.90(\mathrm{~d}, J=6.4 \mathrm{~Hz}, 1 \mathrm{H}), 4.71(\mathrm{dd}, J=11.3,2.6 \mathrm{~Hz}, 1 \mathrm{H}), 4.38(\mathrm{q}, J=7.1 \mathrm{~Hz}, 2 \mathrm{H}), 4.21$ (dd, $J=$ $11.5,4.8 \mathrm{~Hz}, 1 \mathrm{H}$ ), 3.89 (td, $J=11.9,2.5 \mathrm{~Hz}, 1 \mathrm{H}$ ), 2.04 (dddd, $J=13.4,12.2,11.3,4.8 \mathrm{~Hz}, 1 \mathrm{H}$ ), $1.78-$ $1.72(\mathrm{~m}, 1 \mathrm{H}), 1.39(\mathrm{t}, J=7.1 \mathrm{~Hz}, 3 \mathrm{H})$.

${ }^{13} \mathrm{C} \mathrm{NMR}\left(126 \mathrm{MHz}, \mathrm{CDCl}_{3}\right) \delta 166.5(\mathrm{C}), 146.5(\mathrm{C}), 130.0(\mathrm{C}), 129.9(\mathrm{CH}), 125.6(\mathrm{CH}), 94.2\left(\mathrm{CH}_{2}\right)$, $78.3(\mathrm{CH}), 66.9\left(\mathrm{CH}_{2}\right), 61.1\left(\mathrm{CH}_{2}\right), 34.1\left(\mathrm{CH}_{2}\right), 14.5\left(\mathrm{CH}_{3}\right)$.

ESI-HRMS: calculated for $\mathrm{C}_{13} \mathrm{H}_{16} \mathrm{O}_{4} \mathrm{Na}^{+}\left([\mathrm{M}+\mathrm{Na}]^{+}\right)$: 259.0941 , found: 259.0944 .

$\left[\alpha_{D}^{25}\right]=+28.5\left(c=0.548, \mathrm{CHCl}_{3}\right)$.

HPLC (Chiralpak AD-3 column, Heptane/ $i-\mathrm{PrOH} 95: 5,1 \mathrm{~mL} / \mathrm{min}, 25^{\circ} \mathrm{C}, 220 \mathrm{~nm}$ ): $\mathrm{t}_{\mathrm{R}}=7.1 \mathrm{~min}$ (major), 8.4 min (minor).

Racemate synthesis: Following General Procedure D (condition C).

\section{(R)-4-(o-tolyl)-1,3-dioxane (S11f)}

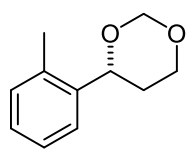

Prepared following General Procedure C, from 2-methylstyrene (S10f, $32 \mu \mathrm{L}, 0.25$ mmol), paraformaldehyde $2 \mathbf{2 a}(18.8 \mathrm{mg}, 0.625 \mathrm{mmol}, 2.5$ equiv.), with catalyst $(S, S)-\mathbf{4 d}$ $(11.2 \mathrm{mg}, 62.5 \mu \mathrm{mol}, 2.5 \mathrm{~mol} \%)$, in dry cyclohexane $(2.5 \mathrm{~mL}, 0.1 \mathrm{M})$ for 7 days. Purification by flash column chromatography (eluent: $n$-pentane/MTBE 100:0 $\rightarrow 95: 5$ ) to give $\mathbf{S 1 1 f}$ as a colorless liquid $(23.1 \mathrm{mg}, 78 \%$, er $=88.5: 11.5)$.

${ }^{1} \mathrm{H}$ NMR $\left(501 \mathrm{MHz}, \mathrm{CDCl}_{3}\right) \delta 7.50$ (dd, $\left.J=7.6,1.7 \mathrm{~Hz}, 1 \mathrm{H}\right), 7.25$ (t, $\left.J=8.4 \mathrm{~Hz}, 1 \mathrm{H}\right), 7.20$ (td, $J=7.4$, $1.5 \mathrm{~Hz}, 1 \mathrm{H}), 7.15(\mathrm{~d}, J=7.4 \mathrm{~Hz}, 1 \mathrm{H}), 5.24(\mathrm{~d}, J=6.3 \mathrm{~Hz}, 1 \mathrm{H}), 4.93(\mathrm{~d}, J=6.3 \mathrm{~Hz}, 1 \mathrm{H}), 4.83(\mathrm{dd}, J=$ 11.2, $2.4 \mathrm{~Hz}, 1 \mathrm{H}$ ), 4.22 (dd, $J=11.4,4.8 \mathrm{~Hz}, 1 \mathrm{H}$ ), 3.89 (td, $J=11.9,2.4 \mathrm{~Hz}, 1 \mathrm{H}$ ), 2.35 (s, 3H), 2.09 (dddd, $J=13.6,12.3,11.2,4.8 \mathrm{~Hz}, 1 \mathrm{H}), 1.73-1.66(\mathrm{~m}, 1 \mathrm{H})$.

${ }^{13} \mathrm{C} \mathrm{NMR}\left(126 \mathrm{MHz}, \mathrm{CDCl}_{3}\right) \delta 139.5(\mathrm{C}), 134.4(\mathrm{C}), 130.5(\mathrm{CH}), 127.7(\mathrm{CH}), 126.5(\mathrm{CH}), 125.9(\mathrm{CH})$, $94.5\left(\mathrm{CH}_{2}\right), 76.2(\mathrm{CH}), 67.2\left(\mathrm{CH}_{2}\right), 32.8\left(\mathrm{CH}_{2}\right), 19.1\left(\mathrm{CH}_{3}\right)$.

ESI-HRMS: calculated for $\mathrm{C}_{11} \mathrm{H}_{14} \mathrm{O}_{2} \mathrm{Na}^{+}\left([\mathrm{M}+\mathrm{Na}]^{+}\right): 201.0886$, found: 201.0888 .

$\left[\alpha_{D}^{25}\right]=+37.6\left(c=0.622, \mathrm{CHCl}_{3}\right)$.

HPLC (Chiralpak IC-3 column, Heptane/ $i-\mathrm{PrOH} 97: 3,0.5 \mathrm{~mL} / \mathrm{min}, 25^{\circ} \mathrm{C}, 220 \mathrm{~nm}$ ): $\mathrm{t}_{\mathrm{R}}=9.1 \mathrm{~min}$ (minor), 10.1 min (major).

Racemate synthesis: Following General Procedure D (condition B). 


\section{Mechanistic Studies}

The acid-catalyzed intermolecular Prins reaction (olefins + formaldehyde) is thought to proceed via a stepwise mechanism (as depicted in Scheme S-1), starting with a nucleophilic attack of the olefin to an activated molecule of formaldehyde $\mathbf{I} \mathbf{- 1}$ to form an intermediate $\mathbf{y}$-hydroxybenzyl cation $\mathbf{I - 2}$. The fate of this species explains the several products commonly obtained (unsaturated alcohols, 1,3-glycols and esters thereof, and 1,3-dioxanes, among others). For example, the formation of the 1,3-dioxane product 3 can be explained if the intermediate $\mathbf{~}-2$ further reacts with a second molecule of formaldehyde, forming a cationic hemiformal I-3 that readily undergoes cyclization.

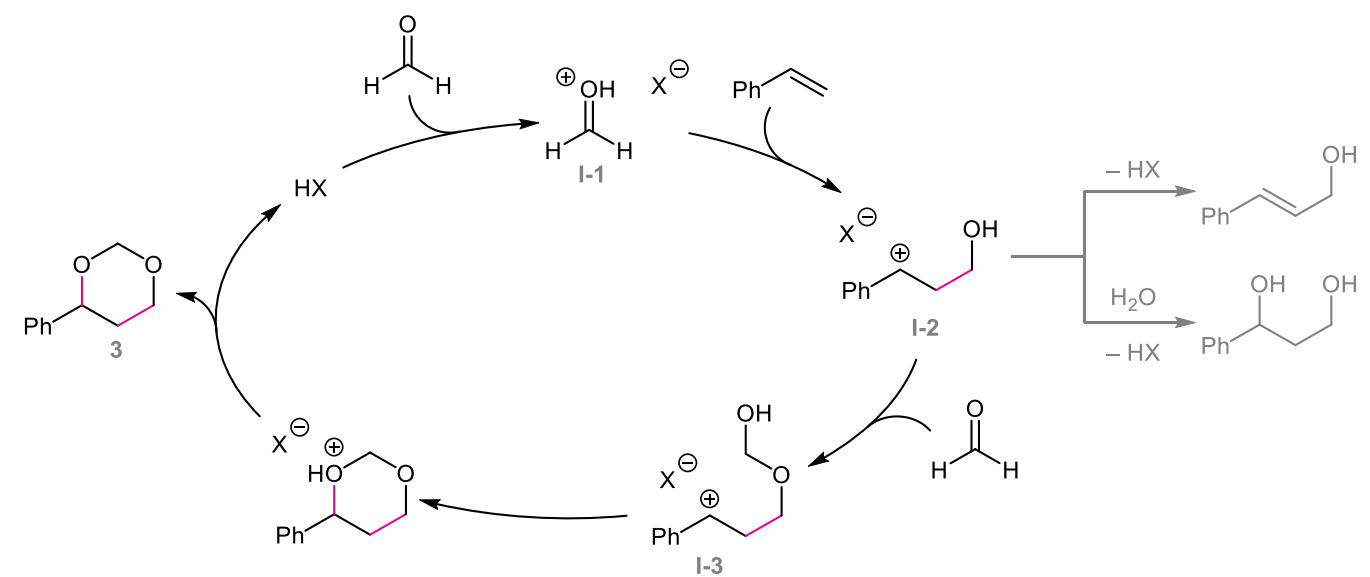

Scheme S-1. Proposed mechanistic cycle for an acid-catalyzed intermolecular Prins reaction via a stepwise pathway.

Now, considering that our ilDP-catalyzed Prins reaction proceeds with paraformaldehyde as $\mathrm{HCHO}$ source, the polymeric nature of this reagent should be taken into account when proposing a reaction mechanism. In order to do so, we wanted to gain a better understanding on how the two formaldehyde units get bonded to the olefin moiety.

The two HCHO units could come:

- either from different polymeric $\left(\mathrm{CH}_{2} \mathrm{O}\right)_{n}$ chains, or

- from the same polymeric $\left(\mathrm{CH}_{2} \mathrm{O}\right)_{n}$ chain:

- if so: do the two $\mathrm{HCHO}$ units react with the olefin at the same time or in different steps?

By addressing these issues, we could know if the operating mechanistic pathway is stepwise or rather a (pseudo)-concerted one (see Figure 3A, in the main text).

\subsection{Deuterium incorporation: $(\mathrm{HCHO})_{n}$ and $(\mathrm{DCDO})_{n}$}

To determine if the two formaldehyde units that are bonded to the olefin moiety during the Prins reaction could come from the same polymeric chain of paraformaldehyde, an experiment was designed, where styrene 1a reacts with mixtures of "protio"-paraformaldehyde $((\mathrm{HCHO}) n, 2 a)$ and deutero-paraformaldehyde $((D C D O) n, 2 a$ '), both commercially available.

Two possible scenarios were predicted:

- If the two formaldehyde units are bonded to the olefin in different steps, a statistical mixture of 1,3-dioxane products should be obtained, where the amount of ${ }^{1} \mathrm{H}$ (and also of ${ }^{2} \mathrm{H}$ ) on positions C-2 and C-6 would be different from each other.

- Contrarily, if both formaldehyde units were to come from the same paraformaldehyde chain, every produced molecule of 1,3-dioxane should contain the same isotope (H or $D)$ on both positions $\mathrm{C}-2$ and $\mathrm{C}-6$. This would be reflected in a C-2/C-6 ${ }^{1} \mathrm{H}$ content ratio close to 1 . 
The relative content of ${ }^{1} \mathrm{H}$ of positions $\mathrm{C}-2$ and $\mathrm{C}-6$ can be determined using ${ }^{1} \mathrm{H}$ NMR, since the integral values are proportional to the amount of ${ }^{1} \mathrm{H}$ atoms.

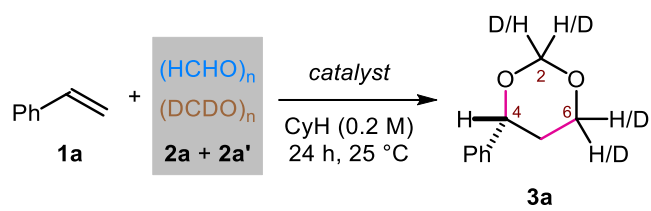

An oven-dried glass vial was charged with a mixture of paraformaldehyde $2 \mathbf{a}$ and paraformaldehyde$d_{2} \mathbf{2} \mathbf{a}$ ' (3 equiv. in total, see below), catalyst (either $2.5 \mathrm{~mol} \%$ of $(S, S)$-ilDP catalyst $\mathbf{4 b}$, or $15 \mathrm{~mol} \% p$ $\left.\mathrm{TsOH} \cdot \mathrm{H}_{2} \mathrm{O}\right)$, dry cyclohexane $(0.125 \mathrm{~mL}, 0.2 \mathrm{M})$, and styrene $1 \mathrm{a}(3 \mu \mathrm{L}, 0.025 \mathrm{mmol}, 1$ equiv.), under Ar. Then, the mixture was vigorously stirred at $\mathrm{rt}$ for $24 \mathrm{~h}$. The reaction was stopped by adding one drop of $\mathrm{Et}_{3} \mathrm{~N}$, and the mixture was diluted with $\mathrm{CDCl}_{3}$ and analyzed by ${ }^{1} \mathrm{H} \mathrm{NMR}$.

The following experiments were performed:

- Only $(\mathrm{HCHO})$ n: 3 equiv. $2 a(2.25 \mathrm{mg}, 75 \mu \mathrm{mol})$

- $\quad(\mathrm{HCHO}) \mathrm{n} /(\mathrm{DCDO}) \mathrm{n}: 2: 1: 2$ equiv. $2 \mathrm{a}(1.50 \mathrm{mg}, 50 \mu \mathrm{mol})+1$ equiv. 2a' $(0.80 \mathrm{mg}, 25 \mu \mathrm{mol})$

- $\quad(\mathrm{HCHO})_{\mathrm{n}} /(\mathrm{DCDO})_{\mathrm{n}}: 1: 1: 1.5$ equiv. $2 \mathrm{2a}(1.50 \mathrm{mg}, 37.5 \mu \mathrm{mol})+1.5$ equiv. 2a' (0.80 mg, $\left.37.5 \mu \mathrm{mol}\right)$

- $\quad(\mathrm{HCHO})_{\mathrm{n}} /(\mathrm{DCDO})_{\mathrm{n}}: 1: 2 \mathrm{:} 2$ equiv. $\mathbf{2 a}(1.50 \mathrm{mg}, 25 \mu \mathrm{mol})+1$ equiv. 2a' $(0.80 \mathrm{mg}, 50 \mu \mathrm{mol})$

- Only (DCDO)n: 3 equiv. 2a' $(2.40 \mathrm{mg}, 75 \mu \mathrm{mol})$

For the ${ }^{1} \mathrm{H}$ NMR analysis, the signal corresponding to the hydrogen atom of the product on C-4 (4.65 $\mathrm{ppm}$ ) is used in all cases as integration reference (integral value of 1.00), since that atom is not expected to be replaced by deuterium during the reaction.

Table S-3. ${ }^{1} \mathrm{H}$ NMR analysis of the Prins reaction of styrene 1a with mixtures of paraformaldehyde (2a) and paraformaldehyde$d_{2}\left(2 a^{3}\right)$.

\begin{tabular}{|c|c|c|c|c|c|c|c|c|c|}
\hline \multirow[b]{2}{*}{ Catalyst } & \multirow[b]{2}{*}{$2 a / 2 a^{\prime}$} & \multicolumn{7}{|c|}{${ }^{1} \mathrm{H}$ NMR Integrals } & \multirow[b]{2}{*}{$\begin{array}{l}\text { Ratio } \\
\text { H-2/H-6 }\end{array}$} \\
\hline & & $\mathrm{H}-4$ & $\mathrm{H}-2 \mathrm{a}$ & $\mathrm{H}-2 \mathrm{~b}$ & $\mathrm{H}-6 \mathrm{a}$ & $H-6 b$ & $\begin{array}{l}\mathrm{H}-2: \\
\mathrm{H}-2 \mathrm{a}+ \\
\mathrm{H}-2 \mathrm{~b}\end{array}$ & $\begin{array}{l}\mathrm{H}-6: \\
\mathrm{H}-6 \mathrm{a}+ \\
\mathrm{H}-6 \mathrm{~b}\end{array}$ & \\
\hline \multirow{5}{*}{ iDP 4b } & Only 2a & 1.00 & 0.96 & 1.11 & 1.02 & 1.03 & 2.07 & 2.05 & 1.01 \\
\hline & $2: 1$ & 1.00 & 0.80 & 0.90 & 0.84 & 0.83 & 1.70 & 1.67 & 1.02 \\
\hline & $1: 1$ & 1.00 & 0.70 & 0.80 & 0.73 & 0.73 & 1.49 & 1.47 & 1.02 \\
\hline & $1: 2$ & 1.00 & 0.44 & 0.54 & 0.54 & 0.54 & 0.97 & 1.08 & 0.90 \\
\hline & Only 2a' & 1.00 & 0.00 & 0.00 & 0.00 & 0.00 & 0.00 & 0.00 & $n / a$ \\
\hline & & & & & & & & & \\
\hline \multirow{5}{*}{$p$-TsOH } & Only 2a & 1.00 & 0.98 & 1.08 & 0.98 & 0.99 & 2.06 & 1.97 & 1.05 \\
\hline & $2: 1$ & 1.00 & 0.61 & 0.65 & 0.98 & 1.00 & 1.26 & 1.98 & 0.64 \\
\hline & $1: 1$ & 1.00 & 0.44 & 0.47 & 0.96 & 0.99 & 0.91 & 1.95 & 0.47 \\
\hline & $1: 2$ & 1.00 & 0.31 & 0.34 & 0.70 & 0.71 & 0.65 & 1.41 & 0.46 \\
\hline & Only 2a' & 1.00 & 0.00 & 0.00 & 0.00 & 0.00 & 0.00 & 0.00 & $n / a$ \\
\hline
\end{tabular}

As can be seen in Table S-3, the experiments with ilDP $\mathbf{4 b}$ as catalyst led to a 1,3-dioxane product with very similar content of ${ }^{1} \mathrm{H}$ on positions $\mathrm{C}-2$ and $\mathrm{C}-\mathbf{6}$, regardless of the $\mathbf{2} \mathbf{a} / \mathbf{2} \mathbf{a}$ ' ratio. This points towards a (pseudo)-concerted reaction pathway, where both formaldehyde units come from the same paraformaldehyde chain.

Conversely, the use of $p-\mathrm{TsOH}$ as catalyst resulted in the formation of a product with different ${ }^{1} \mathrm{H}$ contents on positions $\mathrm{C}-2$ and $\mathrm{C}-6$, which could be understood as the reaction proceeding rather via a stepwise pathway. Also, when $\mathbf{2} \mathbf{a} / \mathbf{2} \mathbf{a}^{\prime}$ mixtures were used, the lower ${ }^{1} \mathrm{H}$ incorporation on position $\mathrm{C}-2$ 
in comparison to C-6 might indicate a stronger kinetic isotope effect for the acetalization step than for the olefin-aldehyde $\mathrm{C}-\mathrm{C}$ bond-forming step.

\subsection{Stereospecificity: experiments with $\beta$-deutero-styrenes}

Two extreme scenarios can be considered for the mechanism of the ilDP-catalyzed Prins reaction: either a stepwise reaction pathway (involving the intermediacy of a benzyl cation), or a (pseudo)concerted pathway. The previously shown experiments with $(\mathrm{HCHO})_{n} /(\mathrm{DCDO})_{n}$ mixtures (see Section 6.1 of this Supporting Information file) point towards the latter case being the operative pathway. We considered that this could be further confirmed by studying the reaction with $\beta$-deuterostyrenes (both trans- and cis- isomers: $\mathbf{1 q}$ and $\mathbf{1 r}$, respectively) as substrates. Similarly to the $(\mathrm{HCHO})_{n} /(\mathrm{DCDO})_{n}$ experiment, either ilDP $\mathbf{4 b}$ or $p-\mathrm{TsOH}$ were used as catalysts.

For the iDP-catalyzed reactions, the stereochemistry of the starting olefin was translated to the 1,3dioxane product, as could be seen on the ${ }^{1} \mathrm{H}$ NMR spectra $(\mathrm{H}-4$ and $\mathrm{H}-5$, see Scheme S-2): the cisolefin was transformed solely to a cis-product; the trans-olefin resulted in the exclusive formation of a trans-product.

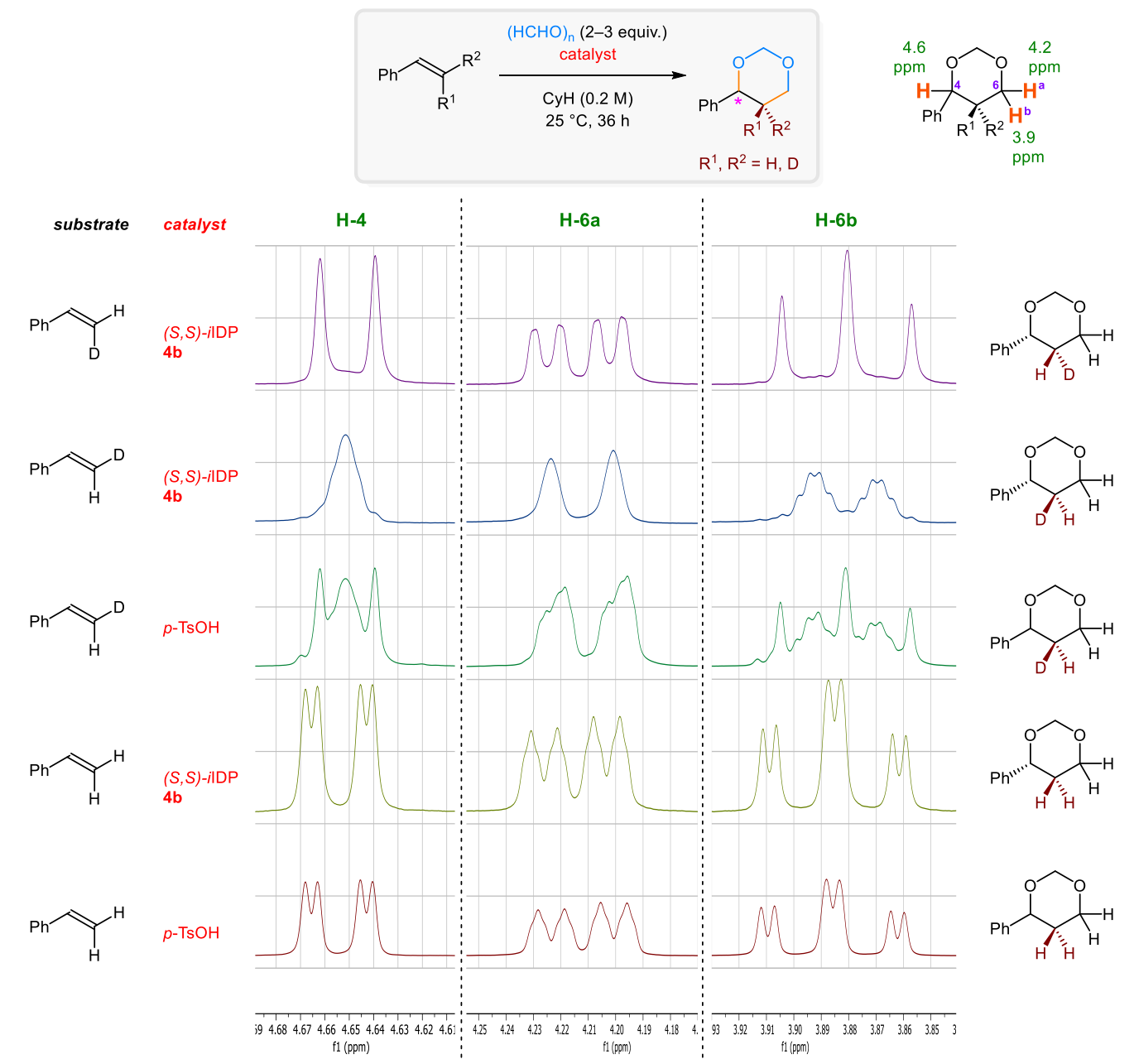

Scheme S-2. ${ }^{1} \mathrm{H}$ NMR analysis of the Prins reaction of $\beta$-deutero-styrenes (1 w, 1x) with paraformaldehyde (2a).

However, when the reaction was catalyzed with $p-\mathrm{TsOH}$, the obtained product corresponded rather to an equimolar mixture of cis- and trans-1,3-dioxanes. These differences in the outcome of the reaction are in concordance with the previous mechanistic studies, underlining the different operating pathways depending on the type of catalyst used.

The results from the reaction with $\beta$-deutero-styrenes can be understood like this: 
- When $p-\mathrm{TsOH}$ is used as catalyst, the reaction proceeds via a benzyl cation intermediate, which can rotate around the $\mathrm{C}-\mathrm{C}$ bond, giving rise to the mixture of cis- and trans-products, regardless of the stereochemistry from the starting olefin.

- The iDP-catalyzed reaction is probably proceeding rather via a concerted pathway, which makes the stereoinformation of the starting olefin to be translated into the formed 1,3-dioxane.

\subsection{EWG on the catalyst structure: higher reactivity}

To better understand the effect of the electron-withdrawing groups introduced in the catalyst structure (from $\mathbf{4 a}$ to $\mathbf{4 b}$, and then to $\mathbf{4} \mathbf{b}^{\text {') }}$, we conducted a ${ }^{1} \mathrm{H}$ NMR monitoring of the Prins reaction of styrene (1a) with paraformaldehyde (2a) toward 4-phenyl-1,3-dioxane (3a), using each of the mentioned iDP catalysts $(2.5 \mathrm{~mol} \%)$, in $\mathrm{CDCl}_{3}$ at $\mathrm{rt}$, in the presence of $\mathrm{Ph}_{3} \mathrm{CH}$ as internal standard for quantification.

As can be seen in Figure S-2, exchanging the 3,3'-substituent of the catalyst ( $\left.R^{\mathrm{W}}\right)$ from an alkylsubstituted to an EWG-substituted phenyl ring translates into a significant increase in reactivity. Further introduction of EWGs on the structure, now on the 6,6'-positions of the BINOL backbone $\left(R^{B}\right)$, results in higher reactivity.
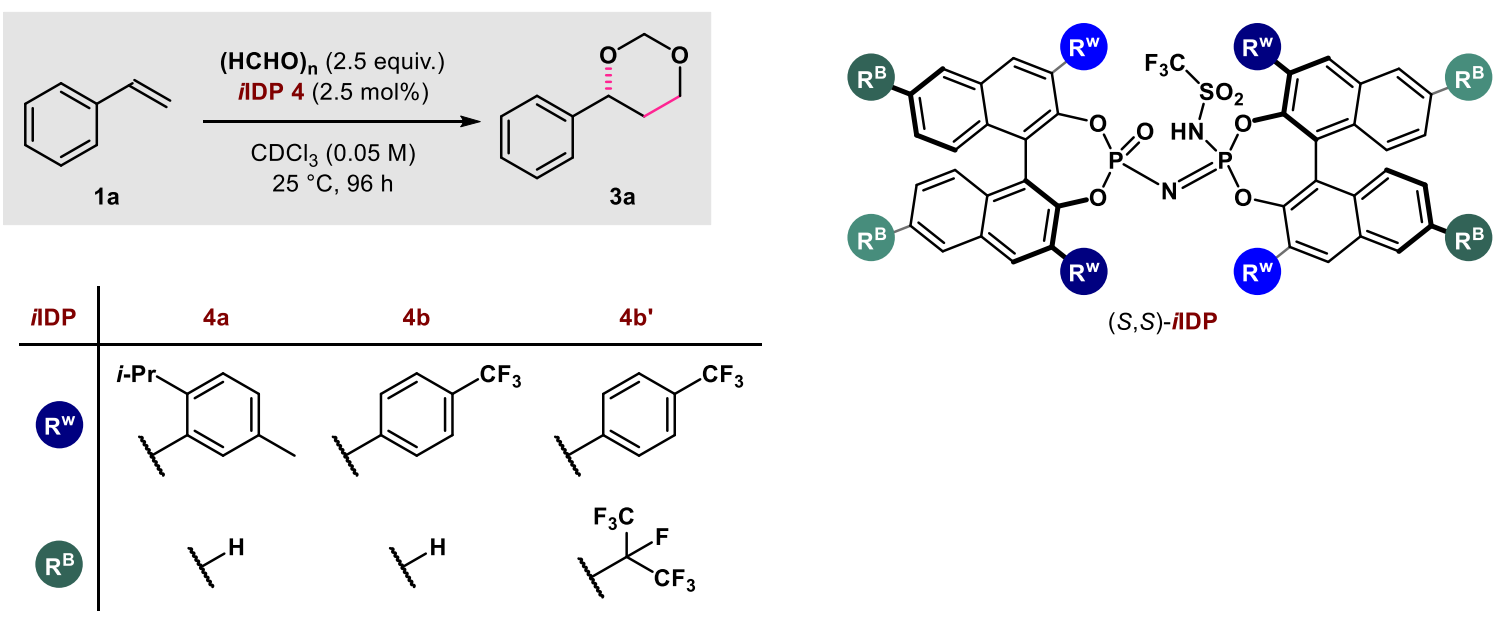

$(S, S)$-ilDP

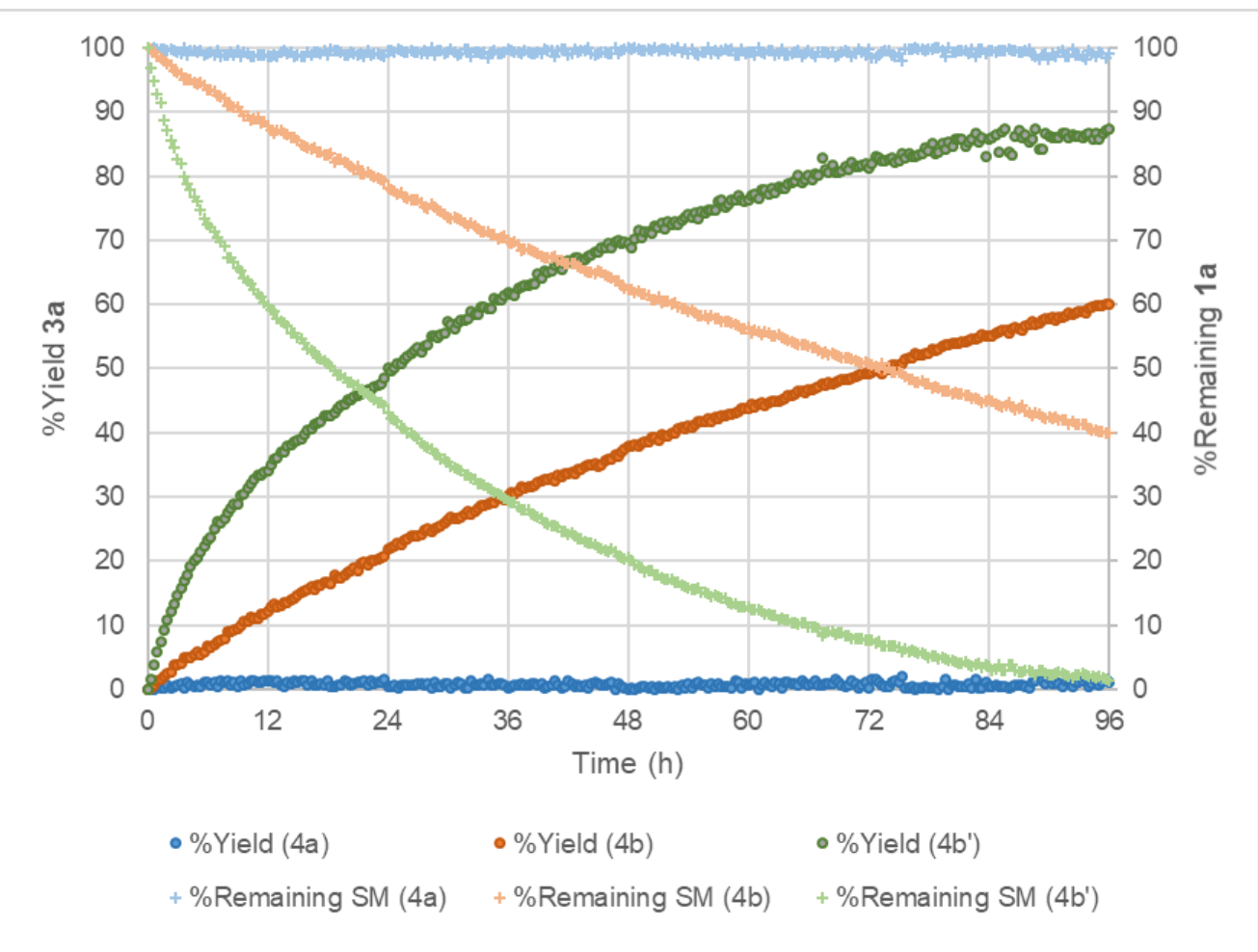

Figure S-2. ${ }^{1} \mathrm{H}$ NMR monitoring for the model Prins reaction using catalysts $4 \mathbf{a}, \mathbf{4 b}$ and $\mathbf{4} \boldsymbol{b}^{\prime}$ (in $\mathrm{CDCl}_{3}$ at $25^{\circ} \mathrm{C}$ ). 


\section{Synthesis of BINOL-derived imino-imidodiphosphates (IDPs)}

The synthesis of BINOL-derived imino-imidodiphosphates requires 3,3'-disubstituted BINOLs, which are prepared by means of a palladium-catalyzed Suzuki cross coupling reactions: the boronate resides on the BINOL moiety and the 3,3' substituents are inserted as (pseudo)halides, whose preparation is described below:

\section{2-bromo-4-iodo-1-(trifluoromethyl)benzene (S5)}

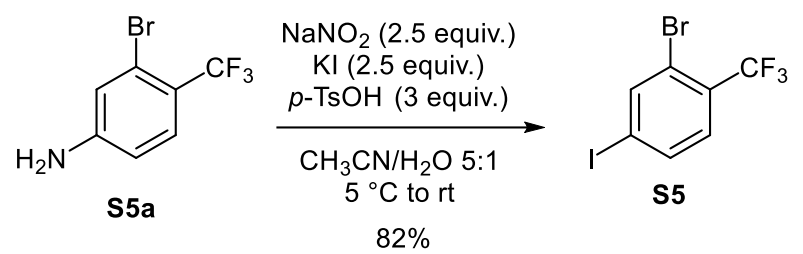

(Adapted from a reported procedure ${ }^{20}$ ) A RB flask under air was charged with 3-bromo-4(trifluoromethyl)aniline S5a (3.0 g, $15 \mathrm{mmol}, 1$ equiv.), acetonitrile $(65 \mathrm{~mL})$ and $p$-toluenesulfonic acid monohydrate ( $7.13 \mathrm{~g}, 45 \mathrm{mmol}, 3$ equiv.), and the mixture was cooled to $0-5{ }^{\circ} \mathrm{C}$ (ice-water bath). A solution of sodium nitrite $(1.72 \mathrm{~g}, 30 \mathrm{mmol}, 2$ equiv.) and potassium iodide $(5.19 \mathrm{~g}, 37.5 \mathrm{mmol}, 2.5$ equiv.) in distilled water $(12 \mathrm{~mL})$ is added dropwise over $5 \mathrm{~min}$. The mixture was allowed to reach $\mathrm{rt}$ and further stirred for $30 \mathrm{~min}$, then poured onto distilled water $(30 \mathrm{~mL})$, neutralized with satd. aq. $\mathrm{NaHCO}_{3}(20 \mathrm{~mL})$ and extracted with MTBE $(3 \times 30 \mathrm{~mL})$. The combined organic phases were washed with satd. aq. $\mathrm{NaHCO}_{3}(1 \times 20 \mathrm{~mL})$, aq. $\mathrm{Na}_{2} \mathrm{~S}_{2} \mathrm{O}_{3} 10 \%(1 \times 20 \mathrm{~mL})$, water $(1 \times 20 \mathrm{~mL})$ and brine $(1 \times 20$ $\mathrm{mL}$ ), successively; then dried over anhydrous $\mathrm{Na}_{2} \mathrm{SO}_{4}$, filtered and concentrated under reduced pressure. Purification by flash column chromatography on silica gel ( $n$-pentane) afforded the corresponding aryl iodide $\mathbf{S} 5$ as a colorless liquid $(4.32 \mathrm{~g}, 82 \%)$.

${ }^{1} \mathrm{H}$ NMR $\left(501 \mathrm{MHz}, \mathrm{CDCl}_{3}\right) \delta 8.09$ (d, $\left.J=0.9 \mathrm{~Hz}, 1 \mathrm{H}\right), 7.76$ (dd, $\left.J=8.3,0.9 \mathrm{~Hz}, 1 \mathrm{H}\right), 7.38(\mathrm{~d}, J=8.3$ $\mathrm{Hz}, 2 \mathrm{H})$.

${ }^{19} \mathrm{~F} \mathrm{NMR}\left(471 \mathrm{MHz}, \mathrm{CDCl}_{3}\right) \delta-62.90$ (s, 3F).

${ }^{13} \mathrm{C} \mathrm{NMR}\left(126 \mathrm{MHz}, \mathrm{CDCl}_{3}\right) \delta 143.2(\mathrm{CH}), 136.7(\mathrm{CH}), 130.0(\mathrm{~d}, J=31.9 \mathrm{~Hz}, \mathrm{C}), 129.0(\mathrm{q}, J=5.2 \mathrm{~Hz}$, $\mathrm{CH}$ ), 123.0 (q, $J=273.4 \mathrm{~Hz}, \mathrm{C}$ ), 121.0 (C), 98.9 (C). Spectroscopic data was consistent with the values reported in the literature. ${ }^{21}$

El-HRMS: calculated for $\mathrm{C}_{7} \mathrm{H}_{3} \mathrm{BrF}_{3} l^{+}\left([\mathrm{M}]^{+}\right): 349.8410$, found: 349.8409 .

\section{6-(perfluoropropan-2-yl)naphthalen-2-yl trifluoromethanesulfonate (S6)}

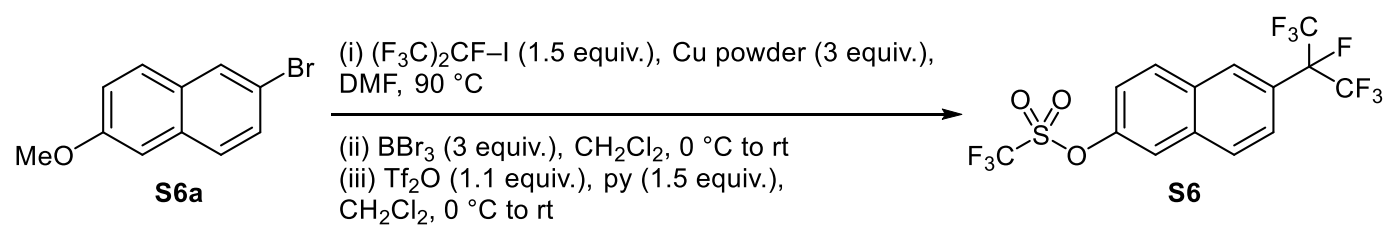

$72 \%$ over three steps

2-methoxy-6-(perfluoropropan-2-yl)naphthalene:

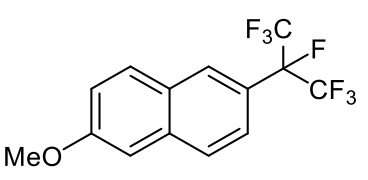

An oven-dried Schlenk flask under $\mathrm{Ar}$ atmosphere was charged with $\mathrm{Cu}$ powder $(2.86 \mathrm{~g}, 45 \mathrm{mmol}, 3$ equiv.; freshly activated with aq. $\mathrm{HCl} 2 \mathrm{M}$, washed with ethanol and acetone, and dried under vacuum overnight), 6bromo-2-methoxynaphthalene (S6a, $3.56 \mathrm{~g}, 15 \mathrm{mmol}, 1$ equiv.), and dry DMF (25 mL). After degassing the mixture by bubbling Ar for $5 \mathrm{~min}$, perfluoroisopropyl iodide $(3.2 \mathrm{~mL}$, $22.5 \mathrm{mmol}, 1.5$ equiv.) was added in one portion and the flask was closed under Ar. The mixture was stirred vigorously at $90{ }^{\circ} \mathrm{C}$ (oil bath) for $24 \mathrm{~h}$. After cooling to rt, the mixture was diluted with MTBE $(100 \mathrm{~mL})$, treated with water $(1 \mathrm{~mL}$ dropwise, then $20 \mathrm{~mL}$ in one portion) and vigorously stirred for 10 
min at rt. Afterwards, the mixture was passed through a Celite ${ }^{\circledR}$ pad, washing with MTBE. The aqueous layer was further extracted with MTBE $(3 \times 50 \mathrm{~mL})$ and the combined organic layers were washed with aq. $\mathrm{HCl} 2 \mathrm{M}(1 \times 30 \mathrm{~mL})$, water $(1 \times 100 \mathrm{~mL})$ and brine $(1 \times 50 \mathrm{~mL})$. After drying over anhydrous $\mathrm{Na}_{2} \mathrm{SO}_{4}$, the organic phase was filtered and concentrated under reduced pressure, affording a yellow liquid, which was used directly for the next reaction.

${ }^{1} \mathrm{H}$ NMR (501 MHz, CDCl $)$ ): $\delta 8.04(\mathrm{~s}, 1 \mathrm{H}), 7.83(\mathrm{t}, J=9.4 \mathrm{~Hz}, 2 \mathrm{H}), 7.59(\mathrm{~d}, J=8.1 \mathrm{~Hz}, 1 \mathrm{H}), 7.24$ (dd, $J$ $=8.9,2.6 \mathrm{~Hz}, 1 \mathrm{H}), 7.17(\mathrm{~d}, J=2.5 \mathrm{~Hz}, 1 \mathrm{H}), 3.95(\mathrm{~s}, 3 \mathrm{H})$.

${ }^{19} \mathrm{~F} \mathrm{NMR}\left(471 \mathrm{MHz}, \mathrm{CDCl}_{3}\right) \delta-75.51$ (d, $\left.J=7.3 \mathrm{~Hz}, 6 \mathrm{~F}\right),-181.74$ (hept, $J=7.4 \mathrm{~Hz}, 1 \mathrm{~F}$ ).

\section{6-(perfluoropropan-2-yl)naphthalen-2-ol:}

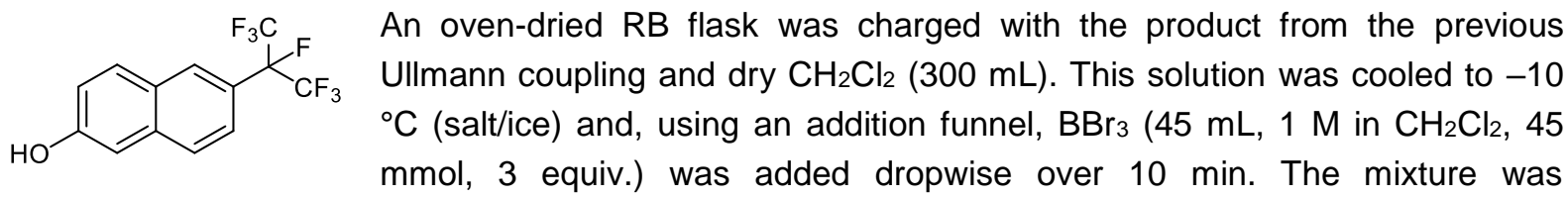
vigorously stirred at $-10^{\circ} \mathrm{C}$ for $30 \mathrm{~min}$, and then at $\mathrm{rt}$ for $6 \mathrm{~h}$. After cooling to $0{ }^{\circ} \mathrm{C}$, the reaction was stopped by dropwise addition of $\mathrm{MeOH}(50 \mathrm{~mL}$, with the addition funnel) and further diluted with distilled water $(200 \mathrm{~mL})$. The aqueous layer was extracted with $\mathrm{CH}_{2} \mathrm{Cl}_{2}(2 \times 50 \mathrm{~mL})$, and the combined organic phases were washed with brine $(1 \times 100 \mathrm{~mL})$. After drying over anhydrous $\mathrm{Na}_{2} \mathrm{SO}_{4}$, the organic phase was filtered and concentrated under reduced pressure, affording an off-white solid, which was used directly for the next reaction.

${ }^{1} \mathrm{H}$ NMR $\left(501 \mathrm{MHz}, \mathrm{CD}_{2} \mathrm{Cl}_{2}\right) \delta 8.18(\mathrm{~s}, 1 \mathrm{H}), 8.01(\mathrm{~d}, J=8.9 \mathrm{~Hz}, 1 \mathrm{H}), 7.98(\mathrm{~d}, J=8.9 \mathrm{~Hz}, 1 \mathrm{H}), 7.69(\mathrm{~d}, J$ $=8.8 \mathrm{~Hz}, 1 \mathrm{H}), 7.58(\mathrm{~d}, J=2.5 \mathrm{~Hz}, 1 \mathrm{H}), 7.34(\mathrm{dd}, J=8.9,2.4 \mathrm{~Hz}, 1 \mathrm{H})$.

${ }^{19} \mathrm{~F} \mathrm{NMR}\left(471 \mathrm{MHz}, \mathrm{CD}_{2} \mathrm{Cl}_{2}\right) \delta-75.76(\mathrm{~d}, J=6.8 \mathrm{~Hz}, 6 \mathrm{~F}),-182.06$ (hept, $J=7.1 \mathrm{~Hz}, 1 \mathrm{~F}$ ).

\section{6-(perfluoropropan-2-yl)naphthalen-2-yl trifluoromethanesulfonate (S6):}

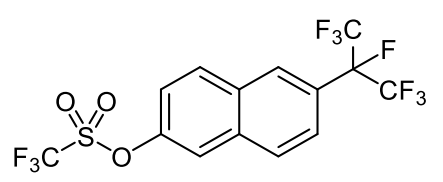

An oven-dried RB flask was charged with the product from the previous deprotection and dry $\mathrm{CH}_{2} \mathrm{Cl}_{2}(40 \mathrm{~mL})$. This solution was cooled to $0{ }^{\circ} \mathrm{C}$ (ice/water) and treated with pyridine (1.8 mL, $22.5 \mathrm{mmol}, 1.5$ equiv.). Trifluoromethanesulfonic anhydride $(2.8 \mathrm{~mL}, 16.5 \mathrm{mmol}, 1.1$ equiv.) was added dropwise at $0{ }^{\circ} \mathrm{C}$ and the mixture was stirred vigorously at $\mathrm{rt}$ overnight. The reaction was stopped by dropwise addition of aq. $\mathrm{HCl} 1 \mathrm{M}(50 \mathrm{~mL})$ and further diluted with MTBE $(100 \mathrm{~mL})$. The aqueous layer was extracted with MTBE $(2 \times 30 \mathrm{~mL})$, and the combined organic phases were washed subsequently with satd. aq. $\mathrm{NaHCO}_{3}(1 \times 50 \mathrm{~mL})$, water $(1 \times 30 \mathrm{~mL})$, and brine $(1 \times 30 \mathrm{~mL})$. After drying over anhydrous $\mathrm{Na}_{2} \mathrm{SO}_{4}$, the organic phase was filtered and concentrated under reduced pressure. Purification by flash column chromatography on silica gel ( $n$-pentane) afforded the corresponding aryl triflate $\mathbf{S 6}$ as a light yellow oil that solidified upon standing as white solid $(4.78 \mathrm{~g}$, $72 \%$ over three steps).

${ }^{1} \mathrm{H} \mathrm{NMR}\left(501 \mathrm{MHz}, \mathrm{CD}_{2} \mathrm{Cl}_{2}\right) \delta 8.25(\mathrm{~s}, 1 \mathrm{H}), 8.09(\mathrm{~d}, J=9.1 \mathrm{~Hz}, 1 \mathrm{H}), 8.07(\mathrm{~d}, J=8.9 \mathrm{~Hz}, 1 \mathrm{H}), 7.88(\mathrm{~d}, J$ $=2.5 \mathrm{~Hz}, 1 \mathrm{H}), 7.77(\mathrm{~d}, J=8.8 \mathrm{~Hz}, 1 \mathrm{H}), 7.53(\mathrm{dd}, J=9.0,2.5 \mathrm{~Hz}, 1 \mathrm{H})$.

${ }^{19} \mathrm{~F} \mathrm{NMR}\left(471 \mathrm{MHz}, \mathrm{CD}_{2} \mathrm{Cl}_{2}\right.$ ) $\delta-73.10$ (s, 3F), -75.73 (d, $J=7.4 \mathrm{~Hz}, 6 \mathrm{~F}$ ), -182.12 (hept, $J=7.2 \mathrm{~Hz}$, $1 F)$.

${ }^{13} \mathrm{C}$ NMR (126 MHz, $\left.\mathrm{CD}_{2} \mathrm{Cl}_{2}\right) \delta$ 149.0, 134.7, 132.2, 132.0 (d, $\left.J=2.3 \mathrm{~Hz}\right), 130.0$ (d, $\left.J=2.4 \mathrm{~Hz}\right), 127.0$ (d, $J=12.2 \mathrm{~Hz}$ ), 125.8 (d, $J=20.5 \mathrm{~Hz}), 123.7(\mathrm{~d}, J=9.7 \mathrm{~Hz}), 122.2(\mathrm{~d}, J=28.1 \mathrm{~Hz}), 121.6,119.92(\mathrm{~d}$, $J=27.5 \mathrm{~Hz}), 119.63,119.2(\mathrm{~d}, J=320.9 \mathrm{~Hz})$.

El-HRMS: calculated for $\mathrm{C}_{14} \mathrm{H}_{6} \mathrm{~F}_{10} \mathrm{O}_{3} \mathrm{~S}^{+}\left([\mathrm{M}]^{+}\right): 443.9873$, found: 443.9880 . 


\section{4-(perfluoropropyl)phenyl trifluoromethanesulfonate (S7)}

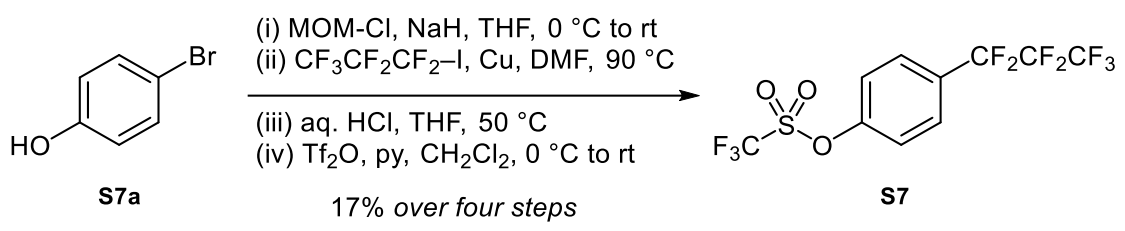

1-bromo-4-(methoxymethoxy)benzene:

4-bromophenol (S7a, $3.46 \mathrm{~g}, 20 \mathrm{mmol}, 1$ equiv.) and dry THF (65 mL). To the
cooled mixture at $0{ }^{\circ} \mathrm{C}, \mathrm{NaH}(960 \mathrm{mg}$ of $60 \%$ suspension in oil, $24 \mathrm{mmol}, 1.2$ equiv.) was added portionwise and, after stirring $15 \mathrm{~min}, \mathrm{MOM}-\mathrm{Cl}(1.8 \mathrm{~mL}, 22 \mathrm{mmol}, 1.1$ equiv.) was added in one portion. The mixture was slowly warmed up to $\mathrm{rt}$ and further stirred for $24 \mathrm{~h}$. The reaction was quenched by carefully adding satd. aq. $\mathrm{NH}_{4} \mathrm{Cl}$, diluted with $50 \mathrm{~mL}$ distilled water and extracted with MTBE $(3 \times 25 \mathrm{~mL})$. Then, the combined organic phase was washed with brine $(1 \times 30 \mathrm{~mL})$, dried over anhydrous $\mathrm{Na}_{2} \mathrm{SO}_{4}$, filtered and concentrated under reduced pressure. The obtained crude was directly used for the next step, without further purification.

${ }^{1} \mathrm{H}$ NMR $\left(501 \mathrm{MHz}, \mathrm{CD}_{2} \mathrm{Cl}_{2}\right) \delta 7.39(\mathrm{~d}, J=9.0 \mathrm{~Hz}, 2 \mathrm{H}), 6.93(\mathrm{~d}, J=9.0 \mathrm{~Hz}, 2 \mathrm{H}), 5.14(\mathrm{~s}, 2 \mathrm{H}), 3.44$ (s, $3 \mathrm{H})$.

${ }^{13} \mathrm{C}$ NMR $\left(126 \mathrm{MHz}, \mathrm{CD}_{2} \mathrm{Cl}_{2}\right) \delta 156.9,132.6,118.6,114.3,95.0,56.3$.

Spectroscopic data was consistent with the values reported in the literature. ${ }^{22}$

1-(methoxymethoxy)-4-(perfluoropropyl)benzene:<smiles>COCOc1ccc(C(F)(F)C(F)(F)F)cc1</smiles>

An oven-dried Schlenk flask under Ar atmosphere was charged with $\mathrm{Cu}$ powder $(1.90 \mathrm{~g}, 30 \mathrm{mmol}, 6$ equiv.; freshly activated with aq. $\mathrm{HCl} 2 \mathrm{M}$, washed with ethanol and acetone, and dried under vacuum overnight), crude 1-bromo-4-(methoxymethoxy)benzene (1.08 g, $5 \mathrm{mmol}, 1$ equiv.), and dry DMF (10 mL). After degassing the mixture by bubbling Ar for $5 \mathrm{~min}$, perfluoropropyl iodide (2.2 $\mathrm{mL}, 15 \mathrm{mmol}, 3$ equiv.) was added in one portion and the flask was closed under Ar. The mixture was stirred vigorously at $90^{\circ} \mathrm{C}$ (oil bath) for 2.5 days. After cooling to rt, the mixture was diluted with MTBE $(30 \mathrm{~mL})$, treated with water ( $1 \mathrm{~mL}$ dropwise, then $5 \mathrm{~mL}$ in one portion) and vigorously stirred for $10 \mathrm{~min}$ at rt. Afterwards, the mixture was passed through a Celite $\AA$ pad, washing with MTBE. The aqueous layer was further extracted with MTBE $(3 \times 30 \mathrm{~mL})$ and the combined organic layers were washed with water $(1 \times 100 \mathrm{~mL})$ and brine $(1 \times 50 \mathrm{~mL})$. After drying over anhydrous $\mathrm{Na}_{2} \mathrm{SO}_{4}$, the organic phase was filtered and concentrated under reduced pressure, affording a light yellow liquid, which was used directly for the next reaction.

${ }^{1} \mathrm{H}$ NMR (501 MHz, $\mathrm{CD}_{2} \mathrm{Cl}_{2}$ ) $\delta 7.51$ (d, $J=8.8 \mathrm{~Hz}, 2 \mathrm{H}$ ), 7.15 (d, $J=8.9 \mathrm{~Hz}, 2 \mathrm{H}$ ), $5.23(\mathrm{~s}, 2 \mathrm{H}$ ), 3.47 (s, $3 \mathrm{H})$.

${ }^{19} \mathrm{~F}$ NMR $\left(471 \mathrm{MHz}, \mathrm{CD}_{2} \mathrm{Cl}_{2}\right) \delta-80.42(\mathrm{t}, J=10.0 \mathrm{~Hz}, 3 \mathrm{~F}),-110.96(\mathrm{q}, J=10.2 \mathrm{~Hz}, 2 \mathrm{~F}),-126.75(\mathrm{~s}$, $2 \mathrm{~F})$.

${ }^{13} \mathrm{C}$ NMR (126 MHz, $\left.\mathrm{CD}_{2} \mathrm{Cl}_{2}\right) \delta 160.6(\mathrm{C}), 128.7(\mathrm{t}, J=6.6 \mathrm{~Hz}, \mathrm{CH}), 116.6(\mathrm{CH}), 94.7\left(\mathrm{CH}_{2}\right), 56.5$ $\left(\mathrm{CH}_{3}\right)$.

4-(perfluoropropyl)phenol:<smiles>Oc1ccc(C(F)(F)C(F)(F)F)cc1</smiles>

A RB flask was charged with the crude product from the previous Ullmann coupling (1.18 g, 3.8 mmol), 1,4-dioxane (8 mL), $\mathrm{MeOH}(1 \mathrm{~mL})$, and aq. $\mathrm{HCl} 6$ $\mathrm{M}\left(3 \mathrm{~mL}, 18 \mathrm{mmol}, 5\right.$ equiv.). The mixture was heated to $90^{\circ} \mathrm{C}$ overnight and, after cooling to rt, it was diluted with $\mathrm{CH}_{2} \mathrm{Cl}_{2}(50 \mathrm{~mL})$ and distilled water $(50 \mathrm{~mL})$.

The aqueous layer was further extracted with $\mathrm{CH}_{2} \mathrm{Cl}_{2}(3 \times 20 \mathrm{~mL})$ and the combined organic layers were washed with brine $(1 \times 30 \mathrm{~mL})$. After drying over anhydrous $\mathrm{Na}_{2} \mathrm{SO}_{4}$, the organic phase was 
filtered and concentrated under reduced pressure, affording an orange oil, which was used directly for the next reaction.

${ }^{1} \mathrm{H} \mathrm{NMR}\left(501 \mathrm{MHz}, \mathrm{CD}_{2} \mathrm{Cl}_{2}\right) \delta 7.47(\mathrm{~d}, J=8.6 \mathrm{~Hz}, 2 \mathrm{H}), 6.96(\mathrm{~d}, J=8.8 \mathrm{~Hz}, 2 \mathrm{H}), 5.57(\mathrm{~s}, 1 \mathrm{H})$. ${ }^{19} \mathrm{~F} \mathrm{NMR}\left(471 \mathrm{MHz}, \mathrm{CD}_{2} \mathrm{Cl}_{2}\right) \delta-80.44$ (t, $\left.J=9.9 \mathrm{~Hz}, 3 \mathrm{~F}\right),-110.85$ (q, $\left.J=9.9 \mathrm{~Hz}, 2 \mathrm{~F}\right),-126.83(\mathrm{~s}, 2 \mathrm{~F})$. ${ }^{13} \mathrm{C}$ NMR $\left(126 \mathrm{MHz}, \mathrm{CD}_{2} \mathrm{Cl}_{2}\right) \delta 159.3(\mathrm{C}), 129.0(\mathrm{t}, J=6.5 \mathrm{~Hz}, \mathrm{CH}), 116.0(\mathrm{CH})$.

4-(perfluoropropyl)phenyl trifluoromethanesulfonate (S7):<smiles>O=S(=O)(Oc1ccc(F)cc1)Oc1ccc(C(F)(F)F)cc1</smiles>

An oven-dried RB flask was charged with crude 4-(perfluoropropyl)phenol (856 mg, $\sim 3.25 \mathrm{mmol}, 1$ equiv.) and dry $\mathrm{CH}_{2} \mathrm{Cl}_{2}(7 \mathrm{~mL})$. This solution was cooled to $0{ }^{\circ} \mathrm{C}$ (ice/water) and treated with pyridine $(0.36 \mathrm{~mL}, 4.25 \mathrm{mmol}$, 1.3 equiv.). Trifluoromethanesulfonic anhydride $(0.60 \mathrm{~mL}, 3.6 \mathrm{mmol}, 1.1$ equiv.) was added dropwise at $0{ }^{\circ} \mathrm{C}$ and the mixture was stirred vigorously at rt overnight. The reaction was stopped by dropwise addition of aq. $\mathrm{HCl} 1 \mathrm{M}(10 \mathrm{~mL})$ and further diluted with MTBE $(25 \mathrm{~mL})$. The aqueous layer was extracted with MTBE $(2 \times 20 \mathrm{~mL})$, and the combined organic phases were washed subsequently with satd. aq. $\mathrm{NaHCO}_{3}(1 \times 30 \mathrm{~mL})$, water $(1 \times 30 \mathrm{~mL})$, and brine $(1 \times 30 \mathrm{~mL})$. After drying over anhydrous $\mathrm{Na}_{2} \mathrm{SO}_{4}$, the organic phase was filtered and concentrated under reduced pressure. Purification by flash column chromatography on silica gel ( $n$-pentane) afforded the corresponding aryl triflate $\mathbf{S 7}$ as a colorless liquid (442 $\mathrm{mg}, 72 \%$ over three steps).

${ }^{1} \mathrm{H}$ NMR $\left(501 \mathrm{MHz}, \mathrm{CD}_{2} \mathrm{Cl}_{2}\right) \delta 7.74(\mathrm{~d}, J=8.8 \mathrm{~Hz}, 2 \mathrm{H}), 7.48(\mathrm{~d}, J=8.8 \mathrm{~Hz}, 2 \mathrm{H})$.

${ }^{19} \mathrm{~F} \mathrm{NMR}\left(471 \mathrm{MHz}, \mathrm{CD}_{2} \mathrm{Cl}_{2}\right) \delta-73.06(\mathrm{~s}, 3 \mathrm{~F}),-80.35$ (t, $\left.J=10.0 \mathrm{~Hz}, 3 \mathrm{~F}\right),-111.86(\mathrm{q}, J=10.0 \mathrm{~Hz}, 2 \mathrm{~F})$, $-126.53(\mathrm{~s}, 2 \mathrm{~F})$.

${ }^{13} \mathrm{C}$ NMR (126 MHz, $\left.\mathrm{CD}_{2} \mathrm{Cl}_{2}\right) \delta 152.4(\mathrm{C}), 129.7$ (t, $\left.J=6.5 \mathrm{~Hz}, \mathrm{CH}\right), 122.5(\mathrm{CH}), 119.1$ (q, $J=320.7$ $\mathrm{Hz}, \mathrm{C})$.

APCl-HRMS: calculated for $\mathrm{C}_{10} \mathrm{H}_{4} \mathrm{~F}_{10} \mathrm{O}_{3} \mathrm{~S}^{+}\left([\mathrm{M}]^{+}\right)$: 393.9716, found: 393.9716 .

\subsection{Synthesis of 3,3'-disubstituted BINOLs}

\section{General Procedure E: Suzuki Coupling and MOM Deprotection}

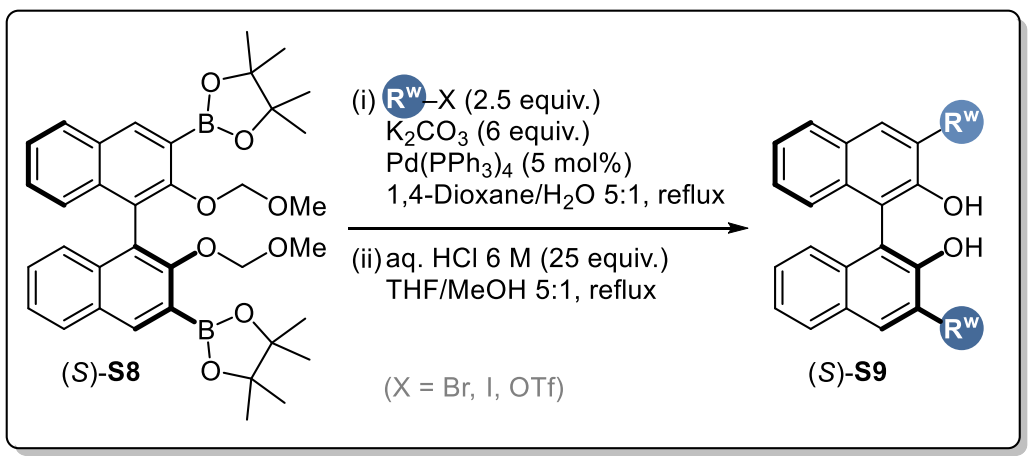

In a flask under $\operatorname{Ar}$, (S)-2,2'-(2,2'-bis(methoxymethoxy)-[1,1'-binaphthalene]-3,3'-diyl)bis(4,4,5,5tetramethyl-1,3,2-dioxaborolane) $\mathbf{S 8}^{23}$ (313 $\mathrm{mg}, 0.50 \mathrm{mmol}, 1$ equiv.), the corresponding aryl (pseudo)halide (2-3 equiv.), and solid $\mathrm{K}_{2} \mathrm{CO}_{3}(415 \mathrm{mg}, 3 \mathrm{mmol}, 6$ equiv.) were dissolved in a 4:1 $\mathrm{v} / \mathrm{v}$ mixture of 1,4-dioxane/water $(5 \mathrm{~mL})$. After degassing the mixture by bubbling Ar for $5 \mathrm{~min}, \mathrm{Pd}\left(\mathrm{PPh}_{3}\right)_{4}$ (29 mg, $0.025 \mathrm{mmol}, 5 \mathrm{~mol} \%$ ) was added and the resulting mixture was heated to reflux for $18-24 \mathrm{~h}$. The reaction mixture was cooled to rt and filtered through a short pad of Celite®, washing with $\mathrm{CH}_{2} \mathrm{Cl}_{2}$ $(30 \mathrm{~mL})$. The filtrate was washed with water $(1 \times 20 \mathrm{~mL})$ and brine $(1 \times 20 \mathrm{~mL})$, then dried over anhydrous $\mathrm{Na}_{2} \mathrm{SO}_{4}$, filtered and concentrated under reduced pressure.

The obtained crude was redissolved in a mixture of THF/MeOH 3:1 v/v $(10 \mathrm{~mL})$, treated with aq. $\mathrm{HCl} 6$ $\mathrm{M}\left(2 \mathrm{~mL}, 12 \mathrm{mmol}, 24\right.$ equiv.) and heated to $50{ }^{\circ} \mathrm{C}$ overnight. After cooling to rt, the mixture was 
diluted with $\mathrm{CH}_{2} \mathrm{Cl}_{2}(20 \mathrm{~mL})$ and water $(20 \mathrm{~mL})$, and the aqueous phase was further extracted with $\mathrm{CH}_{2} \mathrm{Cl}_{2}(3 \times 20 \mathrm{~mL})$. The combined organic phases were washed with brine $(1 \times 30 \mathrm{~mL})$ and, after drying over anhydrous $\mathrm{Na}_{2} \mathrm{SO}_{4}$, the extract was filtered and concentrated under reduced pressure. Purification by flash column chromatography on silica gel (n-pentane $/ \mathrm{CH}_{2} \mathrm{Cl}_{2}$ or $n$-pentane/MTBE mixtures) afforded the corresponding 3,3'-disubstituted BINOL (S)-S9.

\section{(S)-3,3'-bis(4-(trifluoromethyl)phenyl)-[1,1'-binaphthalene]-2,2'-diol (S9a)}

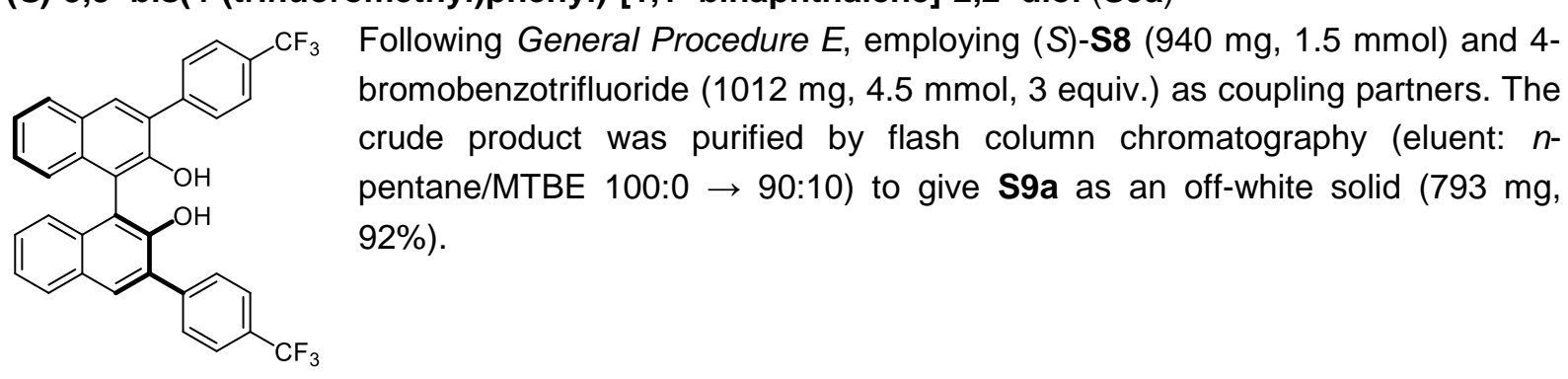

${ }^{1} \mathrm{H}$ NMR $\left(501 \mathrm{MHz}, \mathrm{CDCl}_{3}\right) \delta 8.06(\mathrm{~s}, 2 \mathrm{H}), 7.96(\mathrm{~d}, J=8.3 \mathrm{~Hz}, 2 \mathrm{H}), 7.87(\mathrm{~d}, J=8.0 \mathrm{~Hz}, 4 \mathrm{H}), 7.74(\mathrm{~d}, J$ $=7.9 \mathrm{~Hz}, 4 \mathrm{H}$ ), 7.44 (ddd, $J=8.2,6.8,1.3 \mathrm{~Hz}, 2 \mathrm{H}$ ), 7.37 (ddd, $J=8.2,6.8,1.3 \mathrm{~Hz}, 2 \mathrm{H}$ ), 7.23 (d, $J=8.4$ $\mathrm{Hz}, 2 \mathrm{H}), 5.31(\mathrm{~s}, 2 \mathrm{H})$.

${ }^{19} \mathrm{~F} \mathrm{NMR}\left(471 \mathrm{MHz}, \mathrm{CDCl}_{3}\right) \delta-62.49(\mathrm{~s}, 6 \mathrm{~F})$.

${ }^{13} \mathrm{C}$ NMR (126 MHz, CDCl $)$ ) $150.2(\mathrm{C}), 141.3(\mathrm{C}), 133.2(\mathrm{C}), 132.2(\mathrm{CH}), 130.1(\mathrm{CH}), 125.4$ (q, $J=$ $32.2 \mathrm{~Hz}, \mathrm{C}), 129.6(\mathrm{C}), 129.5(\mathrm{C}), 128.9(\mathrm{CH}), 128.2(\mathrm{CH}), 125.4$ (q, J = 3.8 Hz, CH), $125.0(\mathrm{CH})$, $124.2(\mathrm{CH}), 112.1(\mathrm{C})$. Spectroscopic data was consistent with the values reported in the literature. ${ }^{24}$ $\left[\alpha_{D}^{25}\right]=-65.3\left(c=0.44, \mathrm{CHCl}_{3}\right)$; literature (for $(R)$-enantiomer): $\left[\alpha_{D}^{23}\right]=+48.1\left(c=0.21, \mathrm{CHCl}_{3}\right) .{ }^{24}$

(S)-3,3'-bis(3-bromo-4-(trifluoromethyl)phenyl)-[1,1'-binaphthalene]-2,2'-diol (S9b)<smiles>Oc1c(-c2ccc(C(F)(F)F)c(Br)c2)cc2ccccc2c1-c1c(O)c(-c2ccc(C(F)(F)F)c(Br)c2)cc2ccccc12</smiles>
Following General Procedure E, employing (S)-S8 (626 mg, $1 \mathrm{mmol}$ ) and 2bromo-4-iodo-1-(trifluoromethyl)benzene (S5, $633 \mathrm{mg}, 2.3 \mathrm{mmol}, 2.3$ equiv.) as coupling partners. The crude product was purified by flash column chromatography (eluent: $n$-pentane/ $\mathrm{CH}_{2} \mathrm{Cl}_{2}$ 100:0 $\rightarrow$ 70:30) to give S9b as a white foamy solid (467 mg, 64\%).

${ }^{1} \mathrm{H}$ NMR (501 MHz, $\mathrm{CD}_{2} \mathrm{Cl}_{2}$ ) $\delta 8.16(\mathrm{~s}, 2 \mathrm{H}), 8.10(\mathrm{~s}, 2 \mathrm{H}), 8.00$ (d, $\left.J=7.3 \mathrm{~Hz}, 2 \mathrm{H}\right), 7.84$ (d, $J=8.2 \mathrm{~Hz}$, 2H), 7.81 (d, $J=8.4 \mathrm{~Hz}, 2 \mathrm{H}$ ), 7.46 (ddd, $J=8.1,6.8,1.2 \mathrm{~Hz}, 2 \mathrm{H}$ ), 7.39 (ddd, $J=8.2,6.8,1.3 \mathrm{~Hz}, 2 \mathrm{H}$ ), $7.19(\mathrm{~d}, J=8.4 \mathrm{~Hz}, 2 \mathrm{H}), 5.44(\mathrm{~s}, 2 \mathrm{H})$.

${ }^{19} \mathrm{~F}$ NMR $\left(471 \mathrm{MHz}, \mathrm{CD}_{2} \mathrm{Cl}_{2}\right) \delta-62.59(\mathrm{~s}, 6 \mathrm{~F})$.

${ }^{13} \mathrm{C} \mathrm{NMR}\left(126 \mathrm{MHz}, \mathrm{CD}_{2} \mathrm{Cl}_{2}\right) \delta 150.5(\mathrm{C}), 143.4(\mathrm{C}), 136.3(\mathrm{CH}), 133.8(\mathrm{C}), 132.7(\mathrm{CH}), 129.9(\mathrm{C})$, $129.2(\mathrm{CH}), 129.00(\mathrm{C}), 128.97(\mathrm{CH}), 128.7(\mathrm{CH}), 128.2(\mathrm{C}), 128.1$ (q, $J=5.4 \mathrm{~Hz}, \mathrm{CH}), 125.3(\mathrm{CH})$, $124.4(\mathrm{CH}), 123.6$ (d, $J=272.9 \mathrm{~Hz}, \mathrm{C}), 120.0(\mathrm{C}), 112.4(\mathrm{C})$.

ESI-HRMS: calculated for $\mathrm{C}_{34} \mathrm{H}_{17} \mathrm{Br}_{2} \mathrm{~F}_{6} \mathrm{O}_{2}{ }^{-}\left([\mathrm{M}-\mathrm{H}]^{-}\right): 728.9505$, found: 728.9514 .

$\left[\alpha_{D}^{25}\right]=-37.6\left(c=0.20, \mathrm{CHCl}_{3}\right)$. 


\section{(S)-3,3'-bis(4-(perfluoropropyl)phenyl)-[1,1'-binaphthalene]-2,2'-diol (S9c)}<smiles>Oc1c(-c2ccc(C(F)(F)F)cc2)cc2ccccc2c1-c1c(O)c(-c2ccc(C(F)(F)C(F)(F)F)cc2)cc2ccccc12</smiles>

Following General Procedure E, employing (S)-S8 (626 mg, $1 \mathrm{mmol}$ ) and 4(perfluoropropyl)phenyl trifluoromethanesulfonate $(\mathbf{S} 7,633 \mathrm{mg}, 2.3 \mathrm{mmol}$, 2.3 equiv.) as coupling partners. The crude product was purified by flash column chromatography (eluent: $n$-pentane/ $\mathrm{CH}_{2} \mathrm{Cl}_{2}$ 100:0 $\rightarrow 70: 30$ ) to give S9c as a white foamy solid (467 $\mathrm{mg}, 64 \%)$.

${ }^{1} \mathrm{H}$ NMR $\left(501 \mathrm{MHz}, \mathrm{CD}_{2} \mathrm{Cl}_{2}\right) \delta 8.11(\mathrm{~s}, 2 \mathrm{H}), 7.99(\mathrm{~d}, J=8.2 \mathrm{~Hz}, 2 \mathrm{H}), 7.93(\mathrm{~d}, J=8.5 \mathrm{~Hz}, 4 \mathrm{H}), 7.72(\mathrm{~d}, J$ $=8.3 \mathrm{~Hz}, 4 \mathrm{H}$ ), 7.45 (ddd, $J=8.0,6.8,1.2 \mathrm{~Hz}, 2 \mathrm{H}$ ), 7.37 (ddd, $J=8.2,6.8,1.3 \mathrm{~Hz}, 2 \mathrm{H}$ ), 7.21 (d, $J=8.5$ $\mathrm{Hz}, 2 \mathrm{H}), 5.45(\mathrm{~s}, 2 \mathrm{H})$.

${ }^{19} \mathrm{~F} \mathrm{NMR}\left(471 \mathrm{MHz}, \mathrm{CD}_{2} \mathrm{Cl}_{2}\right.$ ) $\delta-80.32$ (t, $\left.J=10.0 \mathrm{~Hz}, 6 \mathrm{~F}\right),-111.71$ (q, $\left.J=9.8 \mathrm{~Hz}, 4 \mathrm{~F}\right),-126.50$ (s, 4F). ${ }^{13} \mathrm{C}$ NMR $\left(126 \mathrm{MHz}, \mathrm{CD}_{2} \mathrm{Cl}_{2}\right.$ ) $\delta 150.6,142.1,133.7,132.6,130.4,130.0,129.7,129.1,128.3,128.0$, $127.1(\mathrm{t}, J=6.4 \mathrm{~Hz}), 125.1,124.4,112.5$.

ESI-HRMS: calculated for $\mathrm{C}_{38} \mathrm{H}_{19} \mathrm{~F}_{14} \mathrm{O}_{2}{ }^{-}\left([\mathrm{M}-\mathrm{H}]^{-}\right)$: 773.1167 , found: 773.1172 . $\left[\alpha_{D}^{25}\right]=-37.1\left(c=0.18, \mathrm{CHCl}_{3}\right)$.

(S)-6,6"'-bis(perfluoropropan-2-yl)-[2,2':4',1":3",2"'-quaternaphthalene]-2",3'-diol (S9d)<smiles>Oc1c(-c2ccc3cc(C(F)(F)C(F)(F)F)ccc3c2)cc2ccccc2c1-c1c(O)c(-c2ccc3cc(C(F)(F)C(F)(F)F)ccc3c2)cc2cc(C(F)(F)C(F)(F)F)ccc12</smiles>
Following General Procedure E, employing (S)-S8 (313 mg, $0.5 \mathrm{mmol})$ and 6-(perfluoropropan-2-yl)naphthalen-2-yl trifluoromethanesulfonate (S6, $489 \mathrm{mg}, 1.1 \mathrm{mmol}, 2.2$ equiv.) as coupling partners. The crude product was purified by flash column chromatography (eluent: $n$ pentane/MTBE 100:0 $\rightarrow$ 90:10) to give S9d as a light yellow solid (410 mg, 94\%).

${ }^{1} \mathrm{H}$ NMR $\left(501 \mathrm{MHz}, \mathrm{CD}_{2} \mathrm{Cl}_{2}\right) \delta 8.31(\mathrm{~s}, 2 \mathrm{H}), 8.24(\mathrm{~s}, 2 \mathrm{H}), 8.19(\mathrm{~s}, 2 \mathrm{H}), 7.71(\mathrm{~d}, J=8.8 \mathrm{~Hz}, 2 \mathrm{H}), 7.46$ (ddd, $J=8.1,6.8,1.2 \mathrm{~Hz}, 2 \mathrm{H}$ ), 7.39 (ddd, $J=8.3,6.8,1.4 \mathrm{~Hz}, 2 \mathrm{H}$ ), 7.28 (dd, $J=8.4,1.1 \mathrm{~Hz}, 2 \mathrm{H}$ ), 5.53 $(\mathrm{s}, 2 \mathrm{H})$.

${ }^{19} \mathrm{~F} \mathrm{NMR}\left(471 \mathrm{MHz}, \mathrm{CD}_{2} \mathrm{Cl}_{2}\right) \delta-75.72(\mathrm{~d}, J=7.3 \mathrm{~Hz}),-181.95$ (hept, $J=7.5 \mathrm{~Hz}$ ).

${ }^{13} \mathrm{C}$ NMR $\left(126 \mathrm{MHz}, \mathrm{CD}_{2} \mathrm{Cl}_{2}\right.$ ) $\delta$ 150.9, 138.0, 134.6, 133.7, $132.5(\mathrm{CH}), 132.2(\mathrm{~d}, J=2.1 \mathrm{~Hz}), 130.5$, 130.1, $129.68(\mathrm{CH}), 129.66(\mathrm{CH}), 129.1(\mathrm{CH}), 129.0(\mathrm{CH}), 128.6(\mathrm{CH}), 128.1(\mathrm{CH}), 126.7(\mathrm{~d}, J=11.8$ $\mathrm{Hz}, \mathrm{CH}), 125.0(\mathrm{CH}), 124.5(\mathrm{CH}), 124.4,122.5,122.3(\mathrm{q}, J=9.4 \mathrm{~Hz}, \mathrm{CH}), 120.1$ (d, J = 28.6 Hz), 112.8 .

ESI-HRMS: calculated for $\mathrm{C}_{46} \mathrm{H}_{23} \mathrm{O}_{2} \mathrm{~F}_{14^{-}}\left([\mathrm{M}-\mathrm{H}]^{-}\right)$: 873.1480, found: 873.1480 .

$\left[\alpha_{D}^{25}\right]=+36.3\left(c=0.25, \mathrm{CHCl}_{3}\right)$. 


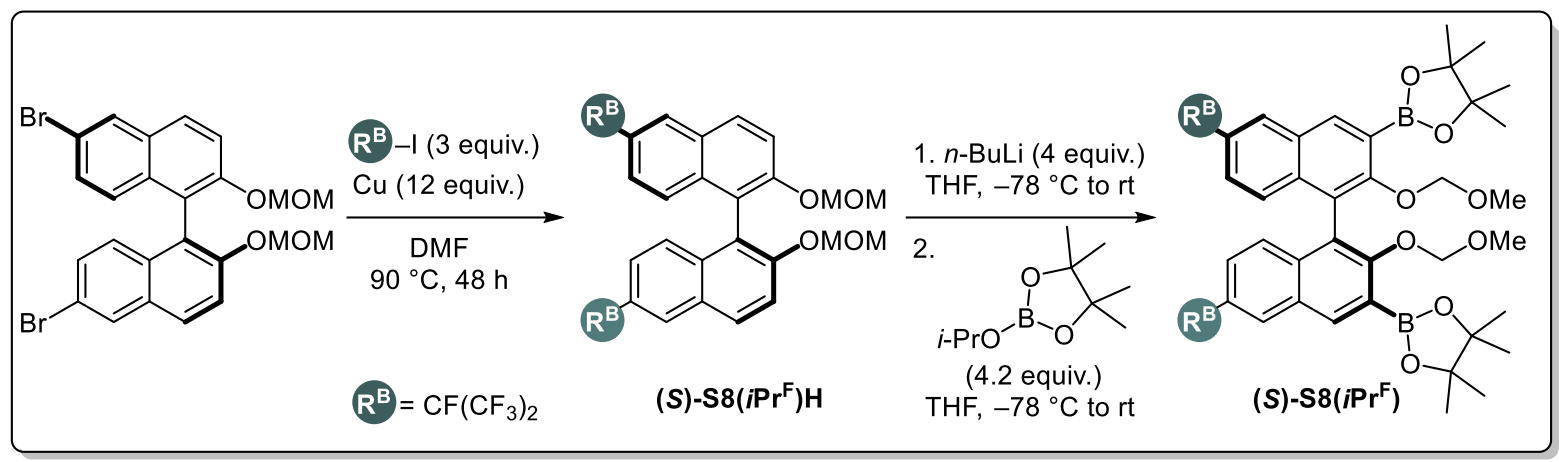

(S)-2,2'-bis(methoxymethoxy)-6,6'-bis(perfluoropropan-2-yl)-1,1'-binaphthalene (S8(iPrF)H)<smiles>COc1ccc2cc(C(F)(F)F)ccc2c1-c1c(OC)ccc2cc(C(F)(F)C(F)(F)F)ccc12</smiles>

Activation of copper: Copper powder $(5 \mathrm{~g})$ was stirred for $30 \mathrm{~min}$ in aq. $\mathrm{HCl} 1$ $\mathrm{M}(100 \mathrm{~mL})$, and then filtered with a Büchner funnel, washing with distilled water $(2 \times 100 \mathrm{~mL})$, ethanol $(1 \times 50 \mathrm{~mL})$, acetone $(1 \times 50 \mathrm{~mL})$ and $\mathrm{Et}_{2} \mathrm{O}(1 \times$ $50 \mathrm{~mL}$ ). The obtained solid is transferred to a flask and dried under vacuum (10-3 mbar) overnight.

Ullmann coupling: An oven-dried Schlenk flask, equipped with a magnetic stir bar, was charged under Ar with freshly activated copper powder (1.70 g, $26.7 \mathrm{mmol}, 6$ equiv.), DMF (10 mL), (S)-6,6'-dibromo-2,2'-bis(methoxymethoxy)-1,1'-binaphthalene ${ }^{25}$ (1.19 g, $2.23 \mathrm{mmol}$ ), and heptafluoro-2-iodopropane $(0.95 \mathrm{~mL}, 6.68 \mathrm{mmol}, 3$ equiv.). The mixture was degassed by bubbling Ar for $10 \mathrm{~min}$, then the flask was closed and the mixture was heated under stirring at $90{ }^{\circ} \mathrm{C}$ for $48 \mathrm{~h}$. After allowing to cool to rt and checking full conversion (TLC monitoring), the mixture was filtered through a pad of Celite $\AA$, washing with MTBE $(150 \mathrm{~mL})$. The filtrate was washed with distilled water $(2 \times 100 \mathrm{~mL})$ and brine $(1 \times 50 \mathrm{~mL})$, then dried over anhydrous $\mathrm{Na}_{2} \mathrm{SO}_{4}$, filtered and concentrated under reduced pressure. Purification by flash column chromatography on silica gel ( $n$ pentane/MTBE 90:10 $\mathrm{v} / \mathrm{V}$ ) afforded the corresponding product $\mathbf{S 8}(\mathbf{P P F}) \mathbf{H}$ as a yellow solid $(1.10 \mathrm{~g}, 70 \%$ yield).

${ }^{1} \mathrm{H}$ NMR (501 MHz, $\left.\mathrm{CD}_{2} \mathrm{Cl}_{2}\right) \delta 8.21(\mathrm{~s}, 2 \mathrm{H}), 8.10(\mathrm{~d}, J=9.1 \mathrm{~Hz}, 2 \mathrm{H}), 7.73(\mathrm{~d}, J=9.1 \mathrm{~Hz}, 2 \mathrm{H}), 7.40(\mathrm{~d}, J$ $=9.1 \mathrm{~Hz}, 2 \mathrm{H}), 7.25(\mathrm{~d}, J=9.1 \mathrm{~Hz}, 2 \mathrm{H}), 5.14(\mathrm{~d}, J=6.9 \mathrm{~Hz}, 2 \mathrm{H}), 5.09(\mathrm{~d}, J=7.0 \mathrm{~Hz}, 2 \mathrm{H}), 3.17(\mathrm{~s}, 6 \mathrm{H})$. ${ }^{19} \mathrm{~F}$ NMR $\left(471 \mathrm{MHz}, \mathrm{CD}_{2} \mathrm{Cl}_{2}\right) \delta-75.84$ (d, $\left.J=7.0 \mathrm{~Hz}, 12 \mathrm{~F}\right),-182.07$ (hept, $J=7.8,7.2 \mathrm{~Hz}, 2 \mathrm{~F}$ ).

${ }^{13} \mathrm{C} \mathrm{NMR}\left(126 \mathrm{MHz}, \mathrm{CD}_{2} \mathrm{Cl}_{2}\right.$ ) $\delta 154.8(\mathrm{C}), 135.1(\mathrm{C}), 131.0(\mathrm{CH}), 129.12(\mathrm{C}), 129.11(\mathrm{C}), 127.0$ (d, J = $11.8 \mathrm{~Hz}, \mathrm{CH}$ ), 126.6 (d, $J=2.3 \mathrm{~Hz}, \mathrm{CH}), 122.5$ (d, $J=9.8 \mathrm{~Hz}, \mathrm{CH}), 122.3$ (d, $J=20.6 \mathrm{~Hz}, \mathrm{C}), 120.2$ (C), $119.9(\mathrm{C}), 118.2(\mathrm{CH}), 95.3\left(\mathrm{CH}_{2}\right), 56.2\left(\mathrm{CH}_{3}\right)$.

ESI-HRMS: calculated for $\mathrm{C}_{30} \mathrm{H}_{20} \mathrm{~F}_{14} \mathrm{O}_{4} \mathrm{Na}^{+}\left([\mathrm{M}+\mathrm{Na}]^{+}\right)$: 733.1030, found: 733.1034. $\left[\alpha_{D}^{25}\right]=-41.2\left(c=0.61, \mathrm{CH}_{2} \mathrm{Cl}_{2}\right)$.

\section{(S)-2,2'-(2,2'-bis(methoxymethoxy)-6,6'-bis(perfluoropropan-2-yl)-[1,1'-binaphthalene]-3,3'-} diyl)bis(4,4,5,5-tetramethyl-1,3,2-dioxaborolane) $\left(\mathrm{S} 8\left(\operatorname{PrF}^{\mathrm{F}}\right)\right)$

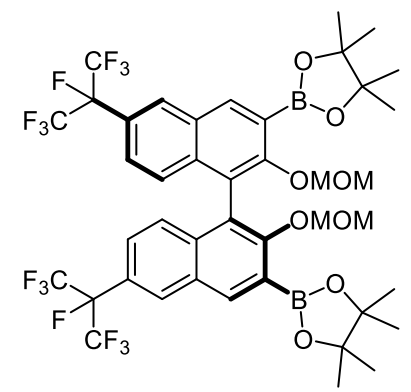

An oven-dried RB flask, equipped with a magnetic stir bar, was charged under Ar with $\mathbf{S} 8(\mathbb{P r F}) \mathbf{H}(1.00 \mathrm{~g}, 1.41 \mathrm{mmol})$ and THF $(15 \mathrm{~mL})$. After cooling the mixture at $-78^{\circ} \mathrm{C}, n$-BuLi $(2.5 \mathrm{M}$ in hexanes, $2.3 \mathrm{~mL}, 5.8 \mathrm{mmol}$, 4 equiv.) was added dropwise over $5 \mathrm{~min}$. After the addition was completed, the mixture was stirred for $30 \mathrm{~min}$ at $-78^{\circ} \mathrm{C}$ and then for further $3 \mathrm{~h}$ at rt. The mixture was cooled again to $-78^{\circ} \mathrm{C}$ and 2-isopropoxy4,4,5,5-tetramethyl-1,3,2-dioxaborolane (1.2 $\mathrm{mL}, 5.91 \mathrm{mmol}, 4.2$ equiv.) was added dropwise over $2 \mathrm{~min}$, followed by stirring at $\mathrm{rt}$ for $16 \mathrm{~h}$ (overnight). The reaction was quenched at $0{ }^{\circ} \mathrm{C}$ by adding $1 \mathrm{~mL} \mathrm{MeOH}$, followed by dilution with 100 $\mathrm{mL}$ distilled water. The mixture was extracted with MTBE $(3 \times 50 \mathrm{~mL})$, and the combined organic 
phase was washed with brine $(1 \times 30 \mathrm{~mL})$, dried over anhydrous $\mathrm{Na}_{2} \mathrm{SO}_{4}$, filtered and concentrated under reduced pressure. Purification by recrystallization (with hexanes) afforded the corresponding bis-boronate $\mathbf{S 8}(\mathbf{P P F})$ as a yellow solid $(989 \mathrm{mg}, 73 \%)$.

${ }^{1} \mathrm{H}$ NMR $\left(501 \mathrm{MHz}, \mathrm{CD}_{2} \mathrm{Cl}_{2}\right) \delta 8.59(\mathrm{~s}, 2 \mathrm{H}), 8.26(\mathrm{~d}, J=2.2 \mathrm{~Hz}, 2 \mathrm{H}), 7.48(\mathrm{dd}, J=9.1,2.0 \mathrm{~Hz}, 2 \mathrm{H})$, $7.36(\mathrm{~d}, J=9.1 \mathrm{~Hz}, 2 \mathrm{H}), 4.92(\mathrm{~d}, J=6.4 \mathrm{~Hz}, 2 \mathrm{H}), 4.82(\mathrm{~d}, J=6.4 \mathrm{~Hz}, 2 \mathrm{H}), 2.21(\mathrm{~s}, 6 \mathrm{H}), 1.40(\mathrm{~s}, 24 \mathrm{H})$.

${ }^{11} \mathrm{~B}$ NMR $\left(161 \mathrm{MHz}, \mathrm{CD}_{2} \mathrm{Cl}_{2}\right) \delta 30.20$.

${ }^{19} \mathrm{~F} \mathrm{NMR}\left(471 \mathrm{MHz}, \mathrm{CD}_{2} \mathrm{Cl}_{2}\right.$ ) $\delta-75.86$ (d, $\left.J=7.2 \mathrm{~Hz}, 12 \mathrm{~F}\right),-182.12$ (hept, $\left.J=7.4 \mathrm{~Hz}, 2 \mathrm{~F}\right)$.

${ }^{13} \mathrm{C}$ NMR (126 MHz, $\mathrm{CD}_{2} \mathrm{Cl}_{2}$ ) $\delta 159.7(\mathrm{C}), 140.7(\mathrm{CH}), 136.9(\mathrm{C}), 131.2(\mathrm{C}), 129.7$ (d, J = 2.1 Hz, C), $127.9(\mathrm{~d}, J=2.3 \mathrm{~Hz}, \mathrm{CH}), 127.4(\mathrm{~d}, J=11.3 \mathrm{~Hz}, \mathrm{CH}), 127.1(\mathrm{C}), 125.4(\mathrm{C}), 124.7$ (C), 123.2 (d, $J=$ $10.4 \mathrm{~Hz}, \mathrm{CH}$ ), 123.1 (C), 123.0 (C), 122.3 (d, $J=28.1 \mathrm{~Hz}, \mathrm{C}), 120.0$ (d, $J=28.0 \mathrm{~Hz}, \mathrm{C}), 117.9$ (C), $100.8\left(\mathrm{CH}_{2}\right), 95.4(\mathrm{C}), 93.3(\mathrm{C}), 93.0(\mathrm{C}), 92.7(\mathrm{C}), 91.6(\mathrm{C}), 91.4(\mathrm{C}), 91.1(\mathrm{C}), 84.6(\mathrm{C}), 55.7\left(\mathrm{CH}_{3}\right)$, $25.08\left(\mathrm{CH}_{3}\right), 25.06\left(\mathrm{CH}_{3}\right)$.

ESI-HRMS: calculated for $\mathrm{C}_{42} \mathrm{H}_{42} \mathrm{~B}_{2} \mathrm{~F}_{14} \mathrm{O}_{8} \mathrm{Na}^{+}\left([\mathrm{M}+\mathrm{Na}]^{+}\right)$: 985.2734, found: 985.2738.

$\left[\alpha_{D}^{25}\right]=-31.6\left(c=0.72, \mathrm{CH}_{2} \mathrm{Cl}_{2}\right)$.

\section{(S)-6,6'-bis(perfluoropropan-2-yl)-3,3'-bis(4-(trifluoromethyl)phenyl)-[1,1'-binaphthalene]-2,2'-} diol (S9e)<smiles>Oc1c(-c2ccc(C(F)(F)F)cc2)cc2cc3cc(C(F)(F)F)ccc3c-2c(-c2ccc(C(F)(F)F)cc2)c1-c1ccc(C(F)(F)F)cc1</smiles>

Following General Procedure E, but using (S)-S8(iPrF) as modified BINOL-diboronate (150 mg, $0.15 \mathrm{mmol}, 1$ equiv.), and 4bromobenzotrifluoride ( $88 \mathrm{mg}, 0.39 \mathrm{mmol}, 2.5$ equiv.) as coupling partner. The crude product was purified by flash column chromatography (eluent: $n$-pentane/MTBE 100:0 $\rightarrow$ 95:5) to give S9e as a light yellow solid (132 mg, 93\%).

${ }^{1} \mathrm{H}$ NMR $\left(501 \mathrm{MHz}, \mathrm{CD}_{2} \mathrm{Cl}_{2}\right) \delta 8.30(\mathrm{~s}, 2 \mathrm{H}), 8.21(\mathrm{~s}, 2 \mathrm{H}), 7.90(\mathrm{~d}, J=8.1$ $\mathrm{Hz}, 4 \mathrm{H}), 7.80(\mathrm{~d}, J=8.1 \mathrm{~Hz}, 4 \mathrm{H}), 7.56(\mathrm{~d}, J=9.0 \mathrm{~Hz}, 2 \mathrm{H}), 7.37(\mathrm{~d}, J=$

$9.0 \mathrm{~Hz}, 2 \mathrm{H}), 5.60(\mathrm{~s}, 2 \mathrm{H})$.

${ }^{19} \mathrm{~F} \operatorname{NMR}\left(471 \mathrm{MHz}, \mathrm{CD}_{2} \mathrm{Cl}_{2}\right) \delta-62.90(\mathrm{~s}, 6 \mathrm{~F}),-75.79$ (h, $\left.J=6.4 \mathrm{~Hz}, 12 \mathrm{~F}\right),-182.09$ (hept, $J=6.9 \mathrm{~Hz}$, $2 \mathrm{~F})$.

${ }^{13} \mathrm{C} \mathrm{NMR}\left(126 \mathrm{MHz}, \mathrm{CD}_{2} \mathrm{Cl}_{2}\right) \delta 152.5(\mathrm{C}), 141.0(\mathrm{C}), 134.6(\mathrm{C}), 133.4(\mathrm{CH}), 131.5(\mathrm{C}), 130.5(\mathrm{CH})$, 130.4 (q, J = 32.5 Hz, C), 129.1 (d, $J=2.1 \mathrm{~Hz}, \mathrm{C}$ ), 128.0 (C), 127.7 (d, $J=11.8 \mathrm{~Hz}, \mathrm{CH}$ ), 125.9 (q, $J=$ 3.7 Hz, CH), 125.6 (d, J = 2.2 Hz, CH), 124.1 (d, J=9.5 Hz, CH), 123.7 (C), 123.2 (d, $J=20.4 \mathrm{~Hz}, \mathrm{C}$ ), 122.3 (d, $J=27.6 \mathrm{~Hz}, \mathrm{C}$ ), 121.5 (C), 120.0 (d, $J=28.1 \mathrm{~Hz}, \mathrm{C}$ ), 112.2 (C), 93.0 (hept, $J=33.1 \mathrm{~Hz}, \mathrm{C}$ ), 91.4 (hept, $J=33.2 \mathrm{~Hz}, \mathrm{C}$ ).

ESI-HRMS: calculated for $\mathrm{C}_{40} \mathrm{H}_{17} \mathrm{~F}_{20} \mathrm{O}_{2}{ }^{-}\left([\mathrm{M}-\mathrm{H}]^{-}\right)$: 909.0915 , found: 909.0927 .

$\left[\alpha_{D}^{25}\right]=-61.4\left(c=0.17, \mathrm{CHCl}_{3}\right)$. 


\subsection{Synthesis of imino-imidodiphosphates}

\section{General Procedure F: One-pot synthesis of ilDPs}

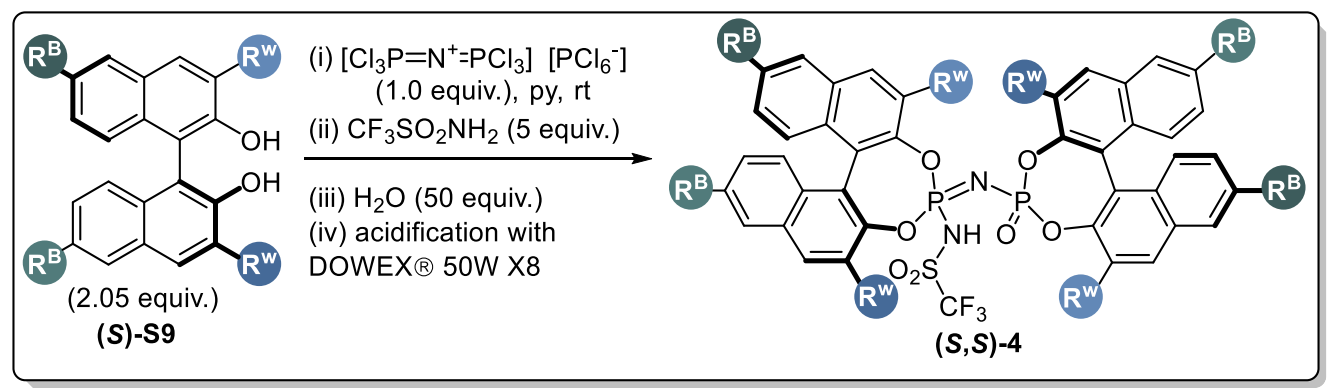

This is a slight adaptation from a reported procedure by our group. ${ }^{26}$

\section{A- Synthesis and purification}

An oven-dried Schlenk flask was charged with hexachlorobisphosphazonium hexachlorophosphate ${ }^{26}$ (107 mg, 0.20 mmol, 1 equiv.) and the corresponding (S)-BINOL (S9, 0.42 mmol, 2.1 equiv.) under Ar. After drying the mixture under vacuum $\left(10^{-3} \mathrm{mbar}\right)$ for $30 \mathrm{~min}$ at $\mathrm{rt}$ and switching back to $\mathrm{Ar}$ atmosphere, pyridine $(2 \mathrm{~mL})$ was added in one portion and the mixture was vigorously stirred for $2 \mathrm{~h}$. Trifluoromethanesulfonamide ( $149 \mathrm{mg}, 1.0 \mathrm{mmol}, 5$ equiv.) was added in one portion and the mixture was further stirred at rt overnight, followed by the addition of distilled water $(0.18 \mathrm{~mL}, 10 \mathrm{mmol}, 50$ equiv.) and additional stirring for $2 \mathrm{~h}$. The reaction was stopped by diluting the mixture with $\mathrm{CH}_{2} \mathrm{Cl}_{2}(10$ $\mathrm{mL}$ ) and adding aq. $\mathrm{HCl} 3 \mathrm{M}(10 \mathrm{~mL})$; after stirring for $30 \mathrm{~min}$, the aqueous phase was extracted with $\mathrm{CH}_{2} \mathrm{Cl}_{2}(3 \times 30 \mathrm{~mL})$. The combined organic phases were washed with distilled water $(1 \times 30 \mathrm{~mL})$ and brine $(1 \times 30 \mathrm{~mL})$, then dried over anhydrous $\mathrm{Na}_{2} \mathrm{SO}_{4}$, filtered and concentrated under reduced pressure. Purification by flash column chromatography on silica gel ( $n$-hexane/EtOAc mixtures) afforded the corresponding ilDPs as salts.

\section{B- Acidification}

DOWEX® 50W X8 (approx. $5 \mathrm{~g}$ ) was suspended in aq. $\mathrm{H}_{2} \mathrm{SO}_{4} 0.5 \mathrm{M}$, transferred to a glass column with stopcock, and washed thoroughly with more acid (approximately 20 times the volume of the DOWEX pad) until the eluate was colorless. Then, the resin pad was washed with distilled water (until the eluate is neutral to $\mathrm{pH}$ indicator paper), ethanol and $\mathrm{Et}_{2} \mathrm{O}$, in that order. For each of these washing operations, 10-20 times of pad volume was used as volume of the washing agent.

The purified ilDP salt (after column, dissolved in 5-10 mL Et ${ }_{2} \mathrm{O}$ ) was added to the DOWEX pad (packed in $\mathrm{Et}_{2} \mathrm{O}$ ) and the eluate was collected in test tubes (adding more $\mathrm{Et}_{2} \mathrm{O}$ to not let the DOWEX pad run dry). The eluted fractions were re-added to the DOWEX pad to ensure full acidification: the collection and re-acidification was done $3 \mathrm{x}$ in total. After washing the DOWEX pad with $\mathrm{Et}_{2} \mathrm{O}$, the eluate was concentrated under reduced pressure to give a light yellow solid. This solid was redissolved in a small amount of $\mathrm{Et}_{2} \mathrm{O}(1-2 \mathrm{~mL})$ and treated with $n$-pentane $(20 \mathrm{~mL})$. After removing the solvents under reduced pressure, the obtained solid was further freeze-dried with liquid $\mathrm{N}_{2}(3 \mathrm{x})$ and dried overnight under high-vacuum, affording thus the acidified imino-imidodiphosphate 4. 
1,1,1-trifluoro-N-((11bS)-4-(((11bS)-4-oxido-2,6-bis(4-(trifluoromethyl)phenyl)dinaphtho[2,1$\left.d: 1^{\prime}, 2^{\prime}-f\right][1,3,2]$ dioxaphosphepin-4-yl)imino)-2,6-bis(4-(trifluoromethyl)phenyl)-4 $\lambda^{5}$ dinaphtho[2,1-d:1',2'-f][1,3,2]dioxaphosphepin-4-yl)methanesulfonamide (iDP 4b)

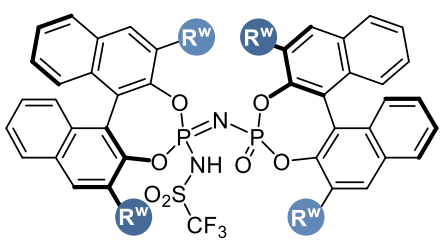

Following General Procedure F, employing (S)-BINOL S9a $(603 \mathrm{mg}$, $1.0 \mathrm{mmol}$ ) as starting material. The crude product was purified by flash column chromatography (eluent: $n$-pentane/ $\mathrm{CH}_{2} \mathrm{Cl}_{2} 100: 0 \rightarrow 70: 30$ ) and acidified with DOWEX® $50 \mathrm{~W} \times 8$, to give $\mathbf{4 b}$ as an off-white solid (530 $\mathrm{mg}, 77 \%)$.

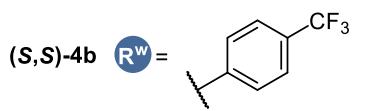

${ }^{1} \mathrm{H}$ NMR (501 MHz, $\left.\mathrm{CD}_{2} \mathrm{Cl}_{2}\right) \delta 8.22(\mathrm{~d}, J=8.2 \mathrm{~Hz}, 2 \mathrm{H}), 8.12(\mathrm{~d}, J=17.0 \mathrm{~Hz}, 2 \mathrm{H}), 8.07$ (dd, $J=8.3,5.3$ $\mathrm{Hz}, 2 \mathrm{H}$ ), 7.84 (dddd, $J=12.5,8.1,5.8,2.2 \mathrm{~Hz}, 2 \mathrm{H}), 7.72-7.57(\mathrm{~m}, 12 \mathrm{H}), 7.52-7.39(\mathrm{~m}, 8 \mathrm{H}), 7.03$ (d, $J=8.1 \mathrm{~Hz}, 2 \mathrm{H}), 6.98(\mathrm{~d}, J=8.1 \mathrm{~Hz}, 2 \mathrm{H}), 6.78(\mathrm{~d}, J=8.0 \mathrm{~Hz}, 2 \mathrm{H}), 6.72(\mathrm{~d}, J=8.0 \mathrm{~Hz}, 2 \mathrm{H})$.

${ }^{19} \mathrm{~F} \mathrm{NMR}\left(471 \mathrm{MHz}, \mathrm{CD}_{2} \mathrm{Cl}_{2}\right) \delta-62.58(\mathrm{~s}, 3 \mathrm{~F}),-62.73(\mathrm{~s}, 3 \mathrm{~F}),-62.77(\mathrm{~s}, 3 \mathrm{~F}),-63.16(\mathrm{~s}, 3 \mathrm{~F}),-80.03(\mathrm{~s}$, $3 F)$.

${ }^{31} \mathrm{P}$ NMR $\left(203 \mathrm{MHz}, \mathrm{CD}_{2} \mathrm{Cl}_{2}\right) \delta-2.35(\mathrm{~d}, J=111.5 \mathrm{~Hz}),-7.81(\mathrm{~d}, J=111.4 \mathrm{~Hz})$.

${ }^{13} \mathrm{C}$ NMR $\left(126 \mathrm{MHz}, \mathrm{CD}_{2} \mathrm{Cl}_{2}\right) \delta 144.3,144.2,143.9,143.8,143.7,140.4,140.1,140.0,132.94,132.87$, 132.8, $132.7(\mathrm{CH}), 132.5,132.33,132.27,132.1(\mathrm{CH}), 131.6(\mathrm{CH}), 131.1(\mathrm{CH}), 130.8(\mathrm{CH}), 130.3$, $130.2(\mathrm{CH}), 130.1(\mathrm{CH}), 130.0(\mathrm{CH}), 129.8,129.6,129.4(\mathrm{CH}), 129.3(\mathrm{CH}), 129.2(\mathrm{CH}), 128.5(\mathrm{CH})$, $128.2(\mathrm{CH}), 127.9(\mathrm{CH}), 127.8(\mathrm{CH}), 127.7(\mathrm{CH}), 127.5(\mathrm{CH}), 127.42(\mathrm{CH}), 127.36(\mathrm{CH}), 127.22(\mathrm{CH})$, $127.19(\mathrm{CH}), 127.1(\mathrm{CH}), 127.0(\mathrm{CH}), 125.84(\mathrm{CH}), 125.81(\mathrm{CH}), 125.11(\mathrm{CH}), 125.07(\mathrm{CH}), 125.00$ $(\mathrm{CH}), 124.97(\mathrm{CH}), 124.73(\mathrm{CH}), 124.69(\mathrm{CH}), 124.0,123.94,123.91,122.7,122.5$.

ESI-HRMS: calculated for $\mathrm{C}_{69} \mathrm{H}_{36} \mathrm{~F}_{15} \mathrm{~N}_{2} \mathrm{O}_{7} \mathrm{P}_{2} \mathrm{~S}^{-}\left([\mathrm{M}-\mathrm{H}]^{-}\right)$: 1383.1485 , found: 1383.1483 .

$\left[\alpha_{D}^{25}\right]=+314.0\left(c=0.23, \mathrm{CHCl}_{3}\right)$.

\section{1,1,1-trifluoro-N-((11bS)-4-(((11bS)-4-oxido-2,6-bis(3-bromo-4-}

(trifluoromethyl)phenyl)dinaphtho[2,1-d:1',2'-f][1,3,2]dioxaphosphepin-4-yl)imino)-2,6-bis(3bromo-4-(trifluoromethyl)phenyl)-4 $\lambda^{5}$-dinaphtho[2,1-d:1',2'-f][1,3,2]dioxaphosphepin-4yl)methanesulfonamide (iIDP 4c)

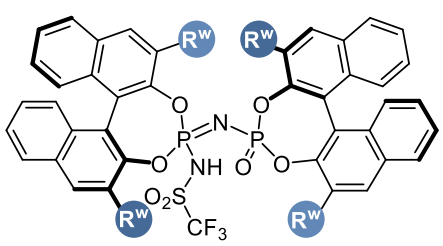

$(s, s)-4 c \quad R^{w}=C_{B r}^{C F_{3}}$

Following General Procedure F, employing (S)-BINOL S9b (384 mg, $0.52 \mathrm{mmol}$ ) as starting material. The crude product was purified by flash column chromatography (eluent: $n$-pentane $/ \mathrm{CH}_{2} \mathrm{Cl}_{2}$ 100:0 $\rightarrow$ 70:30) and acidified with DOWEX® $50 \mathrm{~W} X 8$, to give $4 \mathrm{c}$ as an off-white solid (286 mg, 67\%).

${ }^{1} \mathrm{H}$ NMR $\left(501 \mathrm{MHz}, \mathrm{CD}_{2} \mathrm{Cl}_{2}\right) \delta 8.22(\mathrm{~s}, 1 \mathrm{H}), 8.15(\mathrm{~s}, 1 \mathrm{H}), 8.09$ (t, $\left.J=6.7 \mathrm{~Hz}, 4 \mathrm{H}\right), 7.86$ (dd, $J=13.5,6.0$ $\mathrm{Hz}, 3 \mathrm{H}), 7.80(\mathrm{~d}, J=8.6 \mathrm{~Hz}, 1 \mathrm{H}), 7.76(\mathrm{~d}, J=8.5 \mathrm{~Hz}, 1 \mathrm{H}), 7.73-7.64(\mathrm{~m}, 5 \mathrm{H}), 7.61(\mathrm{~d}, J=7.3 \mathrm{~Hz}$, 2H), $7.48-7.28(\mathrm{~m}, 9 \mathrm{H}), 7.25(\mathrm{~s}, 1 \mathrm{H}), 6.99(\mathrm{t}, J=9.0 \mathrm{~Hz}, 2 \mathrm{H}), 6.69(\mathrm{~d}, J=8.2 \mathrm{~Hz}, 1 \mathrm{H}), 6.59(\mathrm{~d}, J=$ $8.2 \mathrm{~Hz}, 1 \mathrm{H})$.

${ }_{19}^{19} \mathrm{FMR}\left(471 \mathrm{MHz}, \mathrm{CD}_{2} \mathrm{Cl}_{2}\right) \delta-62.24(\mathrm{~s}, 3 \mathrm{~F}),-62.51(\mathrm{~s}, 3 \mathrm{~F}),-62.64(\mathrm{~s}, 3 \mathrm{~F}),-63.04(\mathrm{~s}, 3 \mathrm{~F}),-80.13(\mathrm{~s}$, $3 \mathrm{~F})$.

${ }^{31} \mathrm{P}$ NMR $\left(203 \mathrm{MHz}, \mathrm{CD}_{2} \mathrm{Cl}_{2}\right) \delta-3.53(\mathrm{~d}, J=111.3 \mathrm{~Hz}),-7.76(\mathrm{~d}, J=111.3 \mathrm{~Hz})$.

${ }^{13} \mathrm{C}$ NMR $\left(126 \mathrm{MHz}, \mathrm{CD}_{2} \mathrm{Cl}_{2}\right) \delta 144.1,144.0,143.9,143.7,143.6,142.7,141.7,141.6,141.5,141.4$, 136.2, 135.6, 135.5, 135.4, 132.8, 132.7, 132.61, 132.55, 132.4, 132.3, 132.2, 131.94, 131.90, 131.7, $131.4,131.3,131.00,130.97,130.78,130.76,129.7,129.6,129.5,129.4,129.2,128.9,128.8,128.64$, 
$128.55,128.2,128.0,127.92,127.88,127.84,127.81,127.75,127.5,127.4,127.3,127.2,127.0$, $124.7,124.52,124.46,124.3,124.02,124.00,123.84,123.82,122.8,122.7,122.5,122.4,122.1$, 120.2, 120.0, 119.8, 119.7.

ESI-HRMS: calculated for $\mathrm{C}_{69} \mathrm{H}_{32} \mathrm{Br}_{4} \mathrm{~F}_{15} \mathrm{~N}_{2} \mathrm{O}_{7} \mathrm{P}_{2} \mathrm{~S}^{-}\left([\mathrm{M}-\mathrm{H}]^{-}\right)$: 1694.7906 , found: 1694.7904 .

$\left[\alpha_{D}^{25}\right]=+279.3\left(c=0.23, \mathrm{CHCl}_{3}\right)$.

1,1,1-trifluoro-N-((11bS)-4-(((11bS)-4-oxido-2,6-bis(4-(perfluoropropyl)phenyl)dinaphtho[2,1$d: 1^{\prime}, 2$ '-f][1,3,2]dioxaphosphepin-4-yl)imino)-2,6-bis(4-(perfluoropropyl)phenyl)-4 $\lambda^{5}$ dinaphtho[2,1-d:1',2'-f][1,3,2]dioxaphosphepin-4-yl)methanesulfonamide (ilDP 4d)

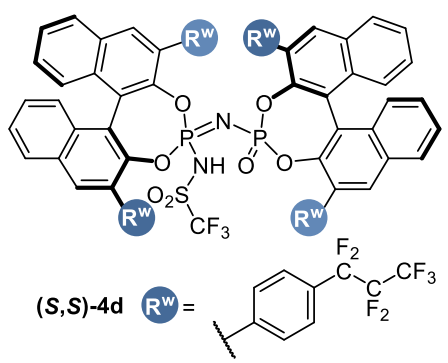

Following General Procedure F, employing (S)-BINOL S9c $(119 \mathrm{mg}$, $0.15 \mathrm{mmol}$ ) as starting material. The crude product was purified by flash column chromatography (eluent: $n$-pentane/ $\mathrm{CH}_{2} \mathrm{Cl}_{2}$ 100:0 $\rightarrow$ 70:30) and acidified with DOWEX® $50 \mathrm{~W}$ X8, to give $\mathbf{4 d}$ as an off-white solid (132 mg, 98\%).

${ }^{1} \mathrm{H}$ NMR (501 MHz, $\mathrm{CD}_{2} \mathrm{Cl}_{2}$ ) $\delta 8.22(\mathrm{dd}, J=8.3,5.6 \mathrm{~Hz}, 2 \mathrm{H}), 8.16(\mathrm{~s}, 1 \mathrm{H}), 8.12(\mathrm{~s}, 1 \mathrm{H}), 8.07$ (t, $J=8.6$ $\mathrm{Hz}, 2 \mathrm{H}), 7.88-7.79(\mathrm{~m}, 2 \mathrm{H}), 7.73(\mathrm{~s}, 1 \mathrm{H}), 7.67-7.57(\mathrm{~m}, 9 \mathrm{H}), 7.53(\mathrm{dt}, J=16.7,8.4 \mathrm{~Hz}, 5 \mathrm{H}), 7.46-$ $7.35(\mathrm{~m}, 5 \mathrm{H}), 7.00(\mathrm{~d}, J=8.1 \mathrm{~Hz}, 2 \mathrm{H}), 6.93(\mathrm{~d}, J=8.1 \mathrm{~Hz}, 2 \mathrm{H}), 6.78(\mathrm{~d}, J=8.2 \mathrm{~Hz}, 2 \mathrm{H}), 6.74(\mathrm{~d}, J=$ $8.2 \mathrm{~Hz}, 2 \mathrm{H})$.

${ }^{19} \mathrm{~F} \mathrm{NMR}\left(471 \mathrm{MHz}, \mathrm{CD}_{2} \mathrm{Cl}_{2}\right) \delta-79.96(\mathrm{~s}, 3 \mathrm{~F}),-80.35--80.54(\mathrm{~m}, 12 \mathrm{~F}),-110.29--112.62(\mathrm{~m}, 8 \mathrm{~F}),-$ $126.48--126.70(\mathrm{~m}, 8 \mathrm{~F})$.

${ }^{31} \mathrm{P}$ NMR $\left(203 \mathrm{MHz}, \mathrm{CD}_{2} \mathrm{Cl}_{2}\right) \delta-1.53(\mathrm{~d}, J=107.7 \mathrm{~Hz}),-7.14(\mathrm{~d}, J=108.4 \mathrm{~Hz})$.

${ }^{13} \mathrm{C}$ NMR $\left(151 \mathrm{MHz}, \mathrm{CD}_{2} \mathrm{Cl}_{2}\right) \delta 144.3,144.2,143.9,143.8,143.02,142.96,140.6,140.3,140.2$, $133.03,133.01,132.9,132.84,132.83,132.78,132.77,132.7,132.60,132.58,132.49,132.48,132.4$, $132.3,132.24,132.21,132.20,132.1,132.02,132.00,131.7,131.2,130.7,130.5,130.1,129.99$, $129.91,129.4$, 129.3, 129.2, 128.6, 128.4, 128.3, 128.1, 127.91, 127.86, 127.8, 127.7, 127.5, 127.40, $127.36,127.32,127.28,127.19,127.16,127.04,126.97,126.7,126.63,126.58,126.53,126.49$, $126.25,126.21,126.16,124.00,123.98,123.97,123.95,122.74,122.72,122.5,121.6,121.3,121.1$, $120.70,120.68,119.7,119.4,119.24,119.19,119.02,118.96,118.6,117.7,117.5,117.3,117.2$, $117.1,117.0,116.4,115.8,115.7,115.6,114.2,114.0,111.05,110.99,110.8,110.7,110.54,110.49$, 109.5, 109.3, 109.2, 109.05, 108.99, 108.8, 108.7, 108.5, 107.6, 107.5, 107.3, 107.2, 107.0.

ESI-HRMS: calculated for $\mathrm{C}_{77} \mathrm{H}_{36} \mathrm{~F}_{31} \mathrm{~N}_{2} \mathrm{O}_{7} \mathrm{P}_{2} \mathrm{~S}^{-}\left([\mathrm{M}-\mathrm{H}]^{-}\right)$: 1783.1229 , found: 1783.1237 .

$\left[\alpha_{D}^{25}\right]=+336.0\left(c=0.17, \mathrm{CHCl}_{3}\right)$.

1,1,1-trifluoro-N-((11bS)-4-(((11bS)-4-oxido-2,6-bis(6-(perfluoropropan-2-yl)naphthalen-2yl)dinaphtho[2,1-d:1',2'-f][1,3,2]dioxaphosphepin-4-yl)imino)-2,6-bis(6-(perfluoropropan-2yl)naphthalen-2-yl)-4 $\lambda^{5}$-dinaphtho[2,1-d:1',2'-f][1,3,2]dioxaphosphepin-4-yl)methanesulfonamide (iDP 4e)

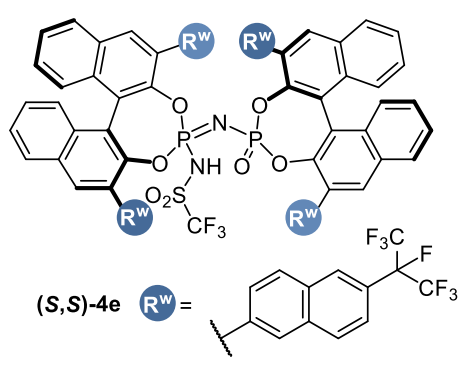

Following General Procedure F, employing (S)-BINOL S9d (350 mg, $0.40 \mathrm{mmol}$ ) as starting material. The crude product was purified by flash column chromatography (eluent: $i$-hexane/EtOAc 97:3 $\rightarrow$ 80:20) and acidified with DOWEX® $50 \mathrm{~W} \times 8$, to give $4 \mathrm{e}$ as an off-light yellow solid (261 mg, 69\%). 
${ }^{1} \mathrm{H}$ NMR $\left(501 \mathrm{MHz}, \mathrm{CD}_{2} \mathrm{Cl}_{2}\right) \delta 8.22(\mathrm{t}, J=7.0 \mathrm{~Hz}, 2 \mathrm{H}), 8.14-8.04(\mathrm{~m}, 5 \mathrm{H}), 7.98-7.87(\mathrm{~m}, 7 \mathrm{H}), 7.80$ (dt, $J=19.9,6.9 \mathrm{~Hz}, 2 \mathrm{H}), 7.71(\mathrm{dd}, J=8.9,5.3 \mathrm{~Hz}, 2 \mathrm{H}), 7.66-7.41(\mathrm{~m}, 18 \mathrm{H}), 7.39(\mathrm{~d}, J=8.7 \mathrm{~Hz}, 2 \mathrm{H})$, $7.36(\mathrm{~s}, 1 \mathrm{H}), 7.32(\mathrm{~s}, 1 \mathrm{H}), 6.85(\mathrm{dd}, J=8.7,1.9 \mathrm{~Hz}, 1 \mathrm{H}), 6.77(\mathrm{dd}, J=8.7,1.9 \mathrm{~Hz}, 1 \mathrm{H}), 6.42-6.33(\mathrm{~m}$, $2 \mathrm{H})$.

${ }^{19} \mathrm{~F}$ NMR (471 MHz, $\left.\mathrm{CD}_{2} \mathrm{Cl}_{2}\right) \delta-75.69--76.12$ (m, 24F), -80.84 (s, 3F), -181.95 (dhept, $J=21.5,7.1$ $\mathrm{Hz}, 2 \mathrm{~F}),-182.19$ (dhept, $J=21.9,7.1 \mathrm{~Hz}, 2 \mathrm{~F}$ ).

${ }^{31} \mathrm{P}$ NMR $\left(203 \mathrm{MHz}, \mathrm{CD}_{2} \mathrm{Cl}_{2}\right) \delta-4.69(\mathrm{~d}, J=120.7 \mathrm{~Hz}),-9.79(\mathrm{~d}, J=120.3 \mathrm{~Hz})$.

${ }^{13} \mathrm{C}$ NMR $\left(126 \mathrm{MHz}, \mathrm{CD}_{2} \mathrm{Cl}_{2}\right) \delta 144.4,144.1,136.3,136.1,135.8,134.3,134.2,134.1,134.0,133.6$, $133.5,133.18,133.15,133.1,133.0,132.6,132.5,132.43,132.38,132.3,132.12,132.06,132.0$, $131.9,131.7,131.6,129.8,129.72,129.67,129.6,129.5,129.4,129.3,129.2,129.1,128.8,128.7$, 128.6, 128.5, 128.4, 128.2, 128.1, 128.0, 127.84, 127.77, 127.64, 127.56, 127.5, 127.3, 127.1, 126.9, 126.4 , 126.2, 126.1, 124.8, 124.7, 124.6, 124.5, 124.4, 124.24, 124.17, 124.15, 124.1, 123.92, 123.88, 123.86, 122.72, 122.70, 122.4, 122.2, 121.9, 121.7, 121.6 .

ESI-HRMS: calculated for $\mathrm{C}_{93} \mathrm{H}_{44} \mathrm{~F}_{31} \mathrm{~N}_{2} \mathrm{O}_{7} \mathrm{P}_{2} \mathrm{~S}^{-}\left([\mathrm{M}-\mathrm{H}]^{-}\right)$: 1983.1855 , found: 1983.1863 .

$\left[\alpha_{D}^{25}\right]=+232.7\left(c=0.21, \mathrm{CHCl}_{3}\right)$.

\section{1,1,1-trifluoro-N-((11bS)-4-(((11bS)-4-oxido-9,14-bis(perfluoropropan-2-yl)-2,6-bis(4-}

(trifluoromethyl)phenyl)dinaphtho[2,1-d:1',2'-f][1,3,2]dioxaphosphepin-4-yl)imino)-9,14-

bis(perfluoropropan-2-yl)-2,6-bis(4-(trifluoromethyl)phenyl)-4 ${ }^{5}$-dinaphtho[2,1-d:1',2'-

$f][1,3,2]$ dioxaphosphepin-4-yl)methanesulfonamide (iDP 4b')
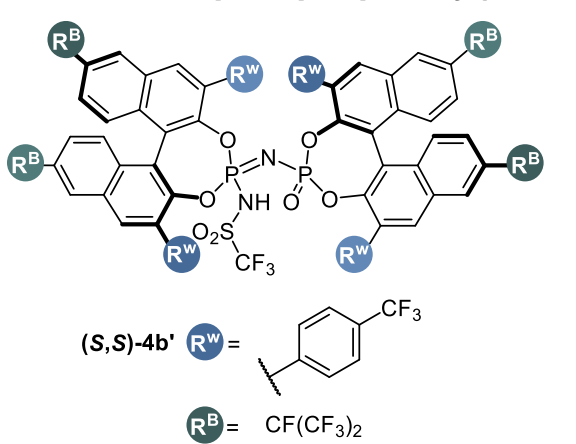

Following General Procedure F, employing (S)-BINOL S9e (326 $\mathrm{mg}, 0.35 \mathrm{mmol}$ ) as starting material. The crude product was purified by flash column chromatography (eluent: $n$ pentane $/ \mathrm{CH}_{2} \mathrm{Cl}_{2}$ 100:0 $\rightarrow$ 70:30) and acidified with DOWEX® $50 \mathrm{~W} \times 8$, to give $\mathbf{4 b}$ ' as a light yellow solid (326 $\mathrm{mg}, 91 \%$ ).

${ }^{1} \mathrm{H}$ NMR (501 MHz, $\left.\mathrm{CD}_{2} \mathrm{Cl}_{2}\right) \delta 8.55(\mathrm{~s}, 2 \mathrm{H}), 8.39(\mathrm{~s}, 2 \mathrm{H}), 8.25(\mathrm{~d}, J=7.0 \mathrm{~Hz}, 2 \mathrm{H}), 7.91-7.81(\mathrm{~m}, 4 \mathrm{H})$, $7.76(\mathrm{t}, J=9.0 \mathrm{~Hz}, 2 \mathrm{H}), 7.69-7.59(\mathrm{~m}, 7 \mathrm{H}), 7.56(\mathrm{~d}, J=9.2 \mathrm{~Hz}, 1 \mathrm{H}), 7.47(\mathrm{t}, J=8.8 \mathrm{~Hz}, 4 \mathrm{H}), 6.92-$ $6.83(\mathrm{~m}, 6 \mathrm{H}), 6.80(\mathrm{~d}, J=8.1 \mathrm{~Hz}, 2 \mathrm{H})$.

${ }^{19} \mathrm{~F}$ NMR $\left(471 \mathrm{MHz}, \mathrm{CD}_{2} \mathrm{Cl}_{2}\right) \delta-62.86(\mathrm{~s}, 6 \mathrm{~F}),-62.88(\mathrm{~s}, 3 \mathrm{~F}),-63.27$ (s, 3F), -75.52 - -75.59 (m, 16F), -75.66 (dp, $J=20.8,8.2 \mathrm{~Hz}, 8 \mathrm{~F}$ ), -79.94 (s, 3F), -182.13 (q, $J=6.9 \mathrm{~Hz}, 3 \mathrm{~F}$ ), -182.24 (p, $J=7.3$ $\mathrm{Hz}, 1 \mathrm{~F})$.

${ }^{31} \mathrm{P}$ NMR $\left(203 \mathrm{MHz}, \mathrm{CD}_{2} \mathrm{Cl}_{2}\right) \delta-1.75(\mathrm{~d}, J=101.0 \mathrm{~Hz}),-5.93(\mathrm{~d}, J=100.8 \mathrm{~Hz})$.

${ }^{13} \mathrm{C}$ NMR $\left(126 \mathrm{MHz}, \mathrm{CD}_{2} \mathrm{Cl}_{2}\right.$ ) $\delta 139.8,139.3,139.1,134.7,134.5,134.4,133.7,133.6,133.5,133.2$, $133.0,132.3,131.9,131.5,130.8,130.2,130.1,129.9,128.3,128.0,127.9,126.1,125.5,125.32$, $125.29,124.9,124.7,123.8,123.7,123.2,122.2,120.1$.

ESI-HRMS: calculated for $\mathrm{C}_{81} \mathrm{H}_{32} \mathrm{~F}_{43} \mathrm{~N}_{2} \mathrm{O}_{7} \mathrm{P}_{2} \mathrm{~S}^{-}\left([\mathrm{M}-\mathrm{H}]^{-}\right)$: 2055.0725 , found: 2055.0740 . $\left[\alpha_{D}^{25}\right]=+227.7\left(c=0.19, \mathrm{CHCl}_{3}\right)$. 


\section{Computational Studies}

\section{METHOD}

All calculations presented in this paper were carried out with a development version of the ORCA suite of programs base on version 4.2. ${ }^{27}$ Molecular geometries were optimized in the gas phase using the PBE functional28 in conjunction with the D3 version of Grimme's dispersion correction with BeckeJohnson damping function, ${ }^{29}$ using the resolution of identity approximation. The def2-SVP basis set was used for all atoms with matching auxiliary basis. ${ }^{30}$ Analytic frequency calculations were performed to verify the nature of all stationary points (minima and transition states) and to calculate free energies and enthalpies at $298 \mathrm{~K}$ by using the rigid-rotor harmonic oscillator (RRHO) approximation. Solvation effect has been accounted by using CPCM (cyclohexane) solvation model, ${ }^{31}$ as implemented in ORCA. An exhaustive manual conformational search was performed for possible catalyst-substrate orientations. Transition state (TS) structures were verified by the presence of a single imaginary vibrational frequency. Single-point energies are calculated at M06-2X/def2-TZVP32 level of theory. Distortion-Interaction analysis ${ }^{33}$ has been performed to determine the reason behind the stereoinduction. Molecular structures were generated using CYLview program ${ }^{34}$ and VMD. ${ }^{35}$

The main objectives of our computational analysis are the following:

-to understand the confinement effect ("cavity effect"): what causes such a different result during the deuterium-labelling experiments in the presence of achiral $p-\mathrm{TsOH}$, and chiral, enantiopure $(S, S)$-ilDP catalyst (Figures 3B and $3 \mathrm{C}$ of main text).

-to probe the origin of stereoselectivity.

\section{1. "Open" vs. "confined" acid: Understanding the "cavity effect"}

The results from the experiments with deuterium-containing reagents (Sections 6.1 and 6.2) showed a significant difference in the reaction pathways, when either $p-\mathrm{TsOH}$ or an iDP are utilized as catalysts. Inspired by the work of Kupova and coworkers, ${ }^{36}$ we conducted several computational calculations to study the addition of styrene (1a) to a formaldehyde-derived aldehydium ion ("truncated electrophile"), in the presence of the anion from the deprotonated catalyst (either $p$-TsOH or iDP, Scheme S-3). It is worth to mention that, in contrast to the study from Kupova, our proposed truncated electrophile does not contain a terminal $-\mathrm{OH}$ group, in order to describe more accurately the oligomeric structure of paraformaldehyde, as well as to avoid the participation of hydrogen-bond interactions in the calculations.

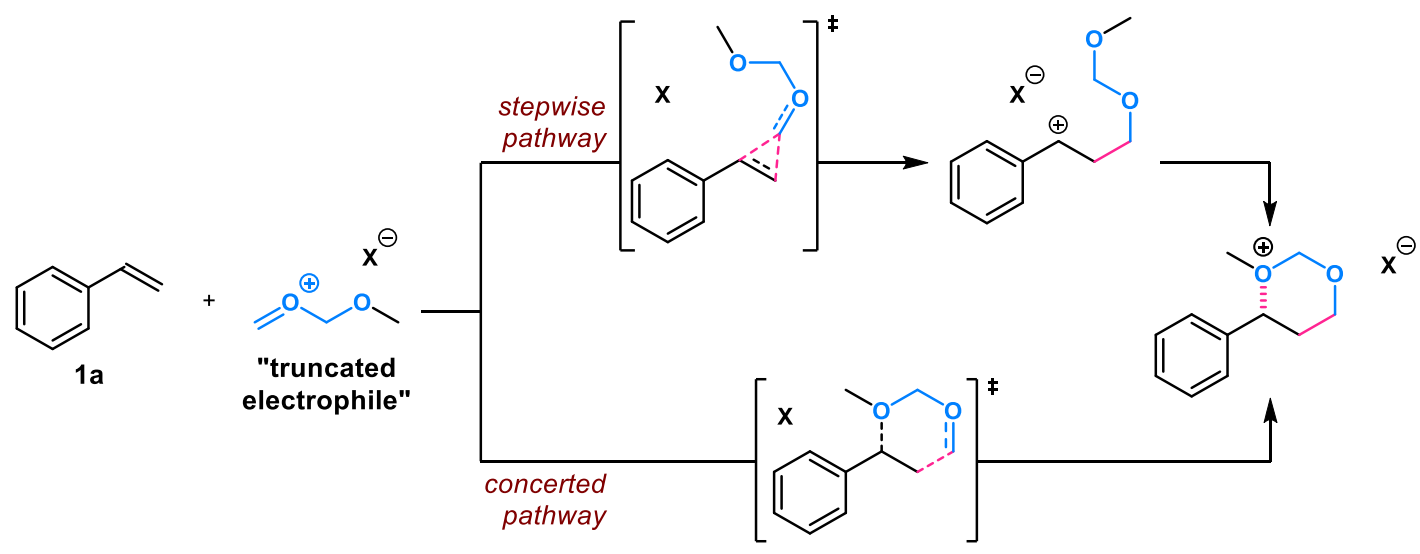

Scheme S-3. Effect of confinement on the operative reaction pathway. 
The results from the computations were the following (see Table S-4):

(a) With the $p$-toluenesulfonate anion:

A transition-state assembly TS-1 was found, resembling the structure of an "-onium cation", where

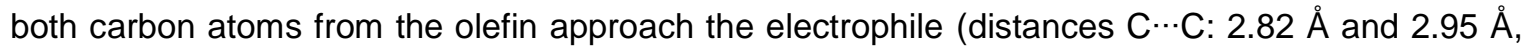
respectively). Notably, the bond distance between the benzylic carbon and the remote oxygen found to be $4.95 \AA$, ruling out any possibility of a concerted cyclization. This indicates that a stepwise mechanism is operative here via the stable benzyl cation as intermediate. Furthermore, changing the displacement variable along the imaginary vibration mode indicates that the observed TS indeed leads to a more stable non-cyclized intermediate $\mathbf{C} 1$. The intermediacy of such a carbocation matches both the observed differences in deuterium incorporation for the reaction with $(\mathrm{HCHO})_{n} /(\mathrm{DCDO})_{n}$ mixtures, and the cis-/trans-scrambling in the Prins reaction of $\beta$ deuterostyrenes.

\section{PTSA Catalyzed TS}

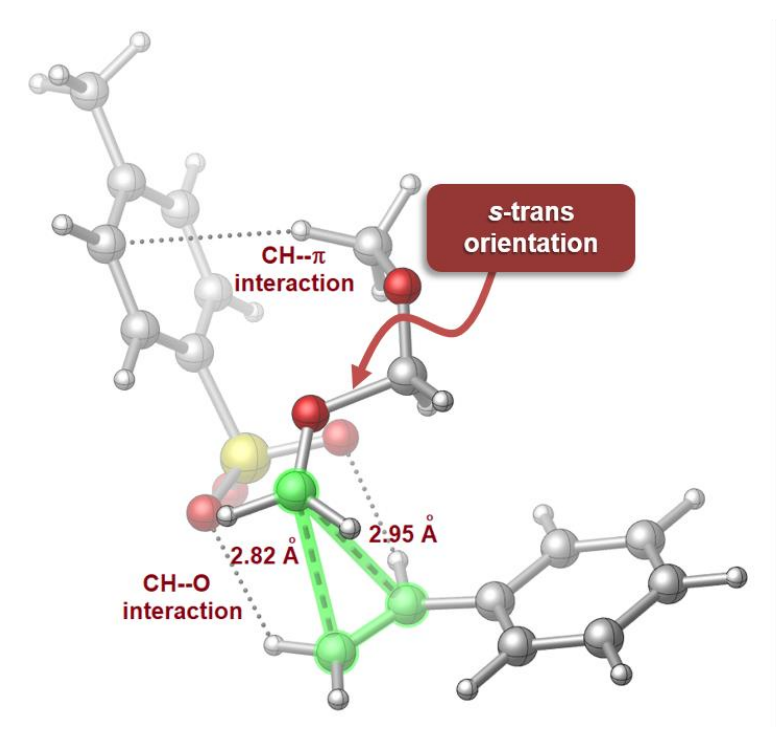

Product Complex

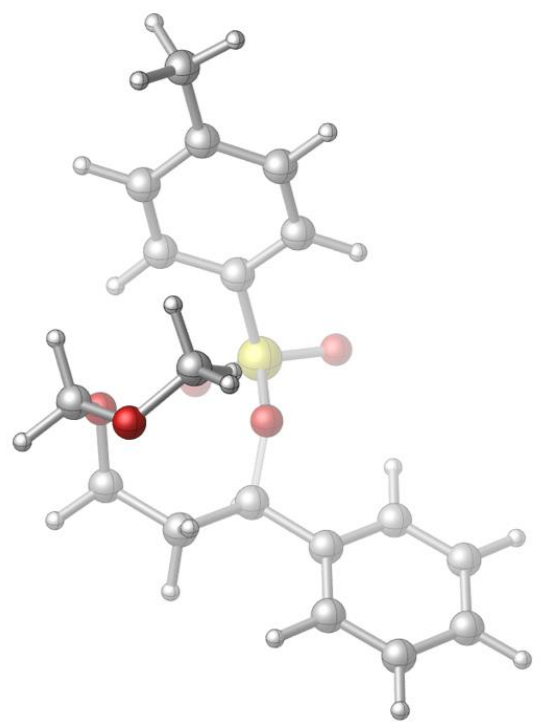

Figure S-3. TS-1 (left) and subsequent intermediate C1 (right), computed at the PBE-D3/def2-SVP level of theory.

(b) With the ilDP anion:

The nature of the TS is strikingly different in the presence of a confined, enantiopure $(S, S)$-ilDP anion (catalyst $\mathbf{4 b}$ ). In this case, we could only observe TS-2, where the distance between the atoms forming the $\mathrm{C}-\mathrm{C}$ bond is around $2 \AA$ (Figure S-4). A highly asynchronous, concerted chairlike TS structures was obtained where $\mathrm{C}-\mathrm{C}$ bond formation happens prior to the $\mathrm{O}-\mathrm{C}$ bond formation. Furthermore, the confined cavity induces the dimeric electrophile to adopt a conformation where the nucleophilic oxygen atom stays closer to the benzylic center ( $\mathrm{C} \cdots \mathrm{O}$ distance $=3.02 \AA$ ). By slight displacement from the TS structure toward product and subsequent optimization, a quick collapse of the TS to the formation of the $\mathrm{C}-\mathrm{O}$ bond is observed, that leads to direct cyclization (intermediate C2). This implies that the carbocationic intermediate is virtually non-existent within the catalyst cavity and the transformation follows rather a dynamically concerted pathway. This result is in agreement with the observed high stereospecificity for the iDP-catalyzed Prins reaction with $\beta$-deutero-styrenes. 


\section{TS}

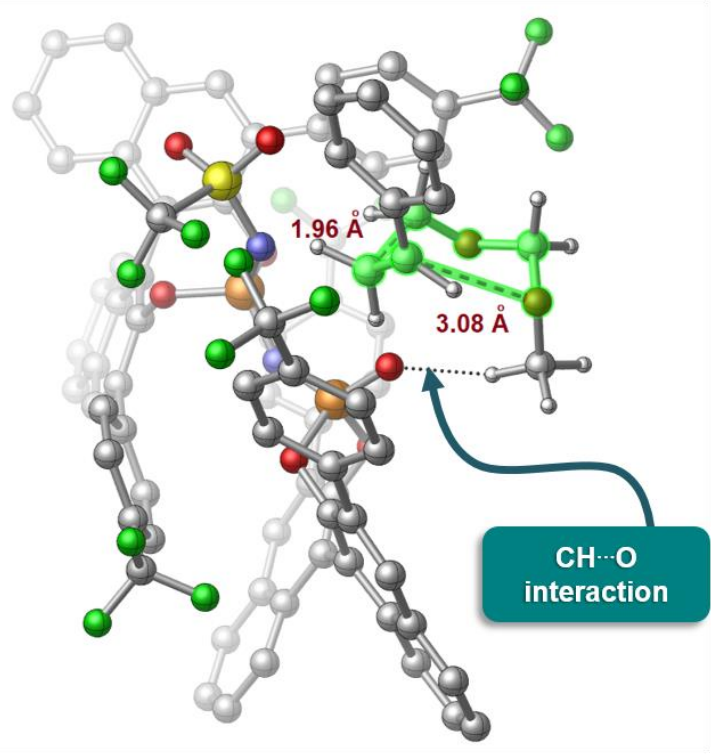

Pdt Complex

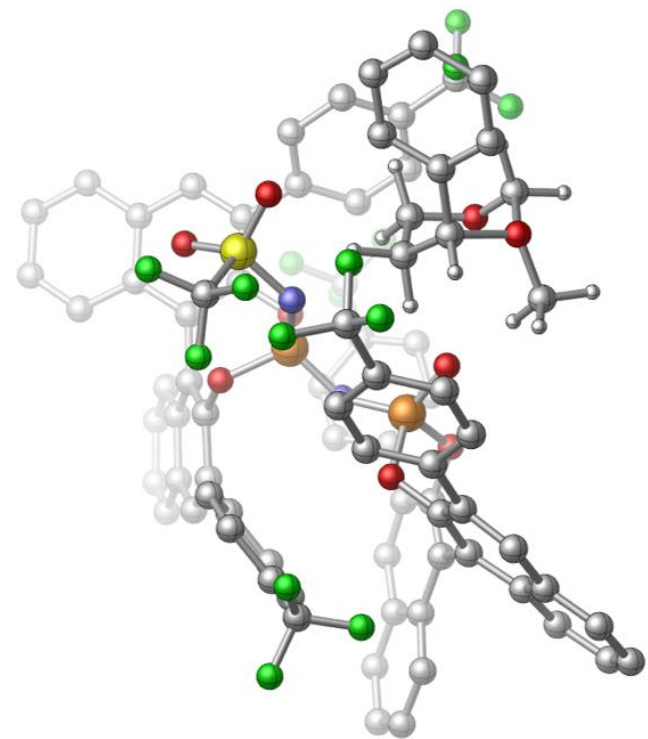

Figure S-4. TS-2 (left) and subsequent intermediate C2 (right), computed at the PBE-D3/def2-SVP level of theory.
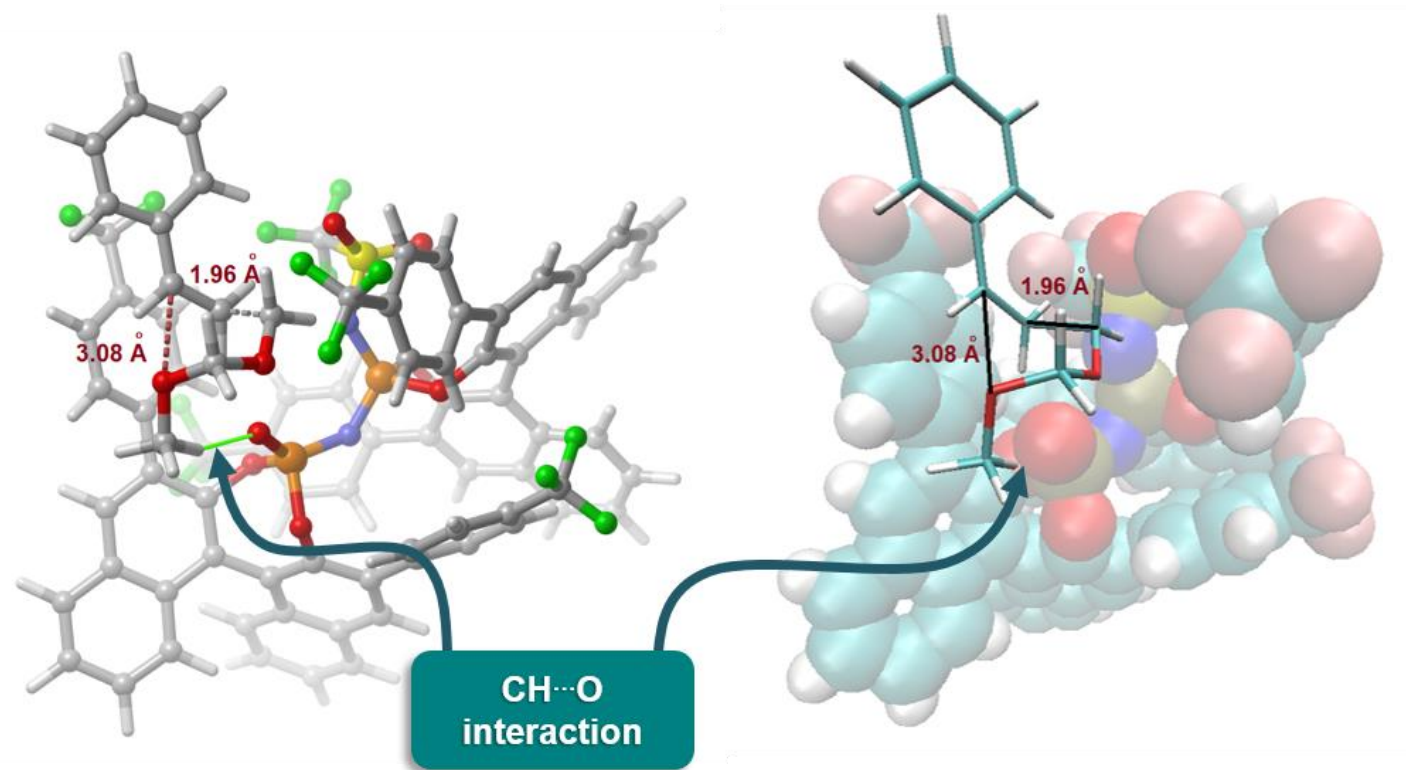

Figure S- 5. A different perspective of TS-2 (by rotating 180 degrees). The additional $\mathrm{CH} \cdots \mathrm{O}$ interaction within the confined cavity promotes an s-cis configuration of the electrophile. 
Table S-4. Comparison of p-TSOH and iIDP: TS energetics at the M06-2X/def2-TZVP+CPCM(cyclohexane)//PBE-D3/def2-SVP level of theory.

\begin{tabular}{|c|c|c|c|c|}
\hline TS No. & $\begin{array}{c}\text { PBE RRHO } \\
\text { corrections }\end{array}$ & $\begin{array}{c}\text { M06-2X/def2-TZVP } \\
\text { single point } \\
\text { (solvent: } \mathbf{C y H} \text { ) }\end{array}$ & $\begin{array}{c}\text { Imaginary } \\
\text { Freq. }\end{array}$ & $\begin{array}{c}\Delta \text { G(TS) } \\
\text { Final }\end{array}$ \\
\hline \multicolumn{5}{|c|}{ Catalyst: $p$-TsOH } \\
\hline TS-1 & 0.08029937 & -1473.3172402 & -118.36 & -1473.2369 \\
\hline \multicolumn{5}{|c|}{ Catalyst: ilDP 4b } \\
\hline TS-2 & 0.20778449 & -6444.62259 & -218.51 & -6444.4148 \\
\hline
\end{tabular}

\subsection{Understanding the Stereoselectivity}

Next, we turned our attention to understand the reason for the enantioselectivity in presence of catalyst $\mathbf{4 b}$, modeling the transition states toward the formation of each product enantiomer. Both TS structures adopt a chair-like conformation, where the initial $\mathrm{C}-\mathrm{C}$ bond formation precedes the subsequent $\mathrm{C}-\mathrm{O}$ collapse (Figure S-6). Based on the free energy difference of the optimized TS structures at $298 \mathrm{~K}$ (Table S-5), the predicted enantioselectivity (er = 99:1, at the M06-2X/def2-TZVP+ CPCM(cyclohexane)//PBE-D3/def2-SVP level of theory) is in good agreement with the experimental observation (er $=94.5: 5.5)$. Moreover, the $\mathrm{Ph}$ group in the stereodetermining TS is projected outside the cavity, explaining the high substrate group tolerance observed for this transformation. Employing the Distortion-Interaction (DI) analysis, we observed that the energy difference between two enantiodifferentiating TS structures (TS-2 and TS-3) originates from unfavorable distortion effects (see Table S-6). Presumably, such a distortion might cause due to the gain in additional $\mathrm{CH} \cdots \mathrm{F}$ interaction during the distorted structure. Notably, DI analysis revealed that much of the distortion was resulting from the twisted chair like substrate arrangement in the TS leading to the minor stereoisomer

\section{TS $_{\text {Major }}$}

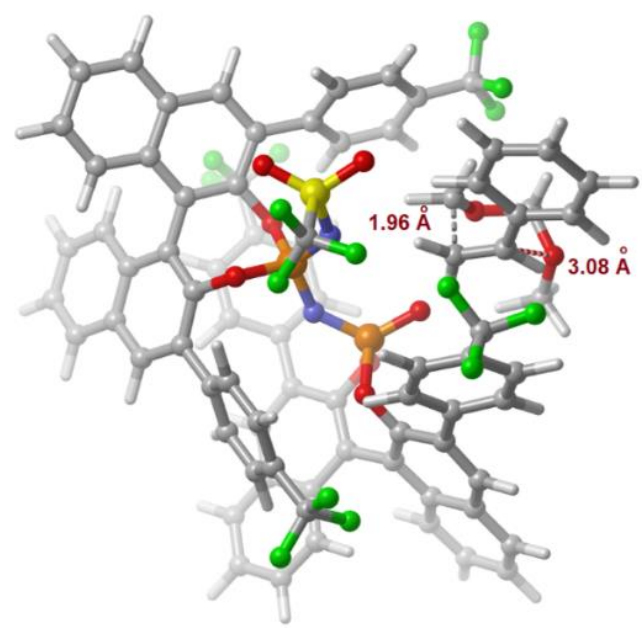

0.0

\section{TS ${ }_{\text {Minor }}$}

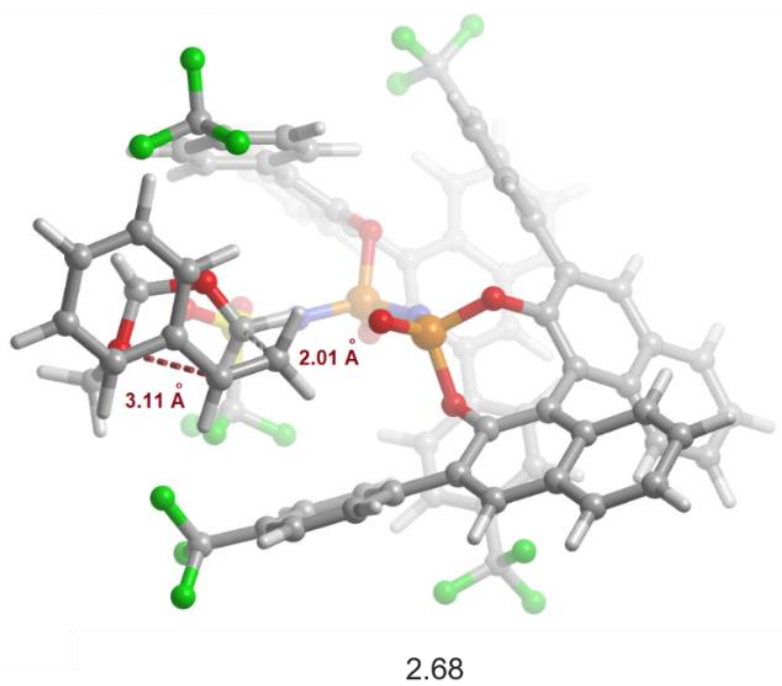

Figure S-6. Enantio-determining TS structures: highly asynchronous, concerted chair-like TS structures where C-C bond formation happens prior to the $\mathrm{O}-\mathrm{C}$ bond formation $\left(\Delta \Delta \mathrm{G}^{+}\right.$in $\mathrm{kcal} / \mathrm{mol}$, computed at the M06-2X/def2-TZVP+ CPCM(cyclohexane)//PBE-D3/def2-TZVP+CPCM(cyclohexane)//PBE-D3/def2-SVP level of theory). 
Notably, the catalyst counteranion leading to the minor isomer of the TS $((S)$-enantiomer of product) is more distorted to accommodate the substrate (Table S-6). This also highlights the importance of suitable cavity size in achieving the required stereoinduction.

Table S-5. TS energetics for the formation of each enantiomer of the Prins product, at the M06-2X/def2-TZVP+ CPCM(cyclohexane)//PBE-D3/def2-SVP level of theory.

\begin{tabular}{|c|c|c|c|c|c|}
\hline TS No. & $\begin{array}{c}\text { PBE RRHO } \\
\text { corrections }\end{array}$ & $\begin{array}{c}\text { M06-2X/def2-TZVP } \\
\text { single point } \\
\text { (solvent: } \text { CyH) }\end{array}$ & $\begin{array}{c}\text { Imaginary } \\
\text { Freq. }\end{array}$ & $\begin{array}{c}\Delta \text { G(TS) } \\
\text { Final }\end{array}$ & $\begin{array}{c}\text { Relative TS } \\
\text { Energy } \\
\text { (kcal/mol) }\end{array}$ \\
\hline \multicolumn{7}{|c|}{ Major enantiomer: $(R)$-3a } \\
\hline TS-2 & 0.20723631 & -6444.626477 & -303.94 & -6444.4191 & 0.0 \\
\hline \multicolumn{7}{|c|}{ Minor enantiomer: $(S)$-3a } \\
\hline TS-3 & 0.20778449 & -6444.62259 & -218.51 & -6444.4148 & 2.68 \\
\hline
\end{tabular}

Based on the optimized structures, the predicted enantioselectivity at the M06-2X/def2-TZVP+ CPCM(cyclohexane)//PBE-D3/def2-SVP level of theory at $298 \mathrm{~K}(\mathrm{er}=99: 1)$ is in good agreement with the experimentally observed enantioselectivity $(\mathrm{er}=94.5: 5.5)$.

Table S-6. Distortion-Interaction analysis to understand the selectivity for the intermolecular Prins reaction.

\begin{tabular}{|c|c|c|}
\hline TS No. & $\begin{array}{l}\text { M06-2X/def2-TZVP single point } \\
\text { (gas phase) }\end{array}$ & $\begin{array}{c}\text { Relative Energy } \\
\left(\Delta \Delta \mathrm{E}_{\text {gas }}{ }^{\ddagger}, \mathbf{k c a l} / \mathbf{m o l}\right)\end{array}$ \\
\hline TS-2 & -6444.604631 & \multirow[t]{2}{*}{2.3} \\
\hline TS-3 & -6444.600944 & \\
\hline \multicolumn{3}{|c|}{ Substrates Only $\left(\Delta \Delta \mathrm{E}_{\text {sub }^{\ddagger}}\right)$} \\
\hline Subst_TS-2 & -578.2826397 & \multirow[t]{2}{*}{1.8} \\
\hline Subst_TS-3 & -578.2797785 & \\
\hline \multicolumn{3}{|c|}{ Catalyst Only $\left(\Delta \Delta \mathrm{E}_{\text {sub }^{\ddagger}}\right)$} \\
\hline Cat_TS-2 & -5866.1760818 & \multirow[t]{2}{*}{0.31} \\
\hline Cat_TS-3 & -5866.1755331 & \\
\hline \multicolumn{2}{|c|}{$\begin{array}{l}\text { Total net Distortion in TS leading to minor enantiomer } \\
\left(\Delta \Delta \mathrm{E}_{\text {dist_total }}{ }^{\ddagger}\right)\end{array}$} & 2.11 \\
\hline Total Interaction & & 0.2 \\
\hline
\end{tabular}




\subsection{Optimized Cartesian Coordinates (PBE-D3/def2-SVP)}

TS-1

Imaginary frequency $=\mathbf{- 1 1 8 . 3 6}$

$-3.210091370 \quad 2.445729570$

$\begin{array}{lll}6 & -3.878021551 & 1.263455240\end{array}$

$\begin{array}{lll}6 & -1.363069515 & 0.363249689\end{array}$

$\begin{array}{lll}1 & -2.600244514 & 2.621436679\end{array}$

$\begin{array}{lll}1 & -1.716191108 & -0.606101640\end{array}$

$\begin{array}{lll}1 & -1.898678693 & 0.934241161\end{array}$

$\begin{array}{lll}1 & -3.794768531 & 0.549184652\end{array}$

$\begin{array}{lll}1 & -4.505487574 & 0.987669131\end{array}$

$6 \quad-3.131247154 \quad 3.489231942$

$\begin{array}{lll}6 & -2.395916903 & 4.665985368\end{array}$

$6 \quad-3.715564084 \quad 3.363627105$

$\begin{array}{lll}6 & -2.250269814 & 5.677933697\end{array}$

$\begin{array}{lll}1 & -1.938630721 & 4.772728775\end{array}$

$6 \quad-3.567799065 \quad 4.373823326$

$\begin{array}{lll}1 & -4.299705516 & 2.464190313\end{array}$

$\begin{array}{lll}6 & -2.834350208 & 5.535086418\end{array}$

$\begin{array}{lll}1 & -1.678042390 & 6.585705581\end{array}$

$\begin{array}{lll}1 & -4.031078087 & 4.258379705\end{array}$

$\begin{array}{lll}1 & -2.720488873 & 6.327834172\end{array}$

$\begin{array}{lll}1 & -0.191970100 & 2.768726722\end{array}$

$\begin{array}{lll}8 & 1.642528572 & 2.133900196\end{array}$

$8 \quad-0.234364225 \quad 0.751602790$

$\begin{array}{lll}1 & -0.045678090 & 2.358552746\end{array}$

$\begin{array}{lll}6 & 2.231642092 & 2.129213312\end{array}$

$\begin{array}{lll}1 & 1.519341999 & 2.530010610\end{array}$

$\begin{array}{lll}1 & 2.486754755 & 1.088820139\end{array}$

$\begin{array}{lll}1 & 3.152599698 & 2.739063418\end{array}$

$6 \quad 2.326861454-1.596067410$

$\begin{array}{lll}6 & 3.249564435 & -0.988350780\end{array}$

$\begin{array}{lll}6 & 2.810385934 & 0.114355028\end{array}$

$\begin{array}{lll}6 & 1.502364593 & 0.598911362\end{array}$

$\begin{array}{lll}6 & 0.606896061 & -0.013388563\end{array}$

$\begin{array}{lll}6 & 1.011705431 & -1.117797506\end{array}$

$\begin{array}{lll}1 & 2.644863080 & -2.464907348\end{array}$

$\begin{array}{lll}1 & 3.511871720 & 0.597507366\end{array}$

$1 \quad 1.159639655 \quad 1.452028553$

$\begin{array}{lll}1 & 0.286164081 & -1.592061289\end{array}$

$6 \quad 4.656585436 \quad-1.511910775$

$\begin{array}{lll}1 & 4.792268666 & -2.032136517\end{array}$

$1 \quad 4.908373766 \quad-2.233998579$

$1 \quad 5.402153722 \quad-0.691090569$

$\begin{array}{lll}16 & -1.034628695 & 0.717816856\end{array}$

$\begin{array}{lll}8 & -1.601204191 & 0.895102297\end{array}$

$\begin{array}{lll}8 & -0.730012780 & 2.031415180\end{array}$

$8 \quad-1.798983758 \quad-0.242888708$
$-4.772646504$

$-3.813448554$

$-3.887938443$

$-4.812650625$

$-2.908428959$

$-4.424772065$

$-5.594539834$

$-3.054697460$

$-4.753220614$

$-4.843029242$

$-4.557559103$

$-6.127972461$

$-5.515206722$

$-3.561098725$

$-7.085928996$

$-6.377186331$

$-6.784553947$

$-5.270521004$

$-8.077737089$

$-7.539276926$

$-3.987697593$

$-4.726747662$

$-4.400134999$

$-5.806026537$

$-3.405952143$

$-2.656681391$

$-3.120519308$

$-3.460383396$

$-1.736840092$

$-0.862354280$

$-0.094109592$

$-0.202145865$

$-1.096015320$

$-1.854646775$

$-2.335614475$

0.605350303

0.402244434

$-2.530451357$

$-0.719529056$

0.252810354

$-1.520369513$

$-0.748735630$

$-1.306169216$

0.052595099

$-2.014959988$

$-2.196740597$

\section{Pdt Complex of TS-1}

$\begin{array}{lrrr}6 & 1.904935274 & 1.195780359 & -5.945826078 \\ 6 & -2.183595069 & 1.154219433 & -5.129353783 \\ 6 & -1.253111596 & 1.703414690 & -6.207701061 \\ 6 & -0.274302118 & 0.685445063 & -6.807152178 \\ 1 & -2.740560205 & 0.290061751 & -5.556596905 \\ 1 & -0.805885348 & -0.259588630 & -7.039992011 \\ 1 & 0.128521349 & 1.099687811 & -7.762331168\end{array}$


The Catalytic Asymmetric Intermolecular Prins Reaction Computational Studies

\begin{tabular}{llll}
\hline 1 & -1.894370561 & 2.069380016 & -7.037648722 \\
1 & -0.685675830 & 2.575477599 & -5.825107441 \\
6 & -3.187900814 & 2.142490524 & -4.552826482 \\
6 & -3.988352427 & 1.732608812 & -3.465148112 \\
6 & -3.355546101 & 3.442529915 & -5.064972567 \\
6 & -4.938475020 & 2.600308470 & -2.912683112 \\
1 & -3.847799487 & 0.725010946 & -3.043617506 \\
6 & -4.309829269 & 4.311953195 & -4.510559824 \\
1 & -2.740180448 & 3.793340895 & -5.905195887 \\
6 & -5.104693671 & 3.894512102 & -3.434417855 \\
1 & -5.554759600 & 2.263240303 & -2.065141320 \\
1 & -4.426712400 & 5.325343587 & -4.924149778 \\
1 & -5.851341005 & 4.576454887 & -2.999678989 \\
1 & 2.163807085 & 1.518466170 & -6.982047268 \\
8 & 1.715357775 & 2.381852630 & -5.225252903 \\
8 & 0.810359440 & 0.314866041 & -5.963317723 \\
1 & 2.741249382 & 0.599838696 & -5.505807392 \\
6 & 1.610408945 & 2.187864580 & -3.819687721 \\
1 & 1.636032172 & 3.189625457 & -3.349583114 \\
1 & 0.664933970 & 1.676000750 & -3.543589184 \\
1 & 2.464791579 & 1.584220105 & -3.430614221 \\
6 & 1.526954847 & -0.915004104 & -0.977531512 \\
6 & 2.647355114 & -1.361074767 & -1.709227781 \\
6 & 2.467858367 & -1.715072631 & -3.065256748 \\
6 & 1.217140900 & -1.617461421 & -3.684401405 \\
6 & 0.128620291 & -1.165222708 & -2.924668974 \\
6 & 0.262368720 & -0.819090299 & -1.573187949 \\
1 & 1.647235112 & -0.642322389 & 0.082417858 \\
1 & 3.331120276 & -2.071330689 & -3.649431145 \\
1 & 1.075571504 & -1.858501363 & -4.745764663 \\
1 & -0.616911332 & -0.485508090 & -1.004037618 \\
6 & 4.006387335 & -1.468069231 & -1.067633498 \\
1 & 3.993147792 & -1.123151777 & -0.016282516 \\
1 & 4.371011624 & -2.516424849 & -1.078232410 \\
1 & 4.757126907 & -0.863524877 & -1.617624029 \\
16 & -1.467235256 & -0.994315406 & -3.698227065 \\
8 & -2.517393727 & -1.210431526 & -2.683126637 \\
8 & -1.383384438 & 0.644006025 & -4.014930723 \\
8 & -1.514684905 & -1.731984988 & -4.977291949
\end{tabular}

\begin{tabular}{llll}
\multicolumn{4}{l}{ Major Isomer } \\
\multicolumn{4}{l}{ TS-2 } \\
\multicolumn{4}{l}{ Imaginary frequency $=-303.94$} \\
15 & -0.350462660 & -0.707857142 & -0.915583153 \\
8 & 1.304920371 & -0.442088428 & -0.875304737 \\
8 & -0.401092100 & -2.158833370 & -0.094144834 \\
6 & 2.106661865 & -1.471763661 & -1.324712369 \\
6 & 2.683255150 & -1.377551937 & -2.634508182 \\
6 & 3.492737395 & -2.432592931 & -3.052841452 \\
6 & 3.703150434 & -3.592599035 & -2.260868885 \\
6 & 4.492995495 & -4.681615483 & -2.736651511 \\
6 & 4.647773043 & -5.832884973 & -1.981073810 \\
6 & 4.005526864 & -5.940784647 & -0.718856508 \\
6 & 3.246274670 & -4.892060319 & -0.219904400 \\
6 & 3.089977215 & -3.682149334 & -0.959096556 \\
6 & 2.323483310 & -2.564666583 & -0.476138952 \\
6 & 1.737057841 & -2.541124537 & 0.892434573 \\
6 & 2.553742004 & -2.670314868 & 2.070551533 \\
6 & 3.976887007 & -2.773352330 & 2.020174177
\end{tabular}


The Catalytic Asymmetric Intermolecular Prins Reaction Computational Studies

\begin{tabular}{|c|c|c|c|}
\hline 6 & 4.727537645 & -2.880975063 & 3.182560684 \\
\hline & 4.094613691 & -2.893525644 & 4.453983059 \\
\hline & .717266728 & -2.765889413 & 1.539005165 \\
\hline & .917061276 & -2.632419317 & 3.364651662 \\
\hline & 0.515134020 & -2.411367190 & 3.449674139 \\
\hline & .277308375 & -2.2169 & 320120299 \\
\hline & 0.364696808 & -2.310863199 & 1.042226951 \\
\hline & 2.461080860 & -0.211489797 & -3.526637446 \\
\hline 1 & 3.997439749 & -2.371286599 & -4.028826066 \\
\hline & 4.968653634 & -4.592350653 & -3.725870999 \\
\hline & 5.255136887 & -6.667998591 & 77780 \\
\hline 1 & 4.107351672 & -6.866217840 & -0.132055071 \\
\hline 1 & 2.748013989 & -4.989427657 & 0.754255394 \\
\hline 1 & 4.479643601 & -2.737912185 & 44422425 \\
\hline & 5.823900682 & -2.948319489 & 3.116980909 \\
\hline 1 & 4.700108194 & -2.987199328 & 3649 \\
\hline 1 & 2.215965761 & -2.748616390 & 5.519432872 \\
\hline 1 & 0.047714876 & -2.381164301 & 4.445784560 \\
\hline 6 & -1.712729870 & -1.8621 & 9521 \\
\hline 6 & 2.365701138 & 1.113045322 & 5867 \\
\hline 6 & 2.257352348 & 2.1997 & 5300 \\
\hline 6 & 2.239877083 & 1.9966 & 1404 \\
\hline 0 & 2.298570430 & 0.6873 & -5.804498068 \\
\hline 6 & 2.397426061 & -0.3990 & 6327 \\
\hline 6 & -2.11210 & -0.9680 & 9673 \\
\hline 6 & -3.448018934 & -0.5906 & 615944492 \\
\hline 6 & -4.41947 & -1.0892 & 9761 \\
\hline 6 & -4.043958059 & -1.9909 & 1.722683368 \\
\hline 6 & -2.7050883 & -2.3739 & 3829 \\
\hline 1 & 2.38474 & 1.2914 & 8604 \\
\hline 1 & 2.4201924 & -1.4196 & 29071 \\
\hline 1 & -1.354627071 & -0.5252 & 1718 \\
\hline 1 & -2.4228 & -3.0736 & 6311 \\
\hline 7 & -0.92432 & 240 & 6172 \\
\hline 15 & -1.7635 & 6608 & 4057 \\
\hline 8 & -1.17271 & $2.928 \mathrm{~s}$ & \\
\hline 8 & $-3.2117 \varepsilon$ & 1.4282 & 8291 \\
\hline 6 & -1.20088 & 7001 & 76657 \\
\hline 6 & 0.01254 & 2.3088 & 2821 \\
\hline 6 & -0.03661 & 713 & 1365 \\
\hline 6 & & & 8122 \\
\hline 6 & -1.282812001 & 1.933683782 & 1552 \\
\hline 6 & -2.475927448 & 2327 & 4605 \\
\hline 6 & -3.6833 & 2.299 & \\
\hline 6 & -3.6769159 & 2.5990 & 3292 \\
\hline 6 & -2.461677956 & 2.588839683 & 4.356596592 \\
\hline 6 & -2.414755513 & 2.8711 & 2.945409133 \\
\hline 6 & -3.629808846 & 3.256505261 & 413767 \\
\hline 6 & -4.413566319 & 72702 & 38850 \\
\hline 6 & -4.034118255 & 5.329096723 & 3.545990088 \\
\hline 6 & -4.821360715 & 6.430656858 & 3.849212493 \\
\hline 6 & -6.027657635 & 6.6745 & 118613 \\
\hline 6 & -6.409588161 & 5.821745237 & 2.117867615 \\
\hline 6 & -5.614086571 & 4.690238365 & 1.769704692 \\
\hline 6 & -5.962938678 & 3.854249097 & 0.675615898 \\
\hline 6 & -5.171350075 & 2.779339754 & 0.288741133 \\
\hline 6 & -4.006811551 & 2.498366764 & 1.066135978 \\
\hline 6 & & 2.080179010 & 2.148995572 \\
\hline 1 & 0.884617383 & 1.811340432 & 4.823606711 \\
\hline 1 & -0.343265948 & 1.663254527 & 6.938956796 \\
\hline
\end{tabular}


The Catalytic Asymmetric Intermolecular Prins Reaction Computational Studies

\begin{tabular}{|c|c|c|c|}
\hline 1 & -2.493084182 & 1.749822795 & 8.212147093 \\
\hline 1 & -4.634801257 & 2.303165051 & 7.013015177 \\
\hline 1 & -4.619793299 & 2.817490176 & 4.586872802 \\
\hline 1 & -3.097361598 & 5.159001367 & \\
\hline 1 & & & 4.641701088 \\
\hline 1 & -6.646822699 & 7.547910964 & 3.395294334 \\
\hline 1 & -7.329347246 & 6.012320543 & 3078 \\
\hline 1 & -6.876635613 & & 0.105717839 \\
\hline 6 & -5.486901177 & 2.006926538 & 302043 \\
\hline 6 & & & 3548 \\
\hline 6 & 2.860118344 & 2.706623630 & 34735 \\
\hline 0 & 3.629219166 & & 6017 \\
\hline 6 & & & 3788 \\
\hline 6 & 2.055792327 & 0.946388200 & 52813 \\
\hline 6 & -5.81 & 695 & 513 \\
\hline 6 & & & 4225 \\
\hline 6 & -6.1 & & 0104 \\
\hline 6 & & & 965 \\
\hline 6 & 94862 & & 1701 \\
\hline 1 & & & \\
\hline 1 & & & 844 \\
\hline 1 & 5088 & & 4398 \\
\hline 1 & & & \\
\hline 7 & & & 6602 \\
\hline 16 & & & 4120 \\
\hline 8 & -2.93412 & & 2214 \\
\hline 8 & & & 0865 \\
\hline 6 & & & -2 \\
\hline 9 & & & 863 \\
\hline 9 & -0.94 & & -2.5 \\
\hline 1 & & & \\
\hline 1 & & & \\
\hline 1 & & & \\
\hline 1 & & & 2078 \\
\hline 1 & & & 2373 \\
\hline 1 & & & 4581 \\
\hline 1 & & & 1749 \\
\hline 1 & -3.73 & & 893 \\
\hline 6 & -3.18 & -1.4 & 3840 \\
\hline 6 & & & \\
\hline 6 & & & 6939 \\
\hline 6 & 51932 & 3836 & 7050 \\
\hline 1 & & & \\
\hline 1 & & & 3642 \\
\hline 1 & -0.669683501 & & 3487 \\
\hline 1 & & & 75188 \\
\hline 6 & -1.195809302 & & 1396 \\
\hline 6 & & & 5438 \\
\hline 6 & & & 3405 \\
\hline 6 & & & -9.153957970 \\
\hline 1 & & & -8.503225942 \\
\hline 6 & -1.880218536 & & \\
\hline 1 & -1.888824992 & 3.594660901 & -5.368312530 \\
\hline 6 & & & -8.746984095 \\
\hline 1 & -0.953322610 & & -10.216659061 \\
\hline 1 & -2.249863766 & & \\
\hline 1 & & & -9.496425638 \\
\hline 8 & -0.876735121 & -0.949958273 & -2.300807036 \\
\hline 1 & & -2.329474001 & -5.144598075 \\
\hline & -1.917799466 & -1.874041361 & -5.763340684 \\
\hline
\end{tabular}


The Catalytic Asymmetric Intermolecular Prins Reaction Computational Studies

\begin{tabular}{lrrr}
\hline 8 & -3.149086014 & -0.708125553 & -4.152806823 \\
1 & -3.606500519 & -0.852018146 & -6.195724613 \\
6 & -1.314170861 & -2.838849167 & -4.886601581 \\
1 & -1.965907543 & -3.737572537 & -4.796254580 \\
1 & -0.356820021 & -3.132957770 & -5.355627032 \\
1 & -1.124496677 & -2.405473772 & -3.882128196 \\
9 & 0.129568549 & 4.447705949 & -1.264338275 \\
6 & -6.531419440 & -0.129542244 & -4.553123926 \\
6 & -5.846066200 & -0.631150102 & 2.886937636 \\
6 & 2.212298570 & 3.185928453 & -6.223559345 \\
6 & 4.876108269 & 1.244374190 & -0.090080188 \\
9 & -5.836393230 & 0.341233100 & -5.638877749 \\
9 & -7.841407022 & 0.019637122 & -4.855074847 \\
9 & -6.284277761 & -1.462361938 & -4.483810018 \\
9 & -6.646436605 & -1.089204477 & 1.891991107 \\
9 & -6.386853230 & -1.041294605 & 4.061055423 \\
9 & -5.935956026 & 0.728653375 & 2.875044934 \\
9 & 5.041844667 & 2.061932598 & -1.157633103 \\
9 & 5.993756415 & 1.343279013 & 0.672441512 \\
9 & 4.846765496 & -0.033219726 & -0.565469212 \\
9 & 1.922980613 & 2.834045801 & -7.503732680 \\
9 & 1.290292174 & 4.105943740 & -5.839617708 \\
9 & 3.408720423 & 3.823686356 & -6.249632691 \\
1 & -3.468708274 & 1.138792599 & -5.059631103
\end{tabular}

\section{Pdt Complex of TS-2}

$\begin{array}{llll}15 & -0.225277632 & -0.680068078 & -0.972679886 \\ 8 & 1.424072934 & -0.421800409 & -0.813249345 \\ 8 & -0.322946432 & -2.146897613 & -0.182876001 \\ 6 & 2.269932429 & -1.432871618 & -1.213977968 \\ 6 & 2.953455641 & -1.302545907 & -2.469223410 \\ 6 & 3.824488687 & -2.328276617 & -2.831523459 \\ 6 & 3.988272189 & -3.500061593 & -2.045662681 \\ 6 & 4.841246129 & -4.562779105 & -2.469302561 \\ 6 & 4.946965509 & -5.729804176 & -1.729638571 \\ 6 & 4.189999008 & -5.881116397 & -0.537353238 \\ 6 & 3.367472927 & -4.858163472 & -0.087678376 \\ 6 & 3.258960529 & -3.632184791 & -0.808629614 \\ 6 & 2.433591980 & -2.538470610 & -0.369455765 \\ 6 & 1.743065288 & -2.542798130 & 0.950191087 \\ 6 & 2.470936236 & -2.688785815 & 2.184773797 \\ 6 & 3.893363222 & -2.801079455 & 2.240878365 \\ 6 & 4.555134185 & -2.926388178 & 3.454479786 \\ 6 & 3.830130506 & -2.947017761 & 4.675363324 \\ 6 & 2.451315172 & -2.809463705 & 4.658625667 \\ 6 & 1.741725104 & -2.658938924 & 3.429391795 \\ 6 & 0.339157605 & -2.427952689 & 3.414482133 \\ 6 & -0.368167560 & -2.223113074 & 2.232074687 \\ 6 & 0.362873934 & -2.310624377 & 1.002447038 \\ 6 & 2.738983662 & -0.137513499 & -3.363726364 \\ 1 & 4.412075351 & -2.238109041 & -3.757730276 \\ 1 & 5.406077294 & -4.440620459 & -3.406784867 \\ 1 & 5.604156215 & -6.544156514 & -2.069781134 \\ 1 & 4.253503517 & -6.819487958 & 0.034072674 \\ 1 & 2.781924773 & -4.987880932 & 0.832746254 \\ 1 & 4.469169718 & -2.762605119 & 1.306550995 \\ 1 & 5.652802373 & -3.002468580 & 3.469049360 \\ 1 & 4.365456567 & -3.054508847 & 5.630622749 \\ 1 & 1.877917144 & -2.798302155 & 5.598784033 \\ 1 & -0.199042455 & -2.399403558 & 4.374211789\end{array}$


The Catalytic Asymmetric Intermolecular Prins Reaction Computational Studies

\begin{tabular}{|c|c|c|c|}
\hline 6 & -1.809451905 & -1.869848451 & 2.263874409 \\
\hline & 2.495774356 & 1.164719381 & -2.866473656 \\
\hline 6 & & & \\
\hline & 2.347746900 & & \\
\hline 0 & & & 6343 \\
\hline 6 & 2.795912994 & -0.304667699 & -4.768531655 \\
\hline 6 & -2.273219179 & & 3.250303942 \\
\hline 6 & -3.619374126 & & 3.321434461 \\
\hline & -4.536260319 & -1.129994495 & 2.393528119 \\
\hline 0 & $-4.094719 s$ & -2.02 & 1.40 \\
\hline 6 & -2.744764724 & & \\
\hline & 2.454983732 & 1.335861407 & 5781023 \\
\hline 1 & & & 20729 \\
\hline 1 & -1.5 & & 13500 \\
\hline 1 & 3700 & & \\
\hline 7 & -0.8 & & 6848 \\
\hline 15 & -1.644365151 & 167289 & -0.128092666 \\
\hline 8 & -1.0 & & 969 \\
\hline 8 & -3.1 & & \\
\hline$b$ & & & \\
\hline 6 & & & \\
\hline ? & 0.0 & & \\
\hline 6 & & & \\
\hline 6 & & & \\
\hline 6 & & & 019 \\
\hline 6 & & & \\
\hline 6 & & & \\
\hline 6 & & & \\
\hline 6 & -2.3 & & \\
\hline 6 & & & \\
\hline 6 & & & \\
\hline 6 & -3.8 & & \\
\hline 6 & -4.6 & & \\
\hline 6 & & & \\
\hline 6 & & & \\
\hline 6 & & & \\
\hline 6 & -5.8 & & 5727 \\
\hline & -5.0 & & \\
\hline 6 & & & 442 \\
\hline 6 & & & \\
\hline 1 & & & \\
\hline 1 & -0.3 & & \\
\hline 1 & & & \\
\hline 1 & -4.60 & & \\
\hline 1 & -4.548719315 & 2.72040 & 6369 \\
\hline 1 & & & \\
\hline 1 & -4.3327 & & 5113 \\
\hline 1 & -6.459135193 & & \\
\hline 1 & & & \\
\hline 1 & -6.739416767 & & \\
\hline 6 & -5.398377352 & & \\
\hline 6 & & & \\
\hline 6 & 3.001677315 & 2.687320090 & 0.480310845 \\
\hline 6 & & & \\
\hline 6 & & & 993107 \\
\hline 6 & 2.157307595 & 0.869219823 & 2.434717111 \\
\hline 6 & & & \\
\hline 6 & -6.148342768 & 2.248362093 & -3.329966096 \\
\hline 6 & -6.131839127 & 0.843520661 & -3.400684290 \\
\hline
\end{tabular}

$\begin{array}{llll}6 & -5.737916635 & 0.092904433 & -2.280258004\end{array}$ 
The Catalytic Asymmetric Intermolecular Prins Reaction Computational Studies

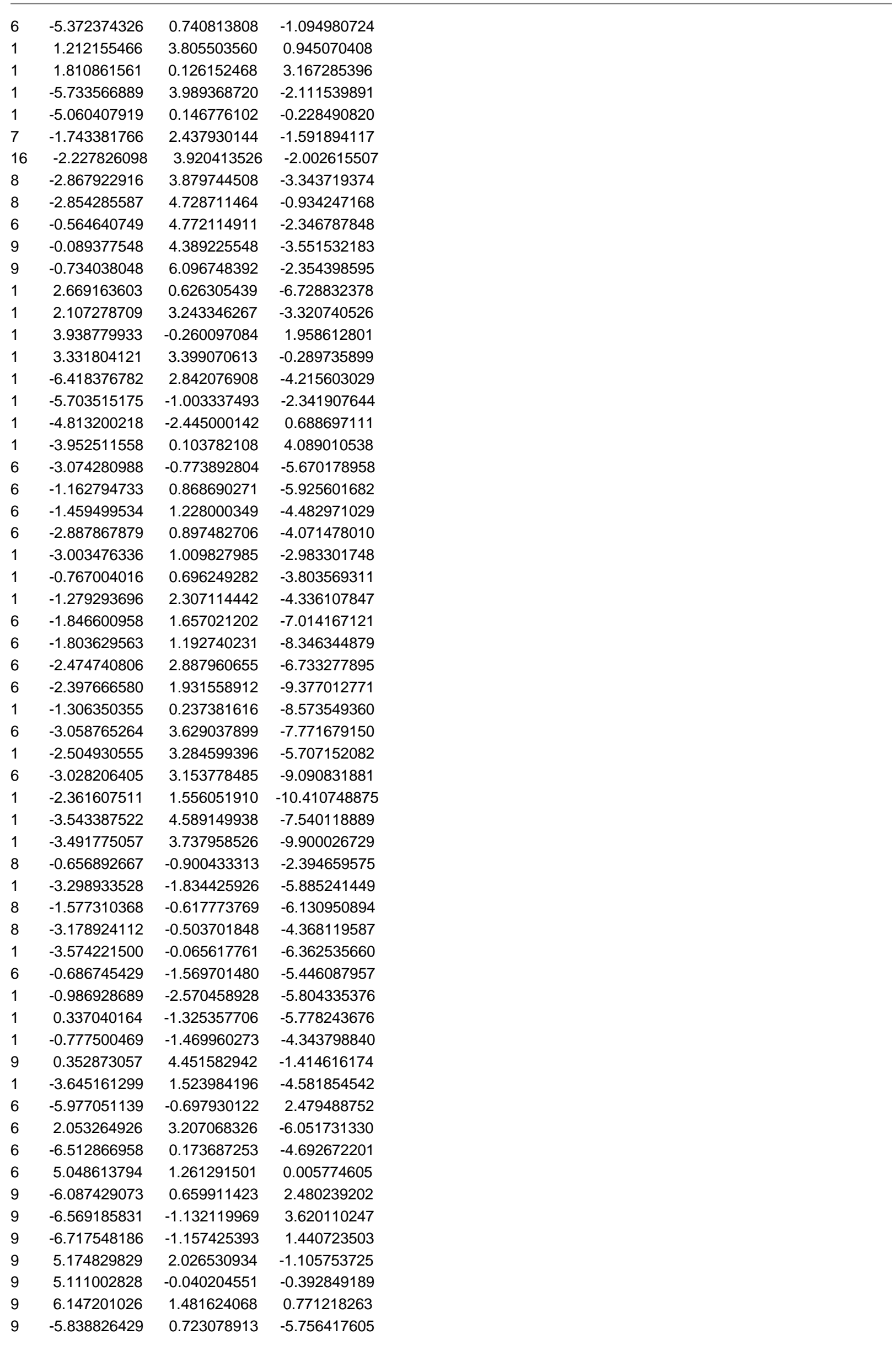


The Catalytic Asymmetric Intermolecular Prins Reaction Computational Studies

\begin{tabular}{rrrr}
\hline 9 & -7.829081094 & 0.309792451 & -4.976289757 \\
9 & -6.232857796 & -1.152380092 & -4.703080983 \\
9 & 2.458118351 & 4.390738299 & -5.547199532 \\
9 & 2.639054176 & 3.047766239 & -7.262820388 \\
9 & 0.708117959 & 3.317610120 & -6.284662801 \\
1 & -0.071652148 & 0.818995950 & -6.109600953
\end{tabular}

TS-3

Imaginary frequency $=\mathbf{- 2 1 8 . 5 1}$

$15 \quad 0.253838804-0.454155665$

$8-1.896310758-0.267044521$

$\begin{array}{lll}8 & 0.068537367 & -2.002785687\end{array}$

$\begin{array}{lll}6 & 2.767344764 & -1.256077453\end{array}$

$6 \quad 3.606149452-1.014353934$

$\begin{array}{lll}6 & 4.501633952 & -2.020067913\end{array}$

$\begin{array}{lll}6 & 4.553186828 & -3.270014912\end{array}$

$\begin{array}{lll}6 & 5.435412045 & -4.306525181\end{array}$

$\begin{array}{lll}6 & 5.439076607 & -5.542658614\end{array}$

$\begin{array}{lllll}6 & 4.547930052 & -5.791008911 & -0.6\end{array}$

$\begin{array}{llll}6 & 3.691982210 & -4.797434008 & -0.1\end{array}$

$6 \quad 3.682320211 \quad-3.505342853$

$\begin{array}{lll}6 & 2.825563682 & -2.440464094\end{array}$

$\begin{array}{llll}6 & 2.011970474 & -2.553519085\end{array}$

$\begin{array}{lll}6 & 2.622453815 & -2.825652239\end{array}$

$\begin{array}{lll}6 & 4.033389354 & -2.958515047\end{array}$

$\begin{array}{llll}6 & 4.579979119 & -3.204632007\end{array}$

$\begin{array}{lll}6 & 3.745467437 & -3.332388086\end{array}$

$\begin{array}{lll}6 & 2.374421048 & -3.178156848\end{array}$

$\begin{array}{lll}6 & 1.781888264 & -2.903946164\end{array}$

$\begin{array}{lll}6 & 0.387738123 & -2.655160262\end{array}$

$\begin{array}{lll}6 & -0.205440681 & -2.325606089\end{array}$

$\begin{array}{lll}6 & 0.636060689 & -2.303063477\end{array}$

$\begin{array}{lll}6 & 3.530566758 & 0.242696054\end{array}$

$1 \quad 5.199564755 \quad-1.850251361$

$1 \quad 6.106090956 \quad-4.107045097$

$1 \quad 6.119575492 \quad-6.336115277$

$1 \quad 4.532613633 \quad-6.782149644$

$\begin{array}{lll}1 & 3.002351307 & -5.002168664\end{array}$

$1 \quad 4.693124155 \quad-2.833391945$

$\begin{array}{lll}1 & 4.693124155 & -2.833391945 \\ 1 & 5.671571468 & -3.292035924\end{array}$

$1 \quad 4.190175957 \quad-3.535609633$

$1 \quad 1.716932140 \quad-3.248276948$

$\begin{array}{lll}1 & -0.237956685 & -2.714170887\end{array}$

$6 \quad-1.642765413 \quad-1.961494770$

$\begin{array}{lll}6 & 3.233208493 & 1.494479564\end{array}$

$\begin{array}{lll}6 & 3.158715755 & 2.657224358\end{array}$

$\begin{array}{lll}6 & 3.390474092 & 2.603952086\end{array}$

$6 \quad 3.716029250 \quad 1.373812106$

$\begin{array}{lll}6 & 3.772758586 & 0.212530891\end{array}$

$6 \quad-2.194073854 \quad-1.152903791$

$6 \quad-3.541708956 \quad-0.788703615$

$\begin{array}{lll}6 & -3.541708956 & -0.788703615 \\ 6 & -4.371011238 & -1.216953778\end{array}$

$\begin{array}{lll}6 & -3.842829803 & -2.030204938\end{array}$

$\begin{array}{lll}6 & -2.491570938 & -2.395670478\end{array}$

$\begin{array}{lll}1 & 3.048923292 & 1.560198015\end{array}$

$\begin{array}{lll}1 & 3.982828364 & -0.748453765\end{array}$

$1 \quad-1.543955459-0.765549026$

$\begin{array}{lll}1 & -2.086606997 & -3.023924332\end{array}$

$\begin{array}{lll}7 & -0.385154424 & 0.541678701\end{array}$
$-0.961983513$

$-0.685069088$

$-0.371781025$

$-1.084121068$

$-2.223563299$

$-2.583419239$

$-1.911892945$

$-2.340449890$

$-1.714342600$

$-0.636499365$

$-0.183497660$

$-0.787525345$

$-0.338384582$

0.903139187

2.179561161

2.354197242

3.606029316

4.748000168

4.618360531

3.349150487

3.227322600

2.010916081

0.851075921

$-3.009209242$

$-3.416994114$

$-3.190818055$

$-2.057978365$

$-0.158470438$

0.646819844

1.485660386

3.713998068

5.733702546

5.499055344

4.131007489

1.947388296

$-2.420132431$

$-3.194518925$

$-4.578848701$

$-5.180150589$

$-4.405113105$

2.967165045

2.949982733

1.899984586

0.883531456

0.904954055

$-1.342557284$

$-4.896369148$

3.765024273

0.101286700

0.119557104 
The Catalytic Asymmetric Intermolecular Prins Reaction Computational Studies

\begin{tabular}{|c|c|c|c|}
\hline 5 & -1.236279475 & 1.861645793 & \\
\hline & -0.800302892 & 2.933625221 & \\
\hline & -2.753275401 & .449023179 & 0.549791281 \\
\hline & -1.035589665 & 2.571484590 & 2.520278792 \\
\hline & 0.072460705 & 2.091738764 & 3.288967473 \\
\hline 0 & -0.176018758 & 19261 & 342342 \\
\hline & -1.480512533 & 1.779573625 & 5.174743119 \\
\hline & -1.723079619 & 1.347411165 & 6.513252078 \\
\hline 6 & -3.007522283 & 1.319201307 & \\
\hline & -4.105668075 & 1.714559656 & 6.223486164 \\
\hline 6 & -3.9025821 & 2.158 & $4.9244 \varepsilon$ \\
\hline 6 & -2.589829977 & 2.227724319 & 4.367798910 \\
\hline & -2.338921633 & 2.662693335 & 3.018057513 \\
\hline 6 & -3.436340 & 3.118483342 & \\
\hline 6 & -4.288092195 & 4.229225726 & 2.4499 \\
\hline b & -4.089031465 & 5.041 & 3.60 \\
\hline 6 & -4.941642826 & 6.098 & \\
\hline 6 & -6.035930802 & 6.395 & 3.0355 \\
\hline 6 & -6.239956 & & \\
\hline 6 & -5.3712938 & 4.56 & \\
\hline 6 & -5.535871720 & 3.83 & 0.3 \\
\hline 6 & -4.668852 & 2.806 & \\
\hline b & -3.6279355 & 2.467 & 0.8991 \\
\hline & $1.416101 \varepsilon$ & $1.92 C$ & \\
\hline 1 & 0.6582068 & 1.38 & \\
\hline 1 & -0.8654362 & 1.02 & \\
\hline 1 & -3.180766 & 368 & \\
\hline 1 & -5.1284683 & 1.661 & 6.6257 \\
\hline 1 & -4.761169 & 2.438 & \\
\hline 1 & -3.239553 & 4.82 & \\
\hline 1 & -4.763784 & 94884 & 4.78 \\
\hline 1 & -6.709214 & 884 & \\
\hline 1 & -7.070270 & & \\
\hline 1 & -6.371843 & 4.09 & -0.3 \\
\hline 6 & -4.791582 & & \\
\hline 6 & 1.985012 & & \\
\hline 6 & 3.239174 & 838 & \\
\hline 6 & 3.950196 & 153 & \\
\hline 6 & 3.406314 & 0.533 & \\
\hline 6 & $2.151352 \varepsilon$ & 63 & \\
\hline 6 & & & \\
\hline 6 & -5.282944625 & 2.3297 & \\
\hline 6 & -50475971 & 8890 & 3756 \\
\hline 6 & -4.684712 & 263 & \\
\hline 6 & -4.565058 & 884 & -1.5 \\
\hline 1 & 1.435430895 & 96853 & 9496 \\
\hline 1 & 1.710908313 & -0.0397 & \\
\hline 1 & -5.258941803 & 29994 & -2.368797546 \\
\hline 1 & -4.283290405 & 3563 & -0.6 \\
\hline 7 & -1.247739962 & 2.649130525 & -1.391144480 \\
\hline 16 & -1.631178624 & 4.193167440 & -1.637011259 \\
\hline 8 & -2.214324507 & 4.345995238 & -2.996303269 \\
\hline 8 & -2.252121399 & 4.920752660 & -0.511368247 \\
\hline 6 & 0.092033677 & 4.968326808 & -1.821085542 \\
\hline 9 & 0.922460689 & 4.553152639 & \\
\hline 9 & 0.637578041 & 4.617991227 & -3.011323827 \\
\hline 1 & 3.910891269 & 1.323312105 & -6.261845819 \\
\hline 1 & & 3.614395417 & -2.717185401 \\
\hline 1 & 3.950772962 & -0.398157973 & 2.581045288 \\
\hline 1 & -5.549948150 & 2.947453209 & -4.604433596 \\
\hline
\end{tabular}


The Catalytic Asymmetric Intermolecular Prins Reaction Computational Studies

\begin{tabular}{llll}
\hline 1 & -4.487739713 & -0.900080613 & -2.910636782 \\
1 & -4.494297476 & -2.374672055 & 0.067768297 \\
1 & -3.942732207 & -0.143395936 & 3.743804201 \\
6 & -2.387211068 & 3.054157502 & -5.739330826 \\
6 & 0.313784752 & 0.939949736 & -6.426654098 \\
6 & 0.202670891 & 0.352573382 & -5.177913561 \\
6 & -0.917788719 & 1.780108952 & -4.296148711 \\
1 & -0.892126373 & 1.127729435 & -3.398136586 \\
1 & -0.233633617 & 2.643212489 & -4.346657202 \\
1 & -0.502836694 & -0.469150505 & -4.984311509 \\
1 & 1.049705367 & 0.387377019 & -4.477809176 \\
8 & -0.125709585 & -0.454859001 & -2.415401245 \\
1 & -2.985544460 & 3.733656814 & -5.098726943 \\
8 & -1.247501811 & 3.632158687 & -6.233883394 \\
8 & -2.095118331 & 1.894372792 & -4.856399060 \\
1 & -2.994323888 & 2.595578744 & -6.543026582 \\
6 & -0.982986998 & 4.994408587 & -5.863614176 \\
1 & -1.290523707 & 5.192613720 & -4.818070621 \\
1 & 0.106911520 & 5.143579431 & -5.967646544 \\
1 & -1.513752002 & 5.686254325 & -6.553196154 \\
9 & -0.003386088 & 6.300492011 & -1.777592517 \\
6 & -5.225372892 & 0.307575789 & -5.244550559 \\
6 & -5.814207842 & -0.783004790 & 1.888450875 \\
6 & 3.247445996 & 3.829243847 & -5.439620531 \\
9 & -6.467046626 & -0.211357347 & -5.406224720 \\
9 & -4.350562043 & -0.718202087 & -5.444121130 \\
9 & -5.040033965 & 1.189889903 & -6.269232000 \\
9 & 2.105191025 & 3.757229846 & -6.211390164 \\
9 & 3.152360705 & 4.968131912 & -4.725877371 \\
9 & 5.272703066 & 3.965310412 & -6.311783671 \\
9 & 5.353300387 & -0.026796273 & 0.342036445 \\
9 & 6.302912498 & 1.310551115 & 1.774764466
\end{tabular}




\section{Copies of NMR spectra}
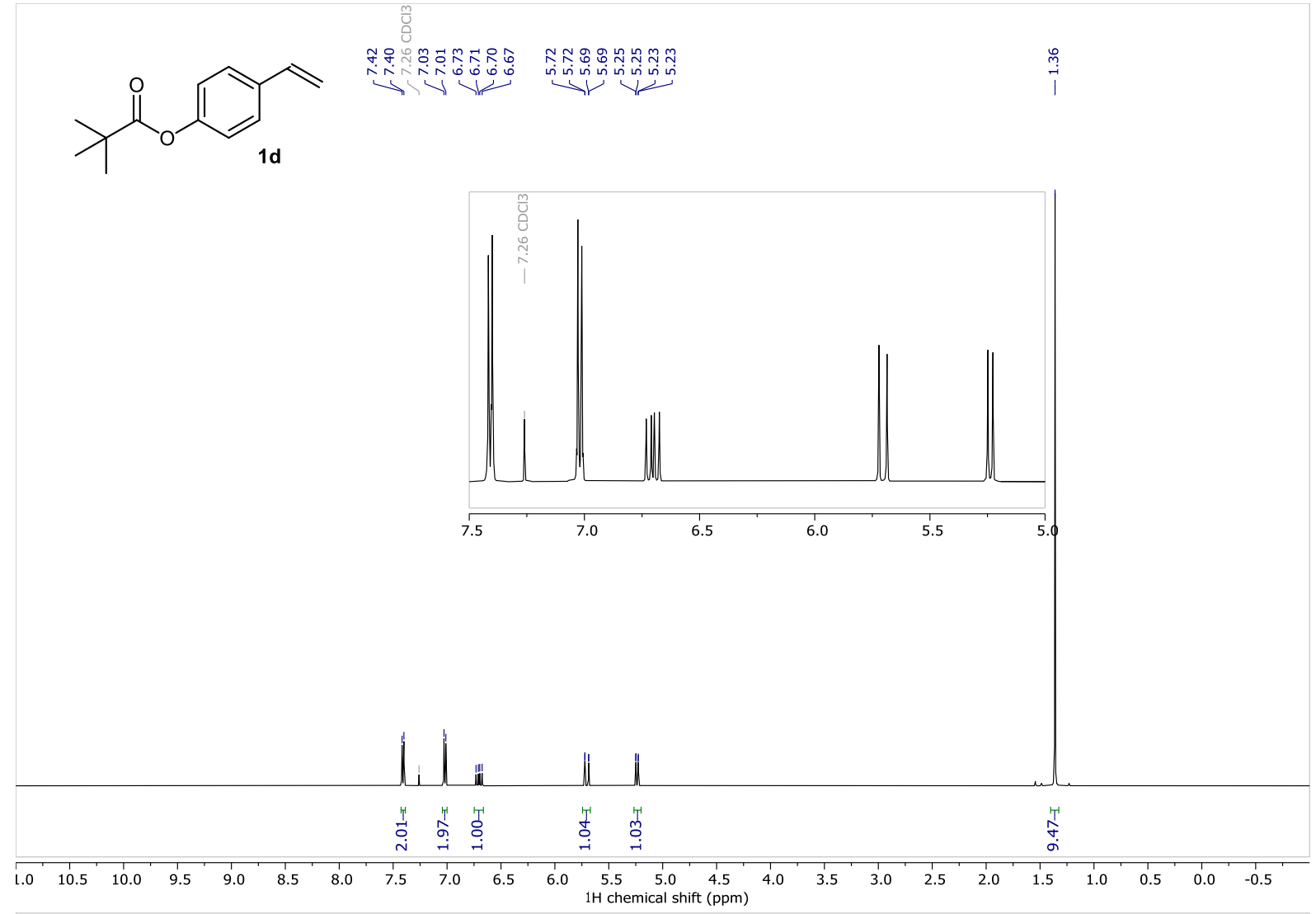

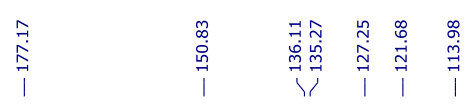<smiles>C=Cc1ccc(OC(=O)C(C)(C)C)cc1</smiles>
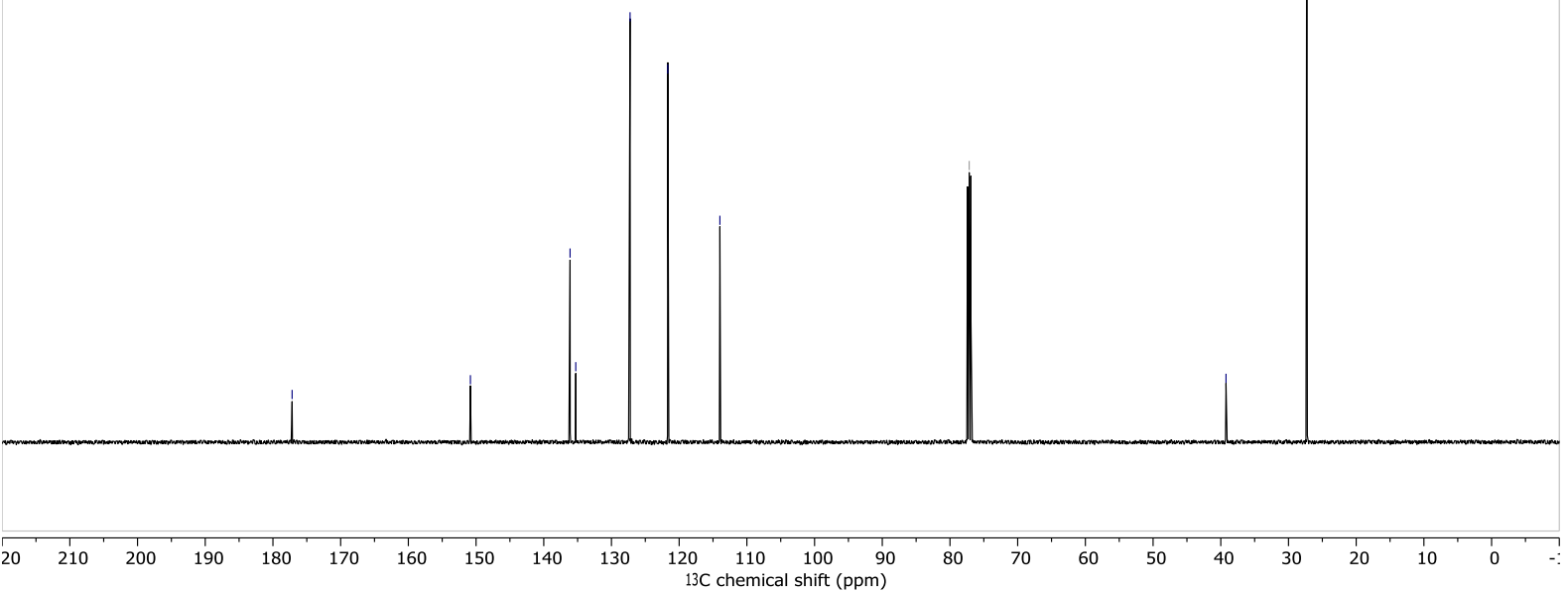

NMR spectra for compound $1 \mathrm{~d}:{ }^{1} \mathrm{H}(501 \mathrm{MHz})$ and ${ }^{13} \mathrm{C}(126 \mathrm{MHz})$, in $\mathrm{CDCl}_{3}$. 


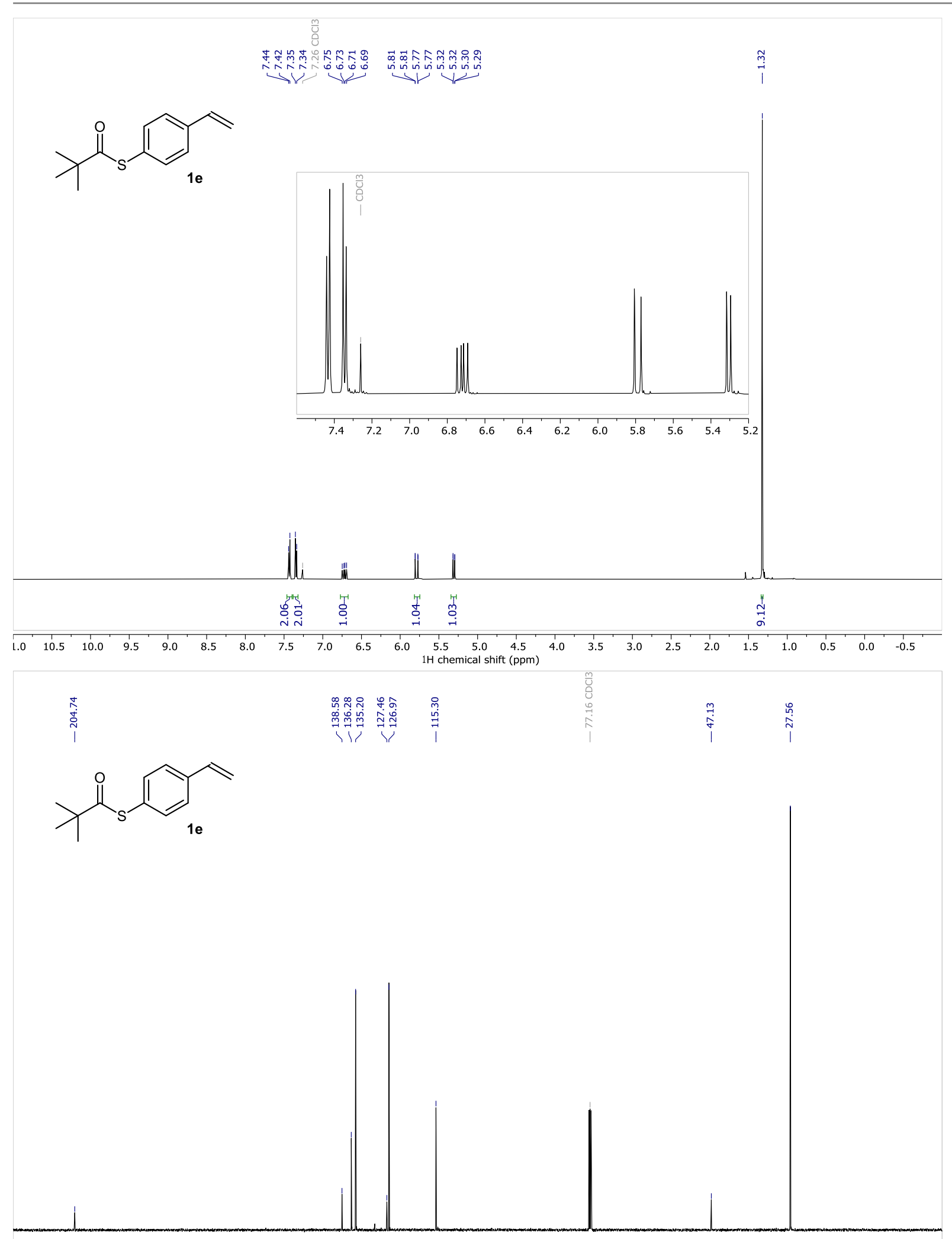

$\begin{array}{llllllllllll}210 & 200 & 190 & 180 & 170 & 160 & 150 & 140 & 130 & 120 & 110 & 100 \\ 13 \mathrm{C} \text { chemical shift (ppm) }\end{array}$

NMR spectra for compound 1e: ${ }^{1} \mathrm{H}(501 \mathrm{MHz})$ and ${ }^{13} \mathrm{C}(126 \mathrm{MHz})$, in $\mathrm{CDCl}_{3}$. 


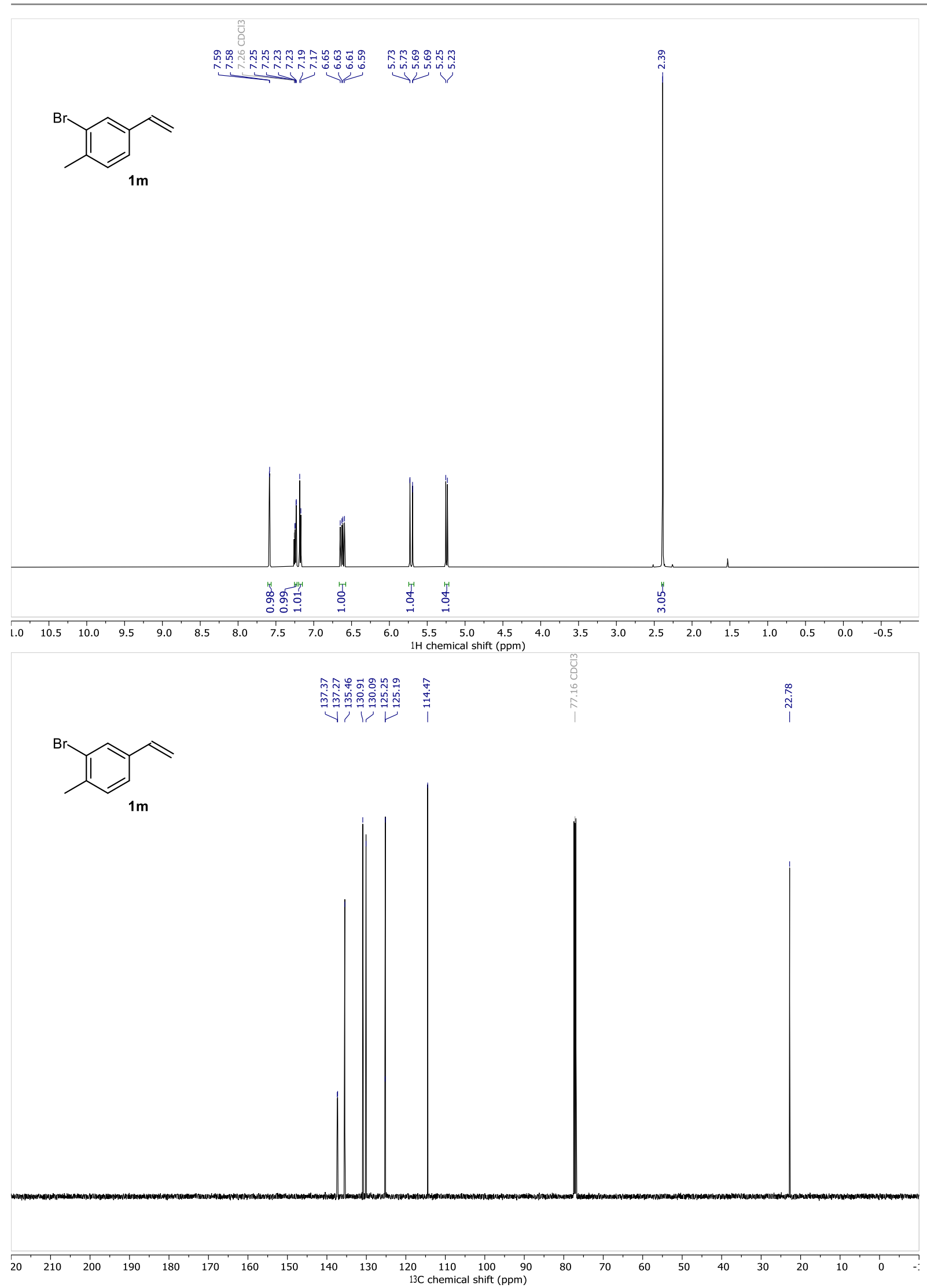

NMR spectra for compound $1 \mathrm{~m}:{ }^{1} \mathrm{H}(501 \mathrm{MHz})$ and ${ }^{13} \mathrm{C}(126 \mathrm{MHz})$, in $\mathrm{CDCl}_{3}$. 


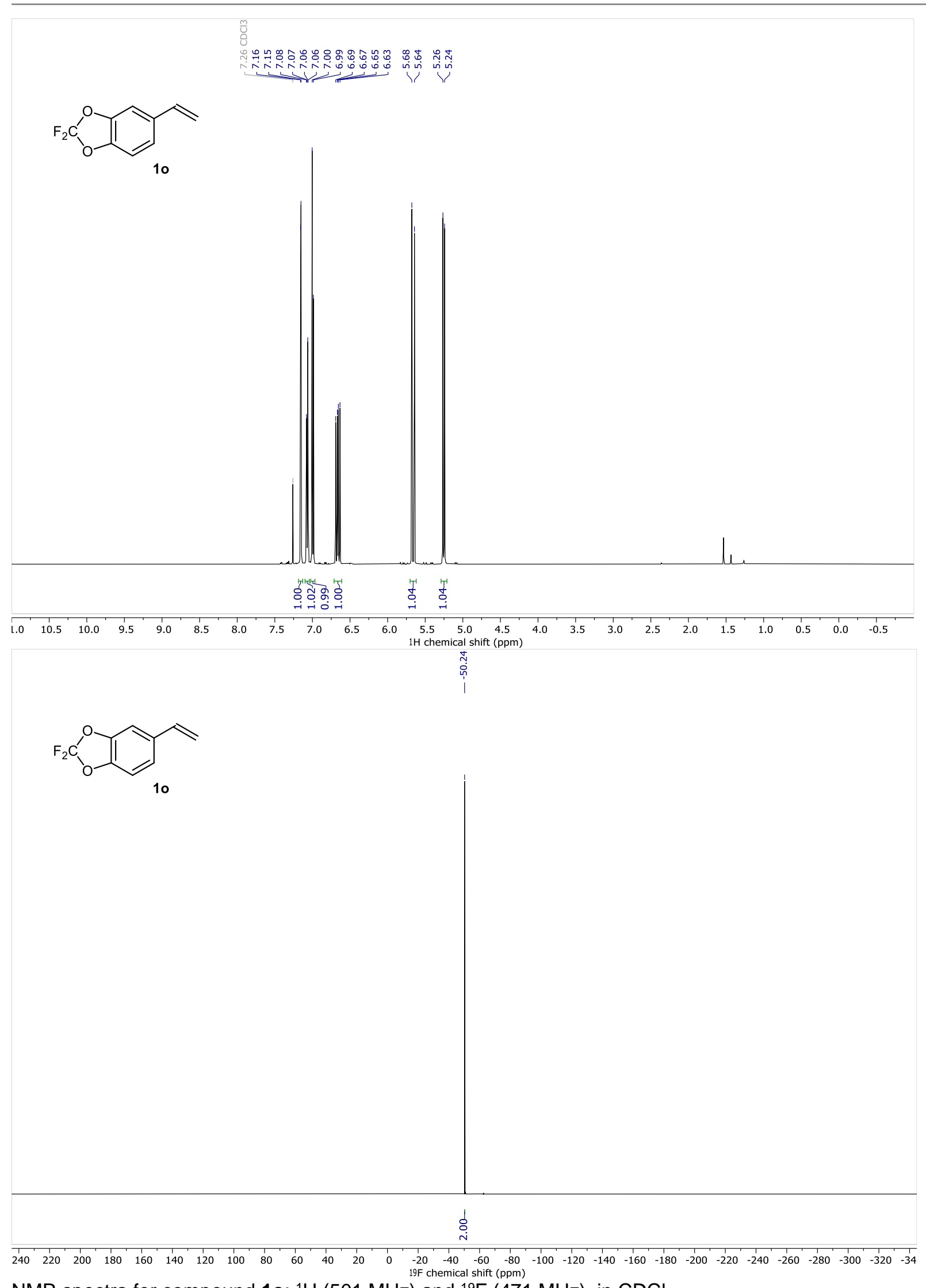

NMR spectra for compound 10: ${ }^{1} \mathrm{H}(501 \mathrm{MHz})$ and ${ }^{19} \mathrm{~F}(471 \mathrm{MHz})$, in $\mathrm{CDCl}_{3}$. 


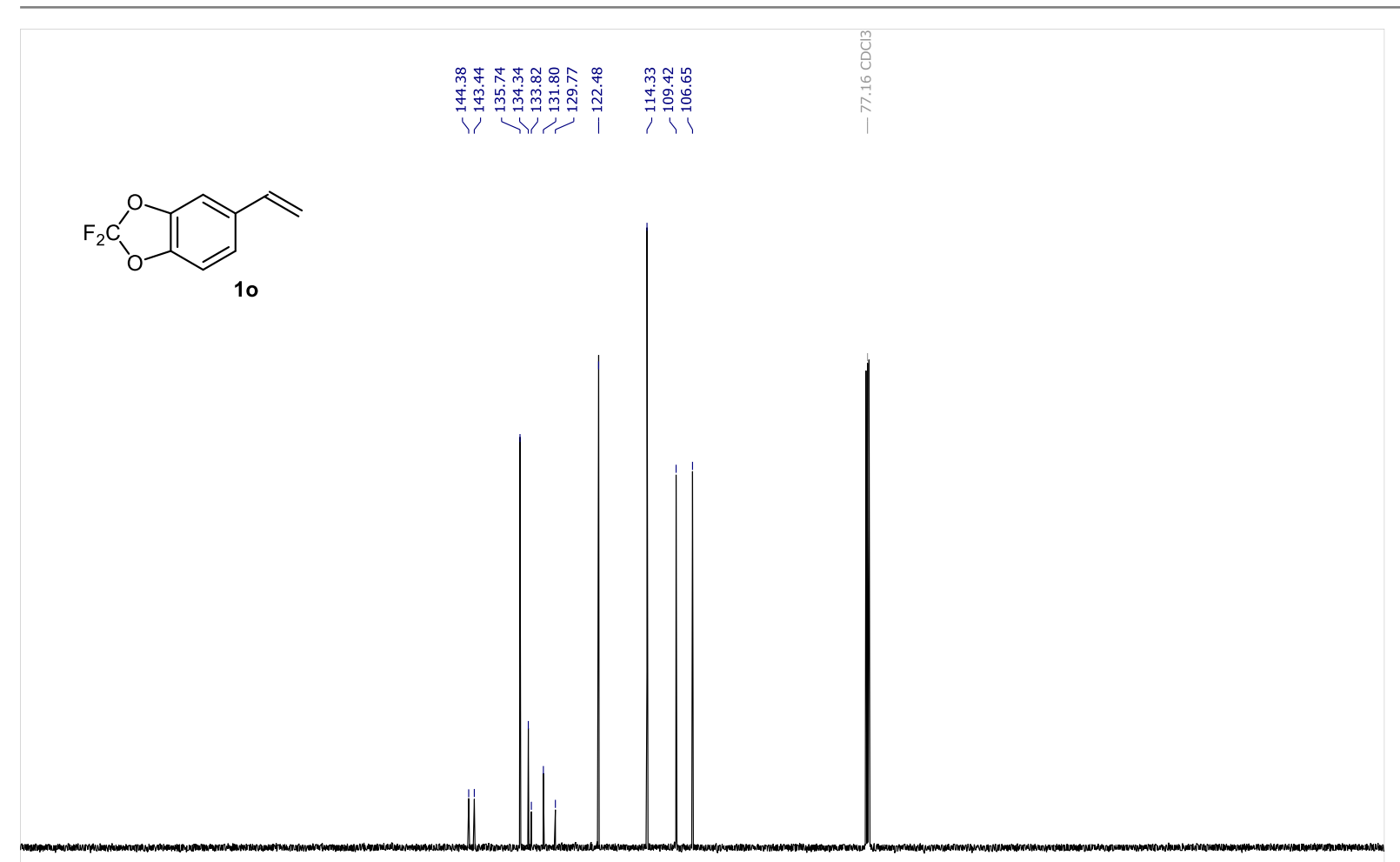

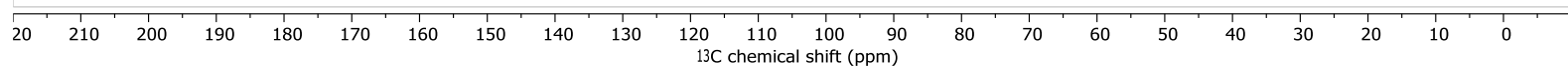

NMR spectra for compound 10 (continuation): ${ }^{13} \mathrm{C}(126 \mathrm{MHz})$, in $\mathrm{CDCl}_{3}$. 

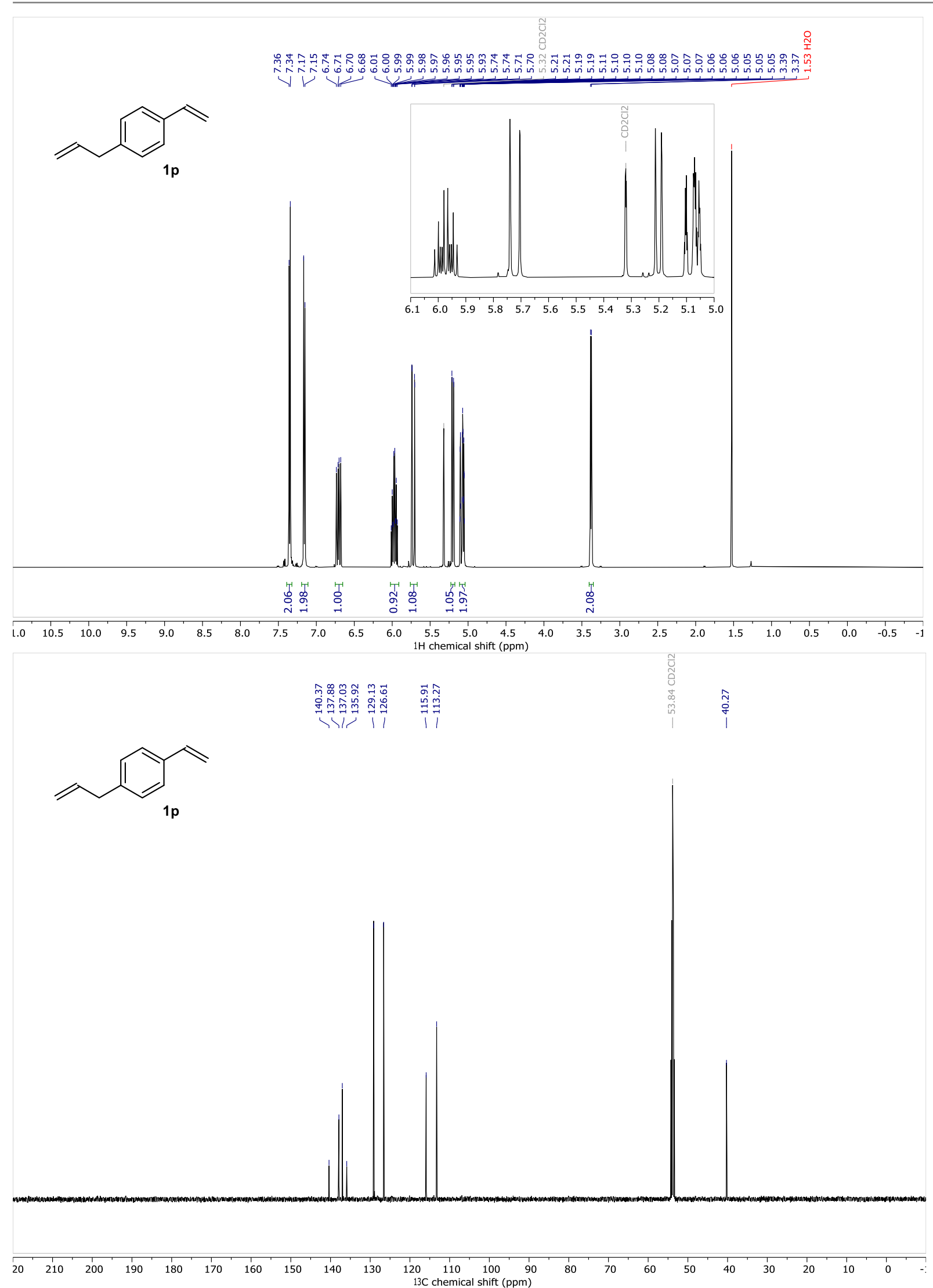

NMR spectra for compound $1 \mathrm{p}:{ }^{1} \mathrm{H}(501 \mathrm{MHz})$ and ${ }^{13} \mathrm{C}(126 \mathrm{MHz})$, in $\mathrm{CD}_{2} \mathrm{Cl}_{2}$ 
The Catalytic Asymmetric Intermolecular Prins Reaction

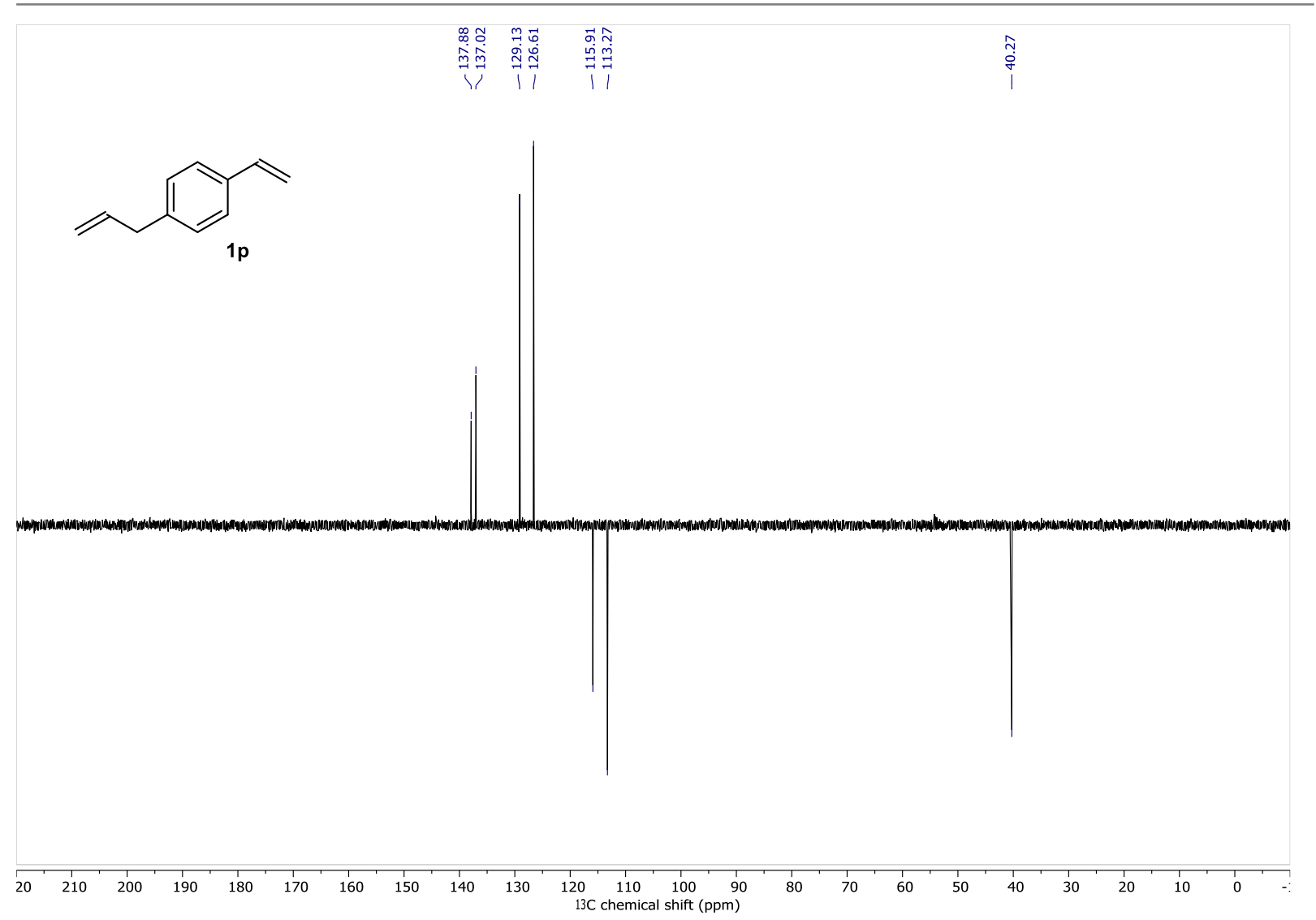

NMR spectra for compound $\mathbf{1 p}$ (continuation): DEPT-135 (126 MHz), in $\mathrm{CD}_{2} \mathrm{Cl}_{2}$. 


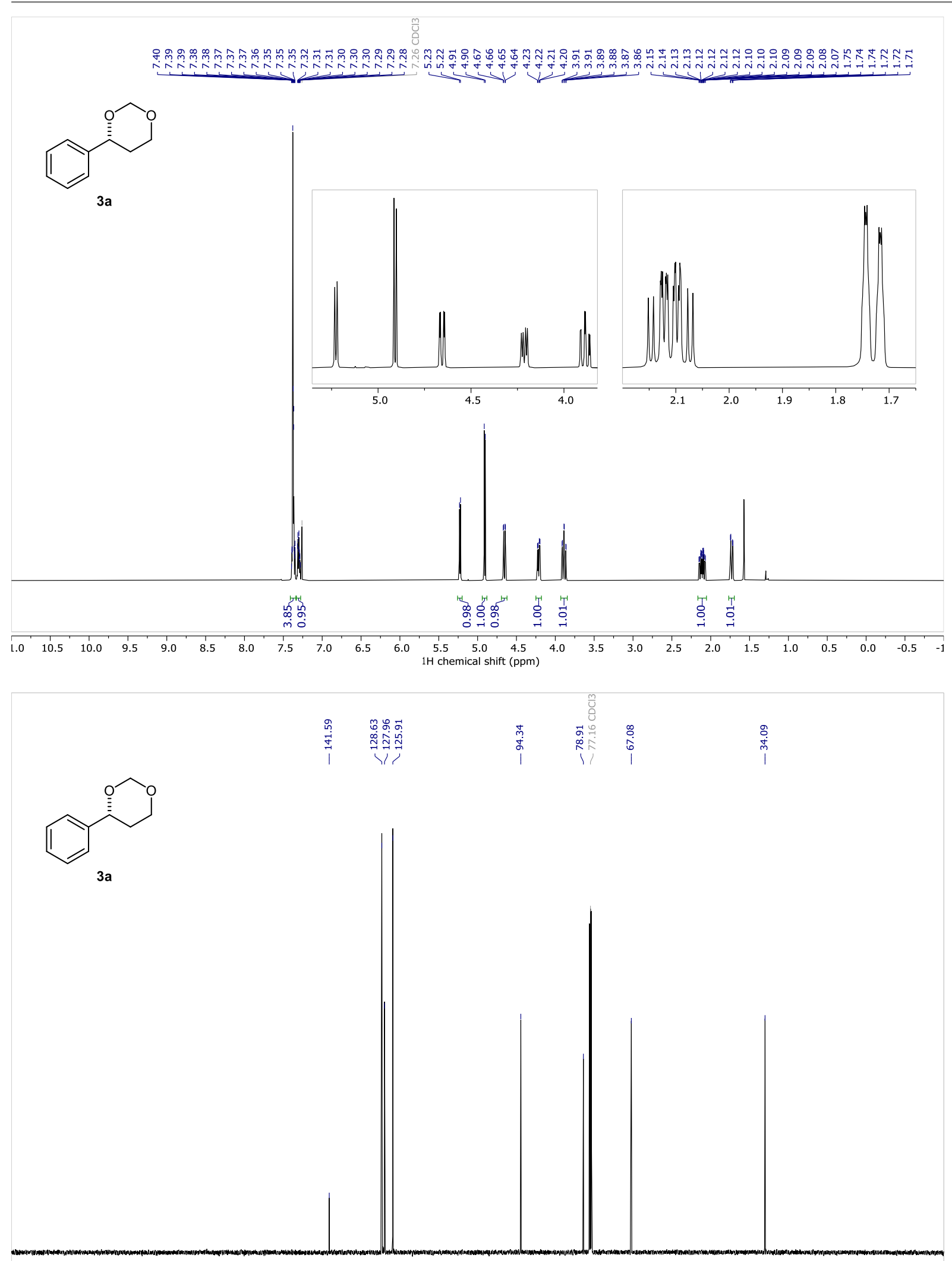

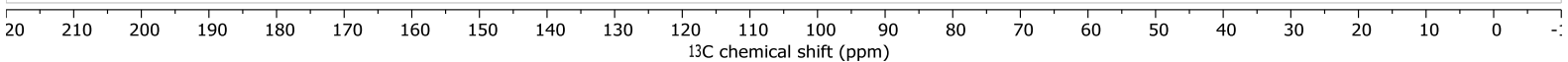

NMR spectra for compound 3a: ${ }^{1} \mathrm{H}(501 \mathrm{MHz})$ and ${ }^{13} \mathrm{C}(126 \mathrm{MHz})$, in $\mathrm{CDCl}_{3}$. 


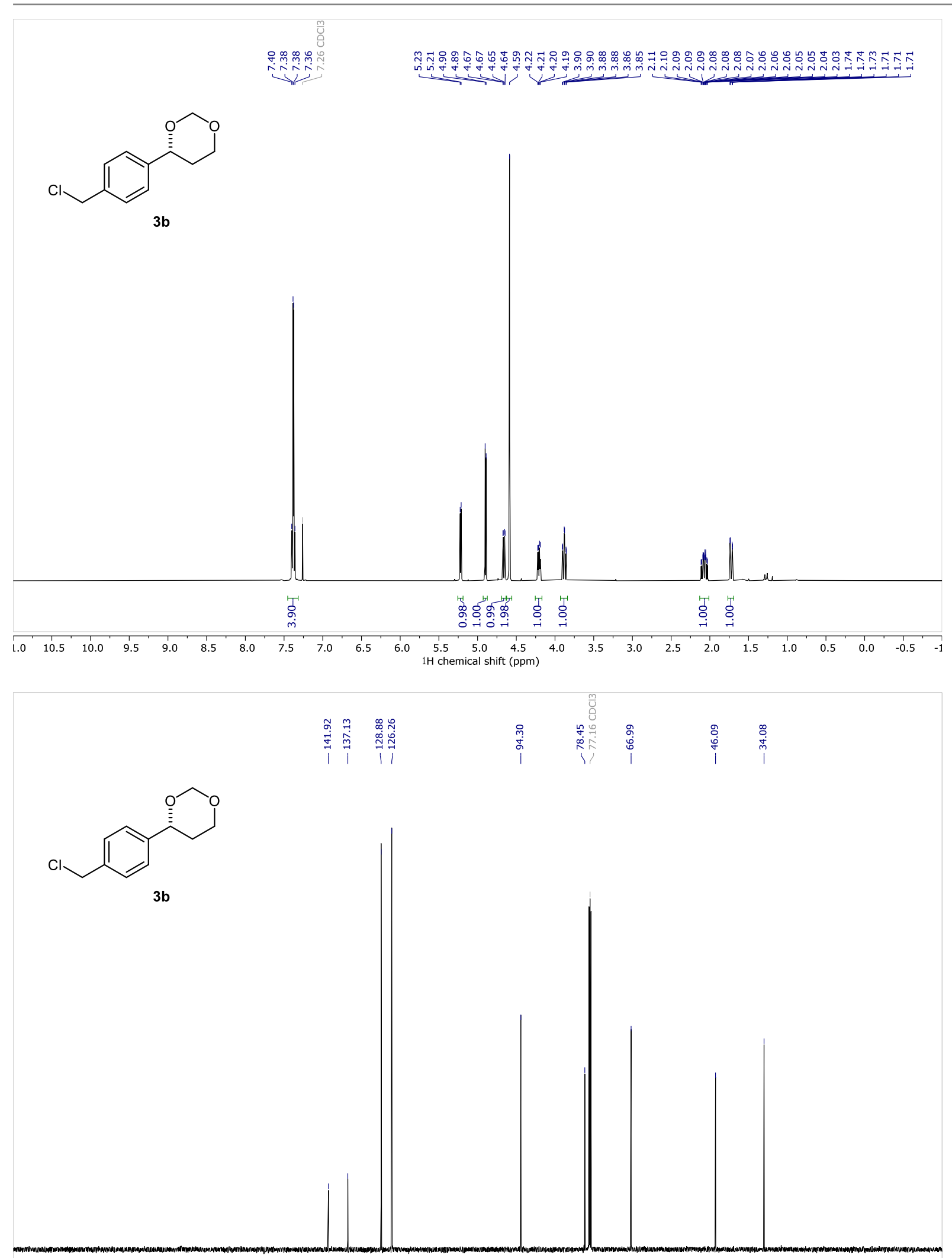

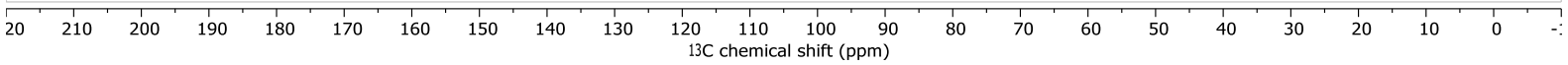

NMR spectra for compound $\mathbf{3 b}:{ }^{1} \mathrm{H}(501 \mathrm{MHz})$ and ${ }^{13} \mathrm{C}(126 \mathrm{MHz})$, in $\mathrm{CDCl}_{3}$. 

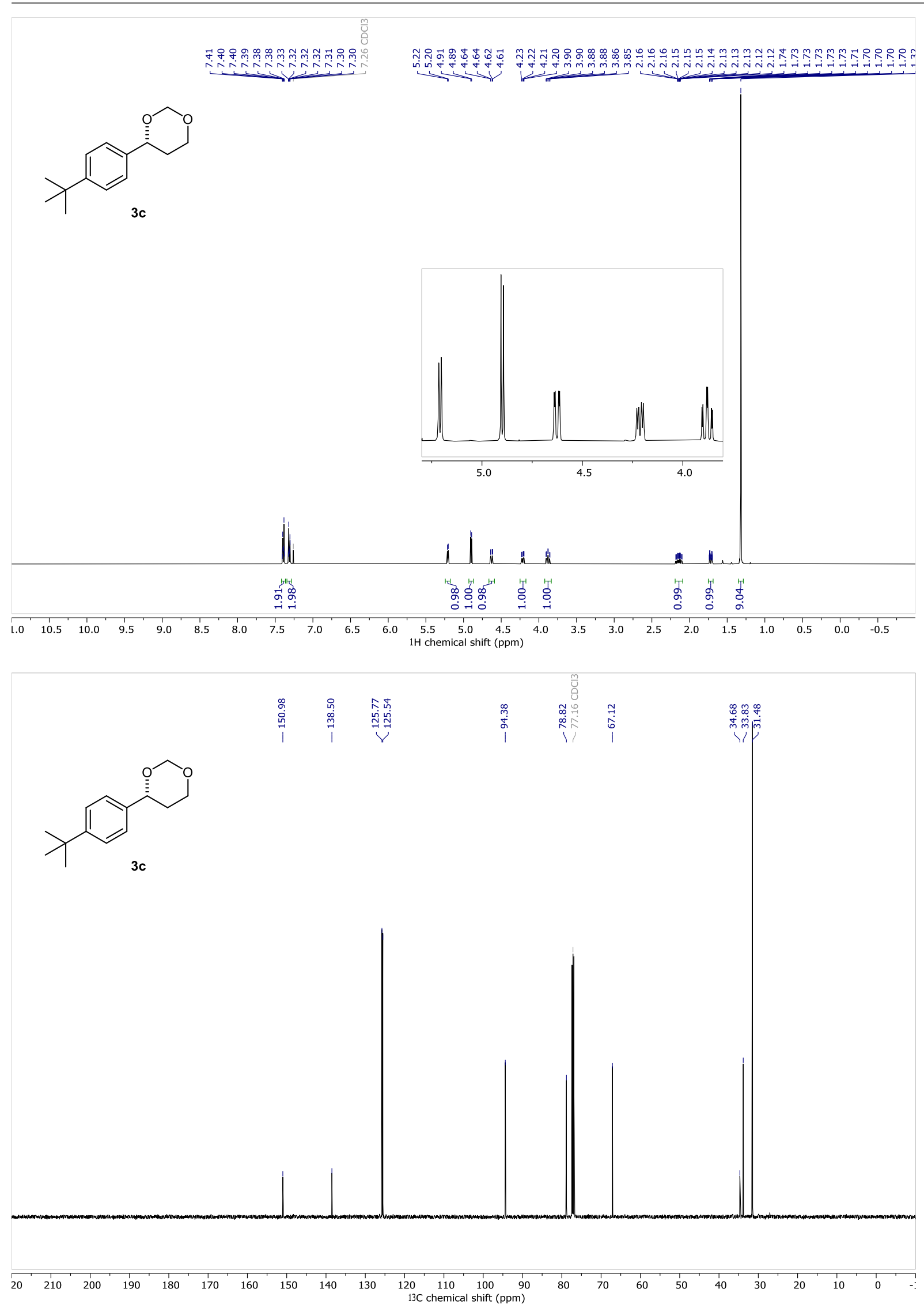

NMR spectra for compound 3c: ${ }^{1} \mathrm{H}(501 \mathrm{MHz})$ and ${ }^{13} \mathrm{C}(126 \mathrm{MHz})$, in $\mathrm{CDCl}_{3}$. 

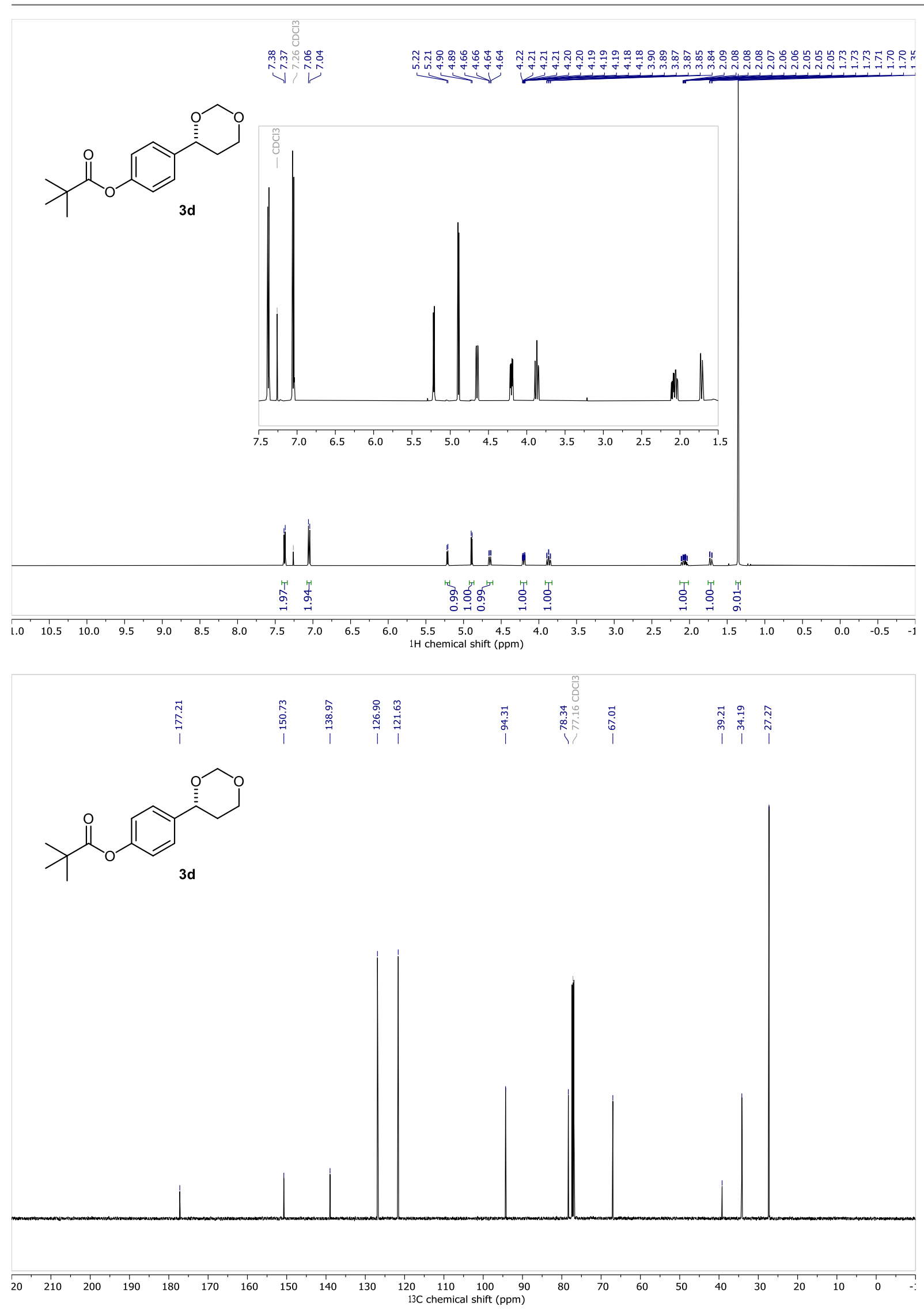

NMR spectra for compound 3d: ${ }^{1} \mathrm{H}(501 \mathrm{MHz})$ and ${ }^{13} \mathrm{C}(126 \mathrm{MHz})$, in $\mathrm{CDCl}_{3}$. 

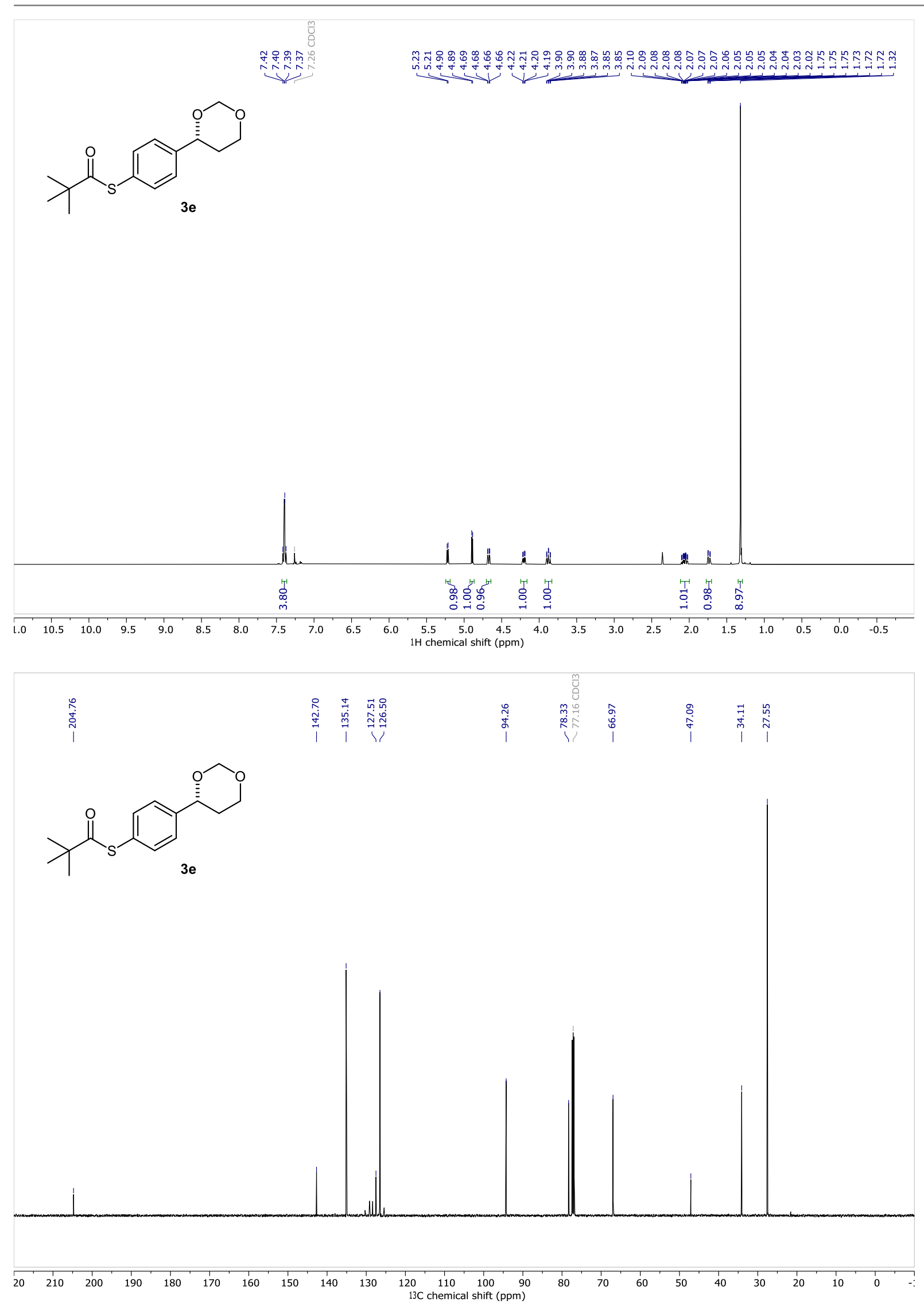

NMR spectra for compound $3 e:{ }^{1} \mathrm{H}(501 \mathrm{MHz})$ and ${ }^{13} \mathrm{C}(126 \mathrm{MHz})$, in $\mathrm{CDCl}_{3}$. 


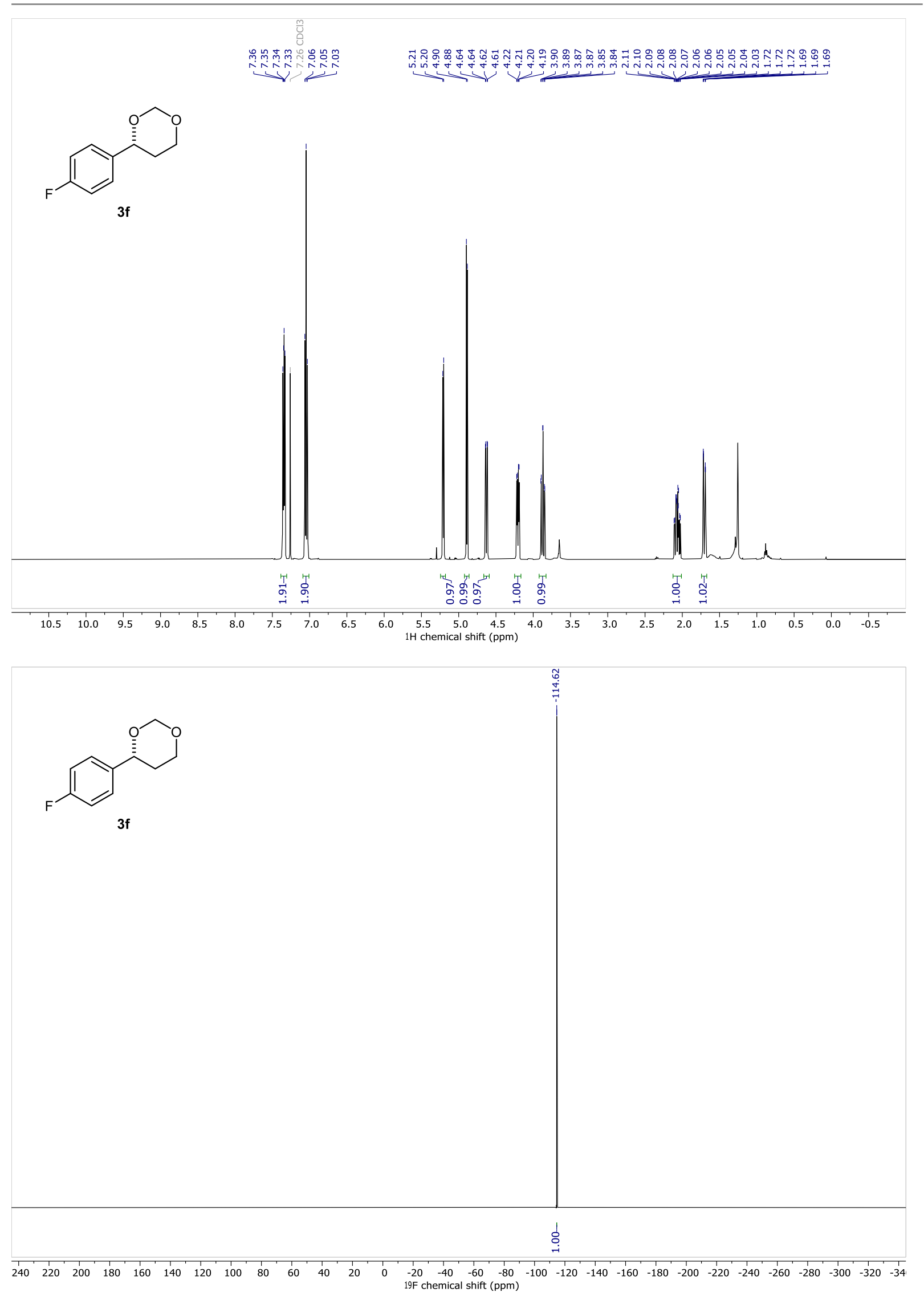

NMR spectra for compound 3f: ${ }^{1} \mathrm{H}(501 \mathrm{MHz})$ and ${ }^{19} \mathrm{~F}(471 \mathrm{MHz})$, in $\mathrm{CDCl}_{3}$. 


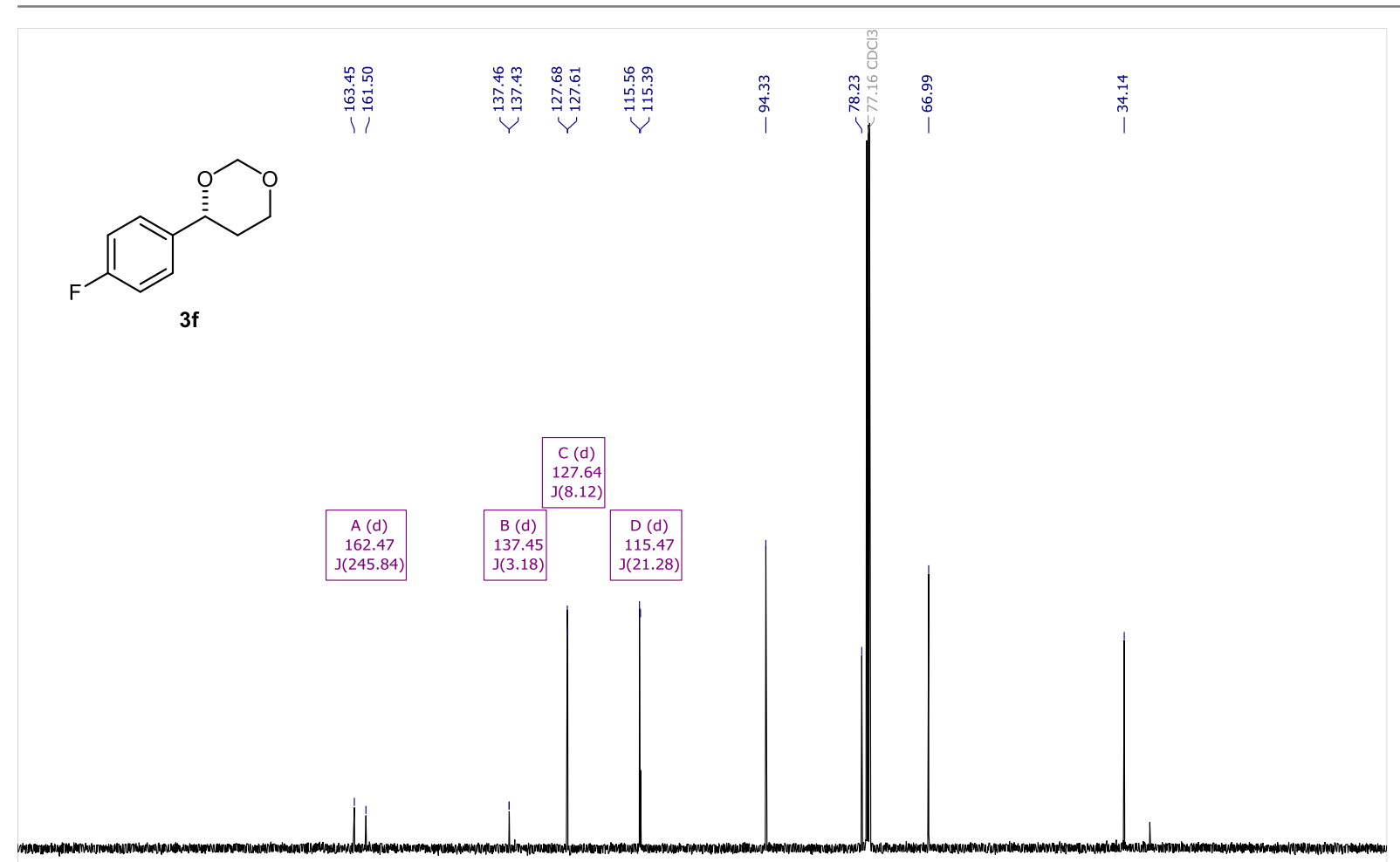

$\begin{array}{llllllllllll}210 & 200 & 190 & 180 & 170 & 160 & 150 & 140 & 130 & 120 & 110 & 100 \\ 13 \mathrm{C} \text { chemical shift (ppm) }\end{array}$

NMR spectra for compound $\mathbf{3 f}$ (continuation): ${ }^{13} \mathrm{C}(126 \mathrm{MHz})$, in $\mathrm{CDCl}_{3}$. 


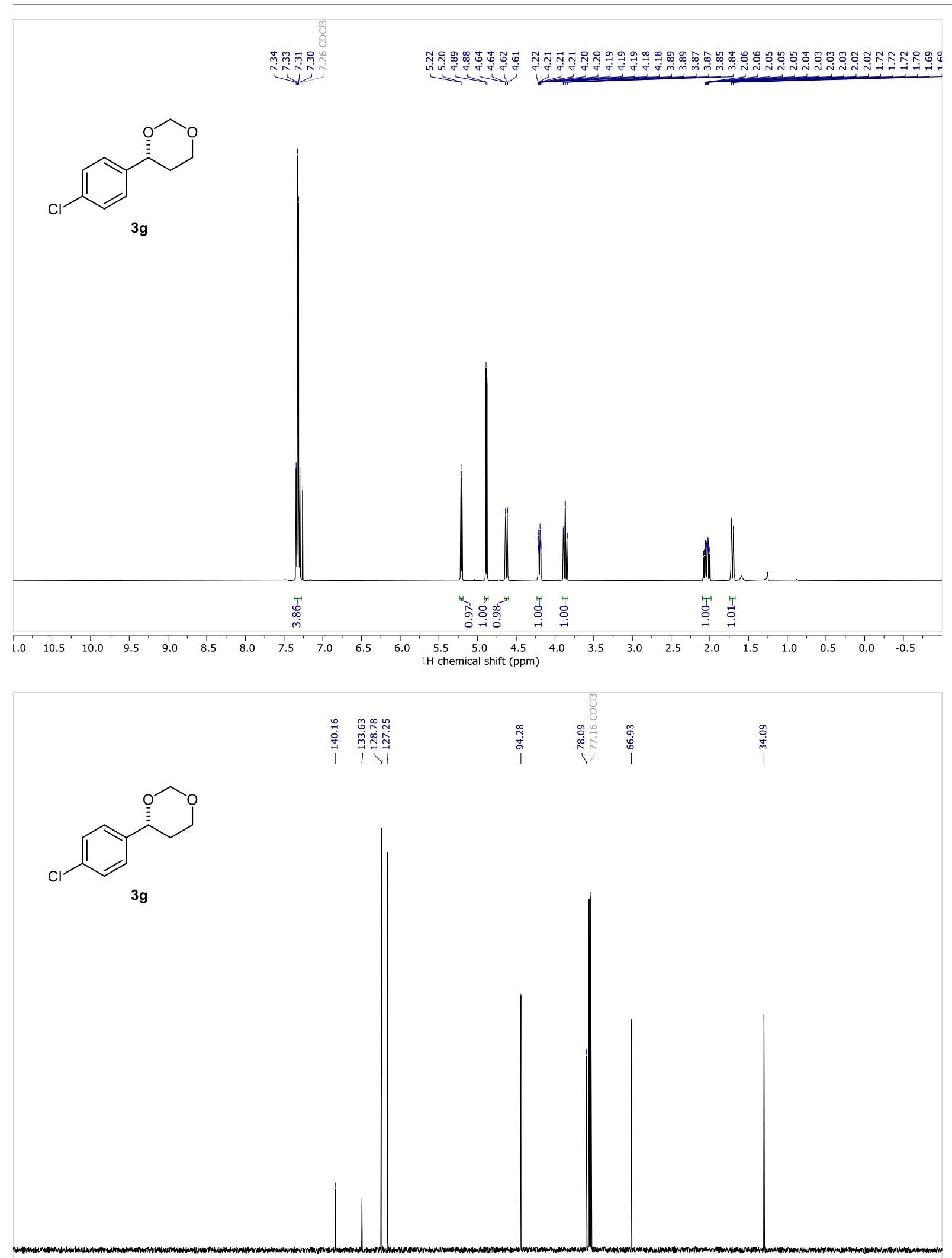

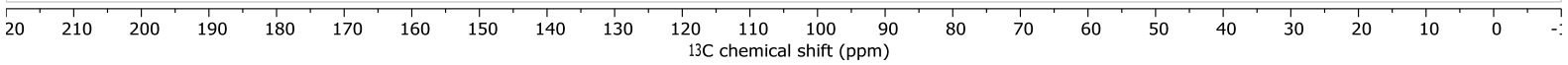

NMR spectra for compound $\mathbf{3 g}:{ }^{1} \mathrm{H}(501 \mathrm{MHz})$ and ${ }^{13} \mathrm{C}(126 \mathrm{MHz})$, in $\mathrm{CDCl}_{3}$. 


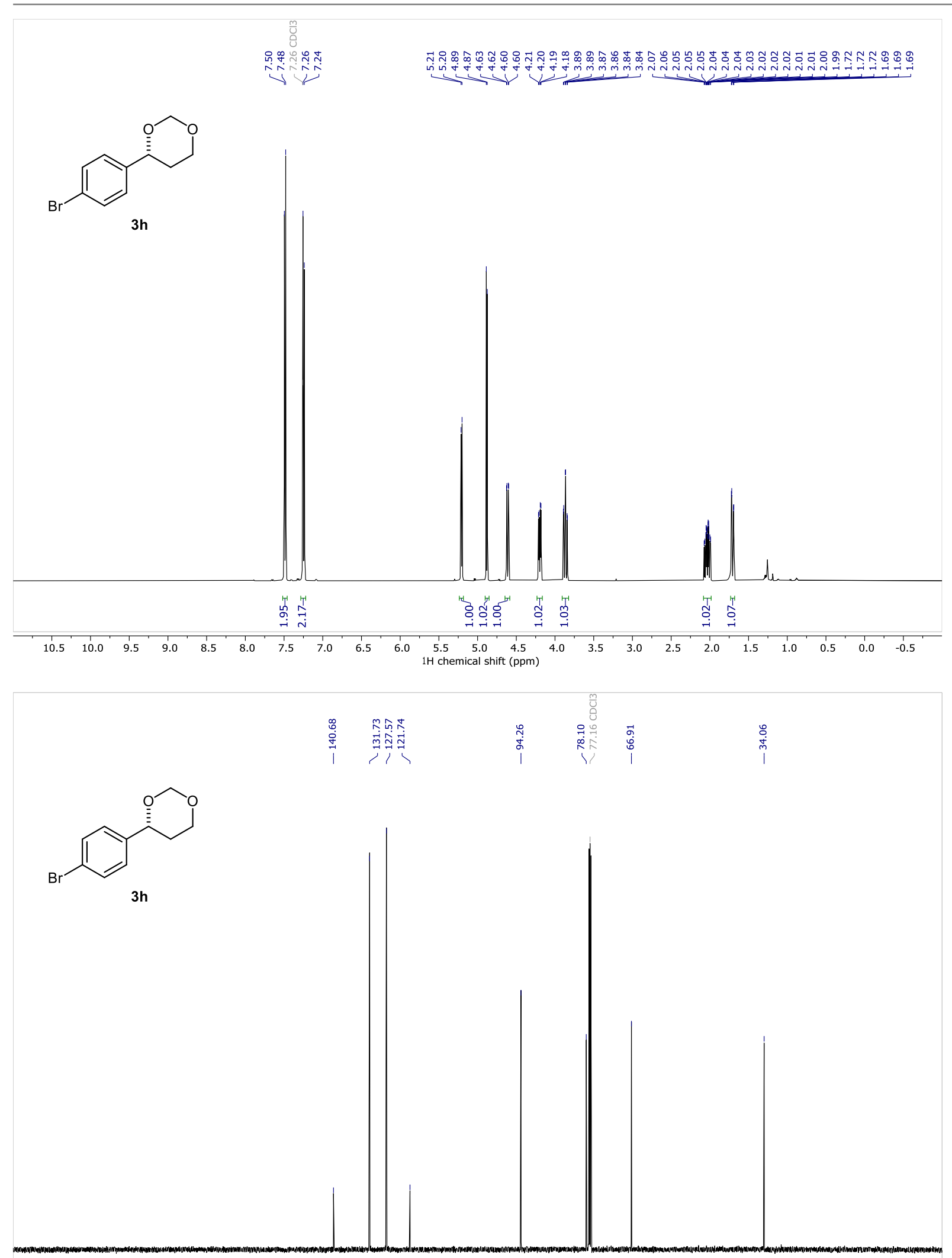

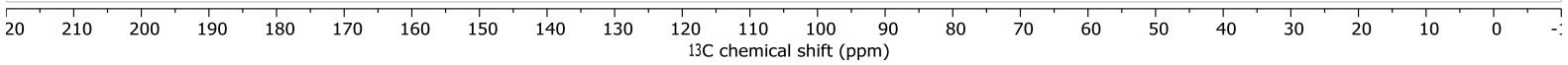

NMR spectra for compound 3h: ${ }^{1} \mathrm{H}(501 \mathrm{MHz})$ and ${ }^{13} \mathrm{C}(126 \mathrm{MHz})$, in $\mathrm{CDCl}_{3}$. 


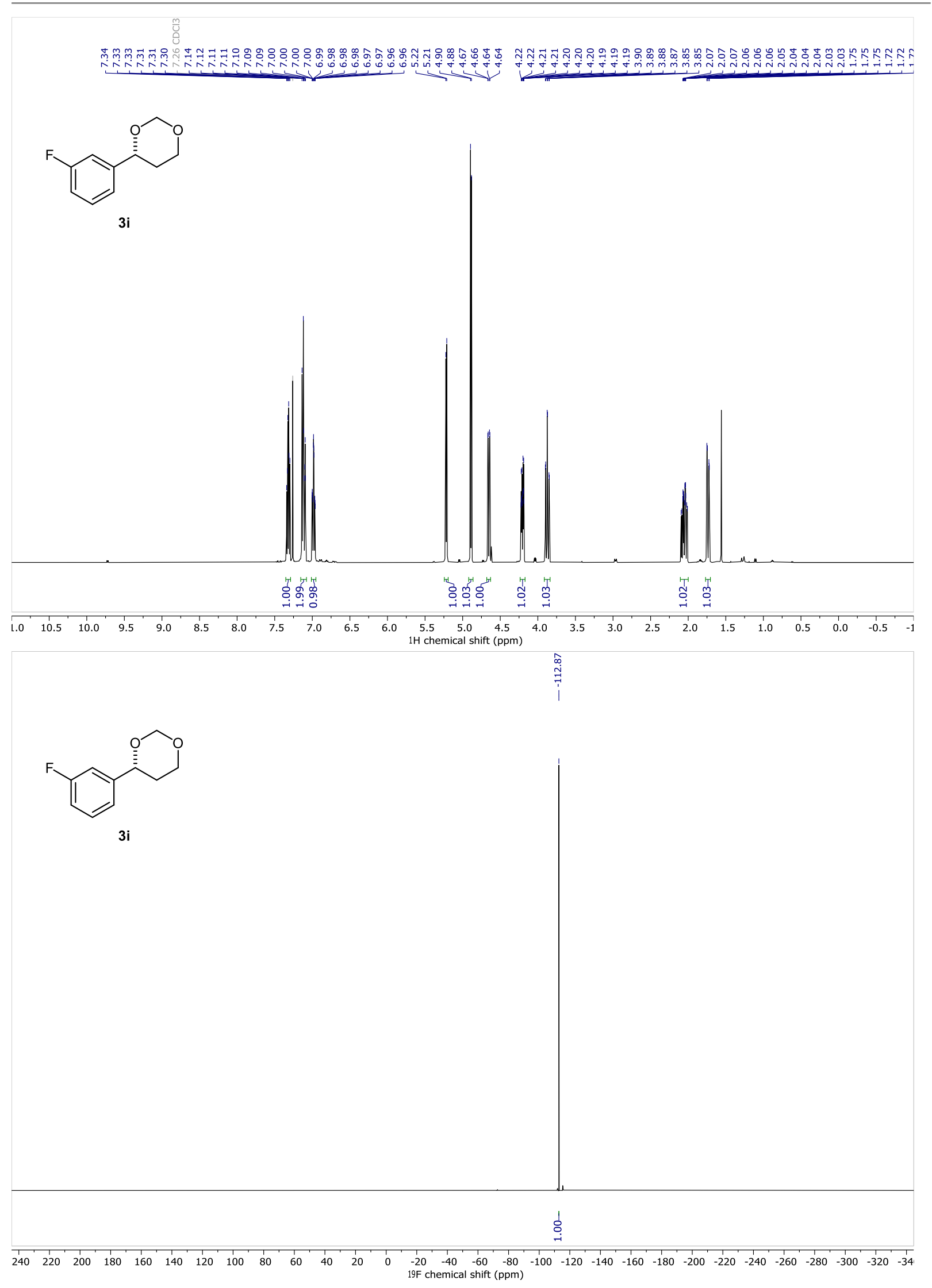

NMR spectra for compound 3i: ${ }^{1} \mathrm{H}(501 \mathrm{MHz})$ and ${ }^{19} \mathrm{~F}(471 \mathrm{MHz})$, in $\mathrm{CDCl}_{3}$. 


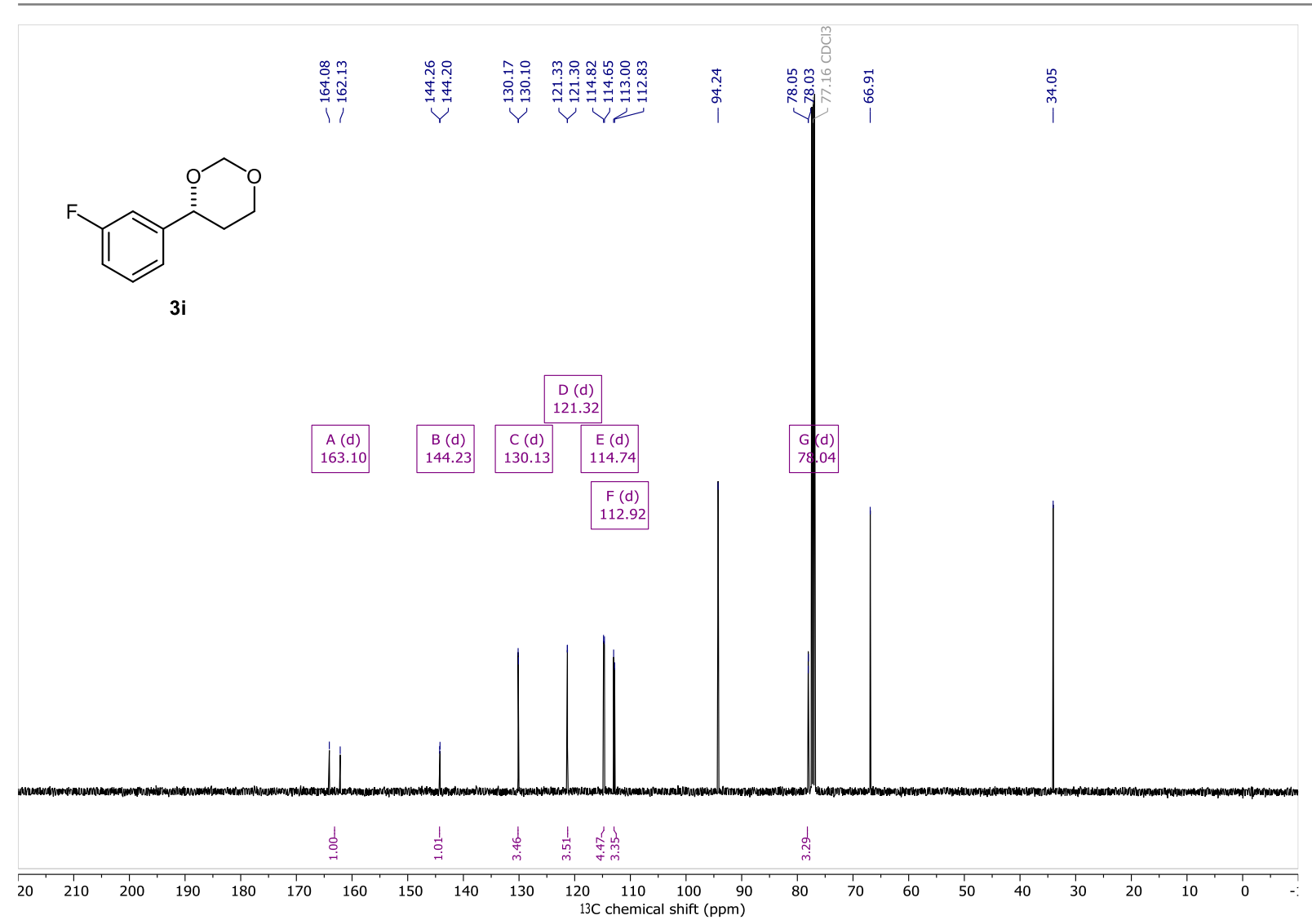

NMR spectra for compound $\mathbf{3 i}$ (continuation): ${ }^{13} \mathrm{C}$ (126 MHz), in $\mathrm{CDCl}_{3}$. 


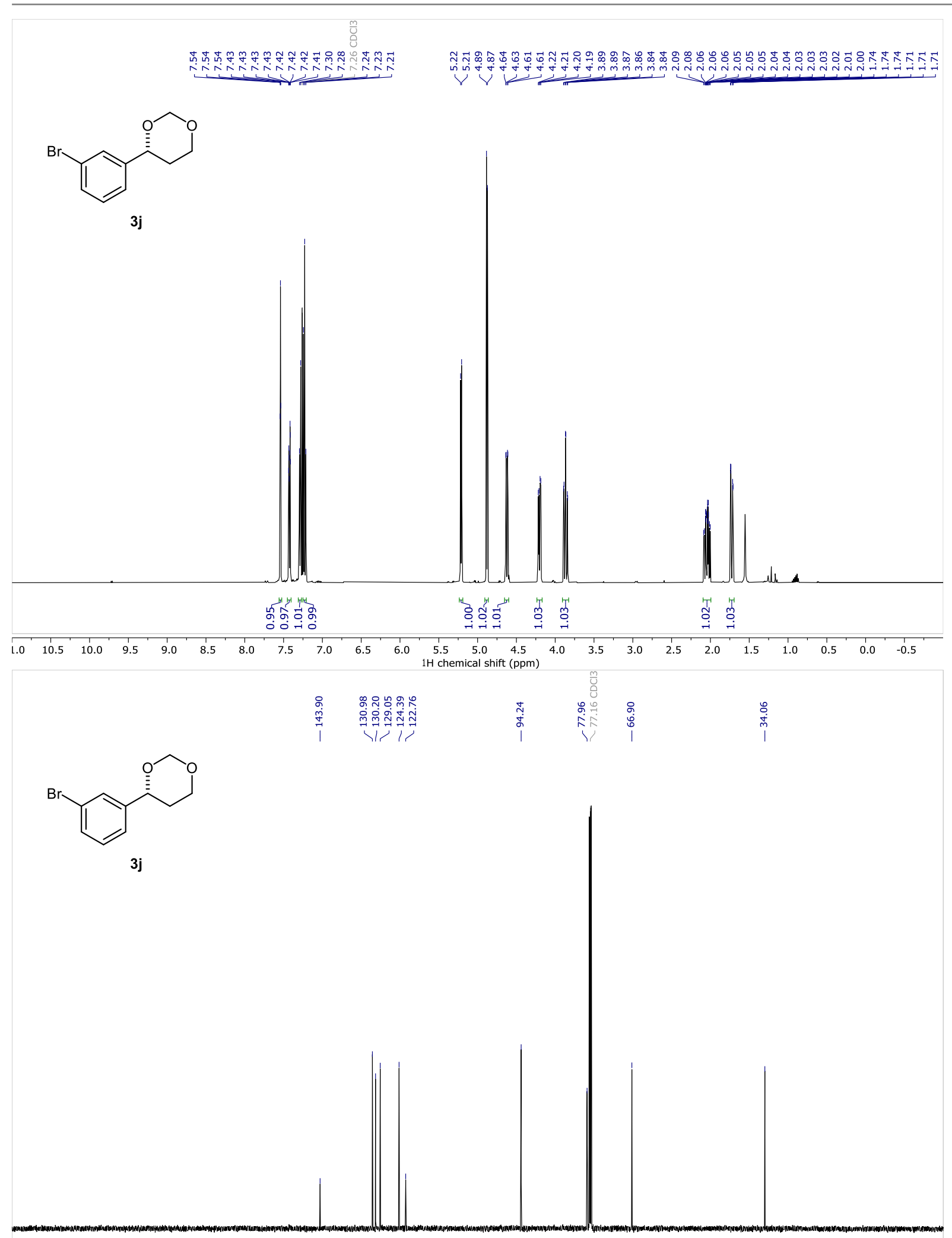

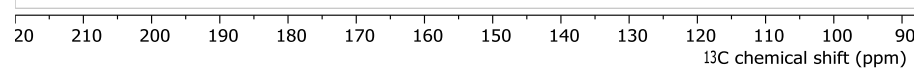

NMR spectra for compound 3j: ${ }^{1} \mathrm{H}(501 \mathrm{MHz})$ and ${ }^{13} \mathrm{C}(126 \mathrm{MHz})$, in $\mathrm{CDCl}_{3}$. 

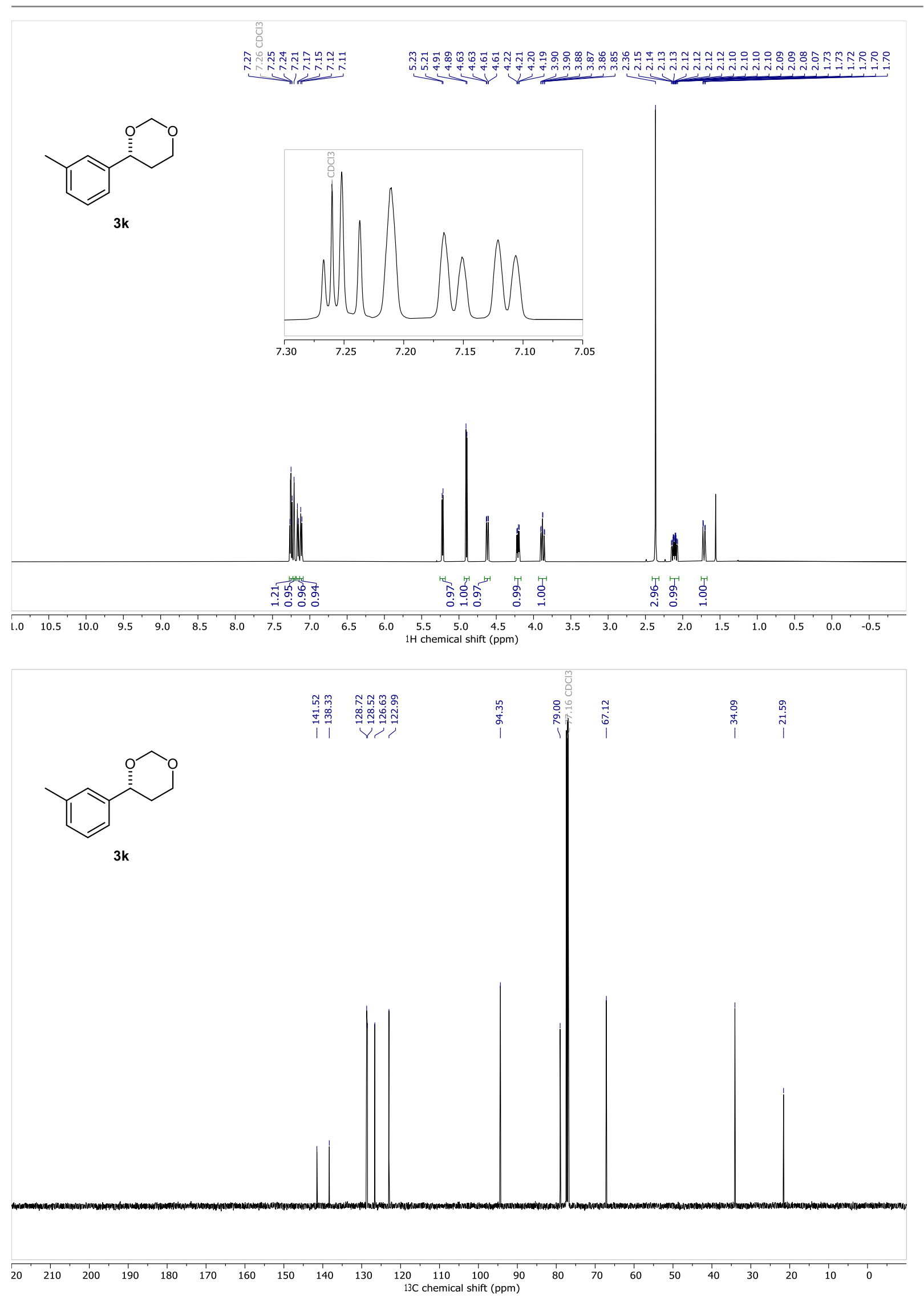

NMR spectra for compound $\mathbf{3 k}:{ }^{1} \mathrm{H}(501 \mathrm{MHz})$ and ${ }^{13} \mathrm{C}(126 \mathrm{MHz})$, in $\mathrm{CDCl}_{3}$. 


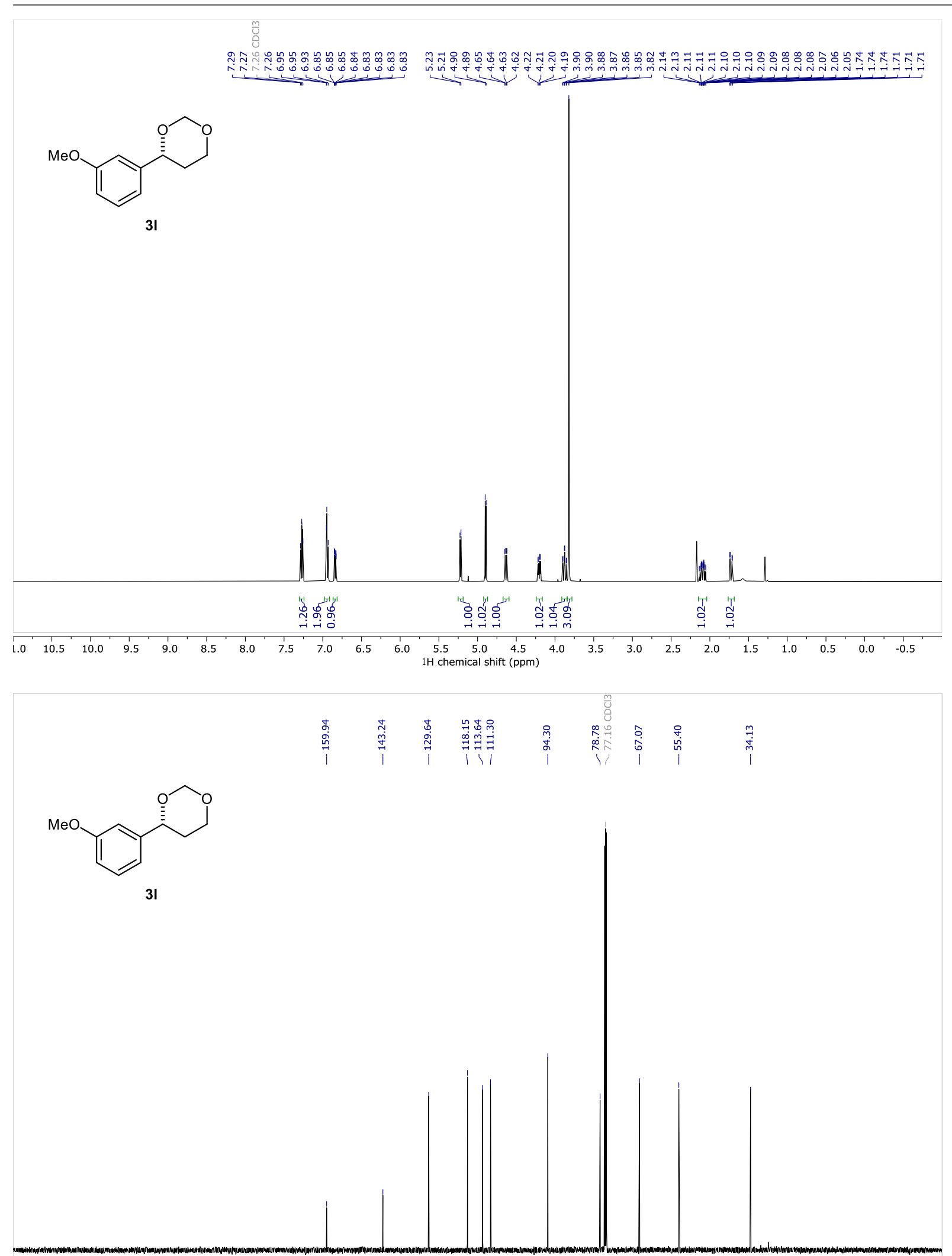

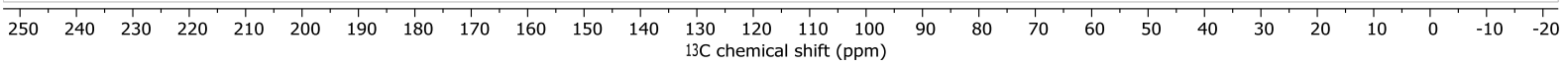

NMR spectra for compound 3I: ${ }^{1} \mathrm{H}(501 \mathrm{MHz})$ and ${ }^{13} \mathrm{C}(126 \mathrm{MHz})$, in $\mathrm{CDCl}_{3}$. 


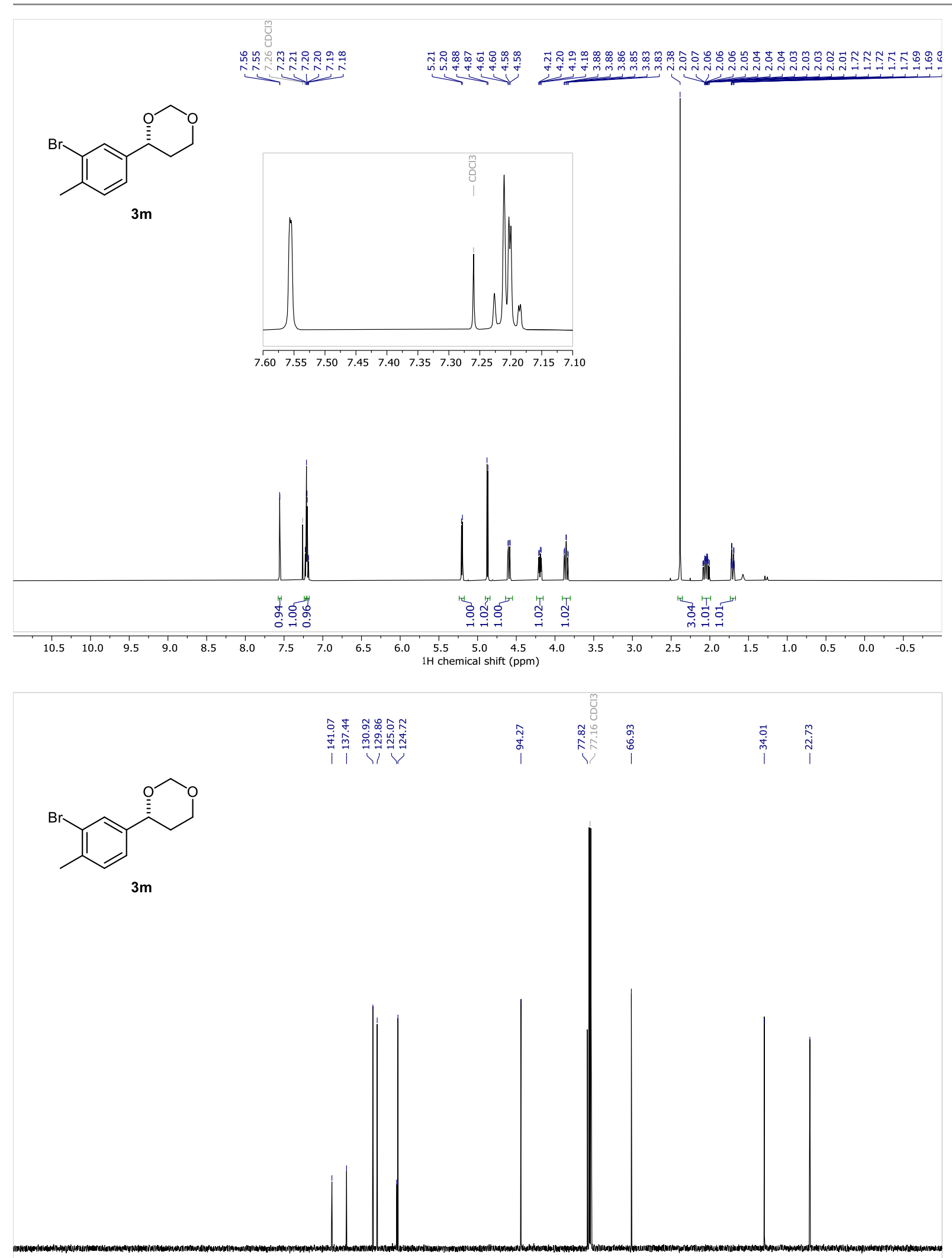

\begin{tabular}{lllllllllllllllllllllllllll}
\hline 20 & 210 & 200 & 190 & 180 & 170 & 160 & 150 & 140 & 130 & 120 & 110 & 100 & 90 & 80 & 70 & 60 & 50 & 40 & 30 & 20 & 10 & 0 & 130 \\
-
\end{tabular}

NMR spectra for compound $3 \mathrm{~m}:{ }^{1} \mathrm{H}(501 \mathrm{MHz})$ and ${ }^{13} \mathrm{C}(126 \mathrm{MHz})$, in $\mathrm{CDCl}_{3}$. 


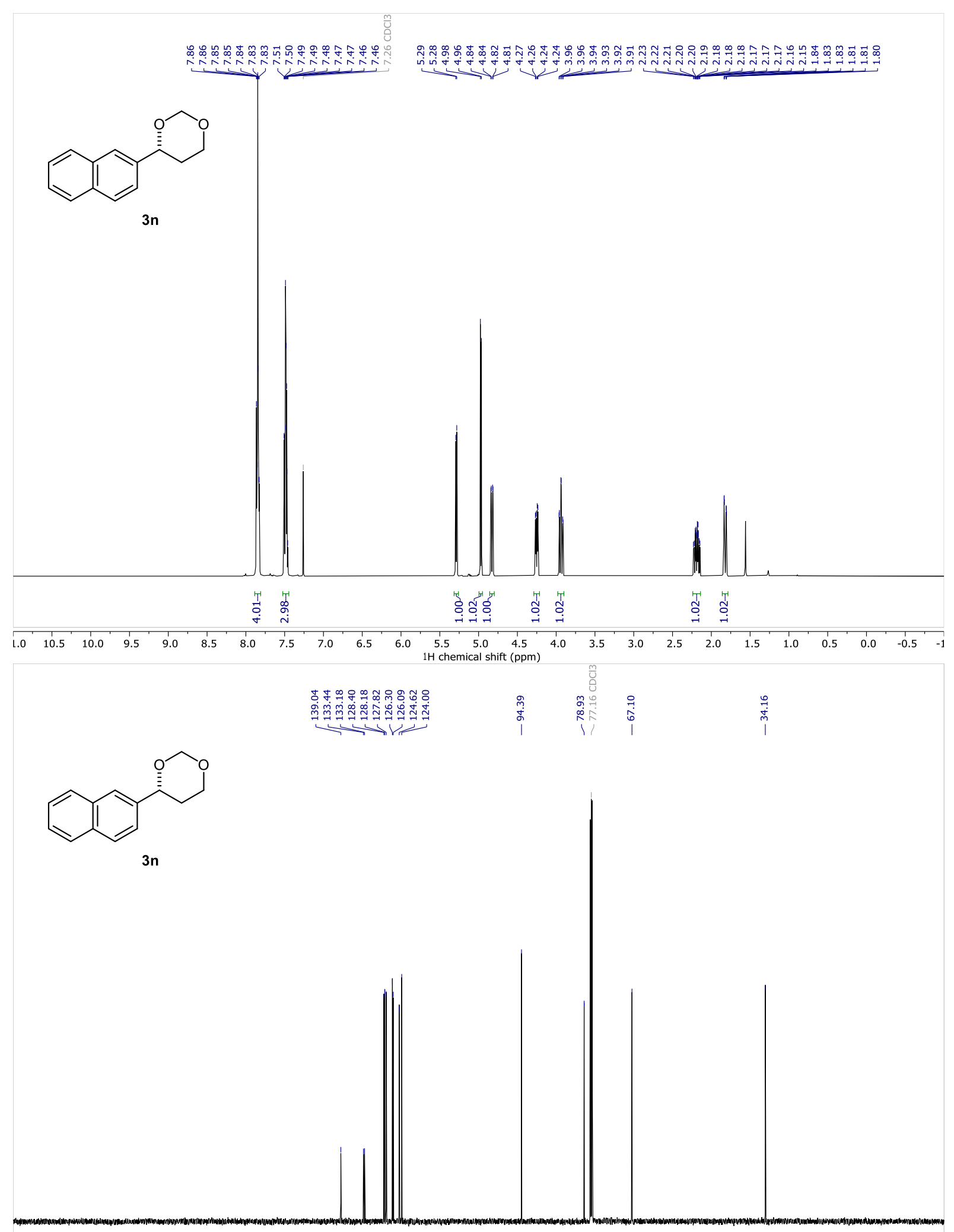

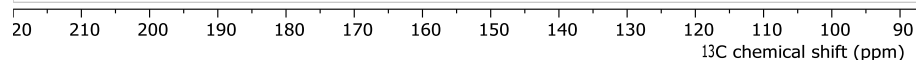

NMR spectra for compound 3n: ${ }^{1} \mathrm{H}(501 \mathrm{MHz})$ and ${ }^{13} \mathrm{C}(126 \mathrm{MHz})$, in $\mathrm{CDCl}_{3}$. 


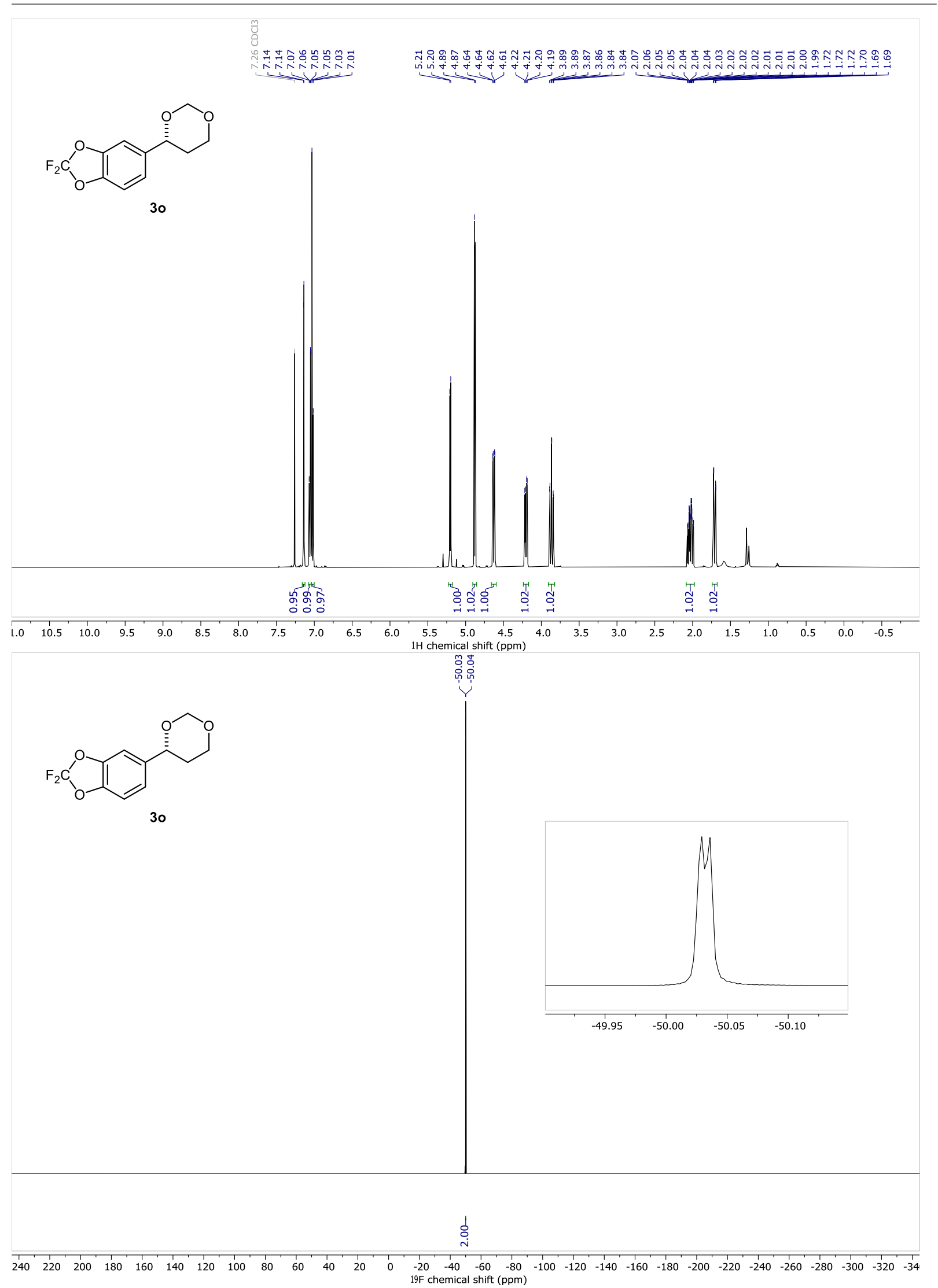

NMR spectra for compound $30:{ }^{1} \mathrm{H}(501 \mathrm{MHz})$ and ${ }^{19} \mathrm{~F}(471 \mathrm{MHz})$, in $\mathrm{CDCl}_{3}$. 


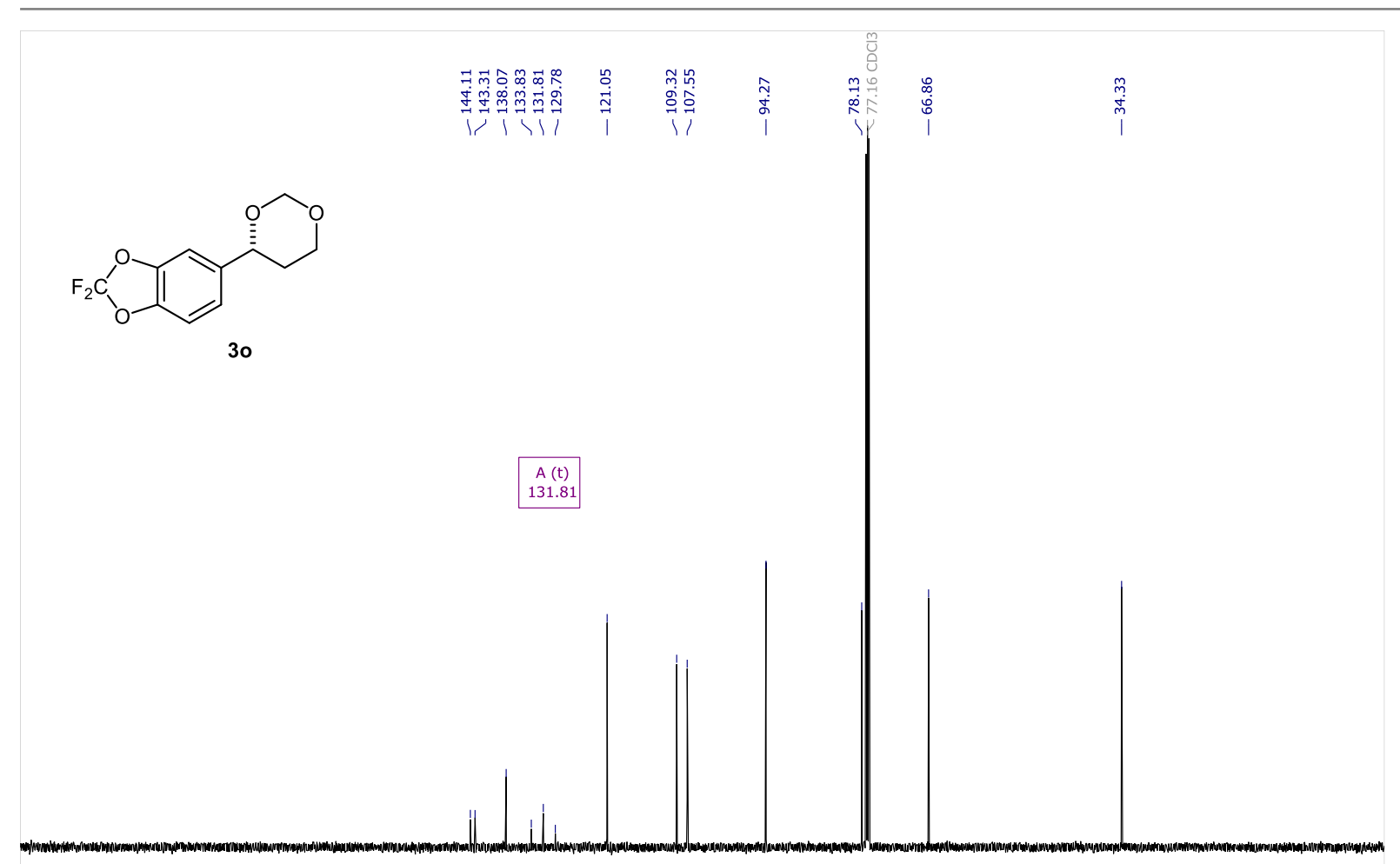

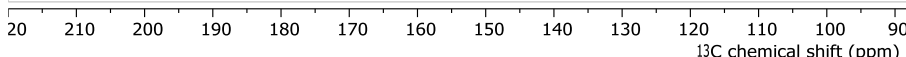

NMR spectra for compound 30 (continuation): ${ }^{13} \mathrm{C}(126 \mathrm{MHz})$, in $\mathrm{CDCl}_{3}$. 


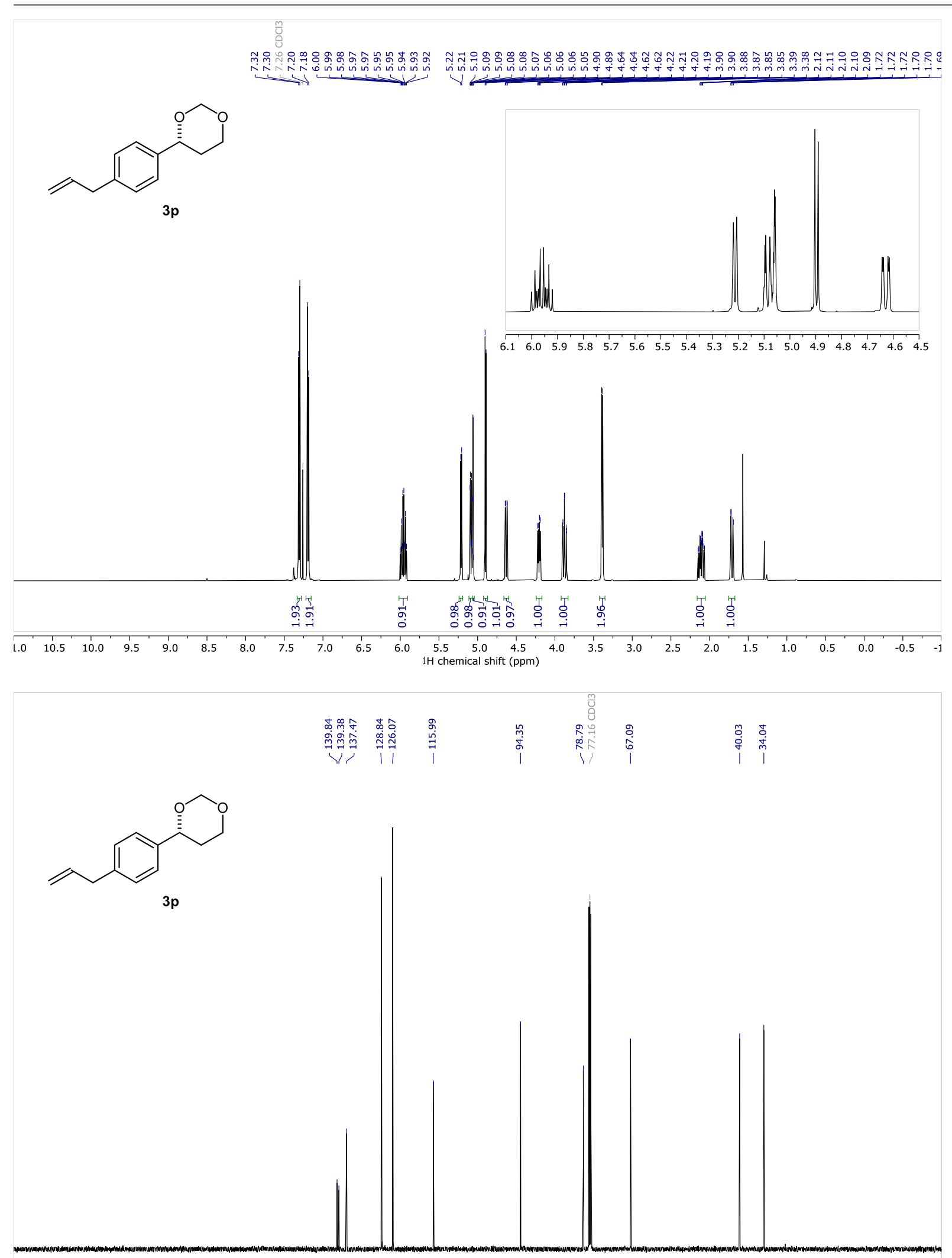

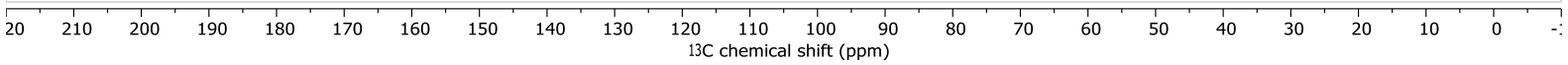

NMR spectra for compound 3p: ${ }^{1} \mathrm{H}(501 \mathrm{MHz})$ and ${ }^{13} \mathrm{C}(126 \mathrm{MHz})$, in $\mathrm{CDCl}_{3}$. 

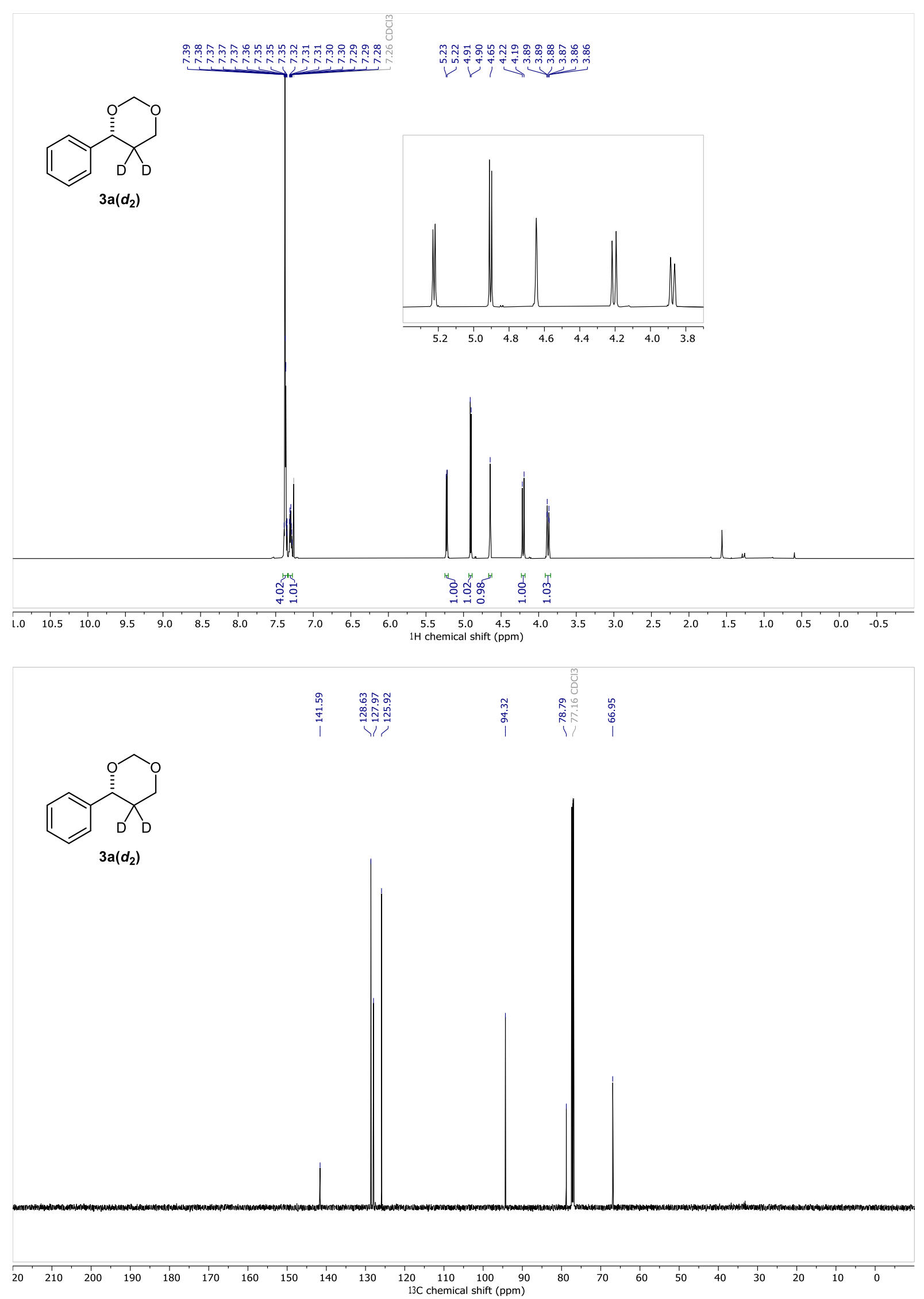

NMR spectra for compound $3 a\left(d_{2}\right):{ }^{1} \mathrm{H}(501 \mathrm{MHz})$ and ${ }^{13} \mathrm{C}(126 \mathrm{MHz})$, in $\mathrm{CDCl}_{3}$. 

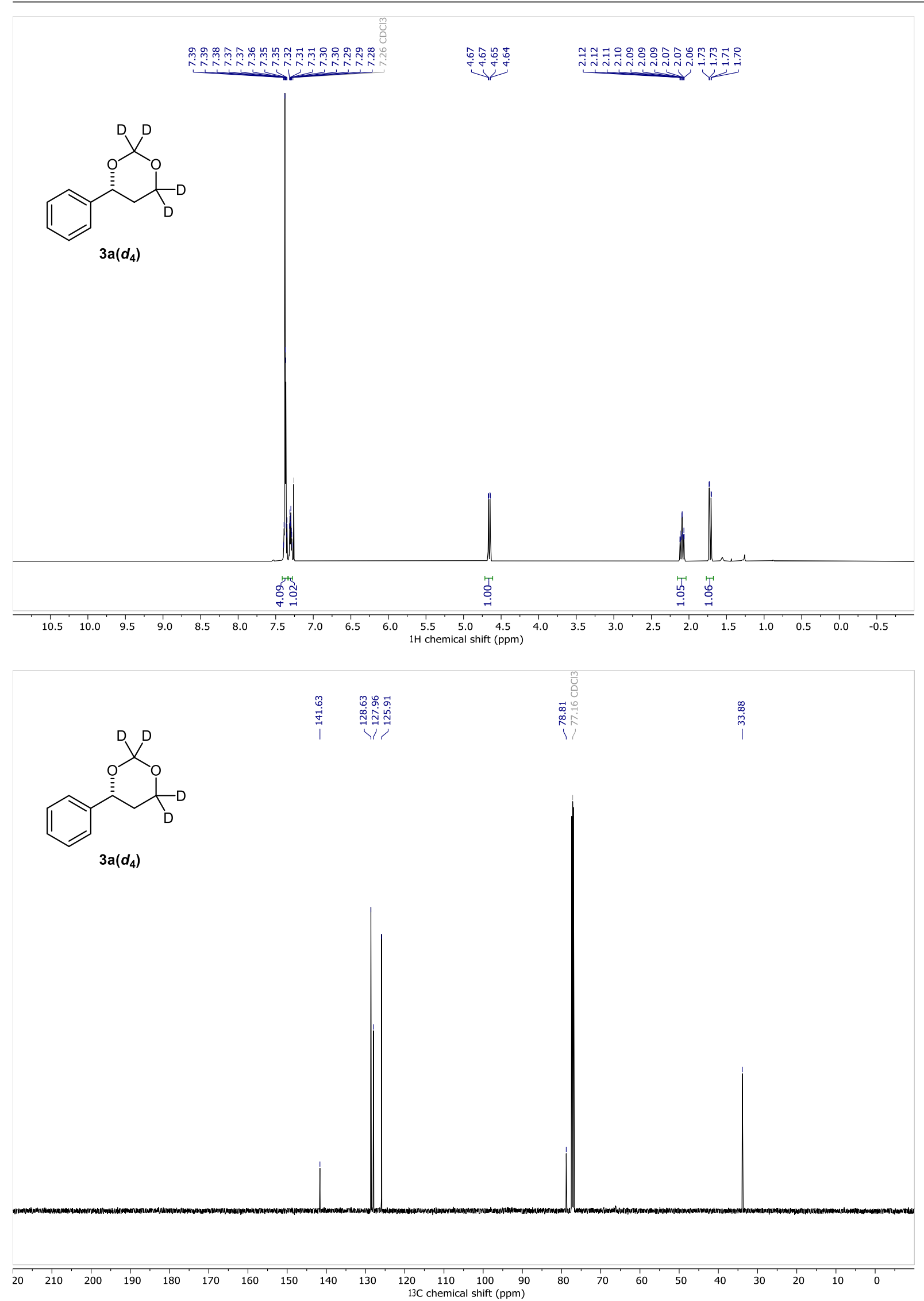

NMR spectra for compound $3 a\left(d_{4}\right):{ }^{1} \mathrm{H}(501 \mathrm{MHz})$ and ${ }^{13} \mathrm{C}(126 \mathrm{MHz})$, in $\mathrm{CDCl}_{3}$. 

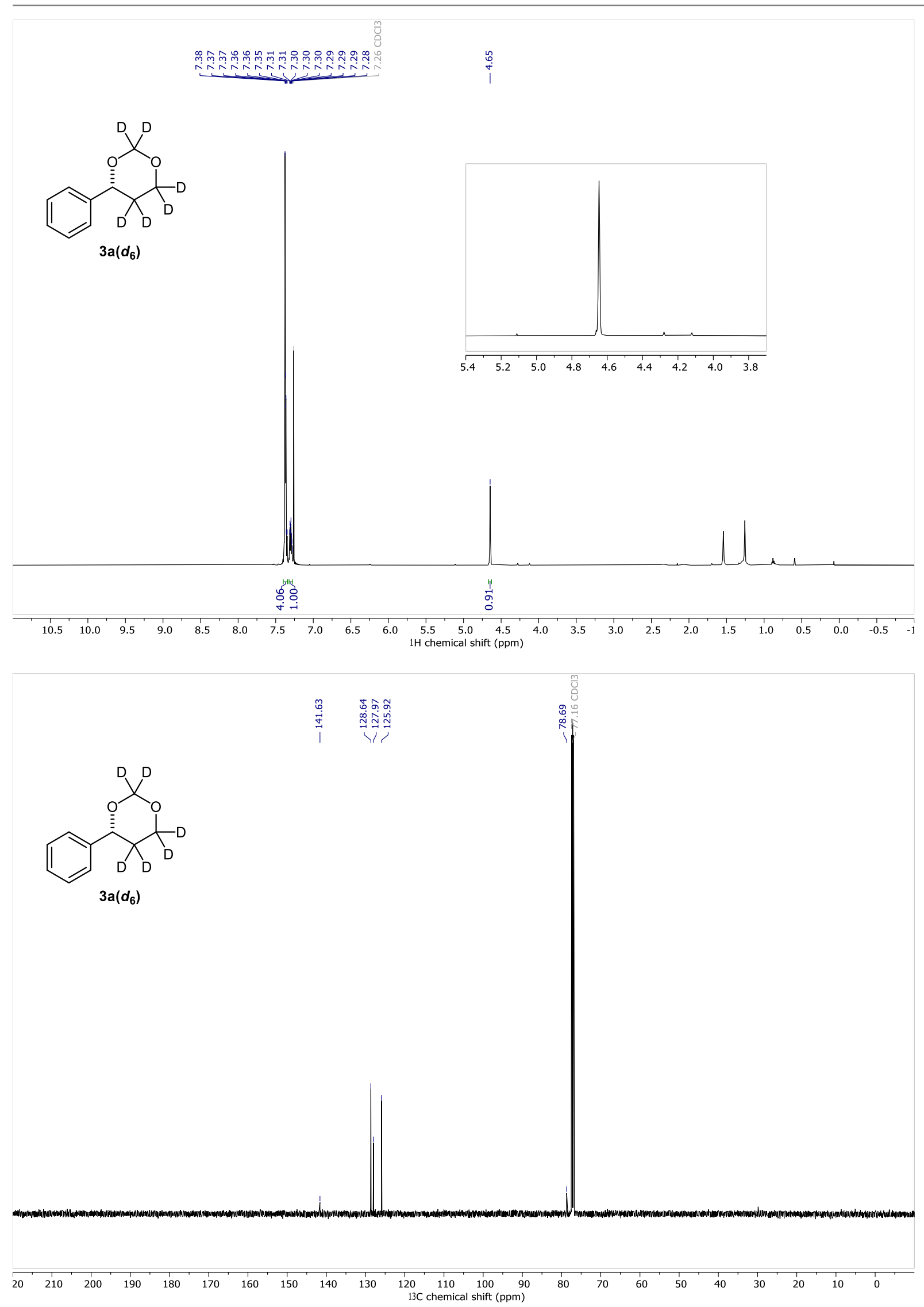

NMR spectra for compound $3 a\left(d_{6}\right):{ }^{1} \mathrm{H}(501 \mathrm{MHz})$ and ${ }^{13} \mathrm{C}(126 \mathrm{MHz})$, in $\mathrm{CDCl}_{3}$. 


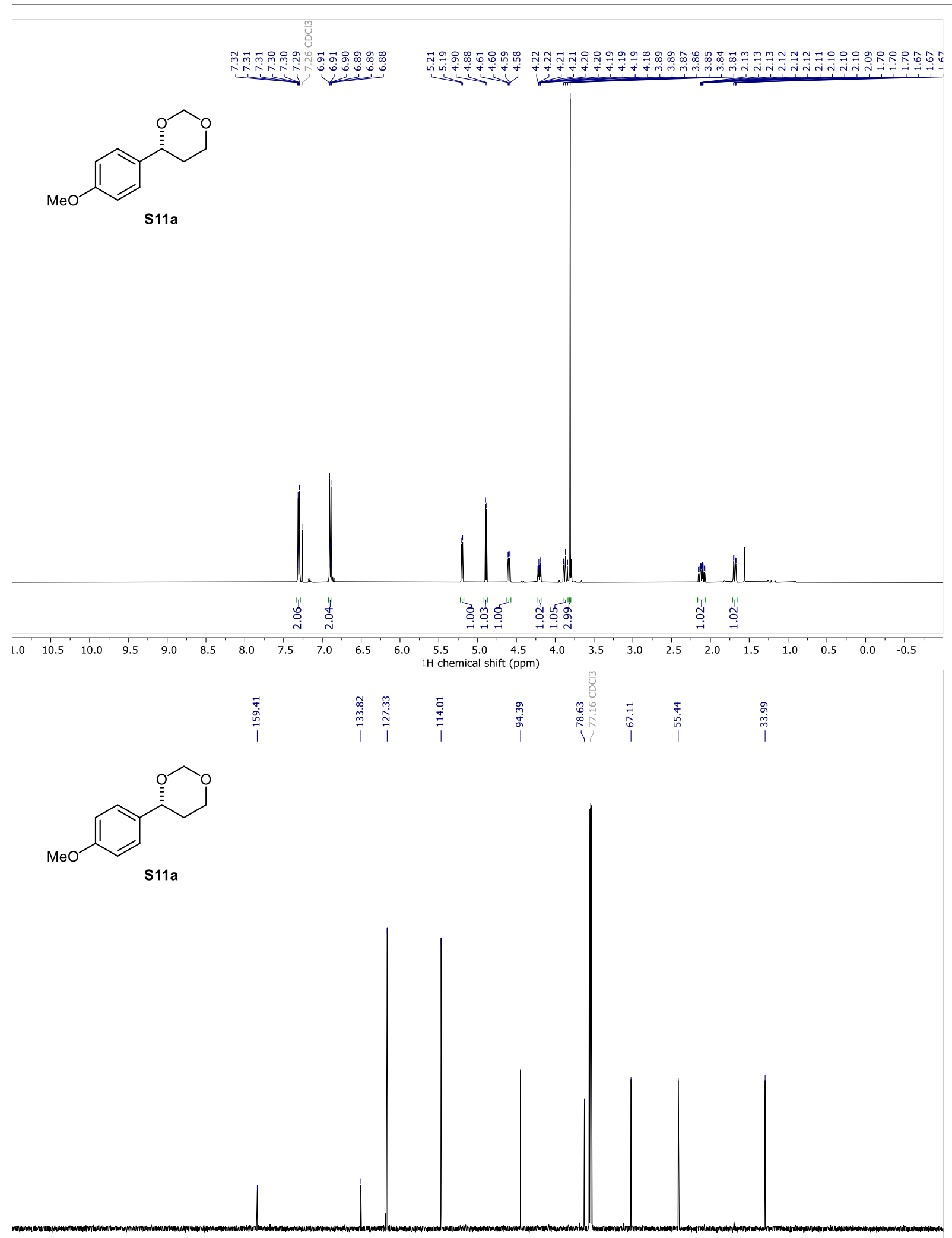

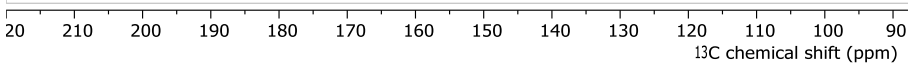

NMR spectra for compound S11a: ${ }^{1} \mathrm{H}(501 \mathrm{MHz})$ and ${ }^{13} \mathrm{C}(126 \mathrm{MHz})$, in $\mathrm{CDCl}_{3}$. 

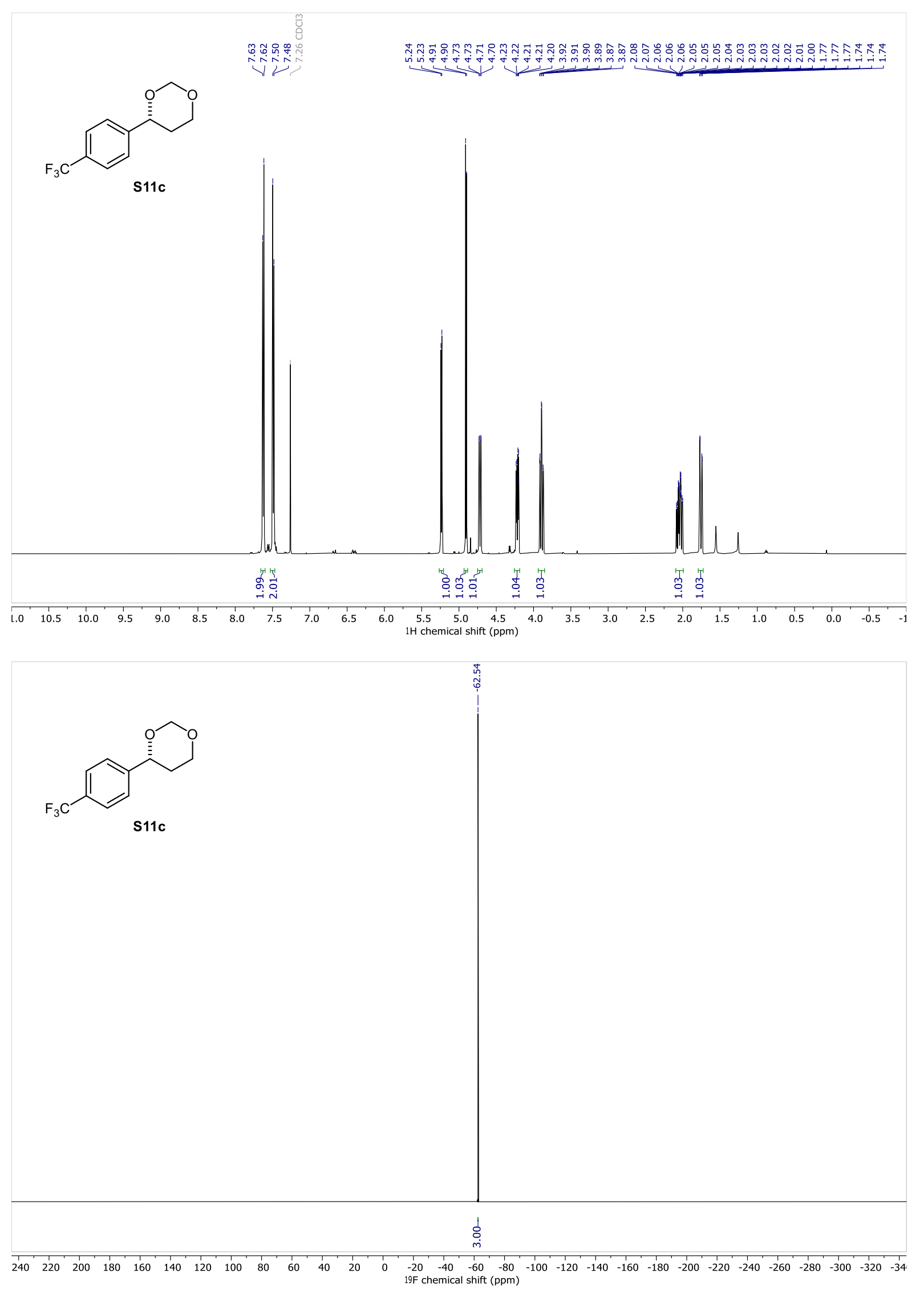

NMR spectra for compound S11c: ${ }^{1} \mathrm{H}(501 \mathrm{MHz})$ and ${ }^{19} \mathrm{~F}(471 \mathrm{MHz})$, in $\mathrm{CDCl}_{3}$. 

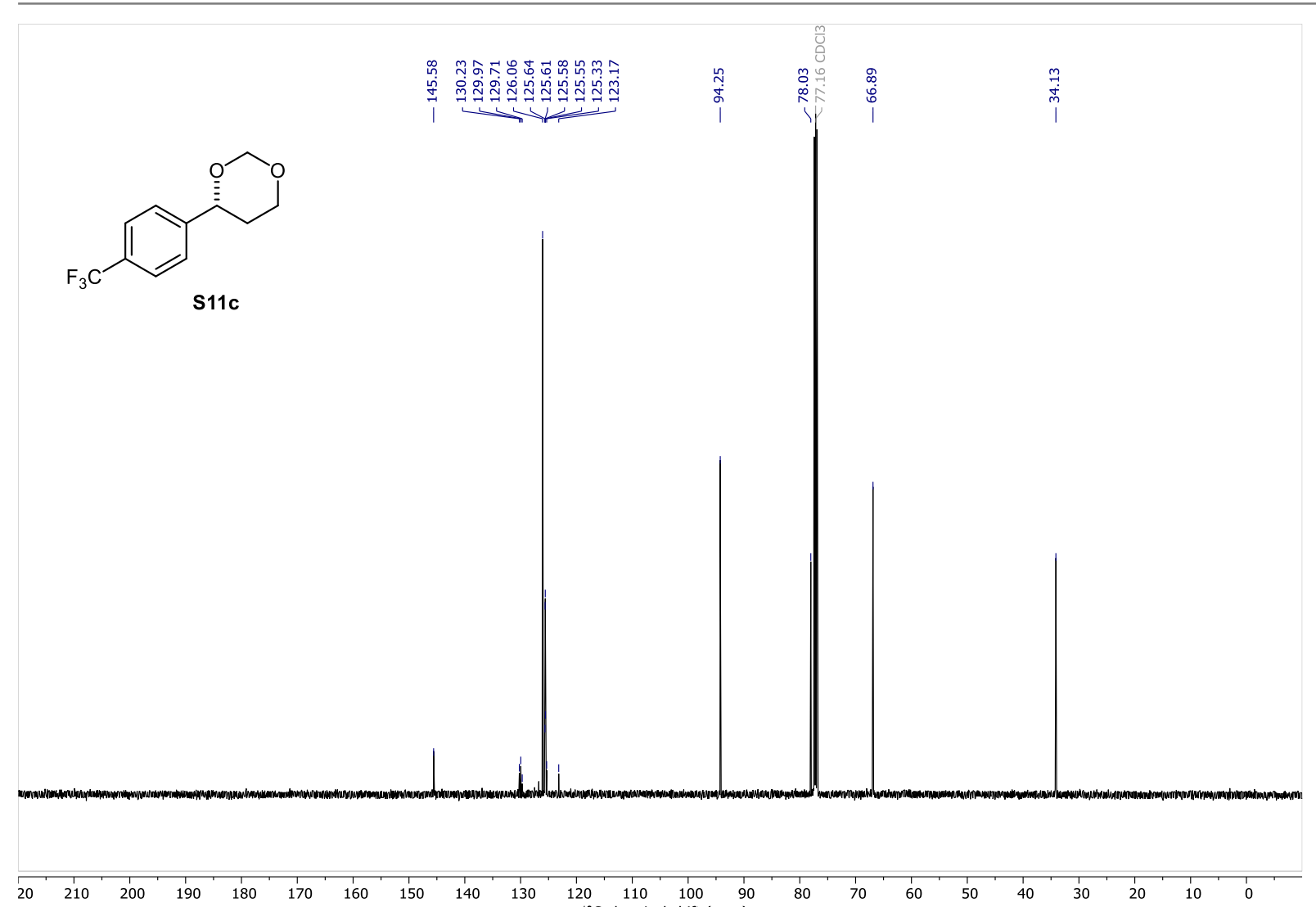

NMR spectra for compound S11c (continuation): ${ }^{13} \mathrm{C}(126 \mathrm{MHz})$, in $\mathrm{CDCl}_{3}$. 


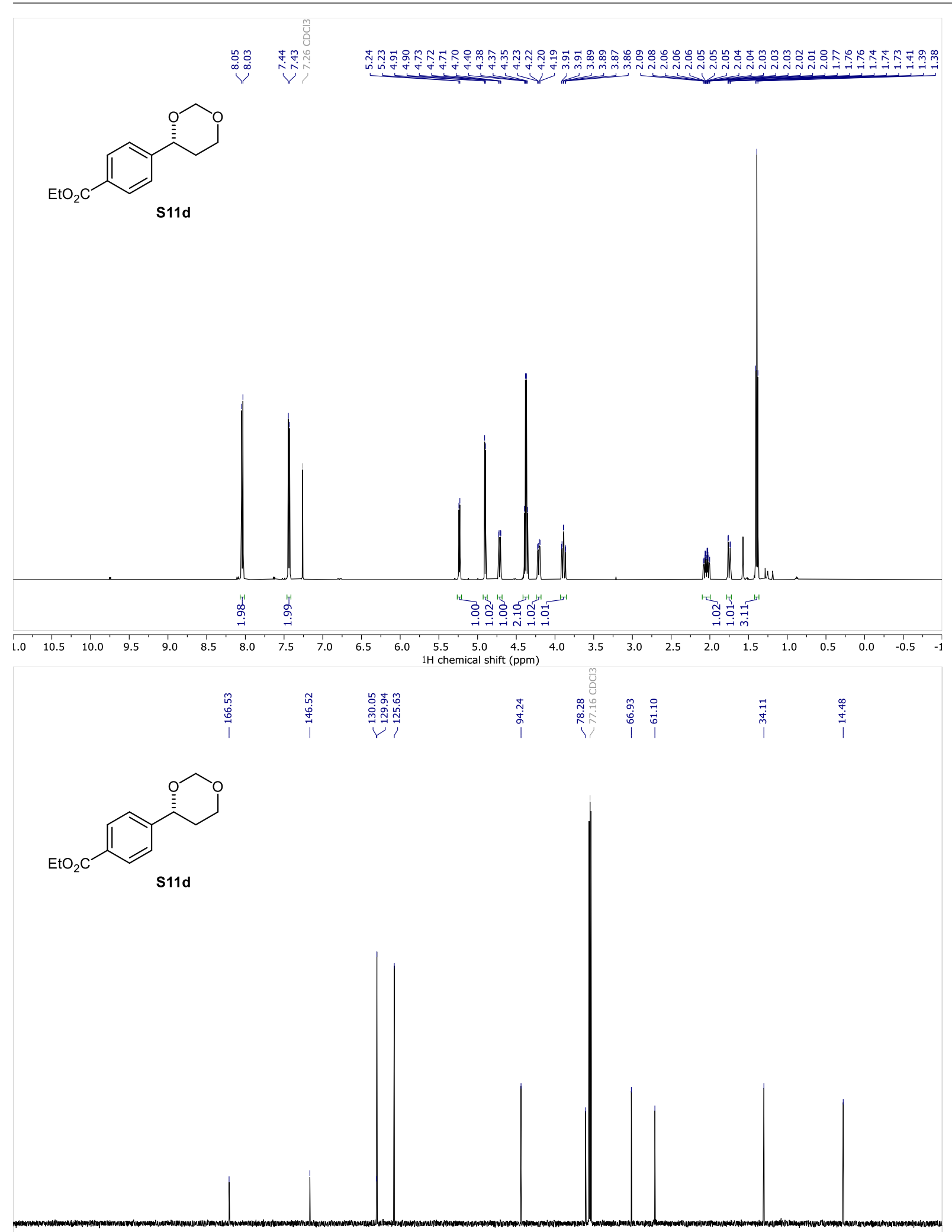

$\begin{array}{llllllllllll}210 & 200 & 190 & 180 & 170 & 160 & 150 & 140 & 130 & 120 & 110 & 100 \\ 13 \mathrm{C} \text { chemical shift (ppm) }\end{array}$

NMR spectra for compound S11d: ${ }^{1} \mathrm{H}(501 \mathrm{MHz})$ and ${ }^{13} \mathrm{C}(126 \mathrm{MHz})$, in $\mathrm{CDCl}_{3}$. 


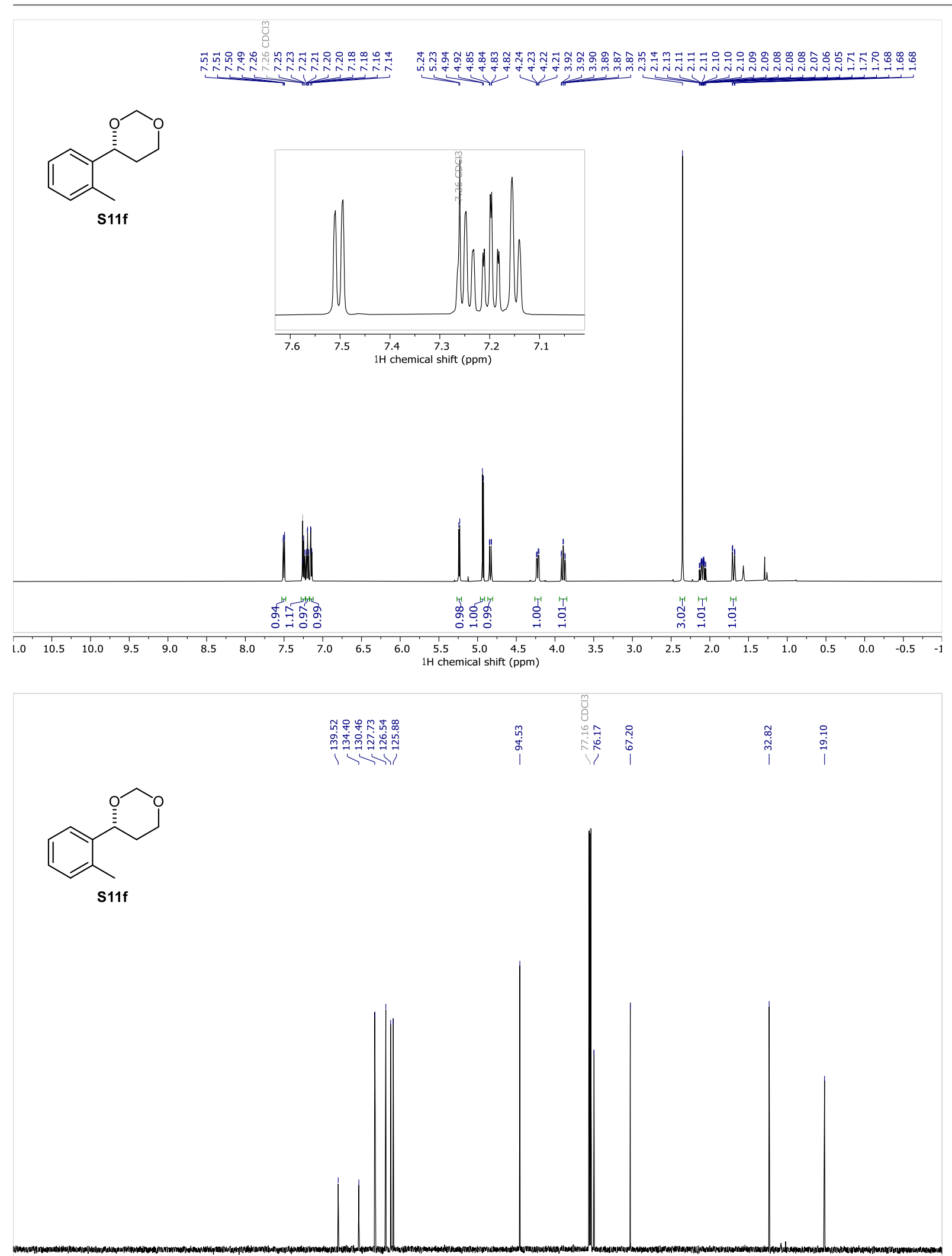

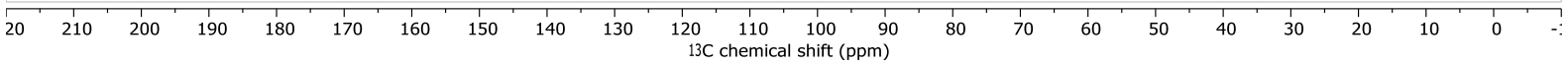

NMR spectra for compound S11f: ${ }^{1} \mathrm{H}(501 \mathrm{MHz})$ and ${ }^{13} \mathrm{C}(126 \mathrm{MHz})$, in $\mathrm{CDCl}_{3}$. 


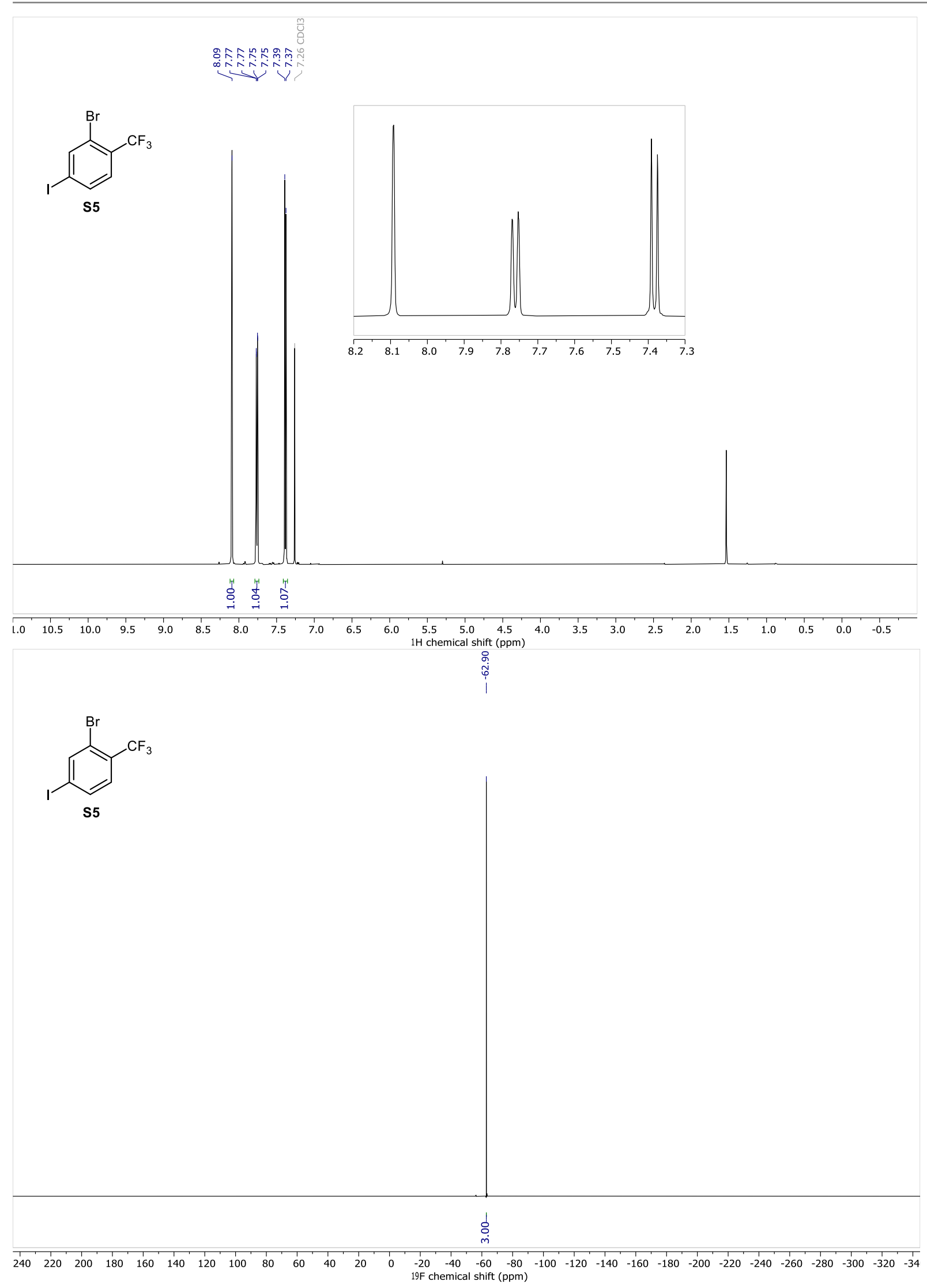

NMR spectra for compound S5: ${ }^{1} \mathrm{H}(501 \mathrm{MHz})$ and ${ }^{19} \mathrm{~F}(471 \mathrm{MHz})$, in $\mathrm{CDCl}_{3}$. 


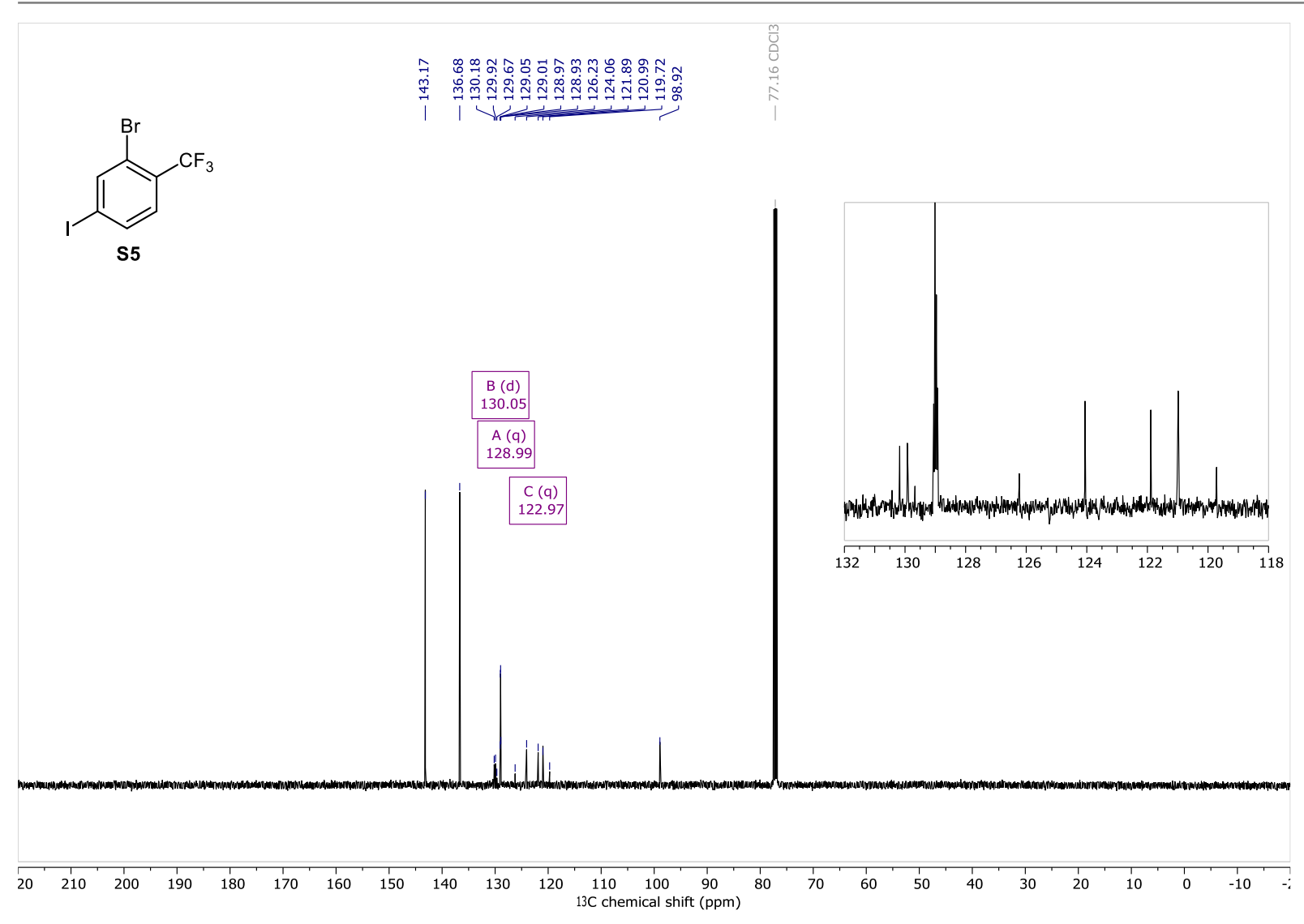

NMR spectra for compound S5 (continuation): ${ }^{13} \mathrm{C}(126 \mathrm{MHz})$, in $\mathrm{CDCl}_{3}$. 


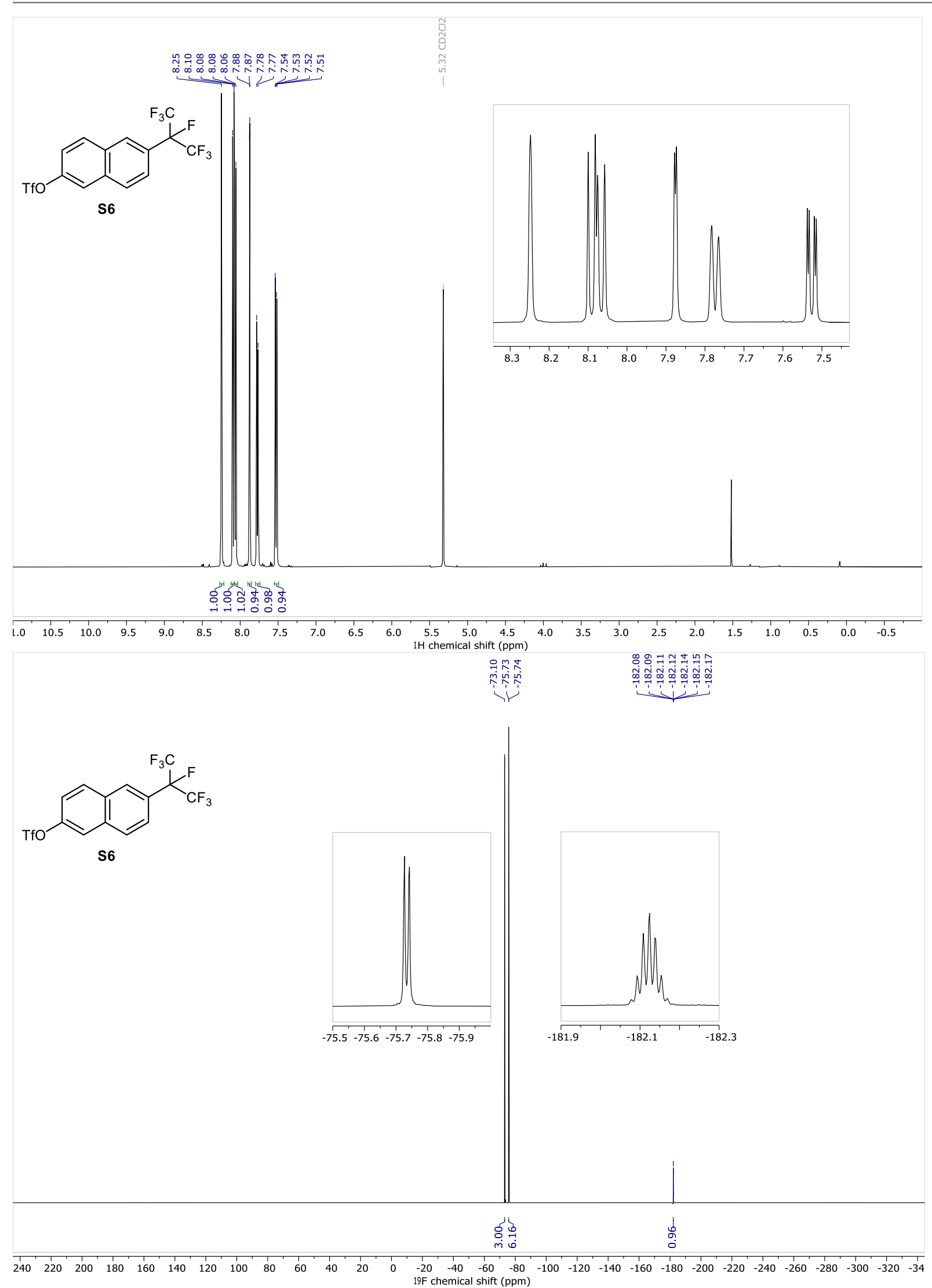

NMR spectra for compound S6: ${ }^{1} \mathrm{H}(501 \mathrm{MHz})$ and ${ }^{19} \mathrm{~F}(471 \mathrm{MHz})$, in $\mathrm{CD}_{2} \mathrm{Cl}_{2}$. 


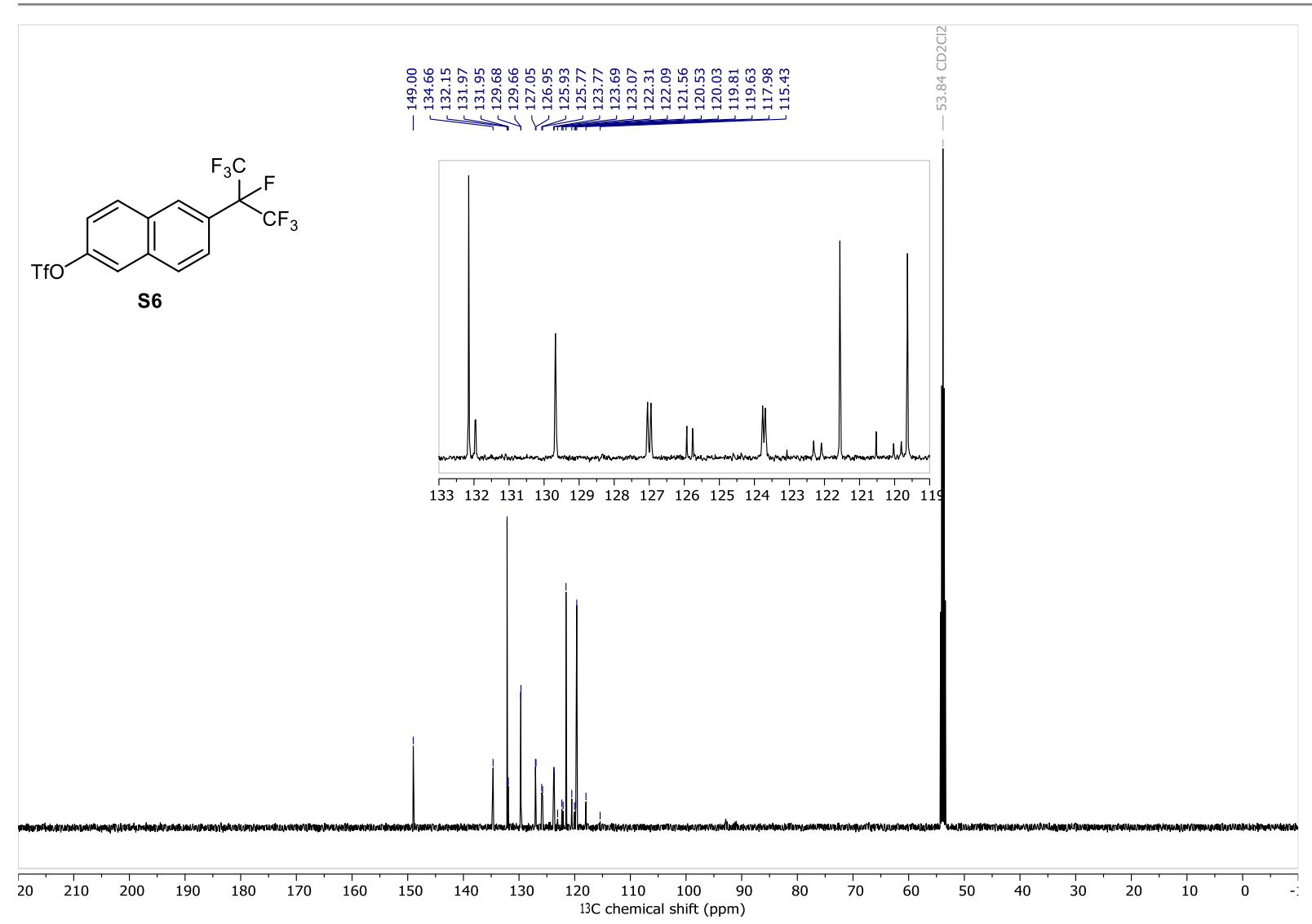

NMR spectra for compound $\mathbf{S 6}$ (continuation): ${ }^{13} \mathrm{C}(126 \mathrm{MHz})$, in $\mathrm{CD}_{2} \mathrm{Cl}_{2}$. 


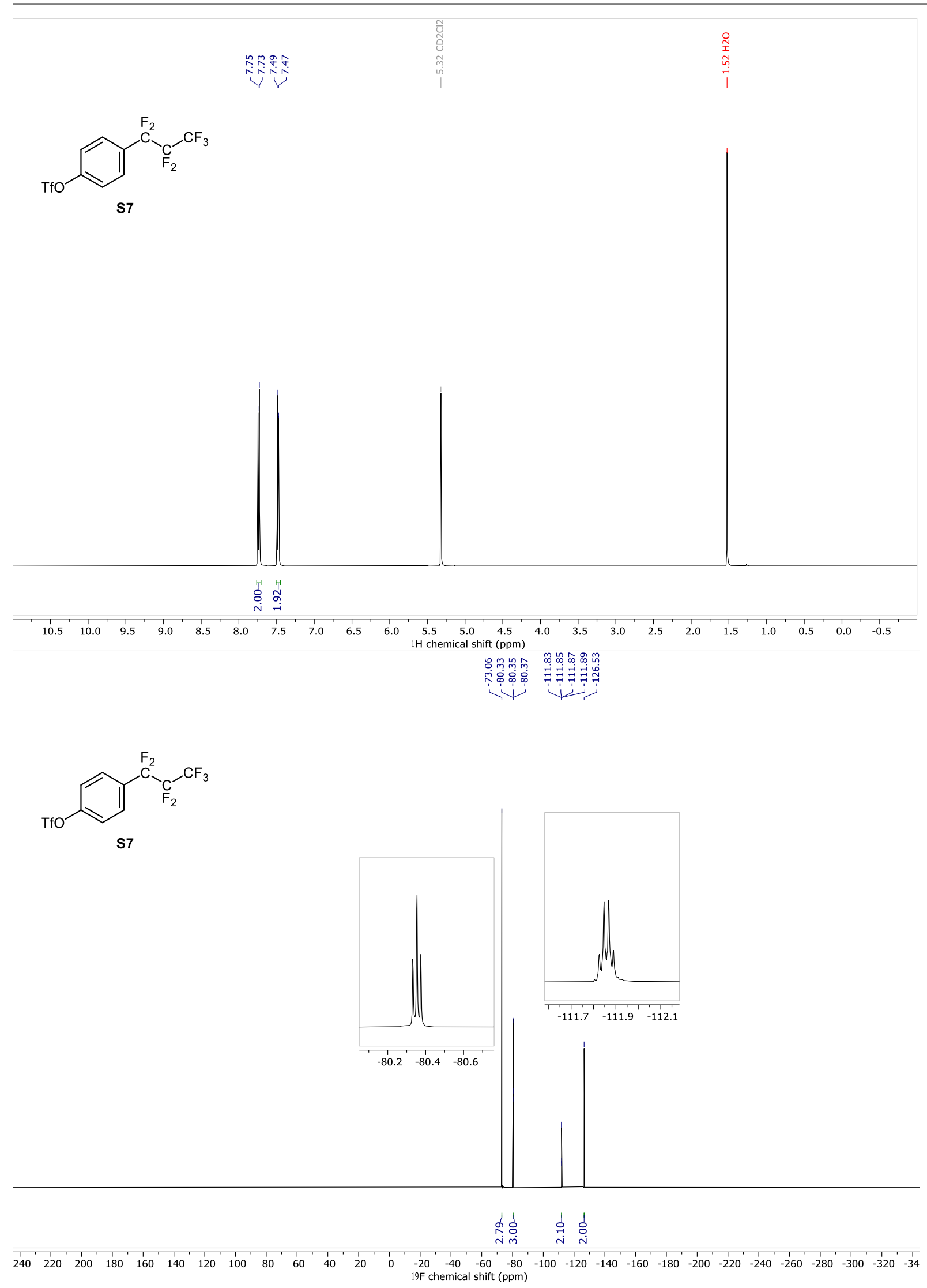

NMR spectra for compound S7: ${ }^{1} \mathrm{H}(501 \mathrm{MHz})$ and ${ }^{19} \mathrm{~F}(471 \mathrm{MHz})$, in $\mathrm{CD}_{2} \mathrm{Cl}_{2}$. 


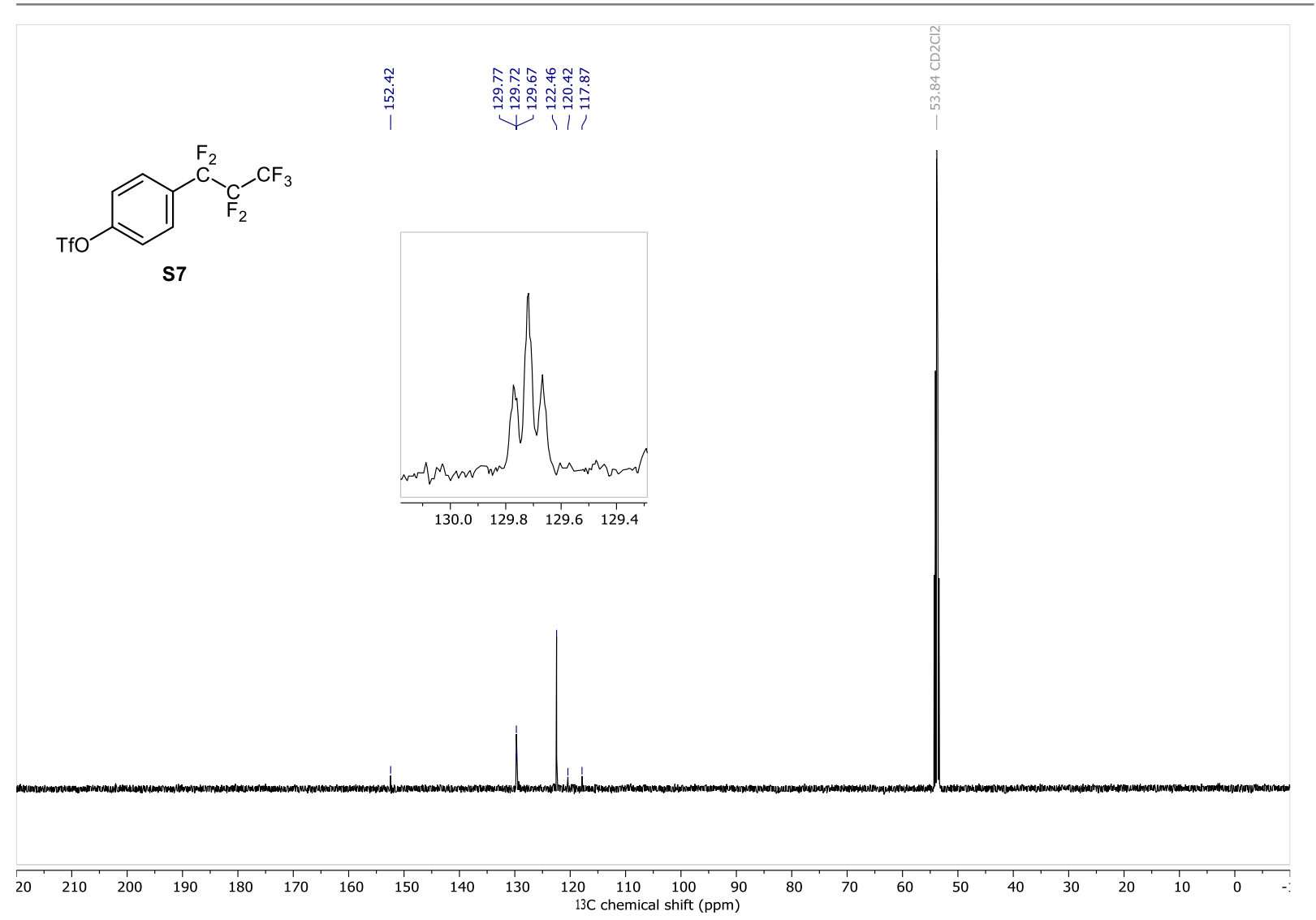

NMR spectra for compound $\mathbf{S 7}$ (continuation): ${ }^{13} \mathrm{C}\left(126 \mathrm{MHz}\right.$ ), in $\mathrm{CD}_{2} \mathrm{Cl}_{2}$. 


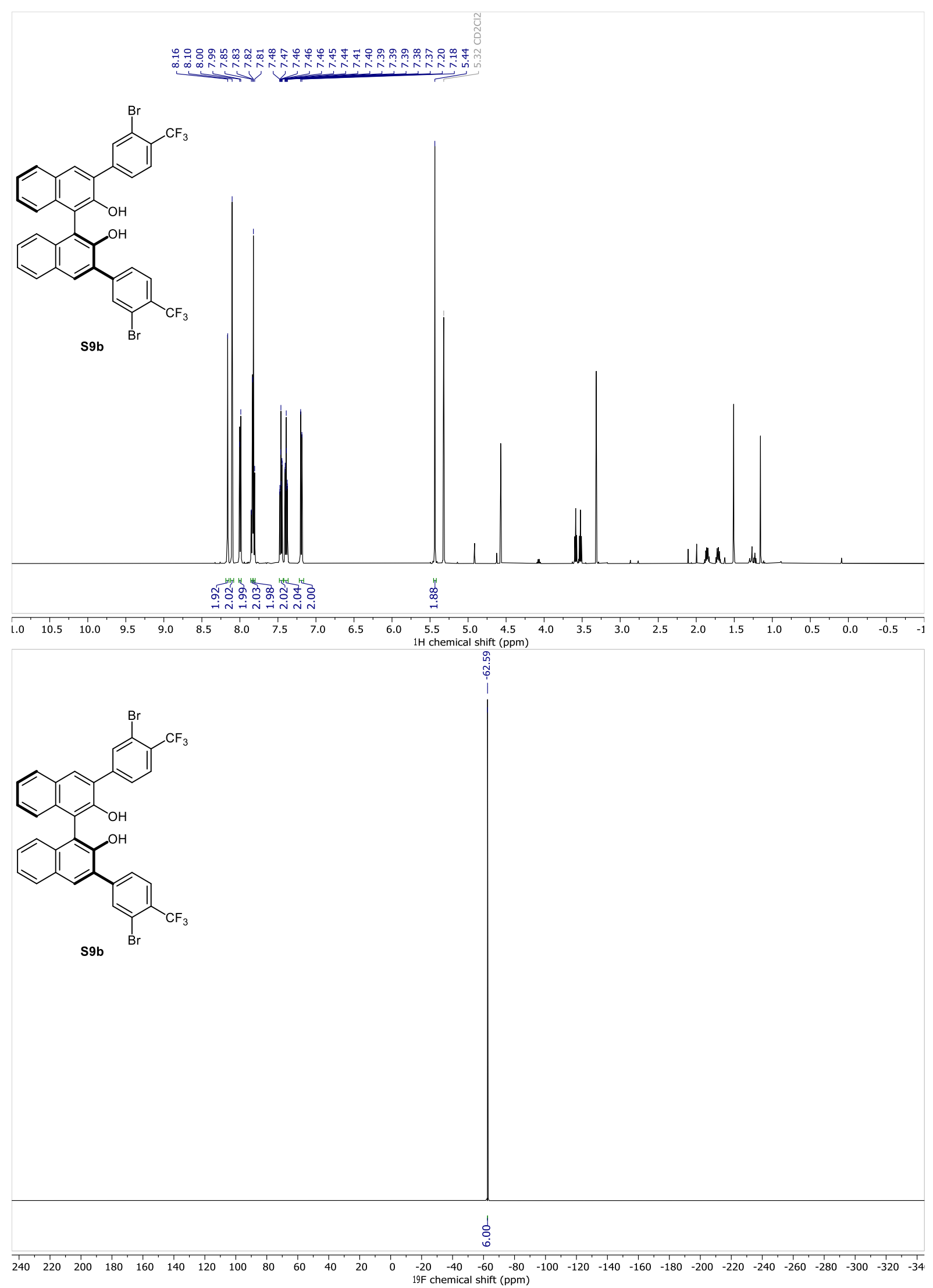

NMR spectra for compound S9b: ${ }^{1} \mathrm{H}(501 \mathrm{MHz})$ and ${ }^{19} \mathrm{~F}(471 \mathrm{MHz})$, in $\mathrm{CD}_{2} \mathrm{Cl}_{2}$. 


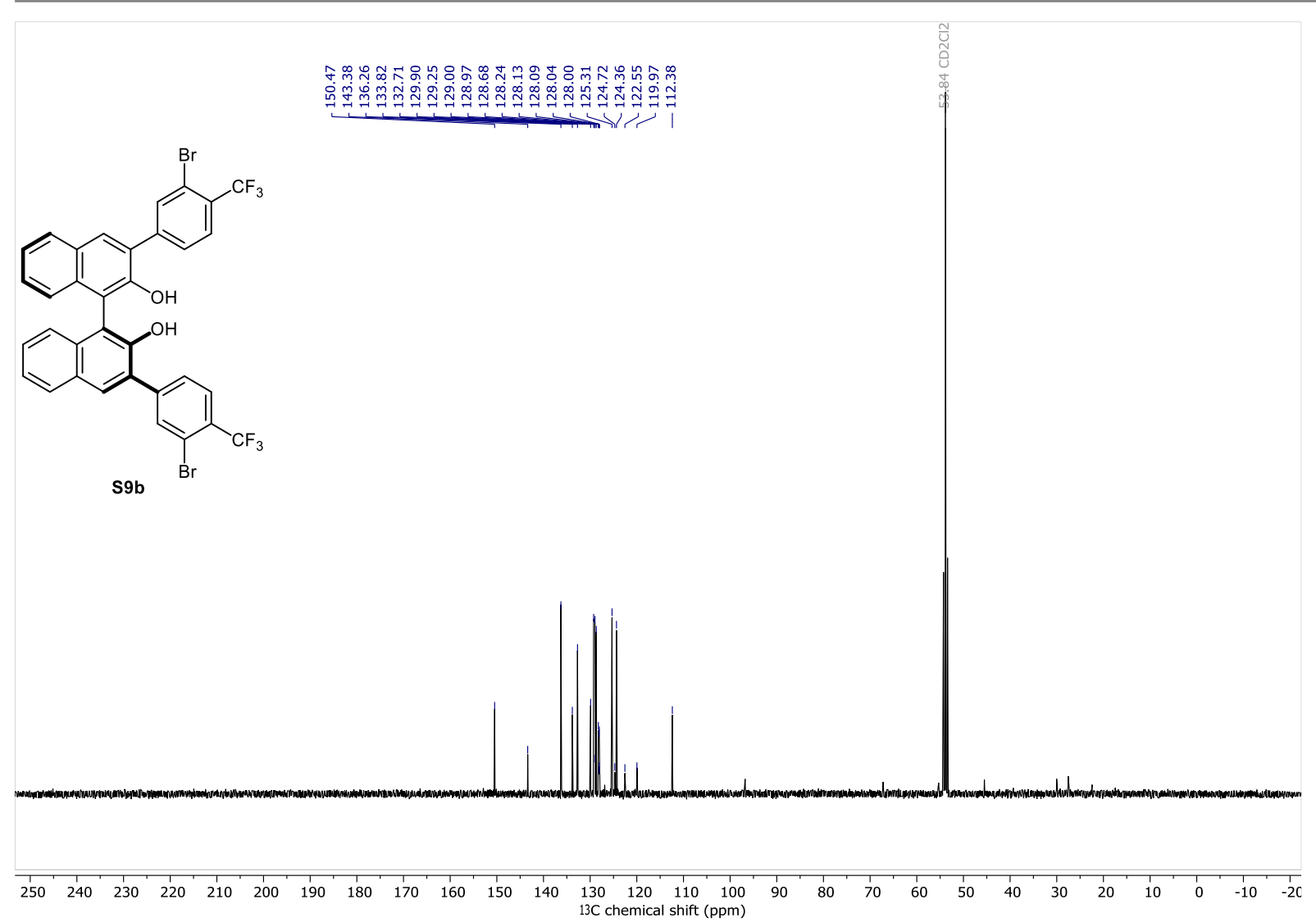

NMR spectra for compound S9b (continuation): ${ }^{13} \mathrm{C}(126 \mathrm{MHz})$, in $\mathrm{CD}_{2} \mathrm{Cl}_{2}$. 


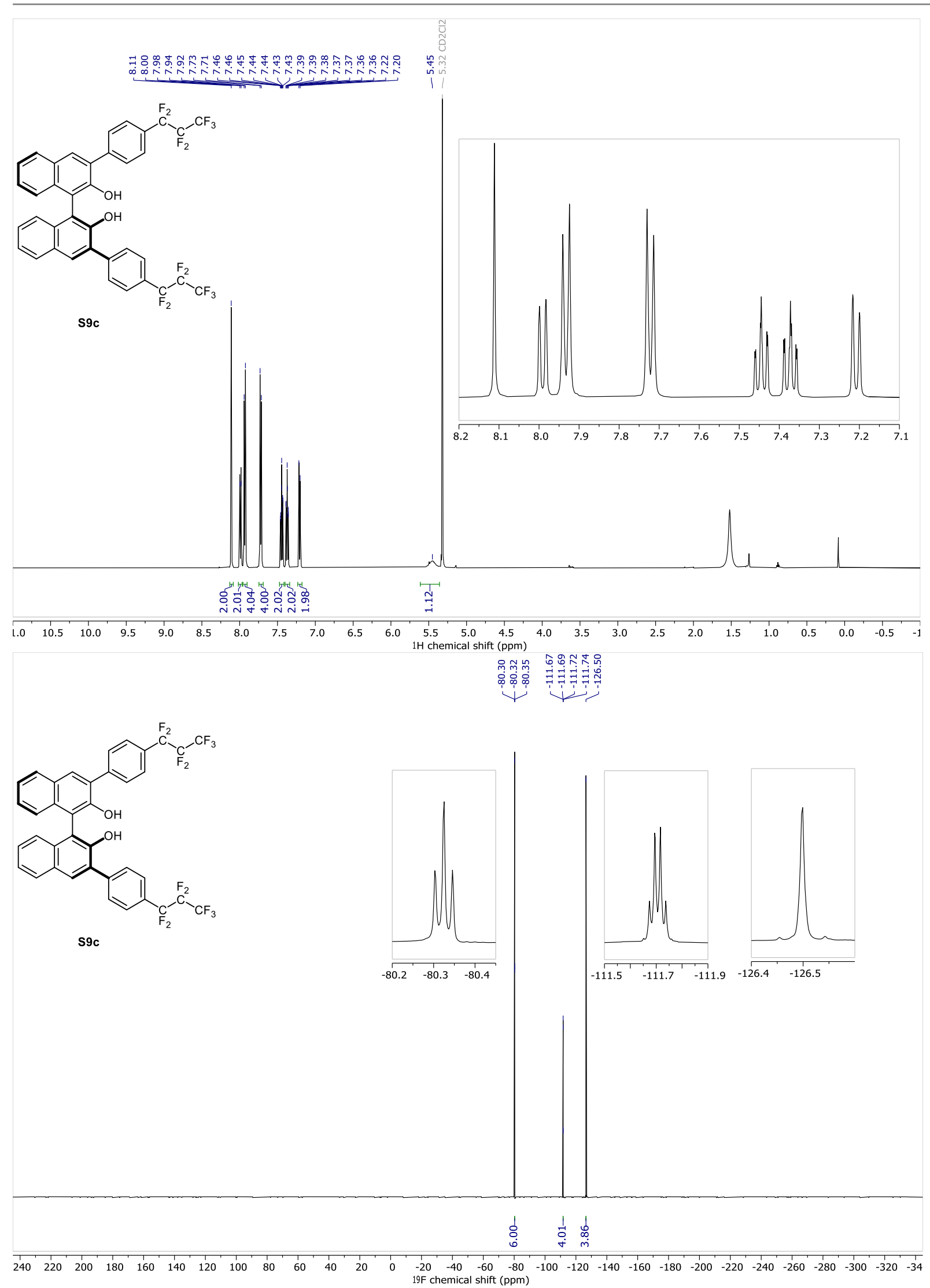

NMR spectra for compound S9c: ${ }^{1} \mathrm{H}(501 \mathrm{MHz})$ and ${ }^{19} \mathrm{~F}(471 \mathrm{MHz})$, in $\mathrm{CD}_{2} \mathrm{Cl}_{2}$. 


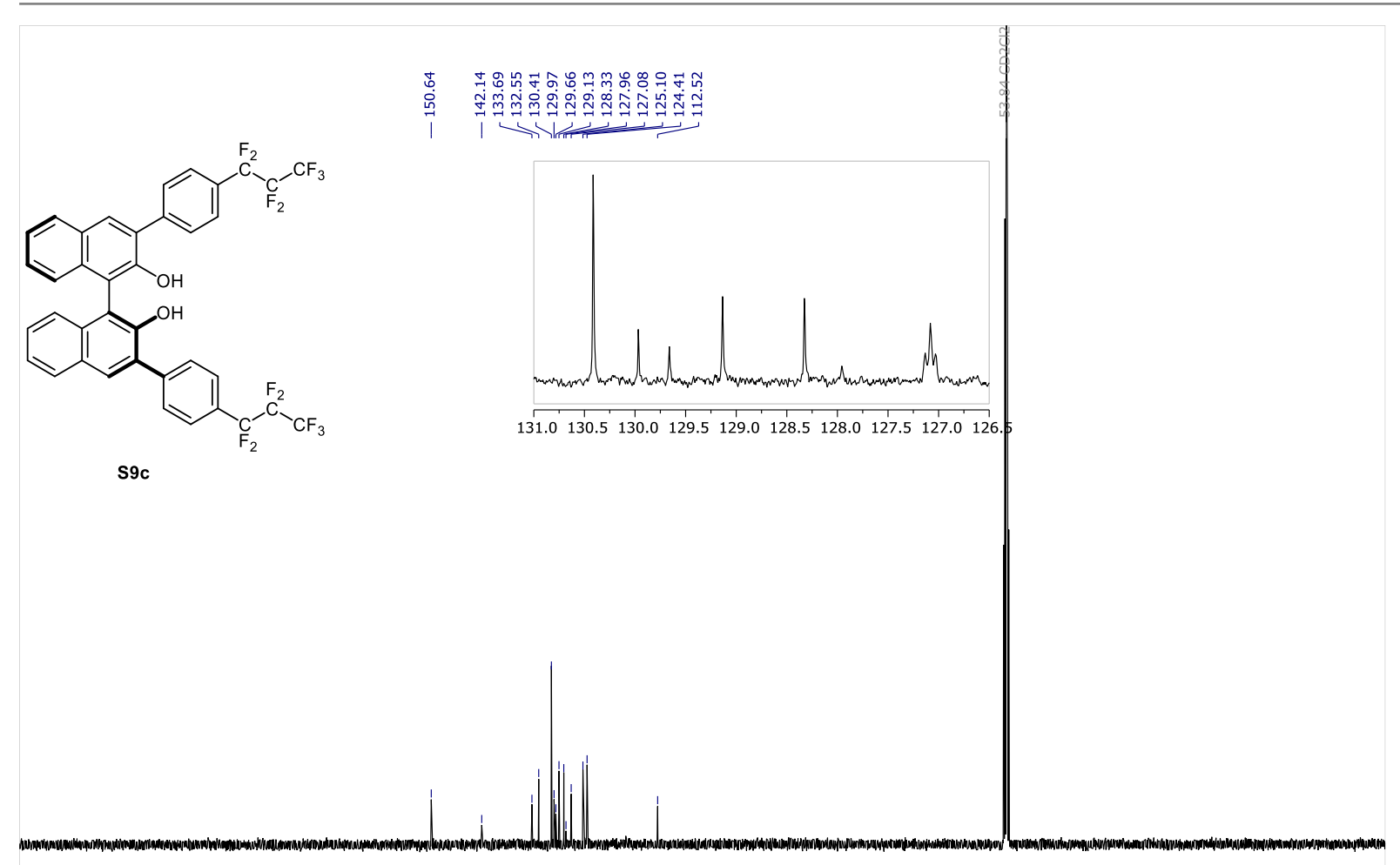

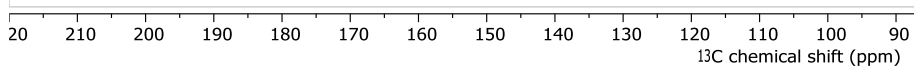

NMR spectra for compound S9c (continuation): ${ }^{13} \mathrm{C}(126 \mathrm{MHz})$, in $\mathrm{CD}_{2} \mathrm{Cl}_{2}$. 


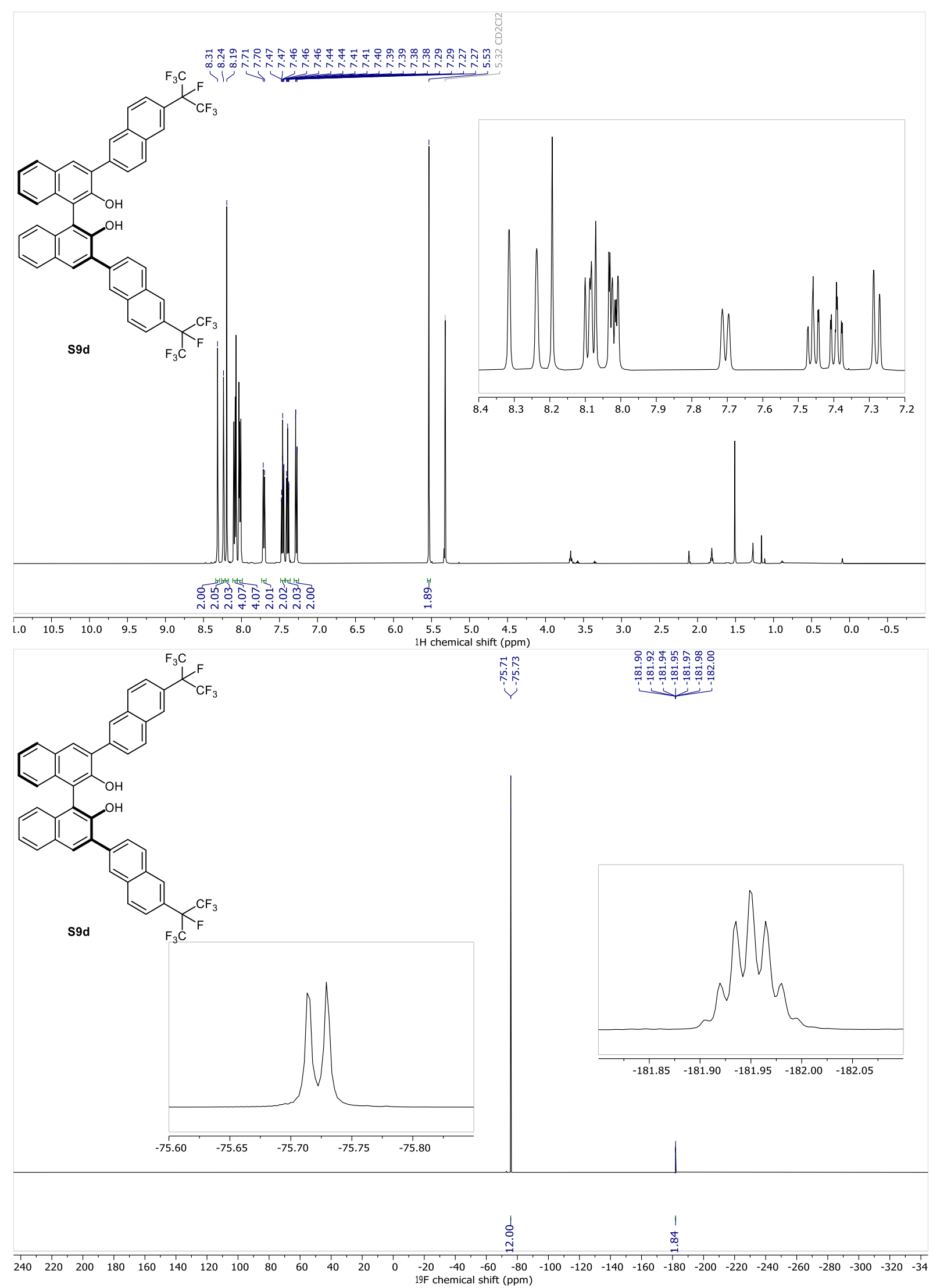

NMR spectra for compound S9d: ${ }^{1} \mathrm{H}(501 \mathrm{MHz})$ and ${ }^{19} \mathrm{~F}(471 \mathrm{MHz})$, in $\mathrm{CD}_{2} \mathrm{Cl}_{2}$. 

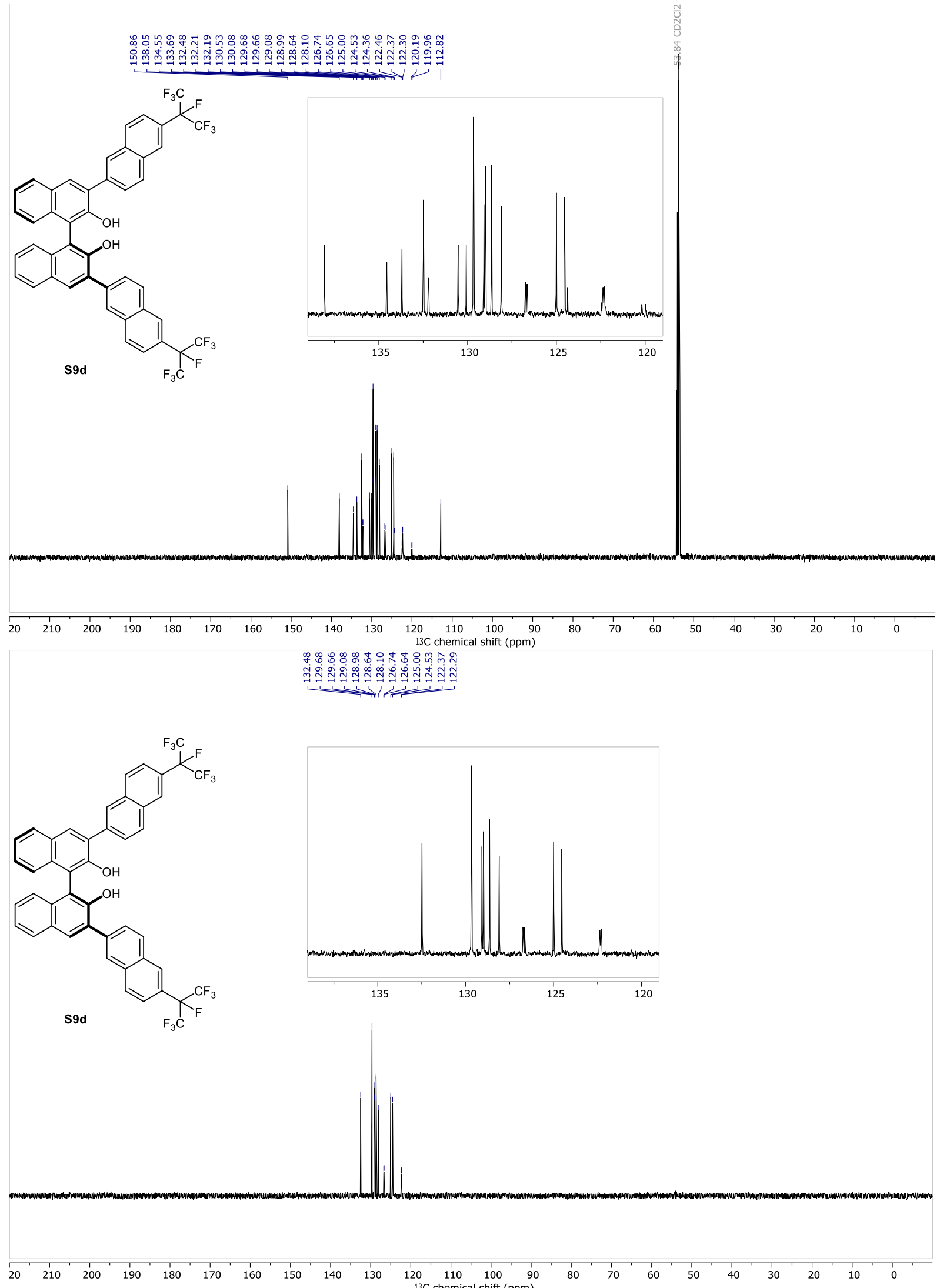

NMR spectra for compound S9d (continuation): ${ }^{13} \mathrm{C}(126 \mathrm{MHz})$ and DEPT-135 (126 MHz), in $\mathrm{CD}_{2} \mathrm{Cl}_{2}$. 


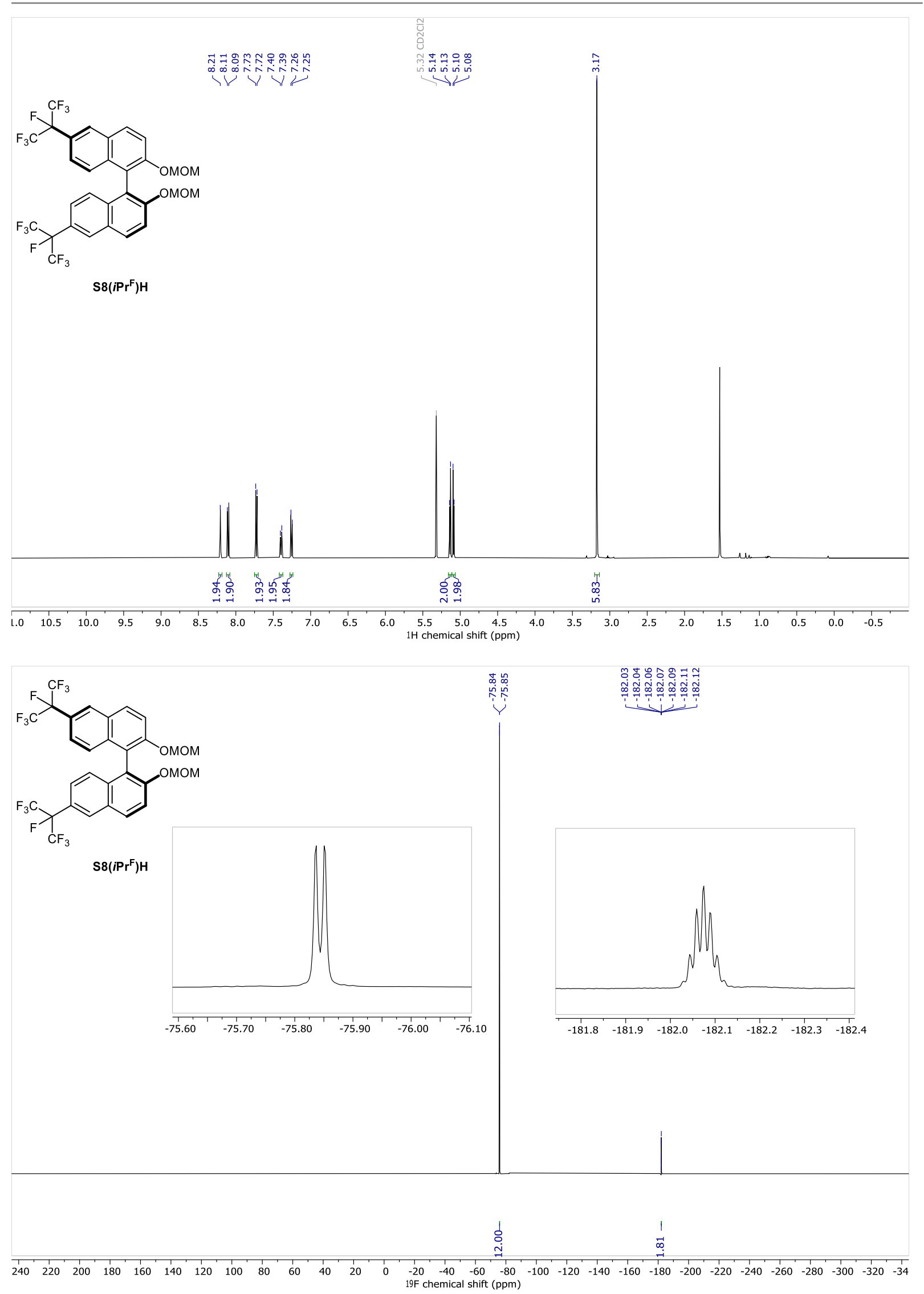

NMR spectra for compound S8(iPrF) H: ${ }^{1} \mathrm{H}(501 \mathrm{MHz})$ and ${ }^{19} \mathrm{~F}(471 \mathrm{MHz})$, in $\mathrm{CD}_{2} \mathrm{Cl}_{2}$. 


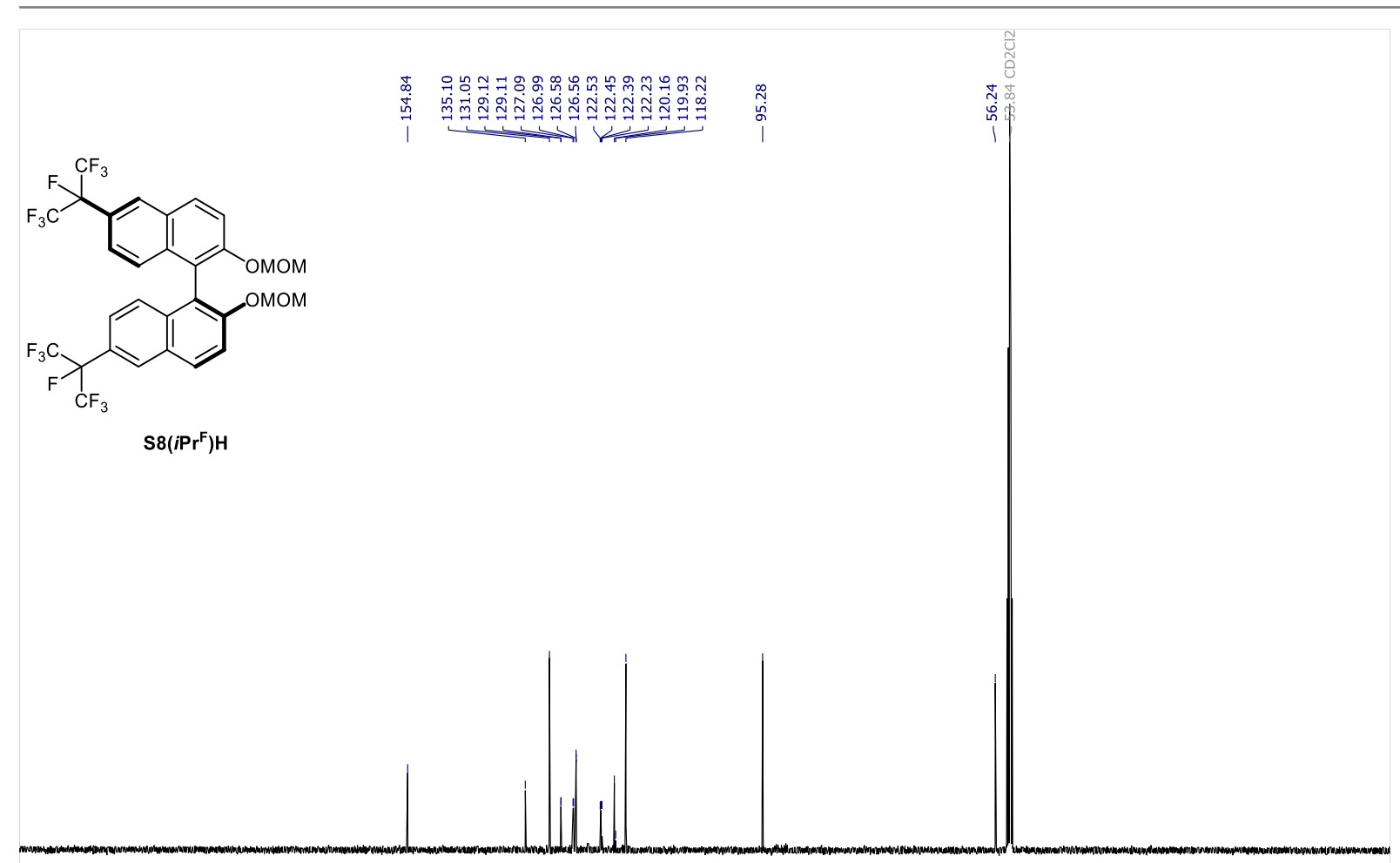

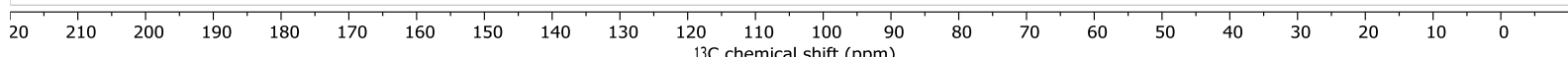

NMR spectra for compound $\mathbf{S 8}(\mathbf{P P r}) \mathbf{H}$ (continuation): ${ }^{13} \mathrm{C}(126 \mathrm{MHz})$, in $\mathrm{CD}_{2} \mathrm{Cl}_{2}$. 


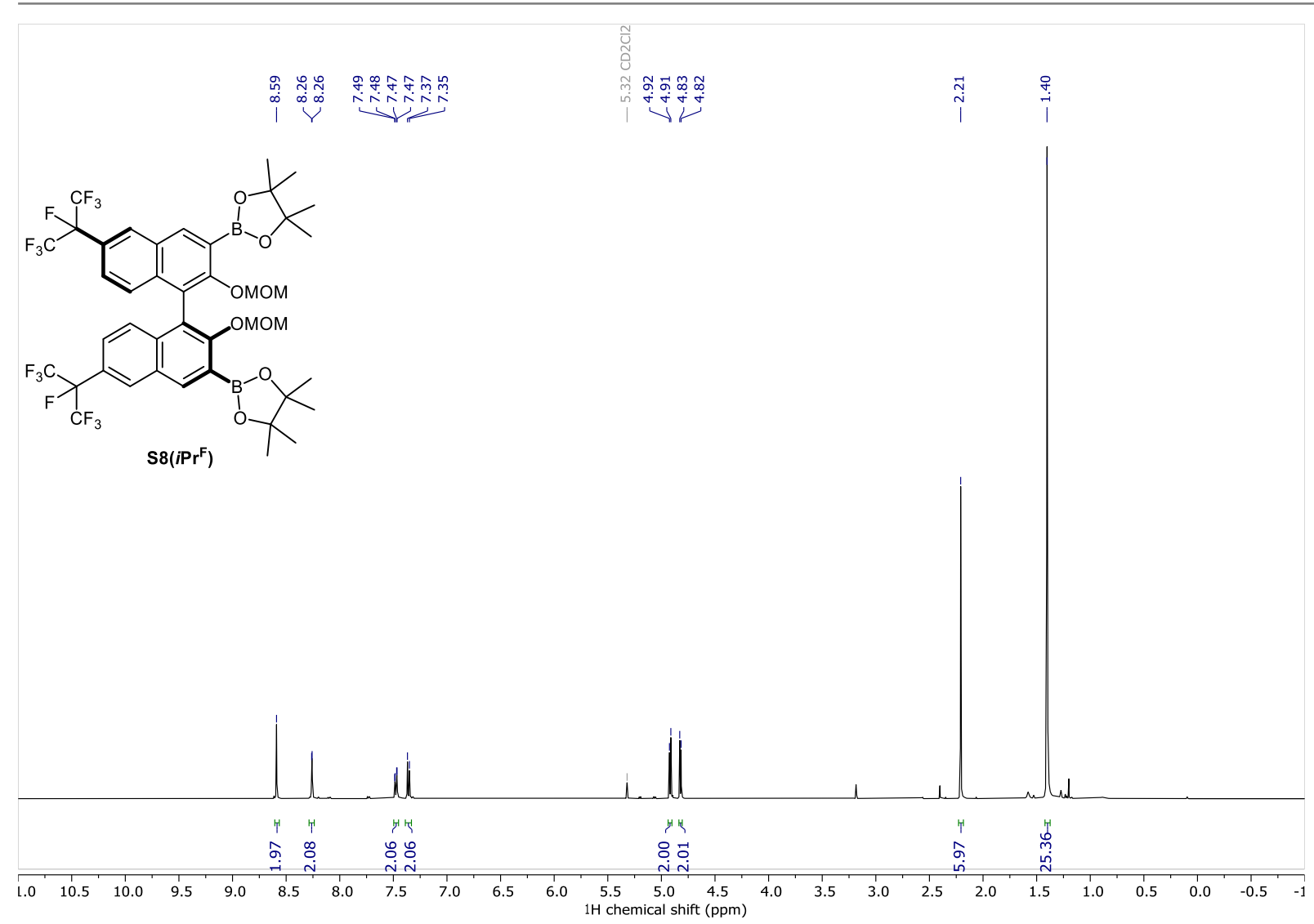<smiles>COc1c(B2OC(C)(C)C(C)(C)O2)cc2cc(C(F)(F)C(F)(F)F)ccc2c1-c1c(O)c(B2OC(C)(C)C(C)(C)O2)cc2cc(C(F)(F)F)ccc12</smiles>
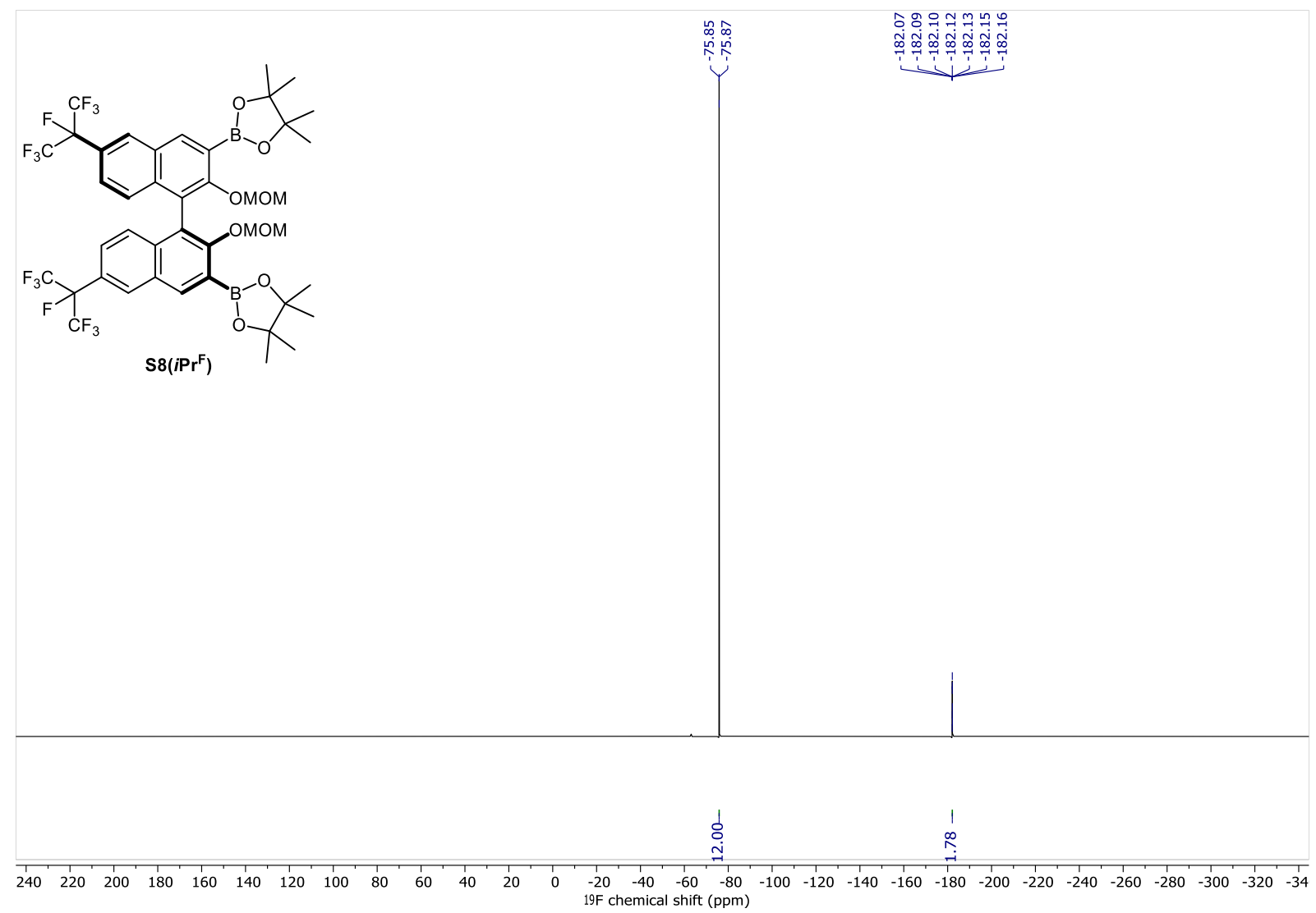

NMR spectra for compound S8(iPrF): ${ }^{1} \mathrm{H}(501 \mathrm{MHz})$ and ${ }^{19} \mathrm{~F}(471 \mathrm{MHz})$, in $\mathrm{CD}_{2} \mathrm{Cl}_{2}$. 


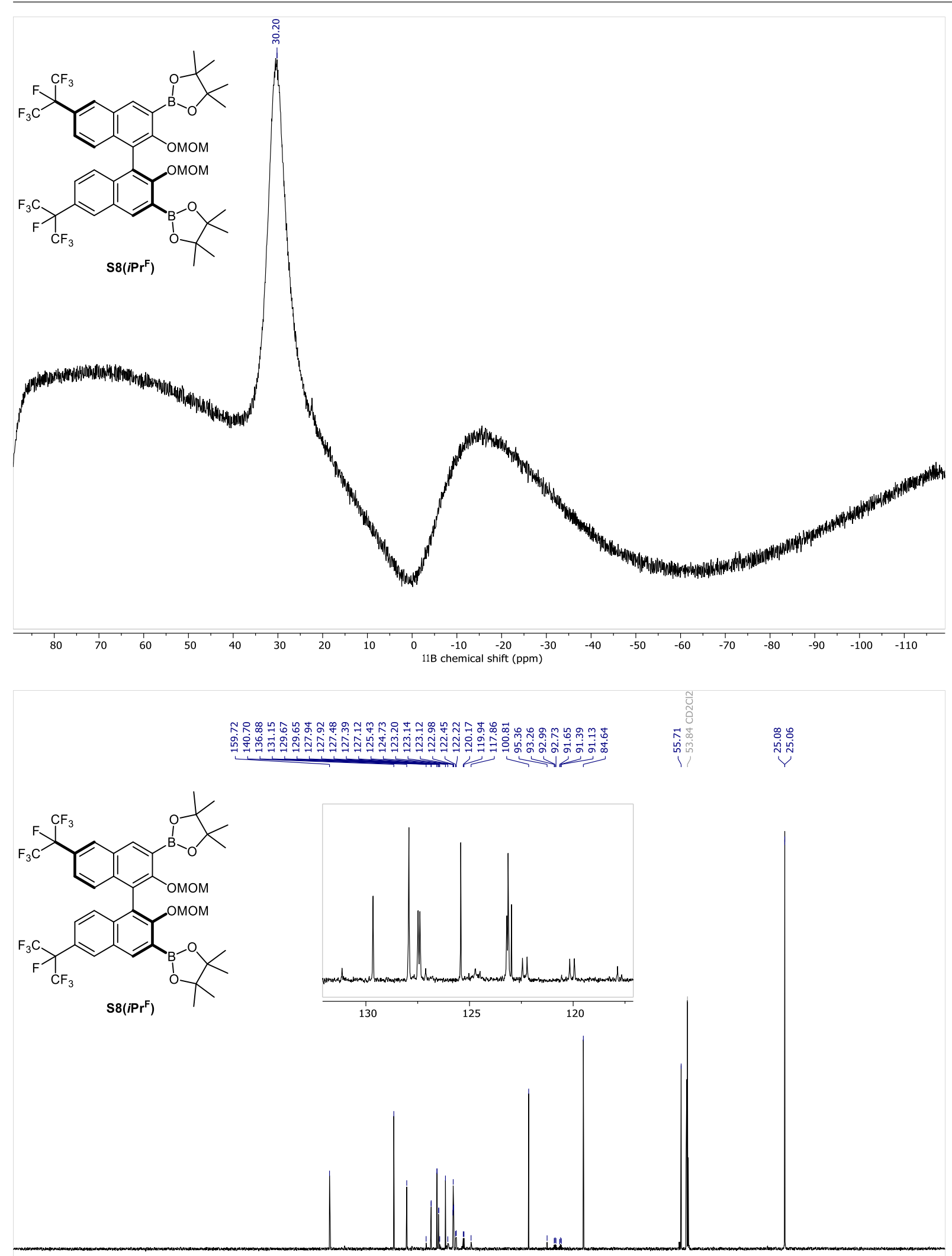

\begin{tabular}{lllllllllllllllllllllllllllllllll}
\hline & 150 & 240 & 230 & 220 & 210 & 200 & 190 & 180 & 170 & 160 & 150 & 140 & 130 & 120 & 110 & 100 & 90 & 80 & 70 & 60 & 50 & 40 & 30 & 20 & 10 & 0 & -10 & $-2 \mathrm{C}$
\end{tabular}

NMR spectra for compound S8( $\left(\mathrm{Pr}^{\mathrm{F}}\right)$ (continuation): ${ }^{11} \mathrm{~B}(161 \mathrm{MHz})$ and ${ }^{13} \mathrm{C}(126 \mathrm{MHz})$, in $\mathrm{CD}_{2} \mathrm{Cl}_{2}$. 


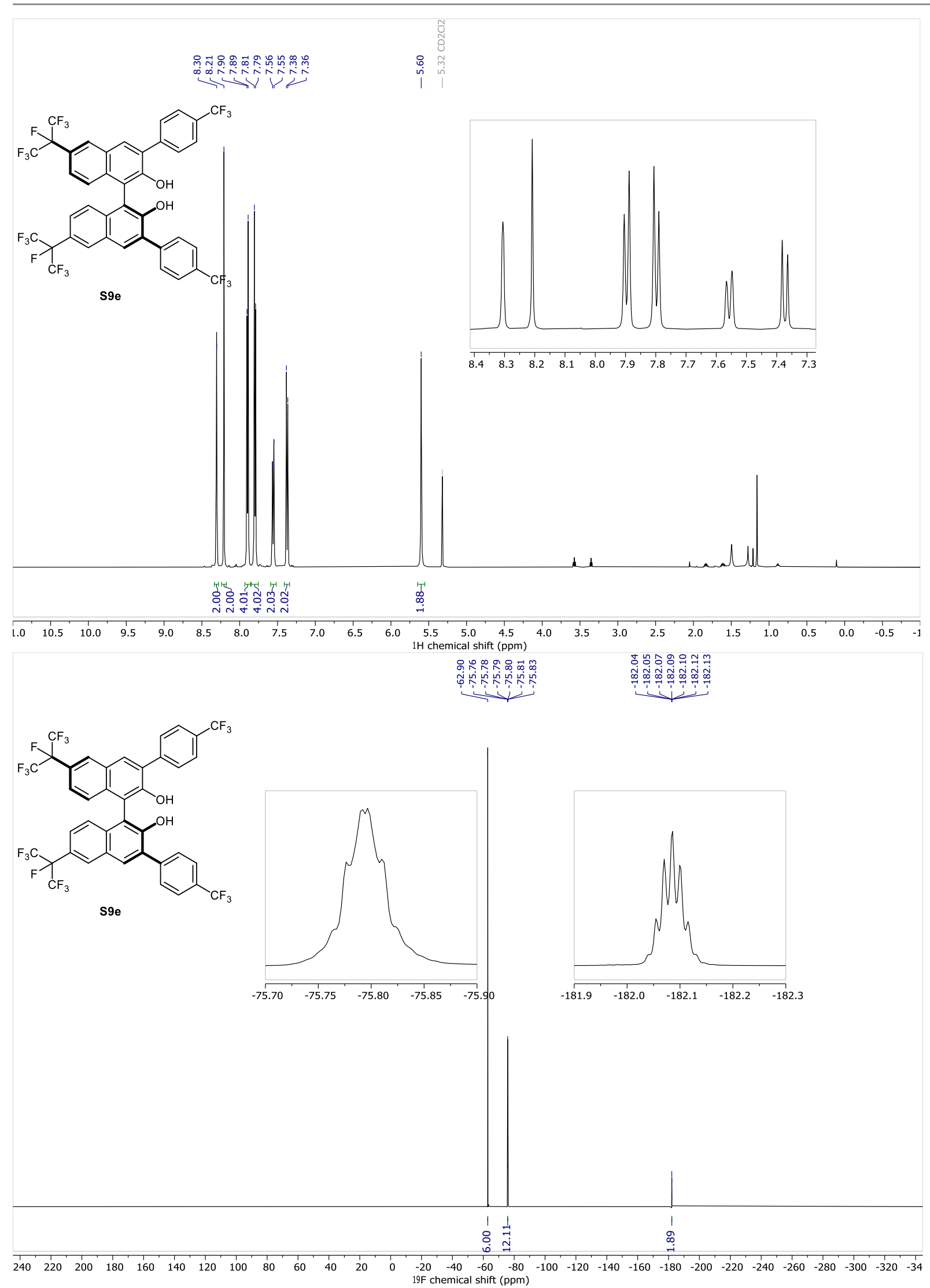

NMR spectra for compound S9e: ${ }^{1} \mathrm{H}(501 \mathrm{MHz})$ and ${ }^{19} \mathrm{~F}(471 \mathrm{MHz})$, in $\mathrm{CD}_{2} \mathrm{Cl}_{2}$. 
<smiles>Oc1c(-c2ccc(C(F)(F)F)cc2)cc2cc(C(F)(F)F)ccc2c1-c1c(O)c(-c2ccc(C(F)(F)F)cc2)cc2cc(C(F)(F)C(F)(F)F)ccc12</smiles>

S9e

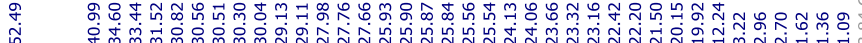

। व 90
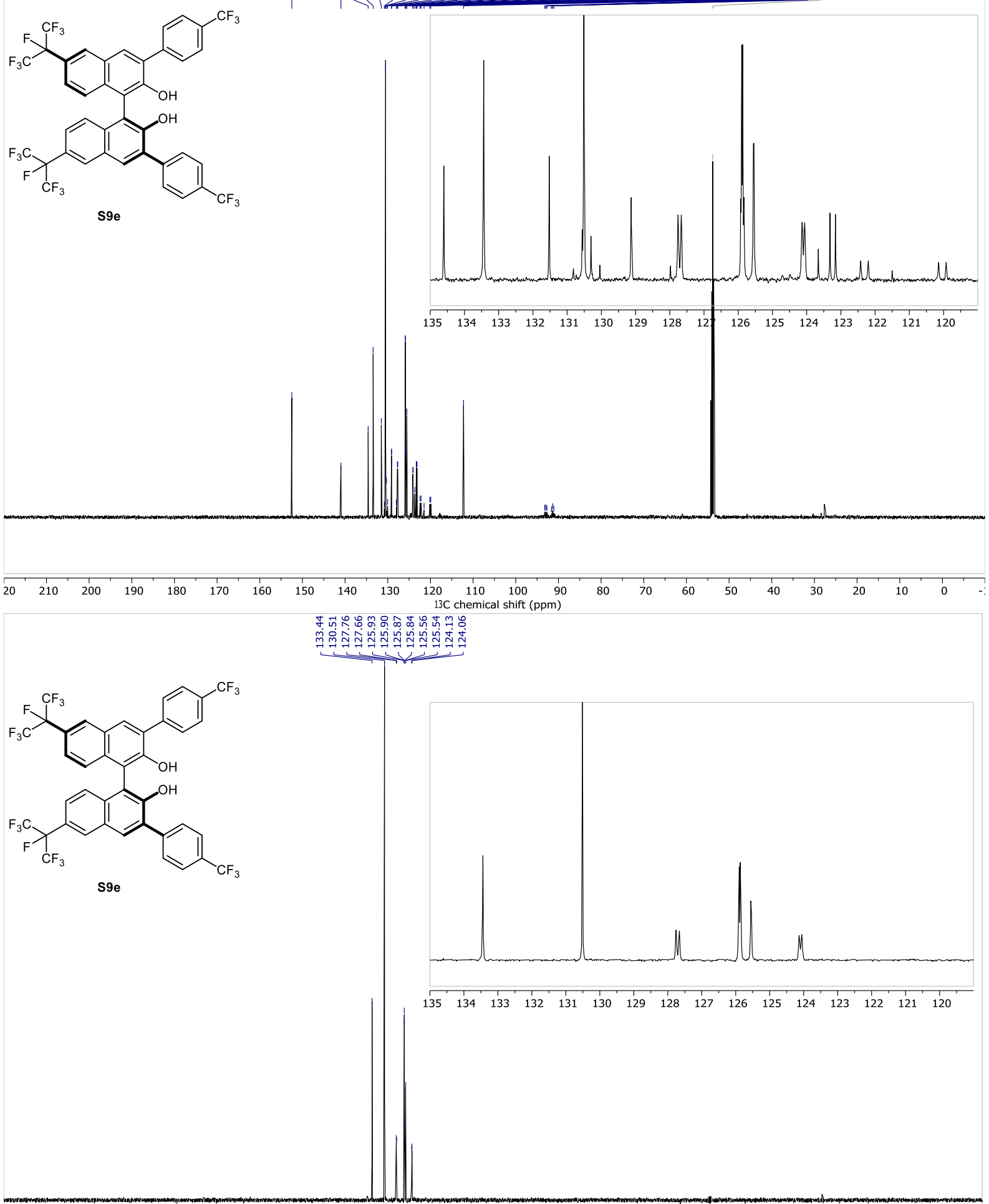

$\begin{array}{lllllllllllllllllllllllllll}1 & 210 & 200 & 190 & 180 & 170 & 160 & 150 & 140 & 130 & 120 & 110 & 100 & 90 & 80 & 70 & 60 & 50 & 40 & 30 & 20 & 10 & 0 & 1\end{array}$

NMR spectra for compound S9e (continuation): ${ }^{13} \mathrm{C}(126 \mathrm{MHz})$ and DEPT-135 (126 MHz), in $\mathrm{CD}_{2} \mathrm{Cl}_{2}$. 

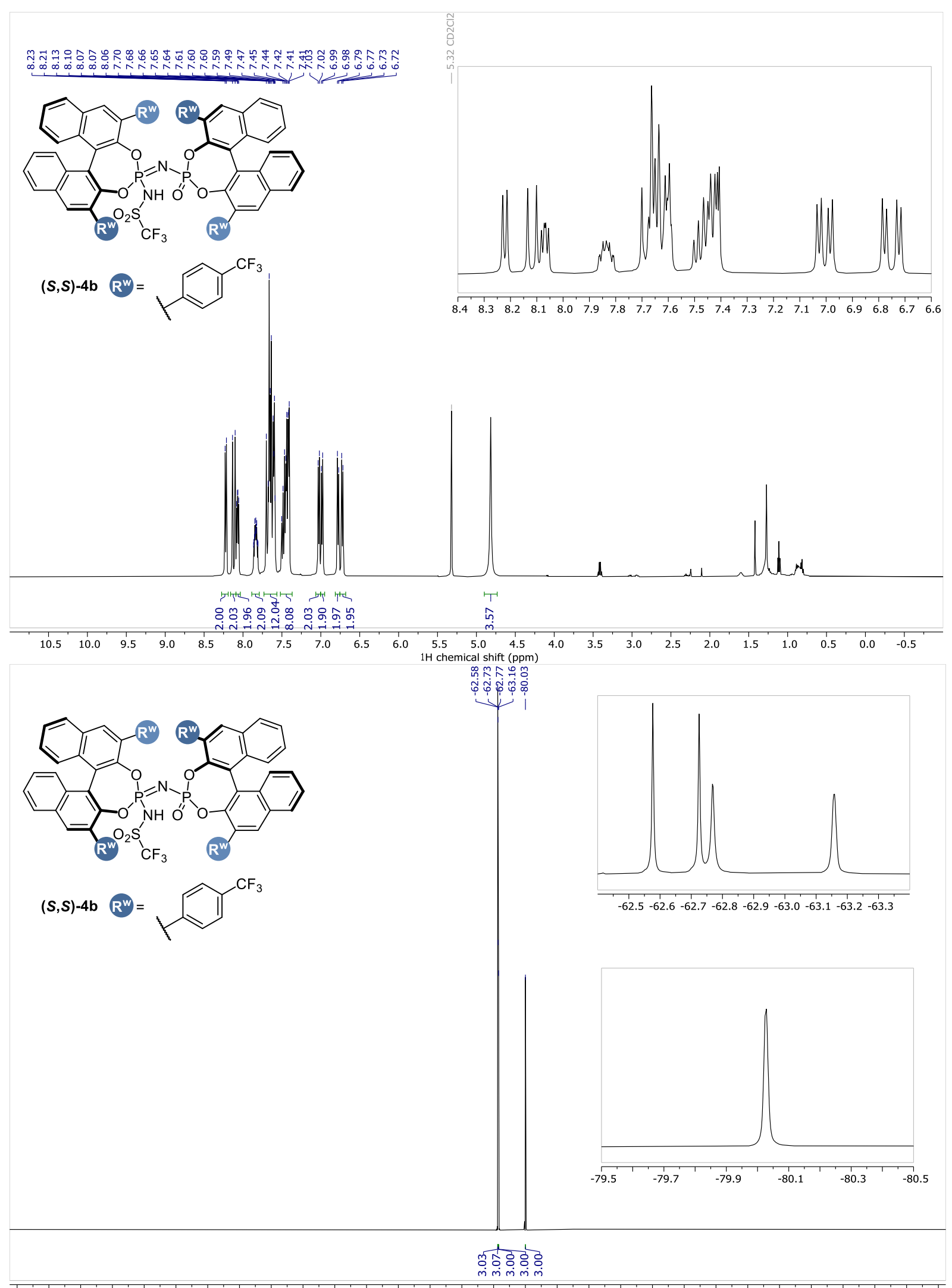
\begin{tabular}{lllllllllllllllllllllllllllllllllll}
\hline 240 & 220 & 200 & 180 & 160 & 140 & 120 & 100 & 80 & 60 & 40 & 20 & 0 & -20 & -40 & -60 & -80 & -100 & -120 & -140 & -160 & -180 & -200 & -220 & -240 & -260 & -280 & -300 & -320 & -34
\end{tabular}

NMR spectra for compound $\mathbf{4 b}:{ }^{1} \mathrm{H}(501 \mathrm{MHz})$ and ${ }^{19} \mathrm{~F}(471 \mathrm{MHz})$, in $\mathrm{CD}_{2} \mathrm{Cl}_{2}$. 

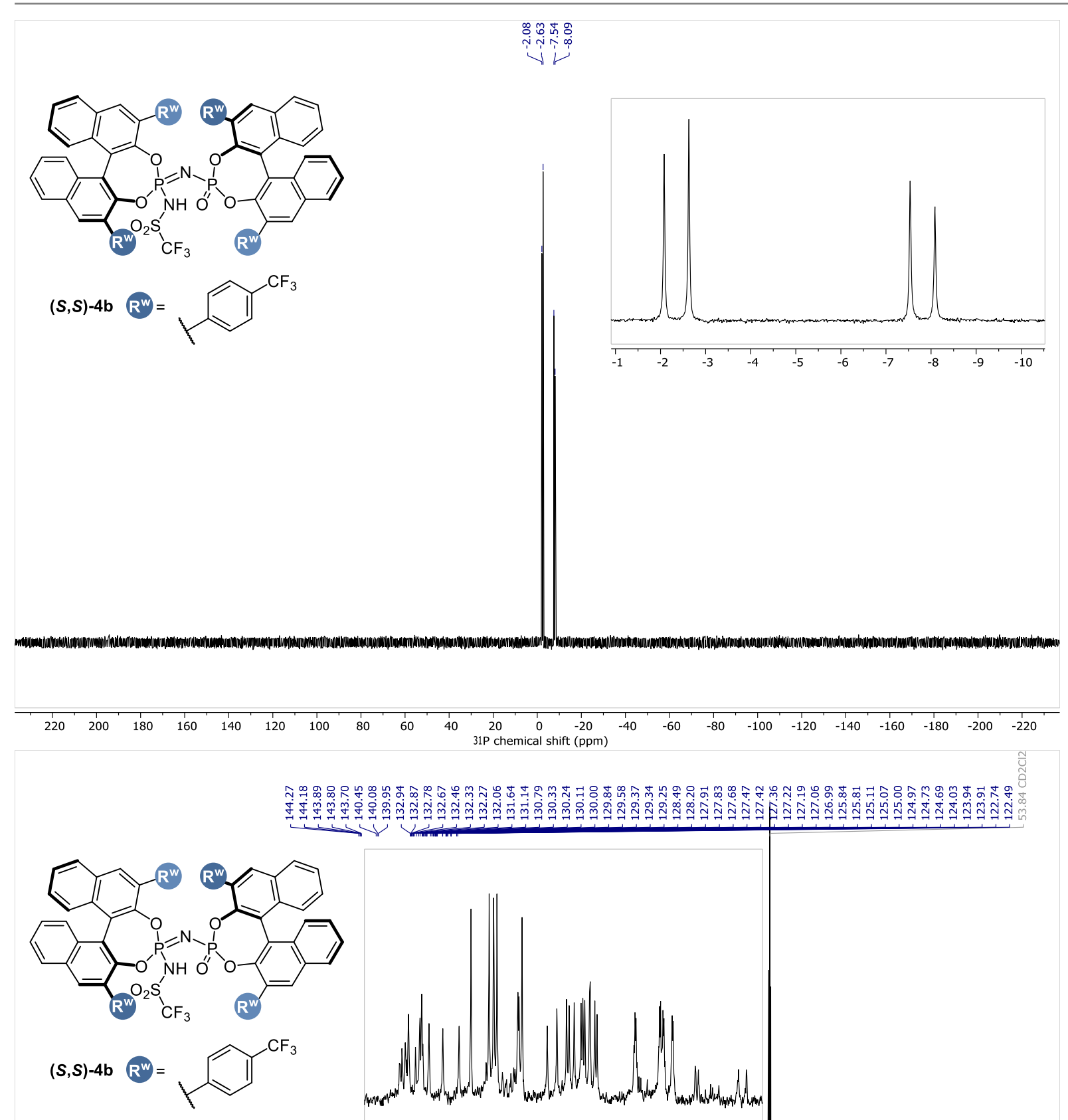

$\begin{array}{lllllllllllllll}134 & 133 & 132 & 131 & 130 & 129 & 128 & 127 & 126 & 125 & 124 & 123\end{array}$
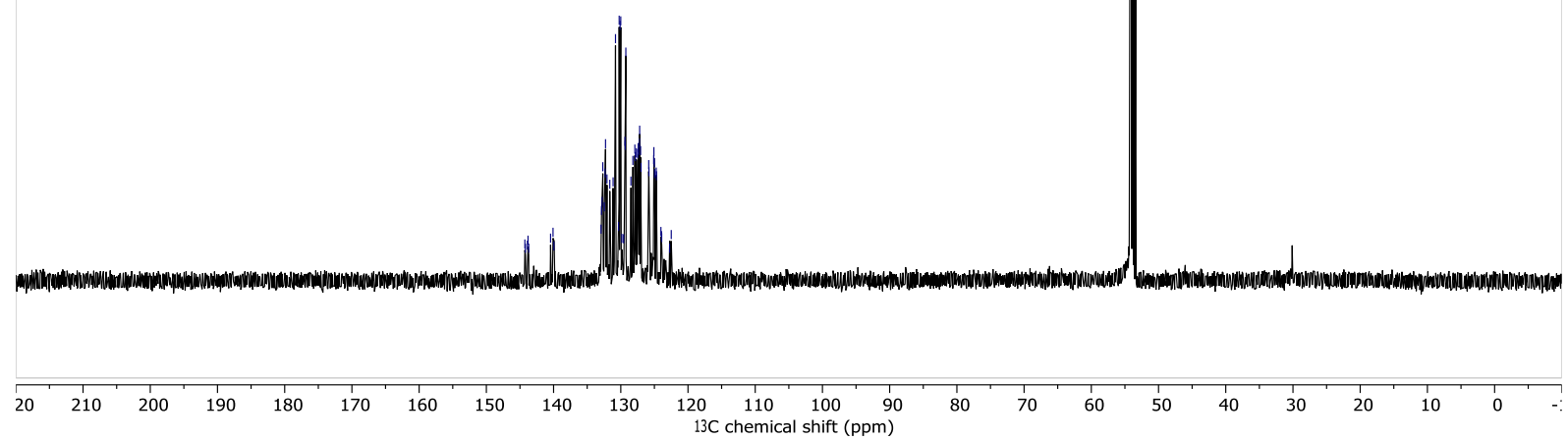

NMR spectra for compound $\mathbf{4 b}$ (continuation): ${ }^{31} \mathrm{P}(203 \mathrm{MHz})$ and ${ }^{13} \mathrm{C}(126 \mathrm{MHz})$, in $\mathrm{CD}_{2} \mathrm{Cl}_{2}$. 


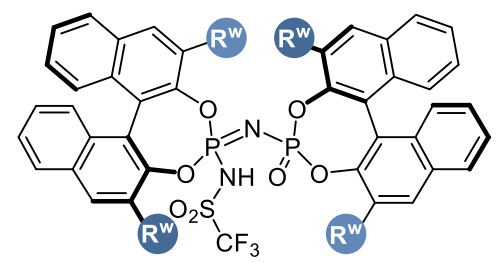

$(S, S)-4 b \quad R^{w}=$<smiles>CCc1ccc(C(F)(F)F)cc1</smiles>

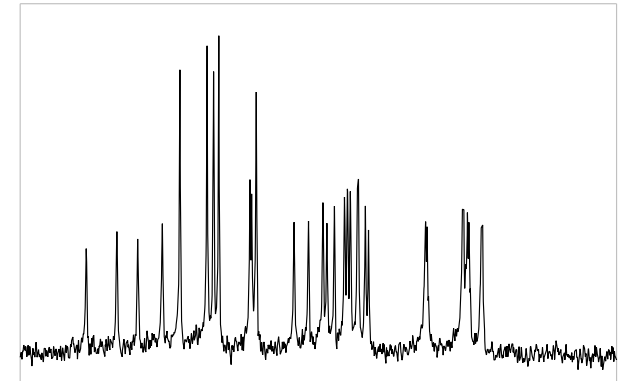
$\begin{array}{lllllllllllllllll}134 & 133 & 132 & 131 & 130 & 129 & 128 & 127 & 126 & 125 & 124 & 123 & 122\end{array}$

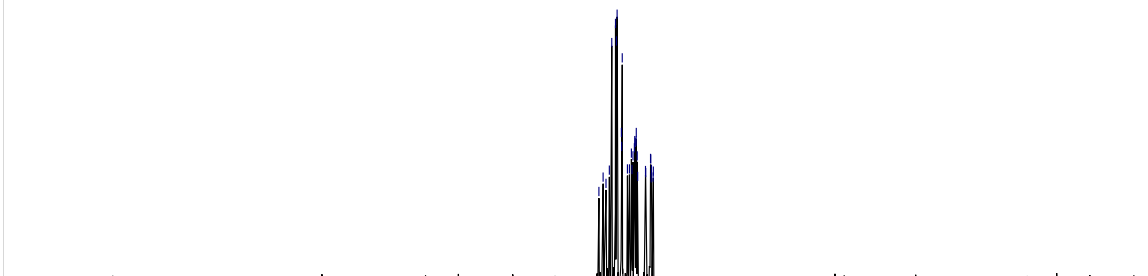

20 $\begin{array}{lllllllllllll}20 & 210 & 200 & 190 & 180 & 170 & 160 & 150 & 140 & 130 & 120 & 110 & 100 \\ 13 \mathrm{C} \text { chemical shift (ppm) } & 90\end{array}$

NMR spectra for compound 4b (continuation): DEPT-135 (126 MHz), in $\mathrm{CD}_{2} \mathrm{Cl}_{2}$. 


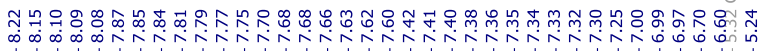

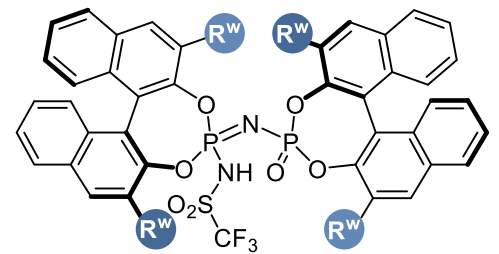

$(S, S)-4 c \quad R^{w}=$<smiles>CC(C)c1ccc(C(F)(F)F)c(Br)c1</smiles>

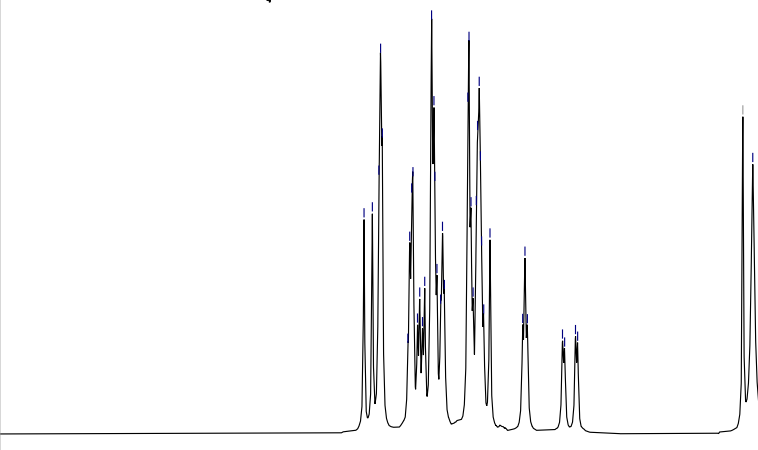

$\begin{array}{lllllllllllllllllllllllll}8.3 & 8.2 & 8.1 & 8 & 0 & 7.9 & 7.8 & 7.7 & 7.6 & 7.5 & 7.4 & 7.3 & 7.2 & 7.1 & 7.0 & 6.9 & 6.8 & 6.7 & 6.6 & 6.5\end{array}$
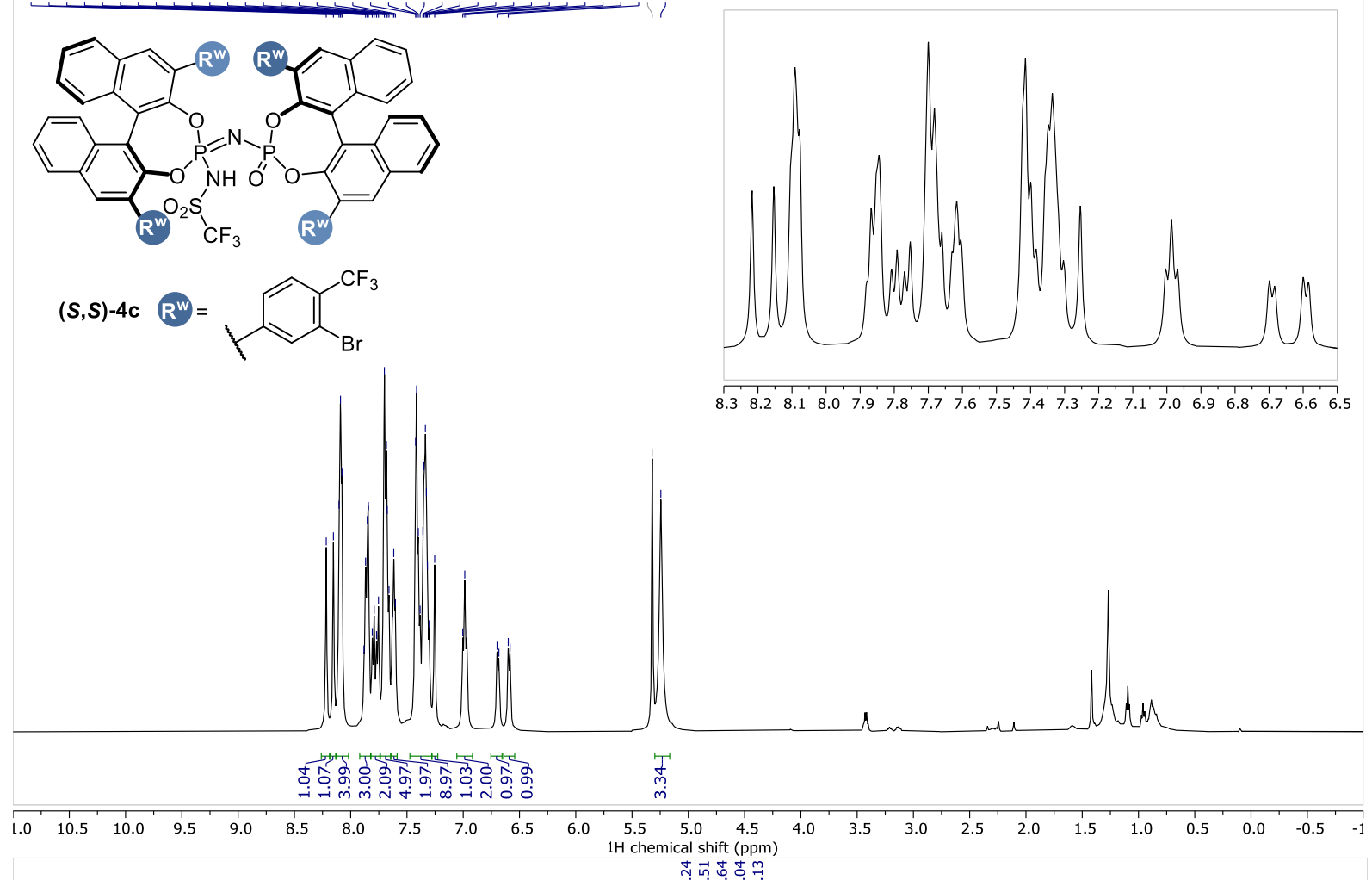
से

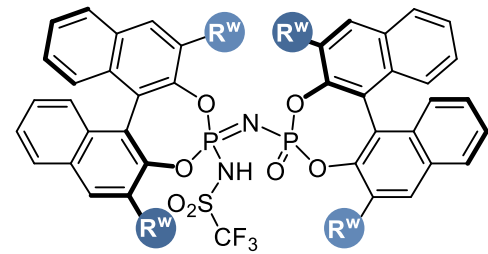

$(S, S)-4 c \quad R^{w}=$<smiles>CC(C)c1ccc(C(F)(F)F)c(Br)c1</smiles>

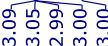

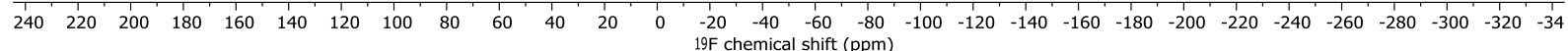

NMR spectra for compound 4c: ${ }^{1} \mathrm{H}(501 \mathrm{MHz})$ and ${ }^{19} \mathrm{~F}(471 \mathrm{MHz})$, in $\mathrm{CD}_{2} \mathrm{Cl}_{2}$. 


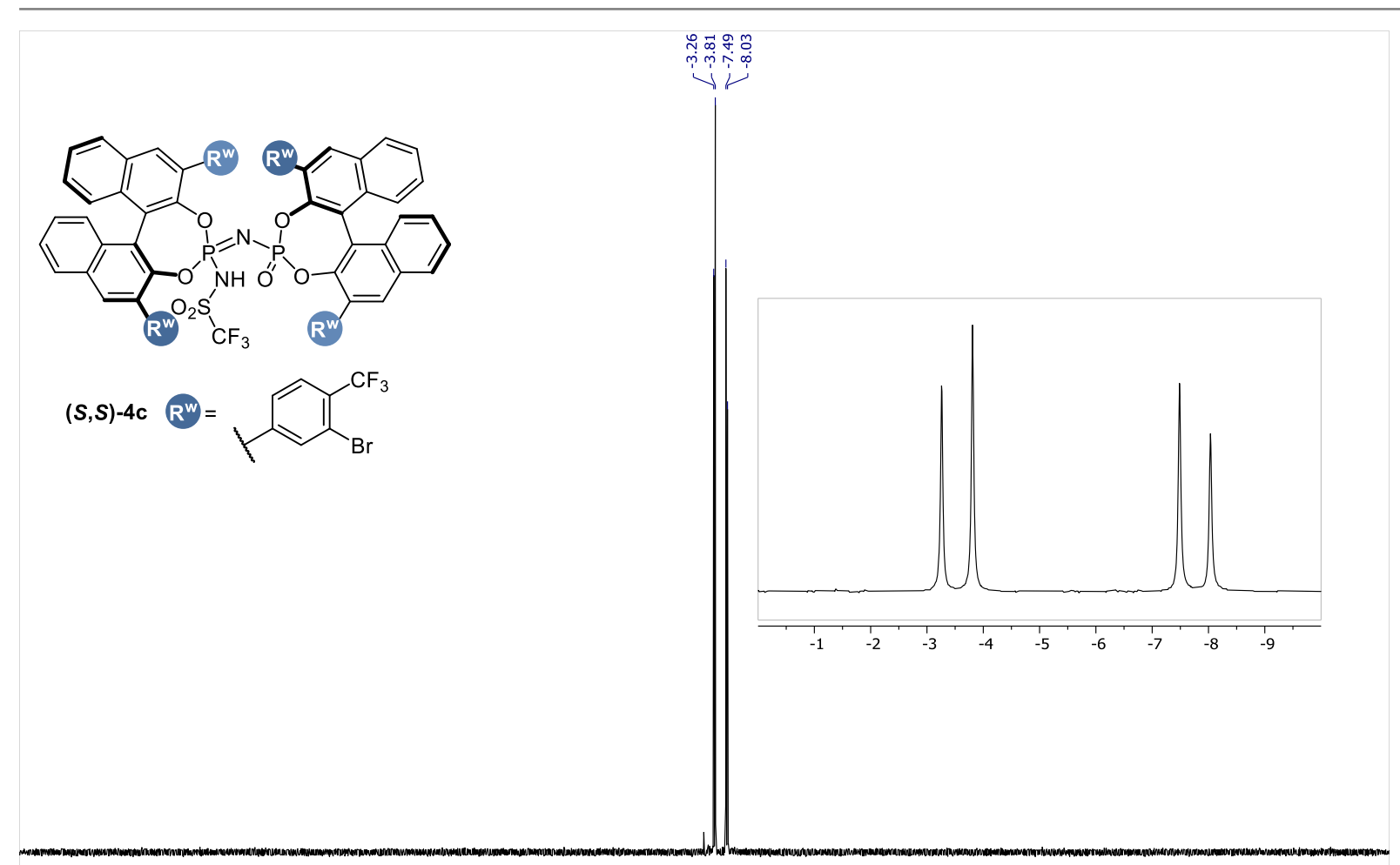

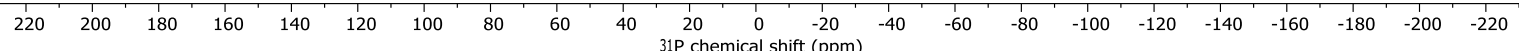

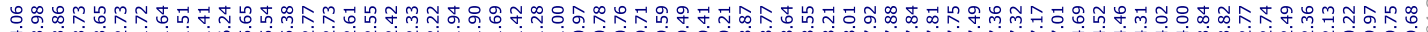

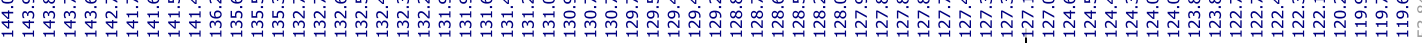

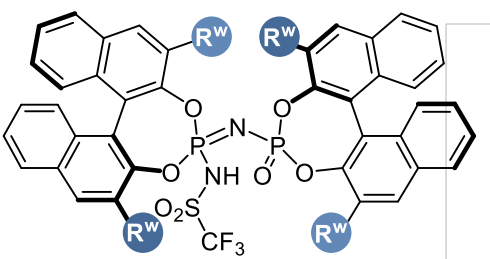

$(S, S)-4 c$<smiles>CC(C)c1ccc(C(F)(F)F)c(Br)c1</smiles>
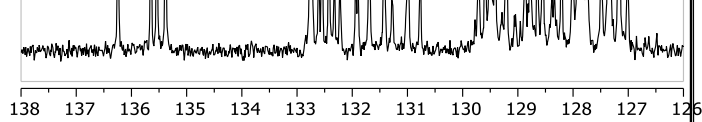

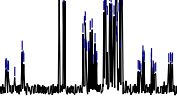

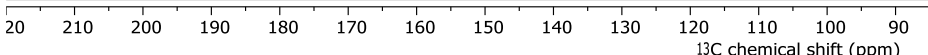

NMR spectra for compound 4c (continuation): ${ }^{31} \mathrm{P}(203 \mathrm{MHz})$ and ${ }^{13} \mathrm{C}(126 \mathrm{MHz})$, in $\mathrm{CD}_{2} \mathrm{Cl}_{2}$. 


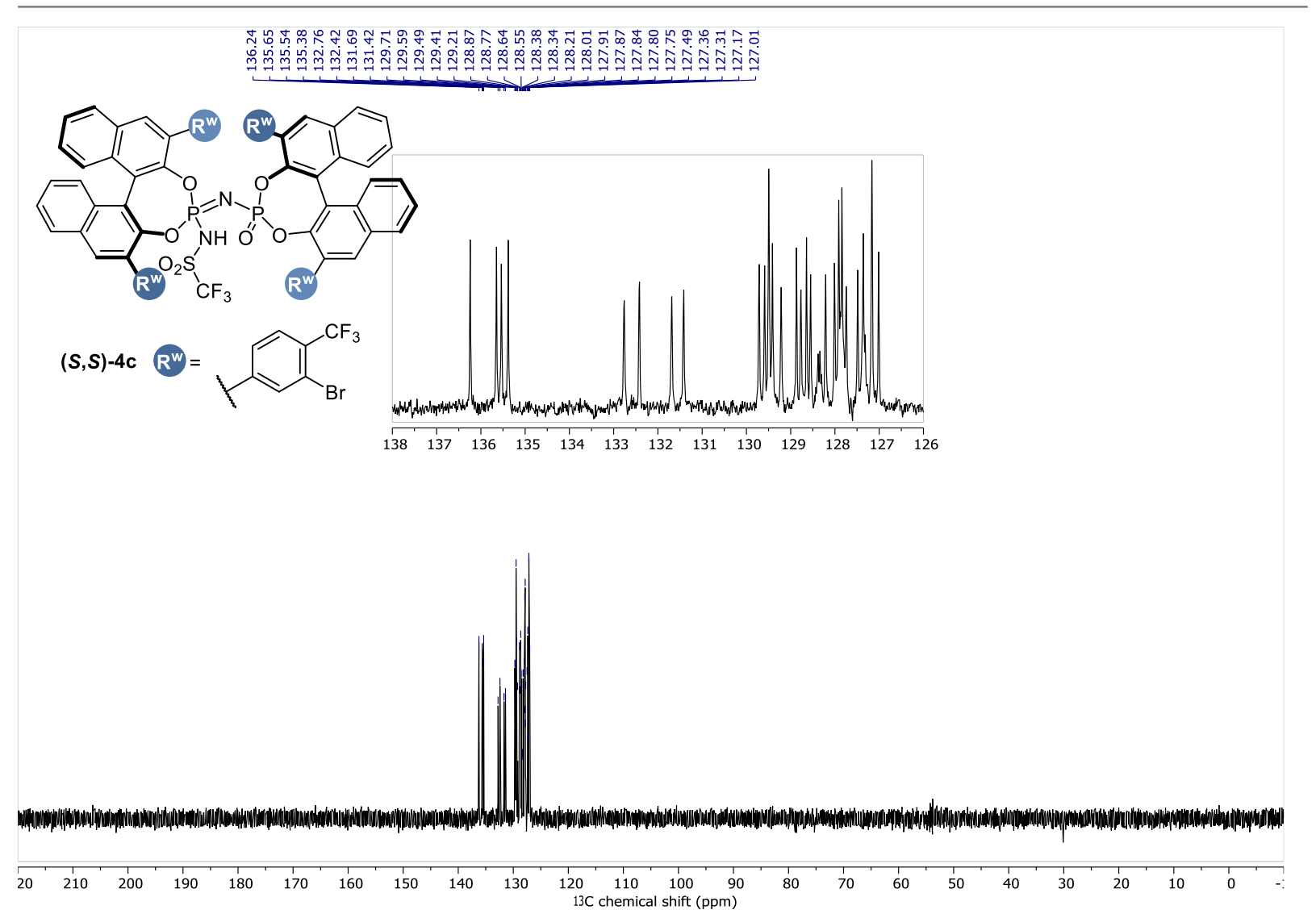

NMR spectra for compound 4c (continuation): DEPT-135 (126 MHz), in $\mathrm{CD}_{2} \mathrm{Cl}_{2}$. 


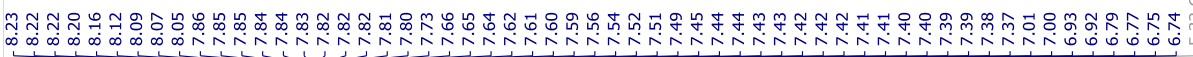

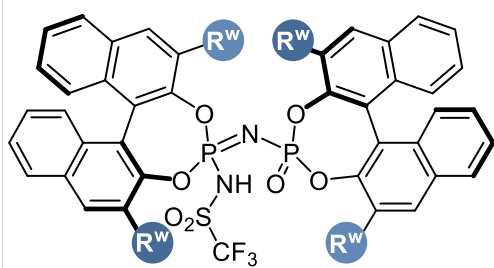

$(s, S)-4 \mathrm{~d} \quad \mathrm{R}^{\mathrm{w}}=$

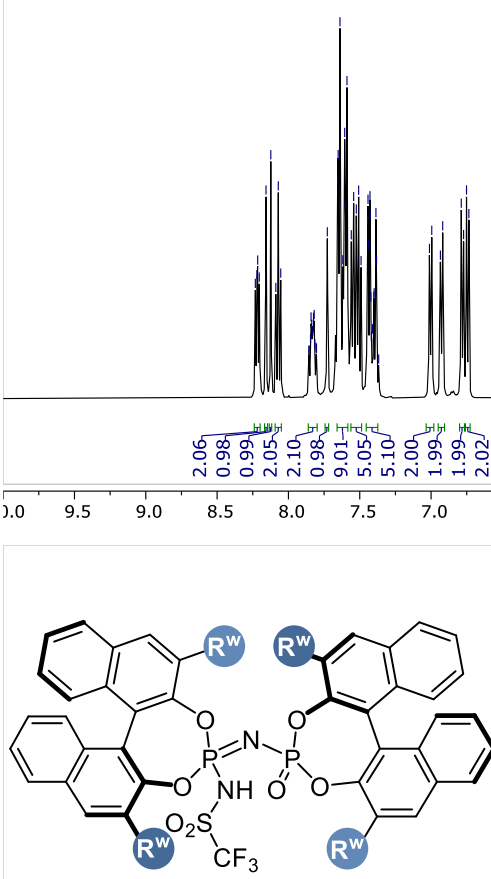

$(s, S)-4 d R^{w}=$

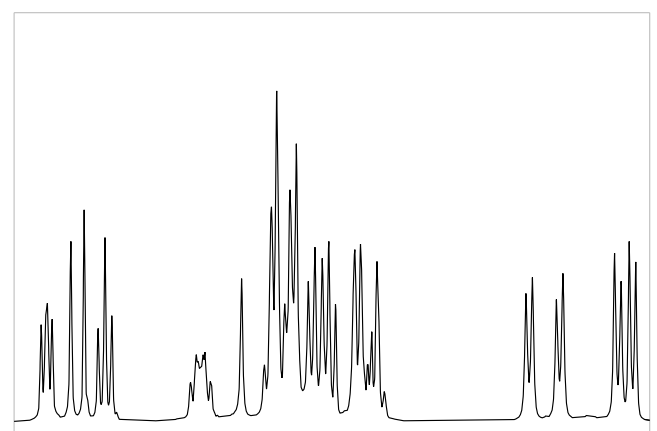

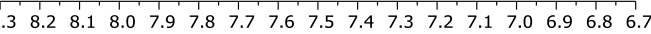

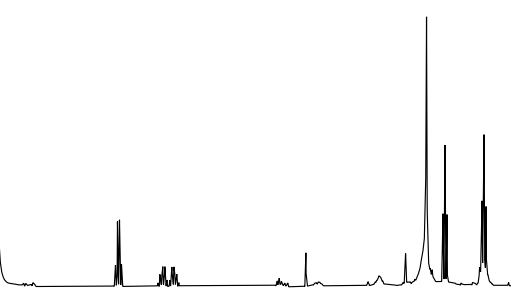



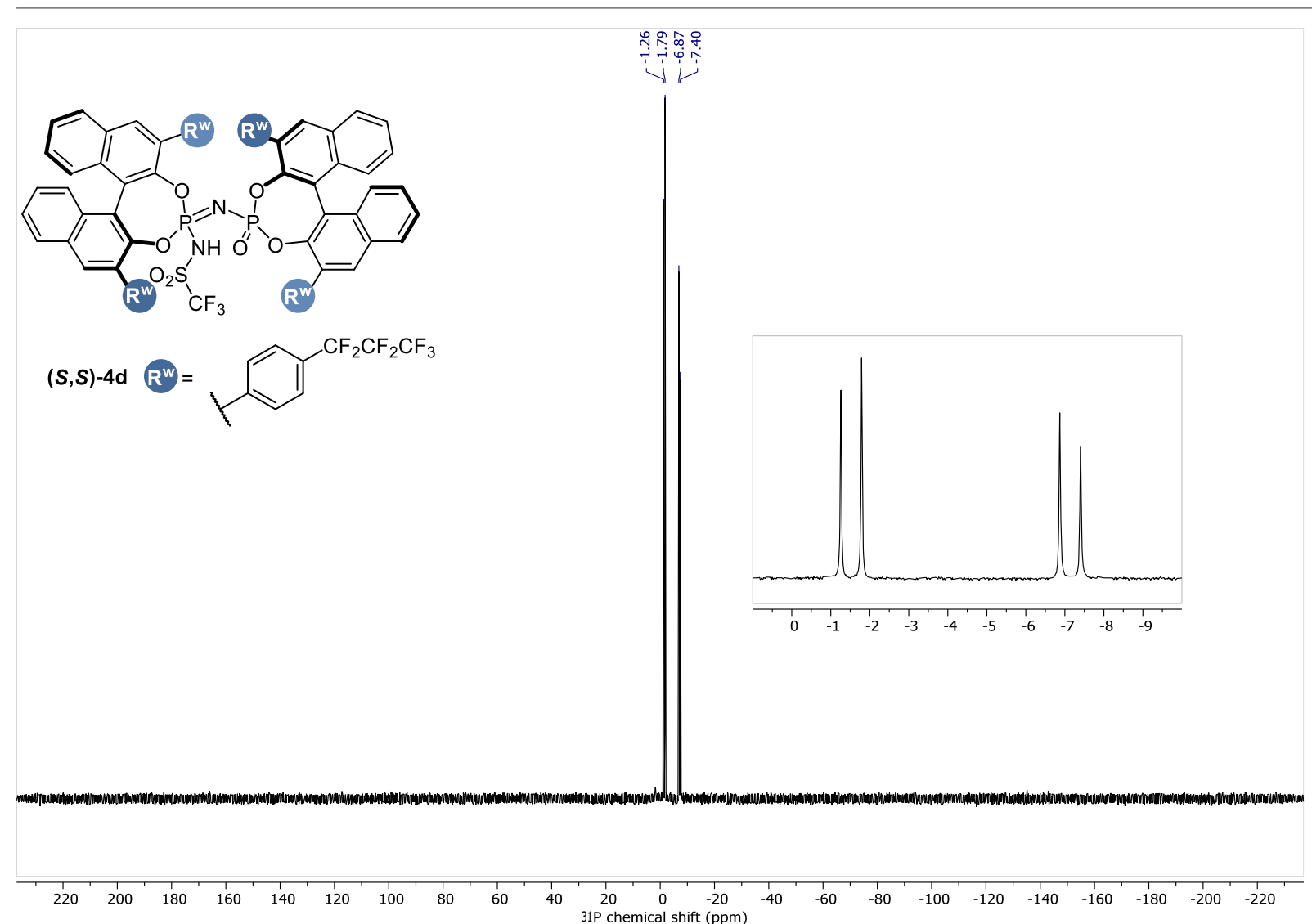

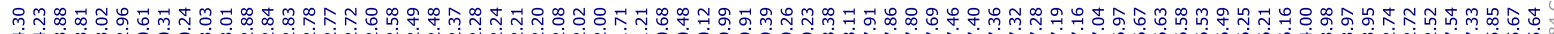

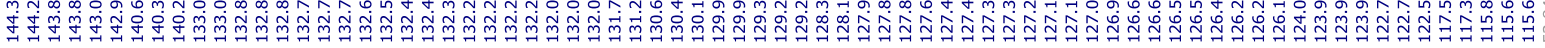

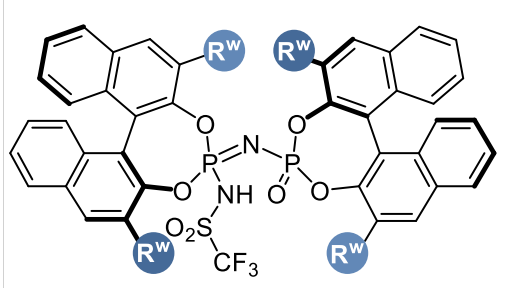

$(S, S)-4 d \quad R^{W}=$<smiles>CC(C)(C)c1ccc(C(F)(F)C(F)(F)F)cc1</smiles>

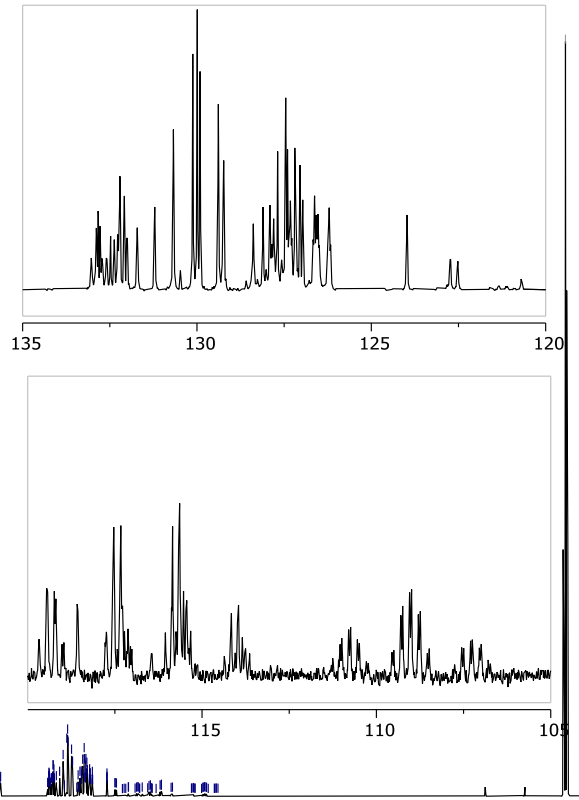

$\begin{array}{lllllllllllll}210 & 200 & 190 & 180 & 170 & 160 & 150 & 140 & 130 & 120 & 110 & 100 & 90 \\ 13 \mathrm{C} \text { chemical shift (ppm) }\end{array}$

NMR spectra for compound 4d (continuation): ${ }^{31} \mathrm{P}(203 \mathrm{MHz})$, and ${ }^{13} \mathrm{C}(151 \mathrm{MHz})$, in $\mathrm{CD}_{2} \mathrm{Cl}_{2}$. 


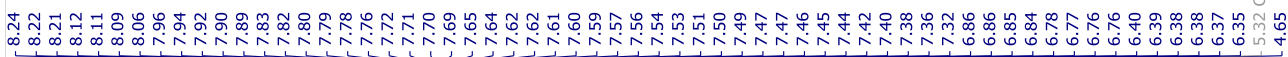
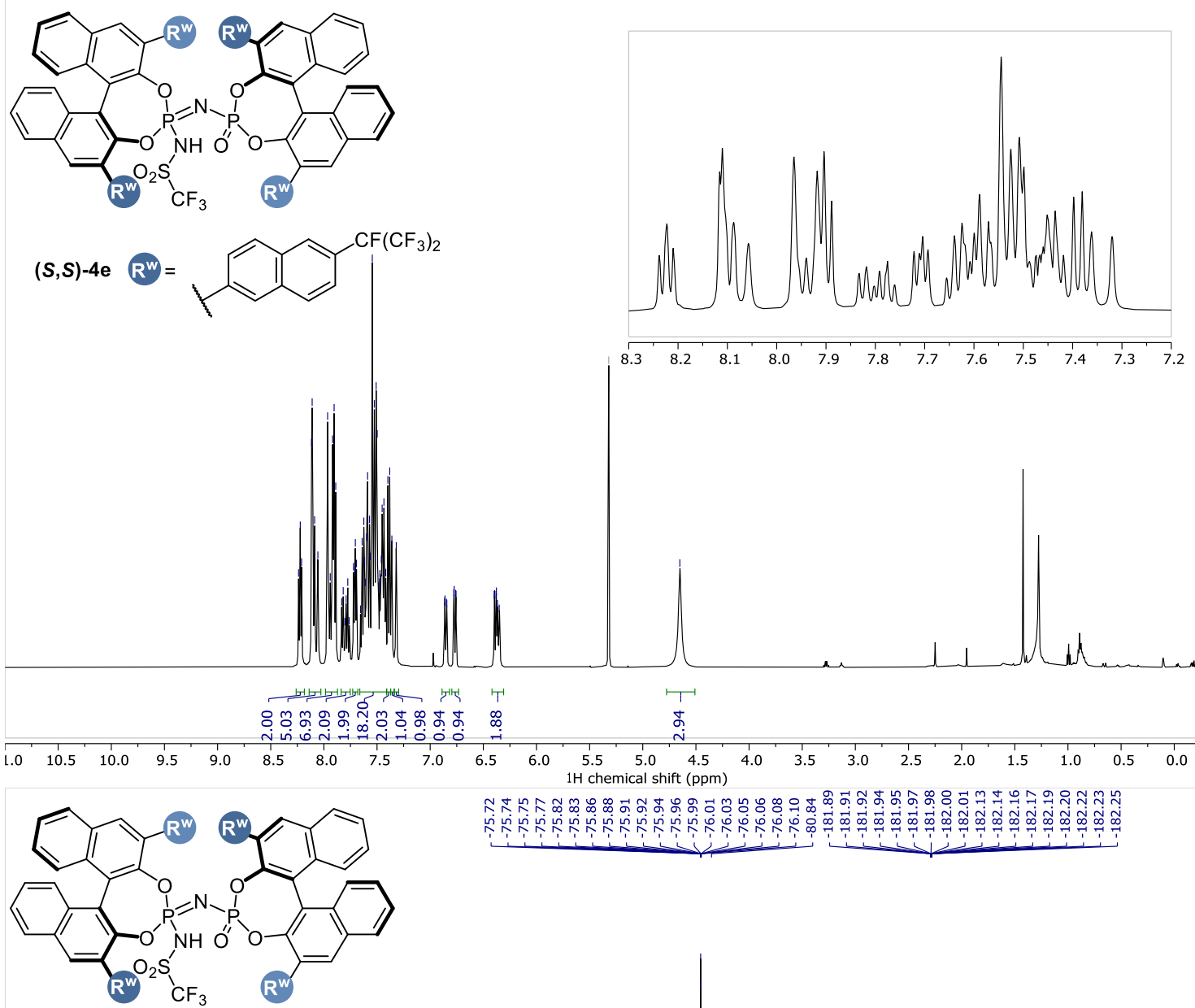

$(S, S)-4$<smiles>CC(C)c1ccc2cc(C(C)C(C)(C)C)ccc2c1</smiles>
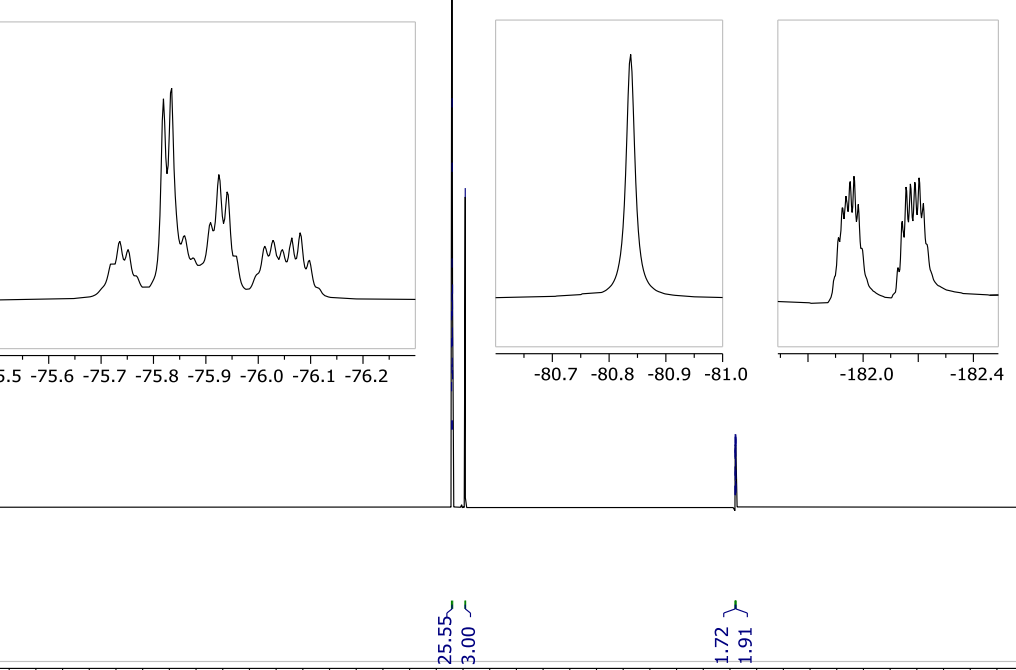

ᄉิ-

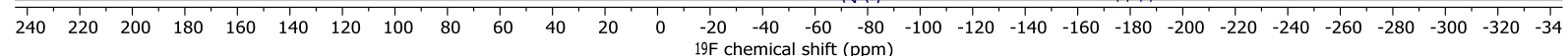

NMR spectra for compound $4 \mathrm{e}:{ }^{1} \mathrm{H}(501 \mathrm{MHz})$ and ${ }^{19} \mathrm{~F}(471 \mathrm{MHz})$, in $\mathrm{CD}_{2} \mathrm{Cl}_{2}$. 

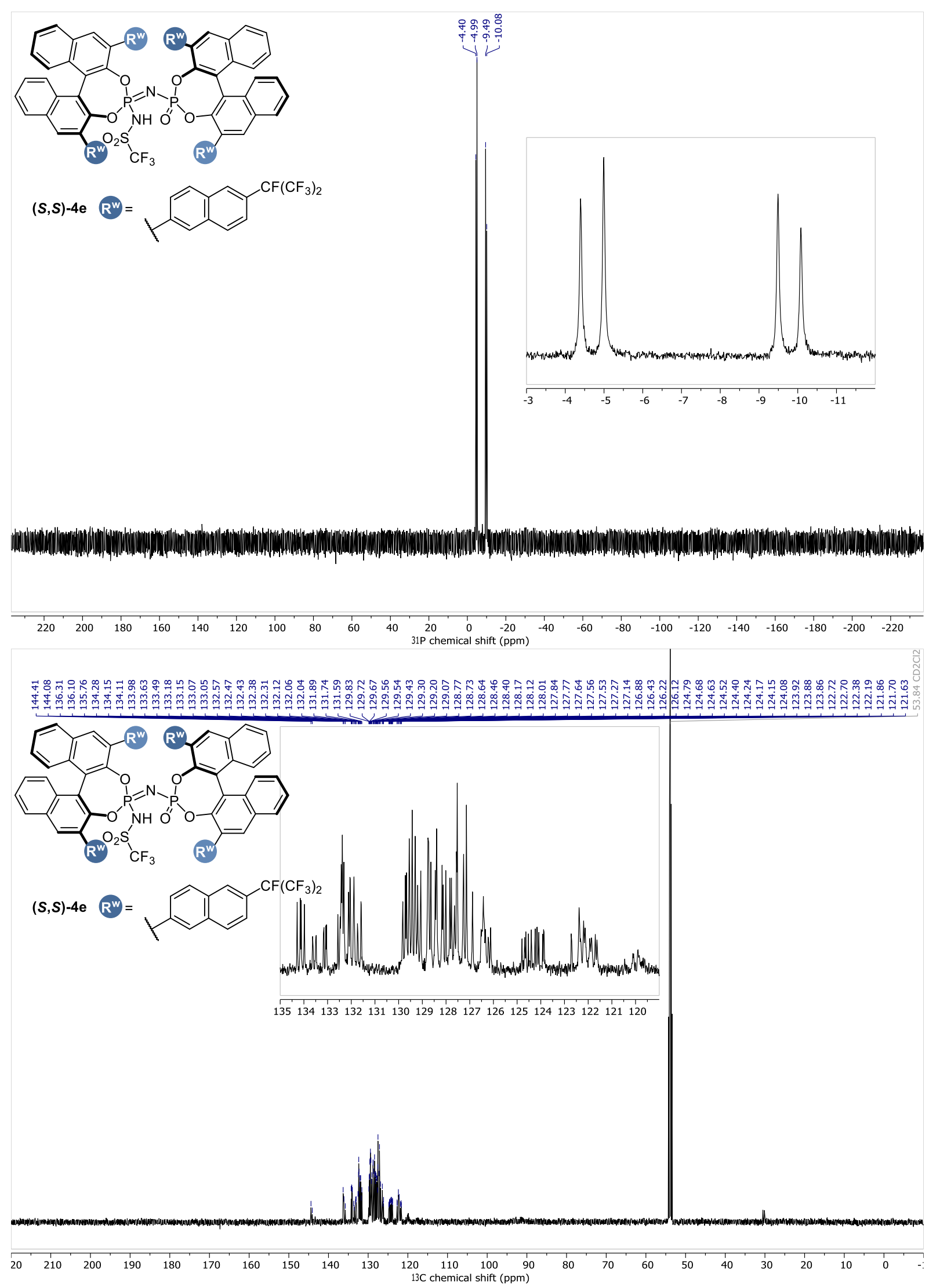

NMR spectra for compound $4 \mathrm{e}$ (continuation): ${ }^{31} \mathrm{P}(203 \mathrm{MHz})$ and ${ }^{13} \mathrm{C}(126 \mathrm{MHz})$, in $\mathrm{CD}_{2} \mathrm{Cl}_{2}$. 
The Catalytic Asymmetric Intermolecular Prins Reaction Copies of NMR spectra

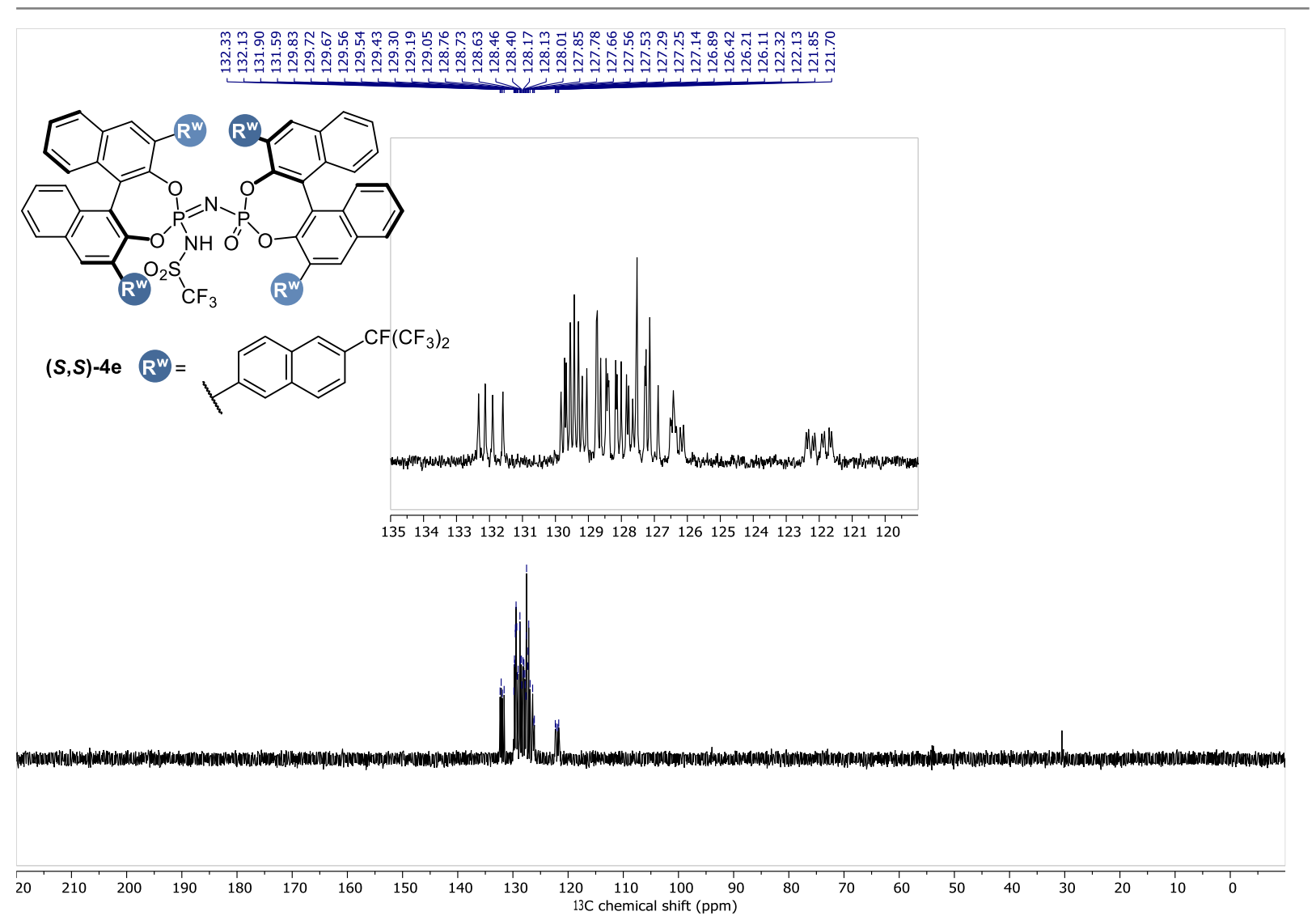

NMR spectra for compound $4 \mathbf{e}$ (continuation): DEPT-135 (126 MHz), in $\mathrm{CD}_{2} \mathrm{Cl}_{2}$. 


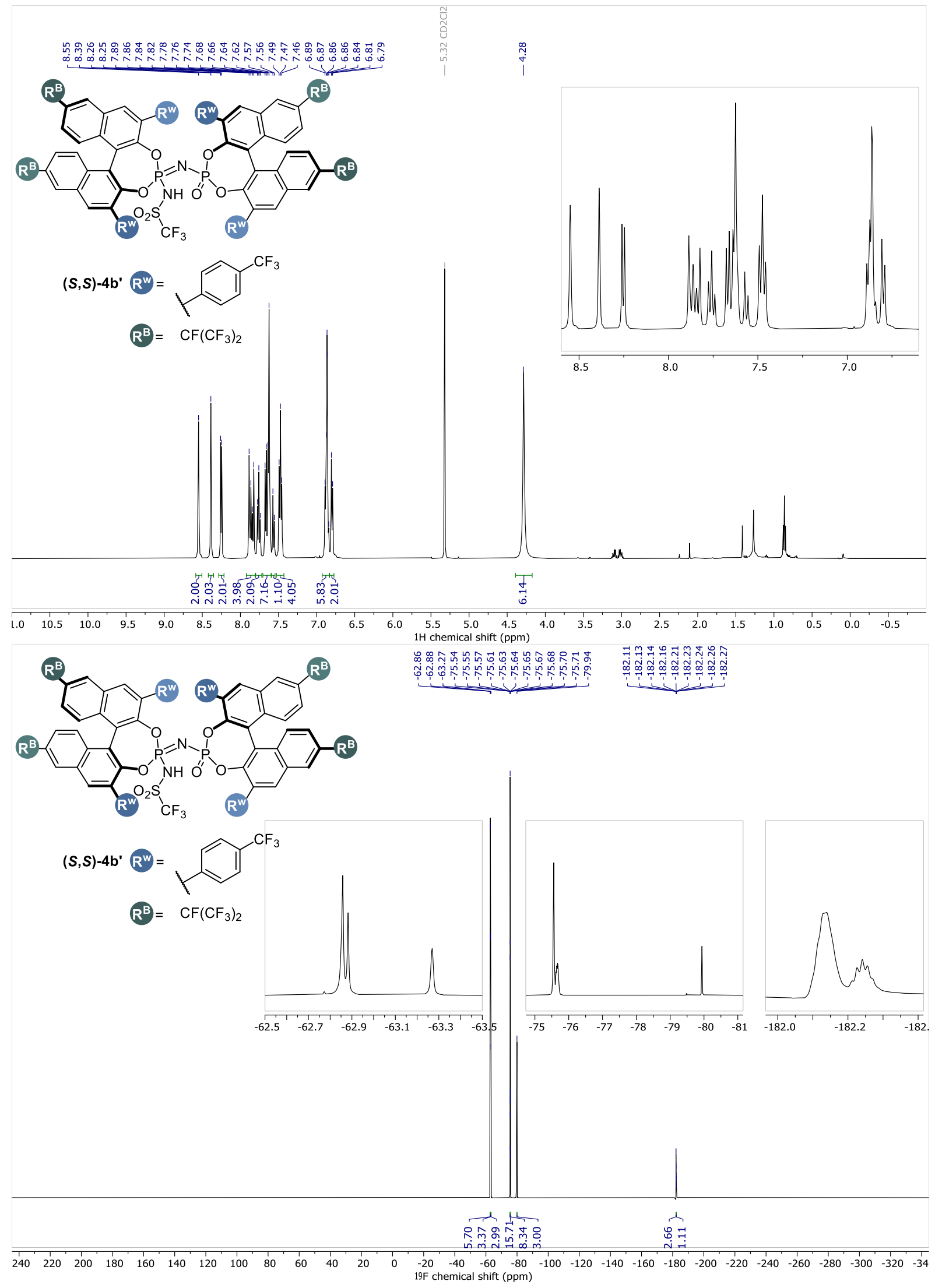

NMR spectra for compound $4 \mathbf{b}^{\prime}:{ }^{1} \mathrm{H}(501 \mathrm{MHz})$ and ${ }^{19} \mathrm{~F}(471 \mathrm{MHz})$, in $\mathrm{CD}_{2} \mathrm{Cl}_{2}$. 

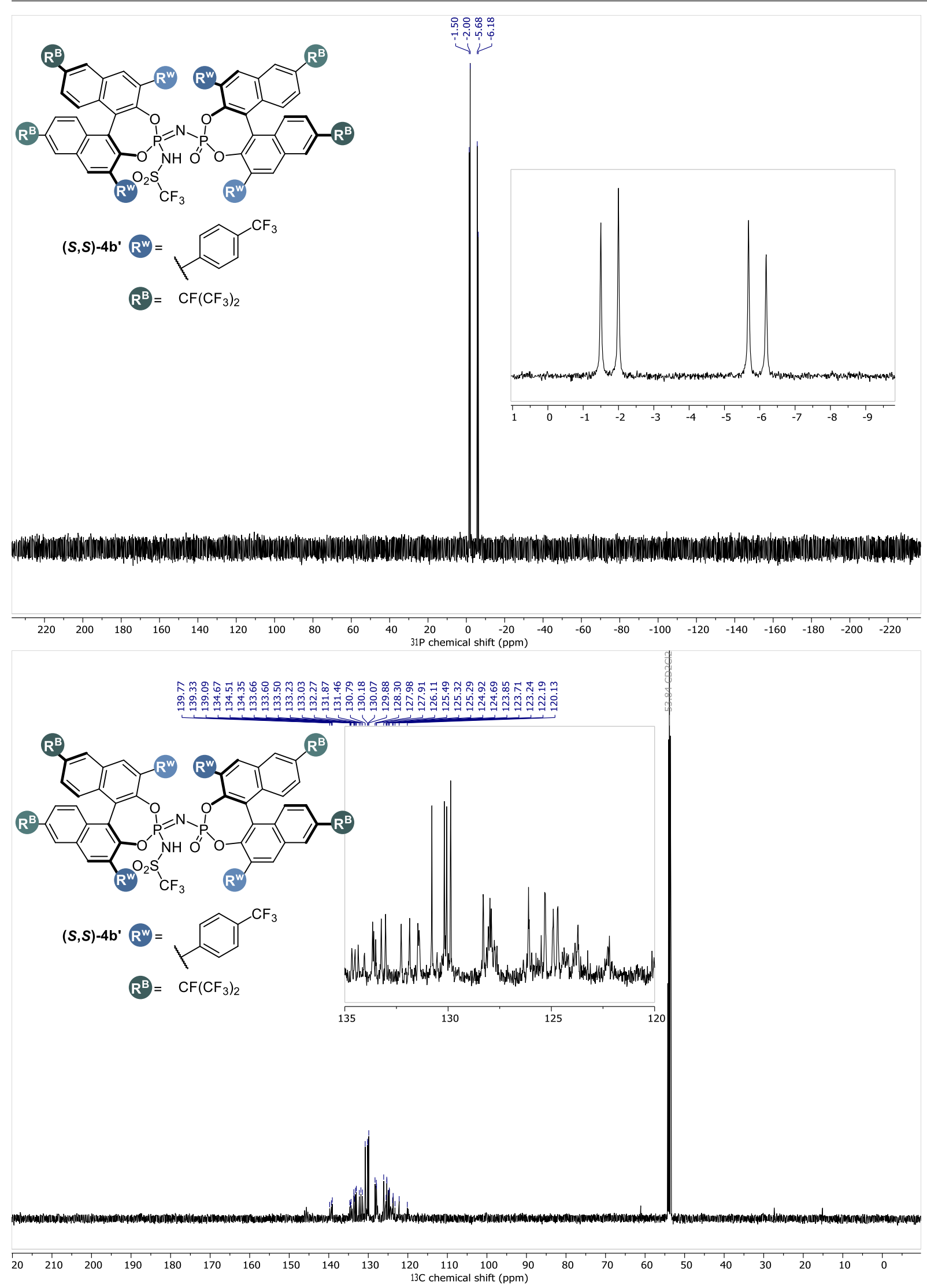

NMR spectra for compound 4b' (continuation): ${ }^{31} \mathrm{P}(203 \mathrm{MHz})$ and ${ }^{13} \mathrm{C}(126 \mathrm{MHz})$, in $\mathrm{CD}_{2} \mathrm{Cl}_{2}$. 


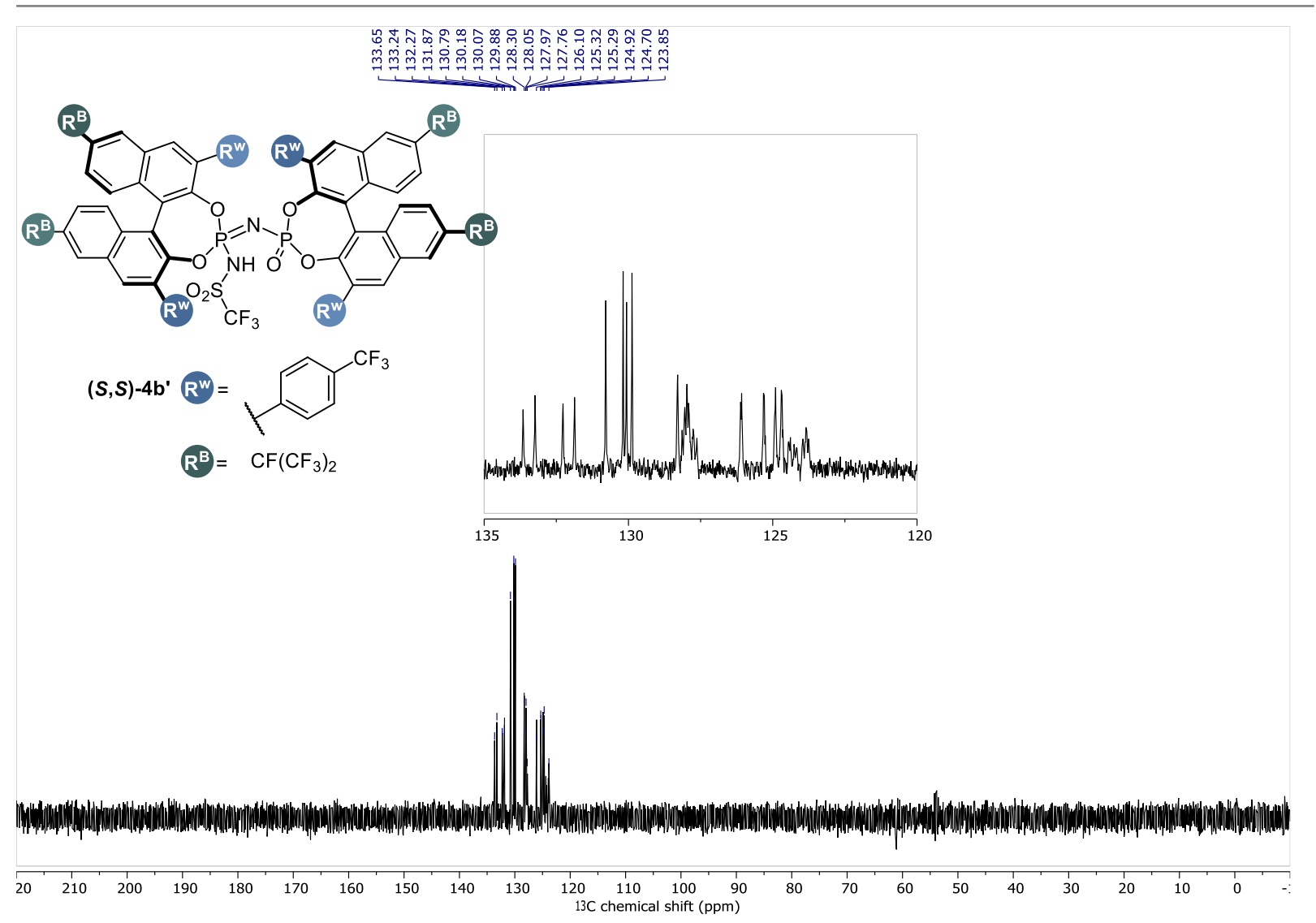

NMR spectra for compound 4b' (continuation): DEPT-135 (126 MHz), in $\mathrm{CD}_{2} \mathrm{Cl}_{2}$. 
The Catalytic Asymmetric Intermolecular Prins Reaction

Copies of HPLC traces

\section{Copies of HPLC traces}

(Chiralpak IB-3 column, Heptane/i-PrOH 95:5, 1 mL/min, $25^{\circ} \mathrm{C}, 220 \mathrm{~nm}$ )

$\mathrm{mAU}$

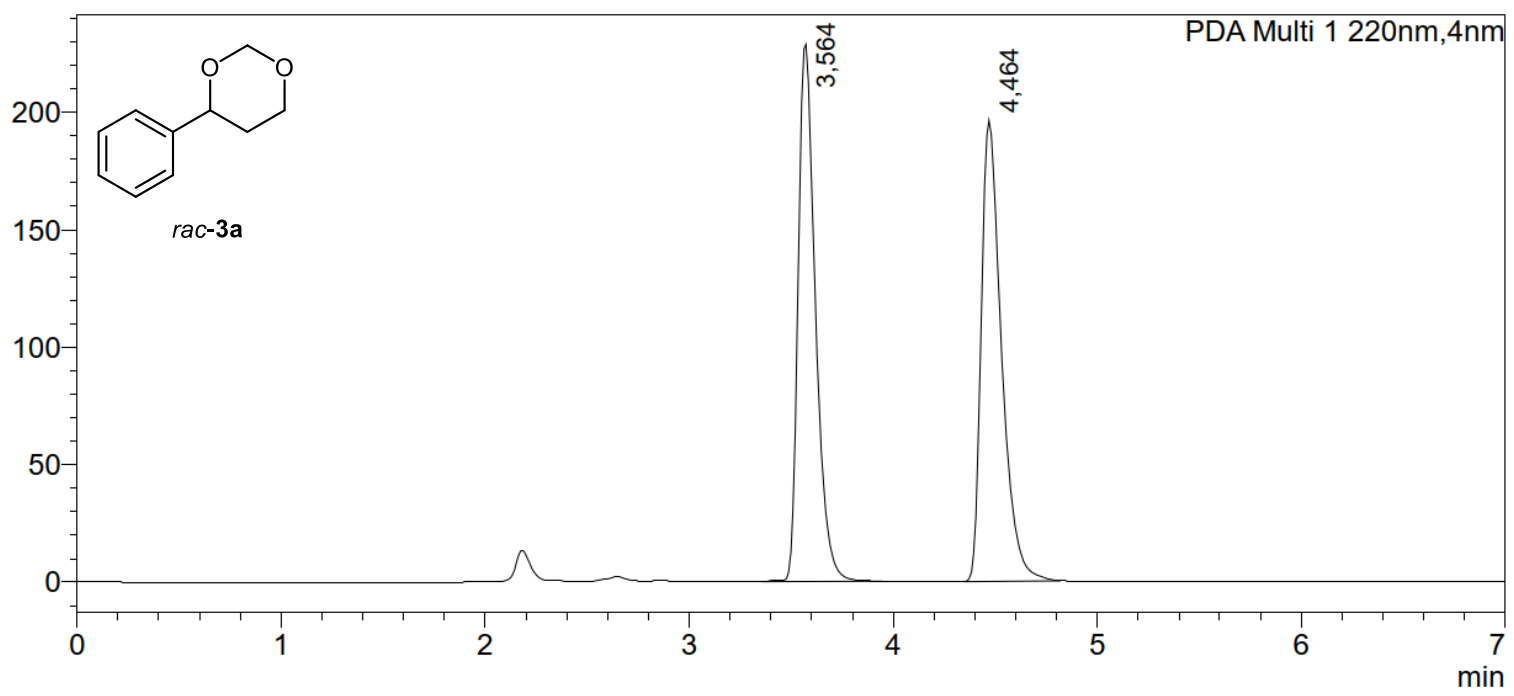

\begin{tabular}{|r|r|r|}
\hline Peak\# & Ret. Time & \multicolumn{1}{c|}{ Area\% } \\
\hline 1 & 3,564 & 49,886 \\
\hline 2 & 4,464 & 50,114 \\
\hline Total & & 100,000 \\
\hline
\end{tabular}

mAU

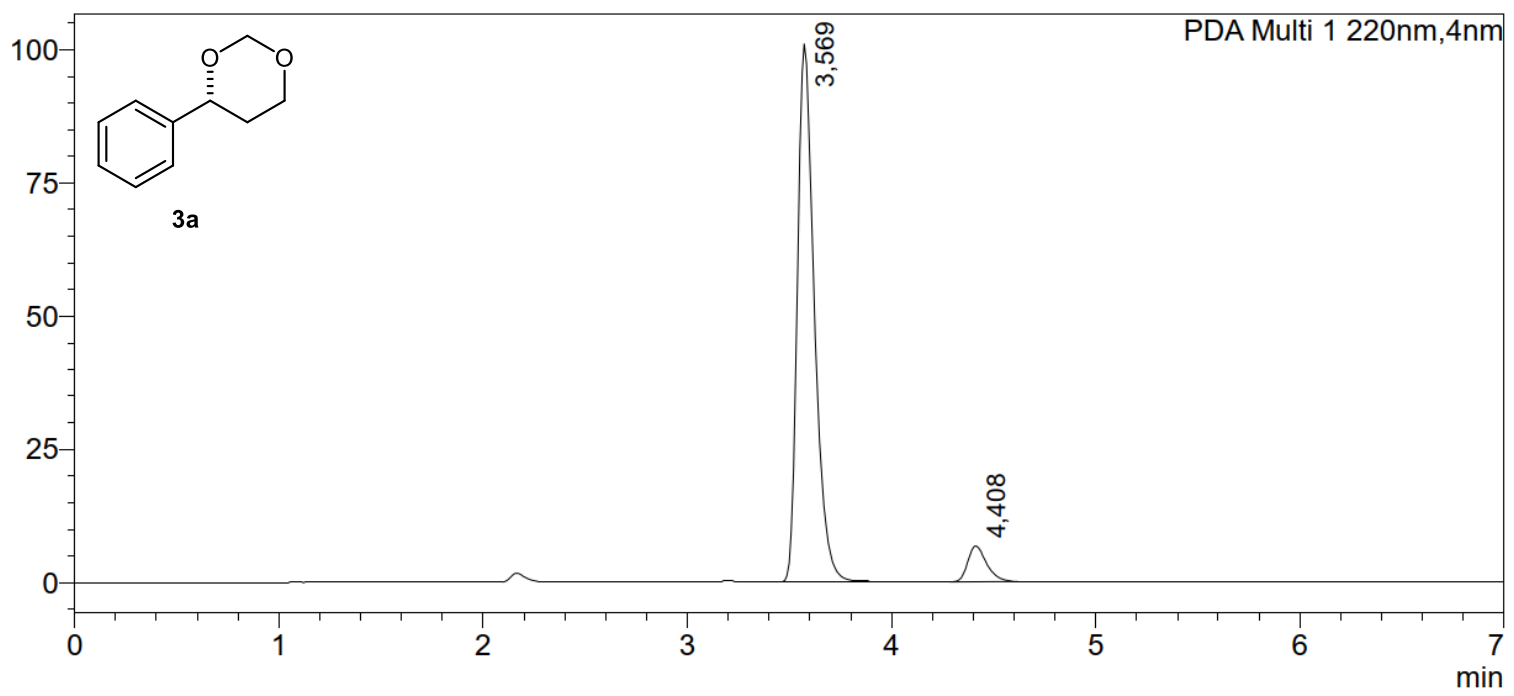

\begin{tabular}{|r|r|r|}
\hline Peak\# & Ret. Time & \multicolumn{1}{|c|}{ Area\% } \\
\hline 1 & 3,569 & 95,421 \\
\hline 2 & 4,408 & 4,579 \\
\hline Total & & 100,000 \\
\hline
\end{tabular}


The Catalytic Asymmetric Intermolecular Prins Reaction Copies of HPLC traces

(Chiralpak IC-3 column, Heptane/i-PrOH 98:2, 1 mL/min, $25^{\circ} \mathrm{C}, 220 \mathrm{~nm}$ )

mAU

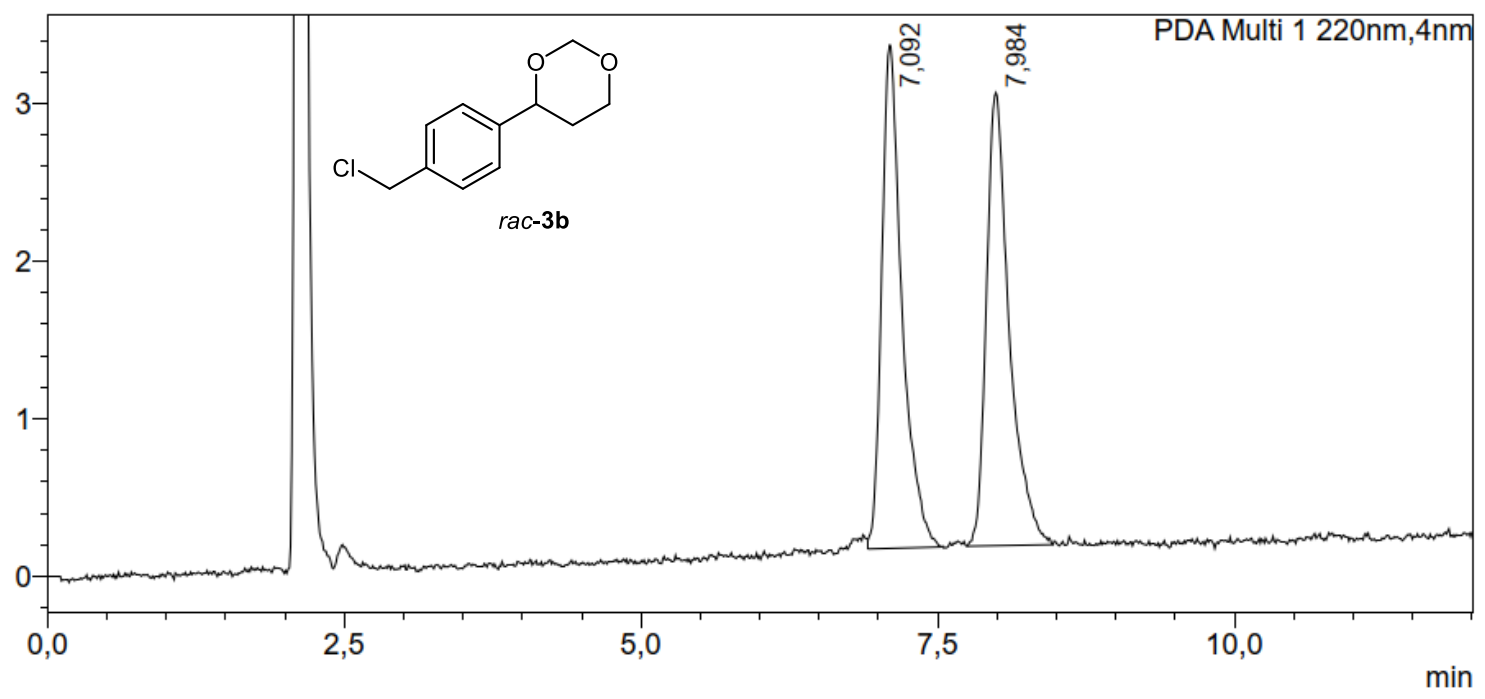

\begin{tabular}{|r|r|r|}
\hline Peak\# & Ret. Time & \multicolumn{1}{c|}{ Area\% } \\
\hline 1 & 7,092 & 49,583 \\
\hline 2 & 7,984 & 50,417 \\
\hline Total & & 100,000 \\
\hline
\end{tabular}

mAU

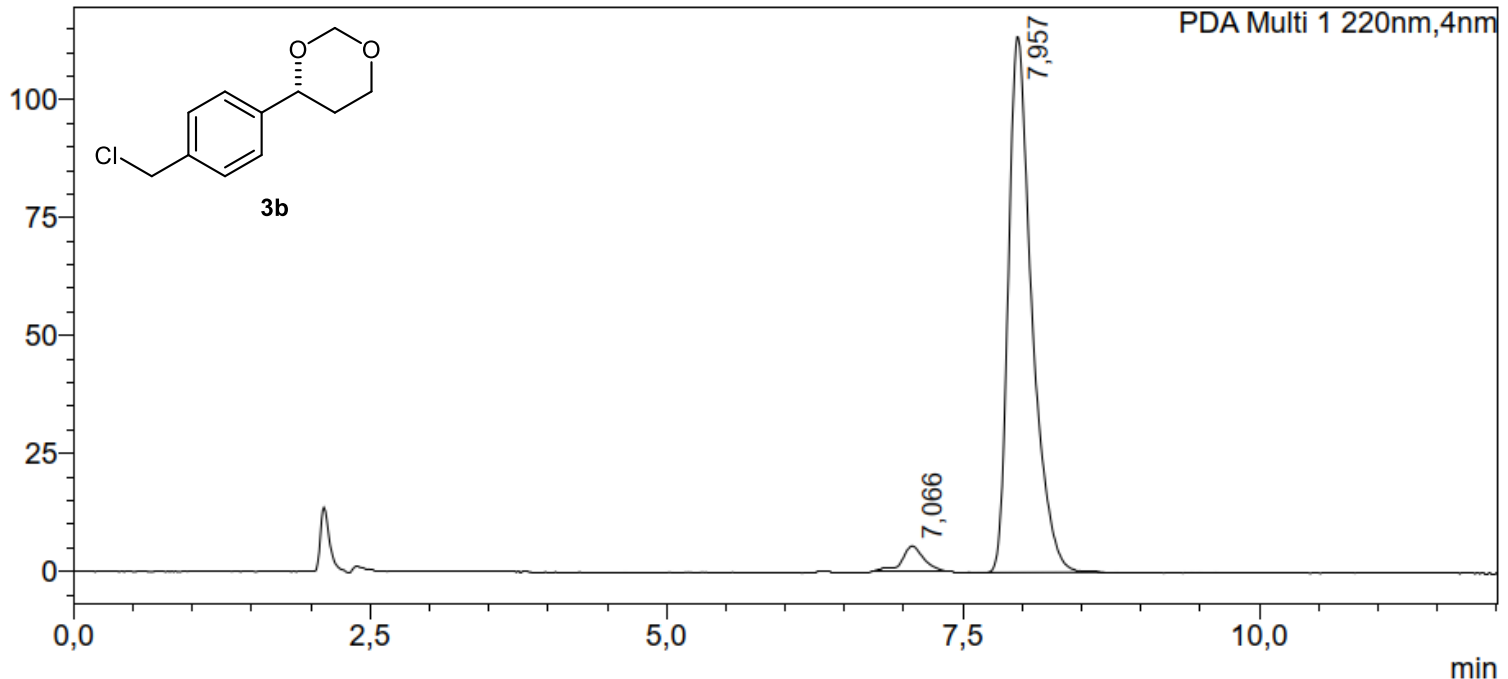

\begin{tabular}{|r|r|r|}
\hline Peak\# & Ret. Time & \multicolumn{1}{c|}{ Area\% } \\
\hline 1 & 7,066 & 4,551 \\
\hline 2 & 7,957 & 95,449 \\
\hline Total & & 100,000 \\
\hline
\end{tabular}


The Catalytic Asymmetric Intermolecular Prins Reaction Copies of HPLC traces

(Chiralpak AD-3 column, Heptane/i-PrOH 97:3, 0.5 mL/min, $25^{\circ} \mathrm{C}, 220 \mathrm{~nm}$ )

mAU

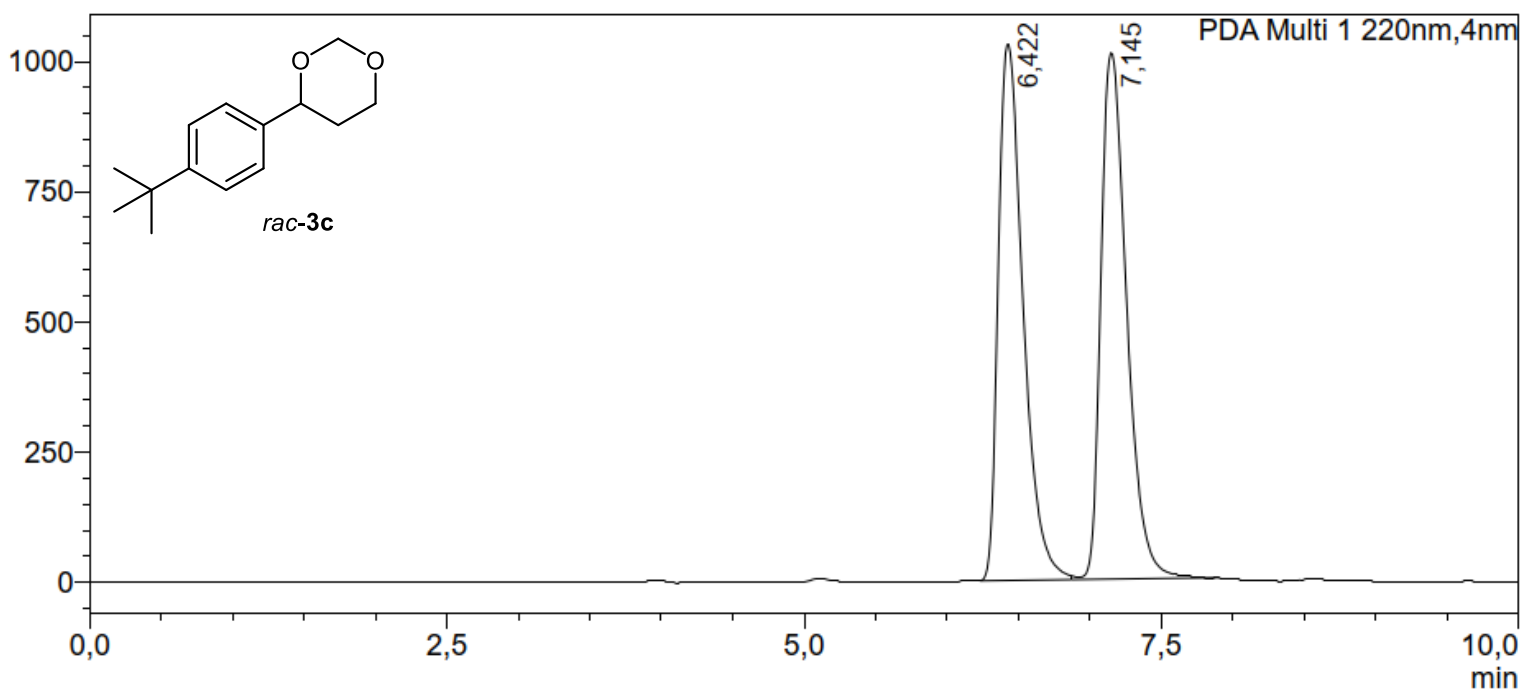

\begin{tabular}{|r|r|r|}
\hline Peak\# & Ret. Time & \multicolumn{1}{c|}{ Area\% } \\
\hline 1 & 6,422 & 49,484 \\
\hline 2 & 7,145 & 50,516 \\
\hline Total & & 100,000 \\
\hline
\end{tabular}

mAU

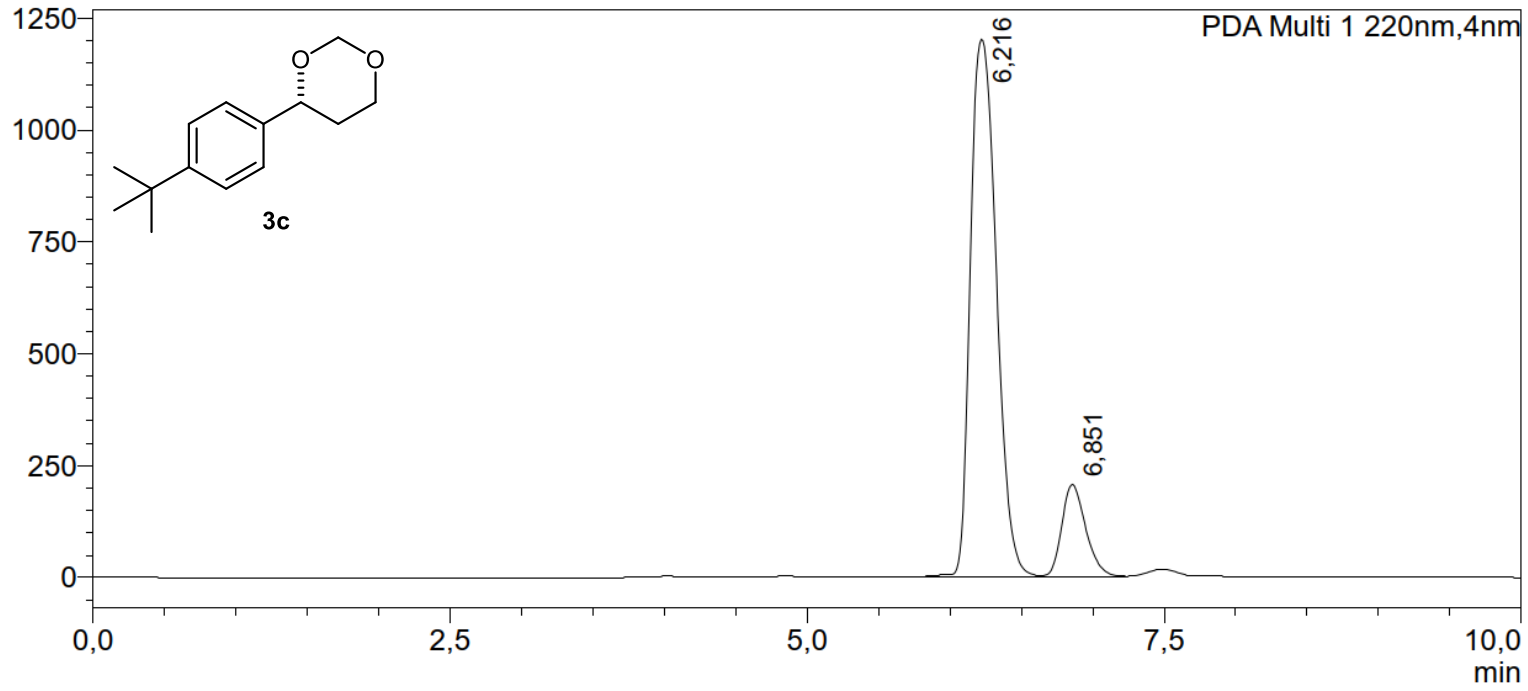

\begin{tabular}{|r|r|r|}
\hline Peak\# & Ret. Time & \multicolumn{1}{c|}{ Area\% } \\
\hline 1 & 6,216 & 91,308 \\
\hline 2 & 6,851 & 8,692 \\
\hline Total & & 100,000 \\
\hline
\end{tabular}


The Catalytic Asymmetric Intermolecular Prins Reaction Copies of HPLC traces

(Chiralpak IB-3 column, Heptane/i-PrOH 98:2, 1 mL/min, $25^{\circ} \mathrm{C}, 220 \mathrm{~nm}$ )

mAU

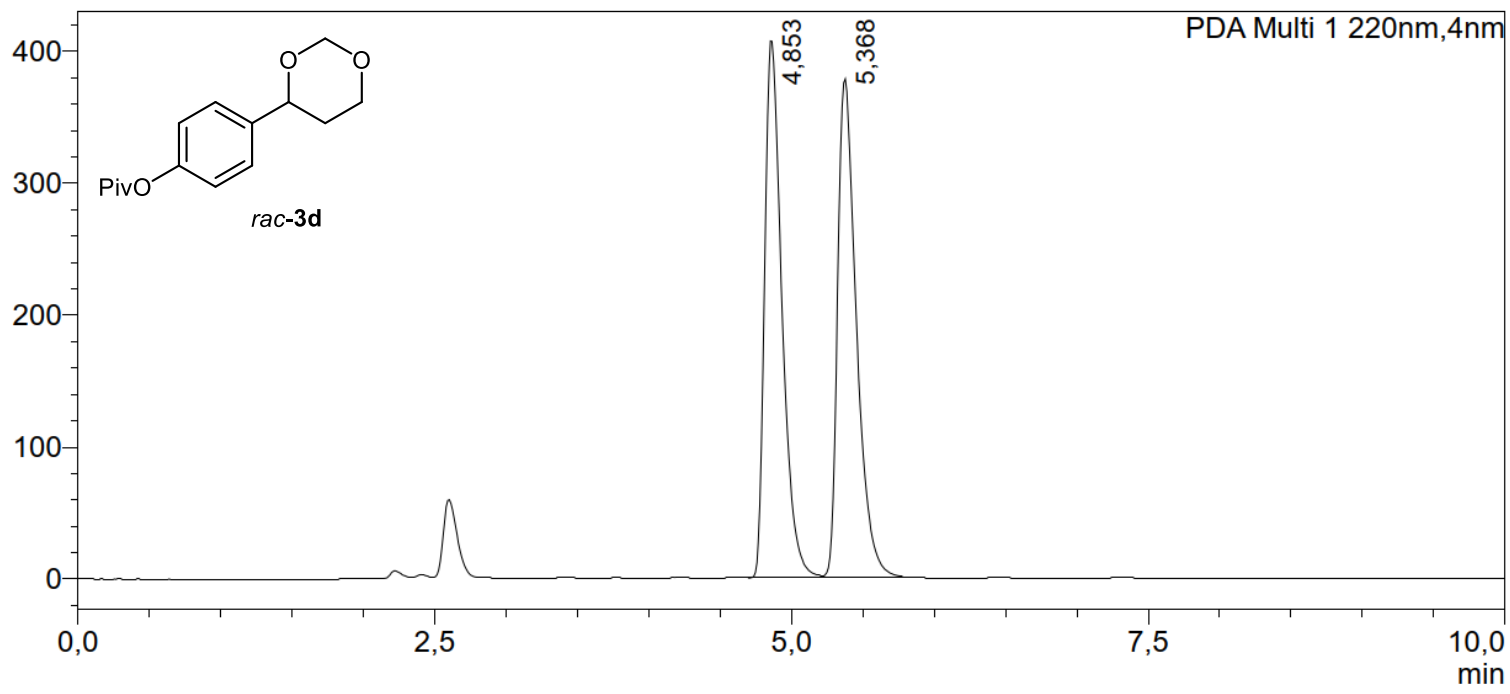

\begin{tabular}{|r|r|r|}
\hline Peak\# & Ret. Time & \multicolumn{1}{c|}{ Area\% } \\
\hline 1 & 4,853 & 49,709 \\
\hline 2 & 5,368 & 50,291 \\
\hline Total & & 100,000 \\
\hline
\end{tabular}

mAU

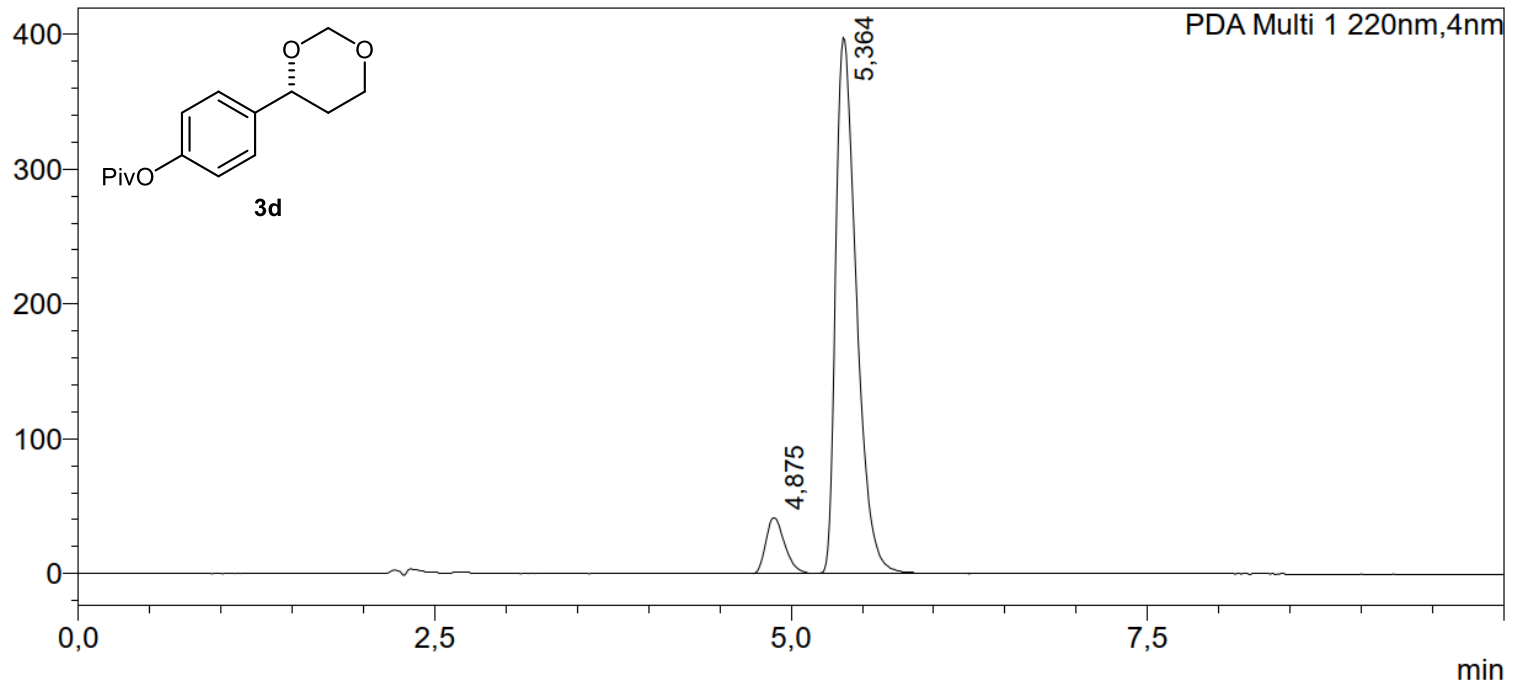

\begin{tabular}{|r|r|r|}
\hline Peak\# & Ret. Time & \multicolumn{1}{|c|}{ Area\% } \\
\hline 1 & 4,875 & 8,803 \\
\hline 2 & 5,364 & 91,197 \\
\hline Total & & 100,000 \\
\hline
\end{tabular}


The Catalytic Asymmetric Intermolecular Prins Reaction Copies of HPLC traces

(Chiralcel OJ-3 column, Heptane/i-PrOH 95:5, 1 mL/min, $25^{\circ} \mathrm{C}, 220 \mathrm{~nm}$ )

mAU

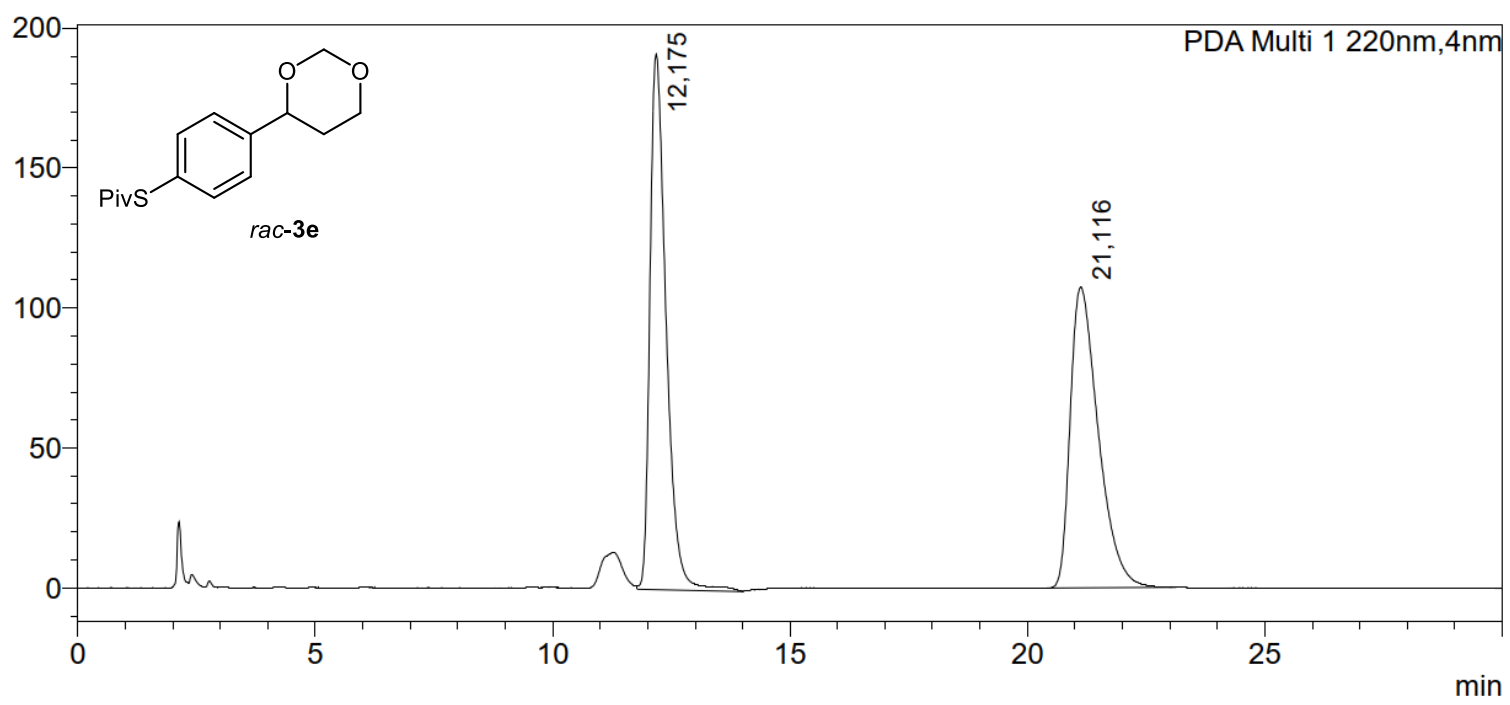

\begin{tabular}{|r|r|r|}
\hline Peak\# & Ret. Time & \multicolumn{1}{c|}{ Area\% } \\
\hline 1 & 12,175 & 50,650 \\
\hline 2 & 21,116 & 49,350 \\
\hline Total & & 100,000 \\
\hline
\end{tabular}

mAU

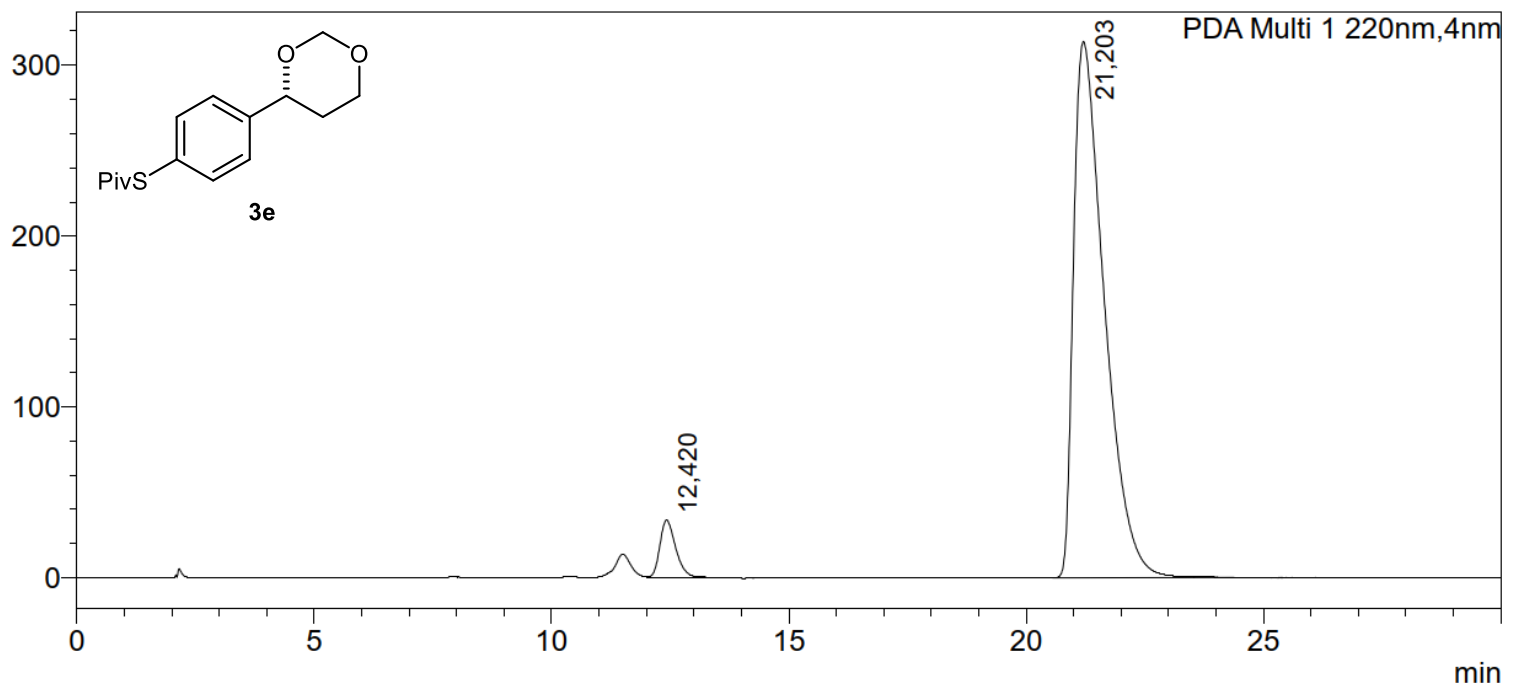

\begin{tabular}{|r|r|r|}
\hline Peak\# & Ret. Time & \multicolumn{1}{c|}{ Area\% } \\
\hline 1 & 12,420 & 5,193 \\
\hline 2 & 21,203 & 94,807 \\
\hline Total & & 100,000 \\
\hline
\end{tabular}


The Catalytic Asymmetric Intermolecular Prins Reaction Copies of HPLC traces

(Chiralpak IC-3 column, Heptane/i-PrOH 98:2, 1 mL/min, $25^{\circ} \mathrm{C}, 220 \mathrm{~nm}$ )

mAU

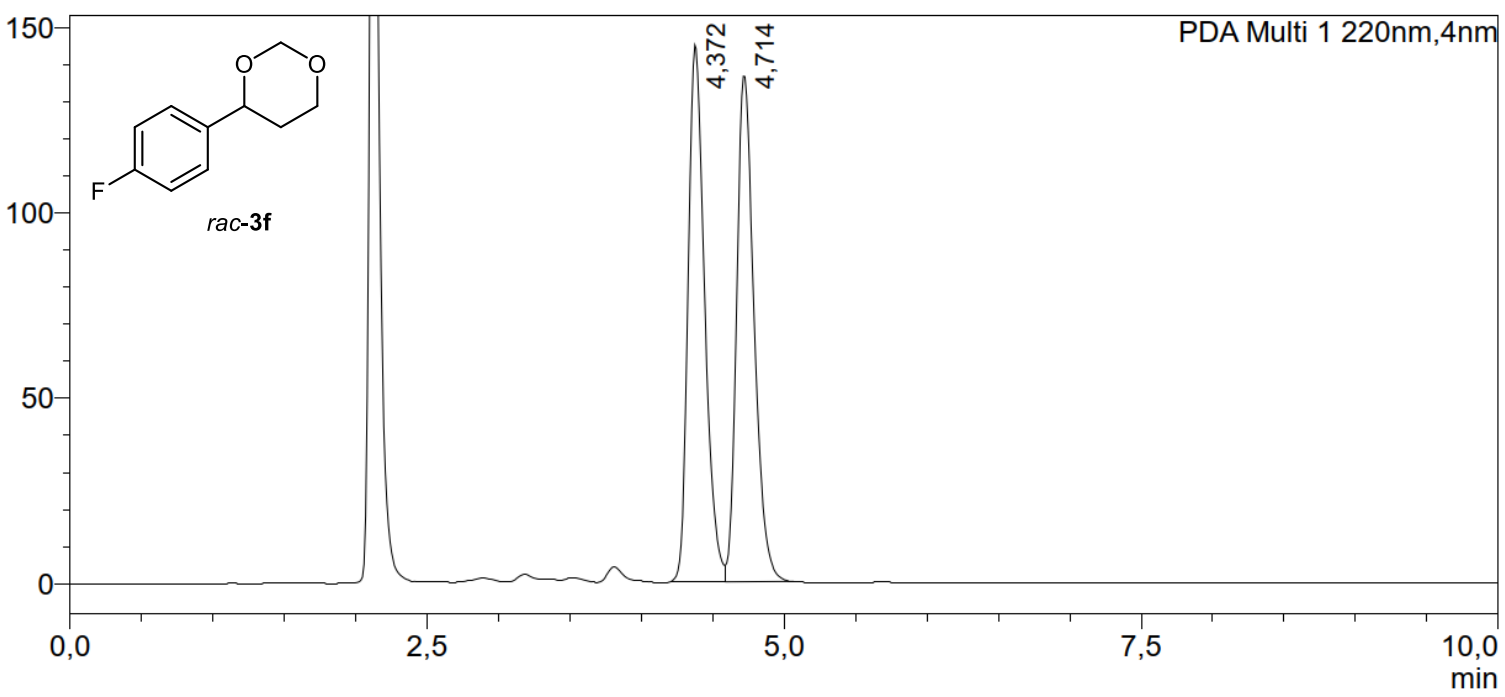

\begin{tabular}{|r|r|r|}
\hline Peak\# & Ret. Time & \multicolumn{1}{c|}{ Area\% } \\
\hline 1 & 4,372 & 50,187 \\
\hline 2 & 4,714 & 49,813 \\
\hline Total & & 100,000 \\
\hline
\end{tabular}

$\mathrm{mAU}$

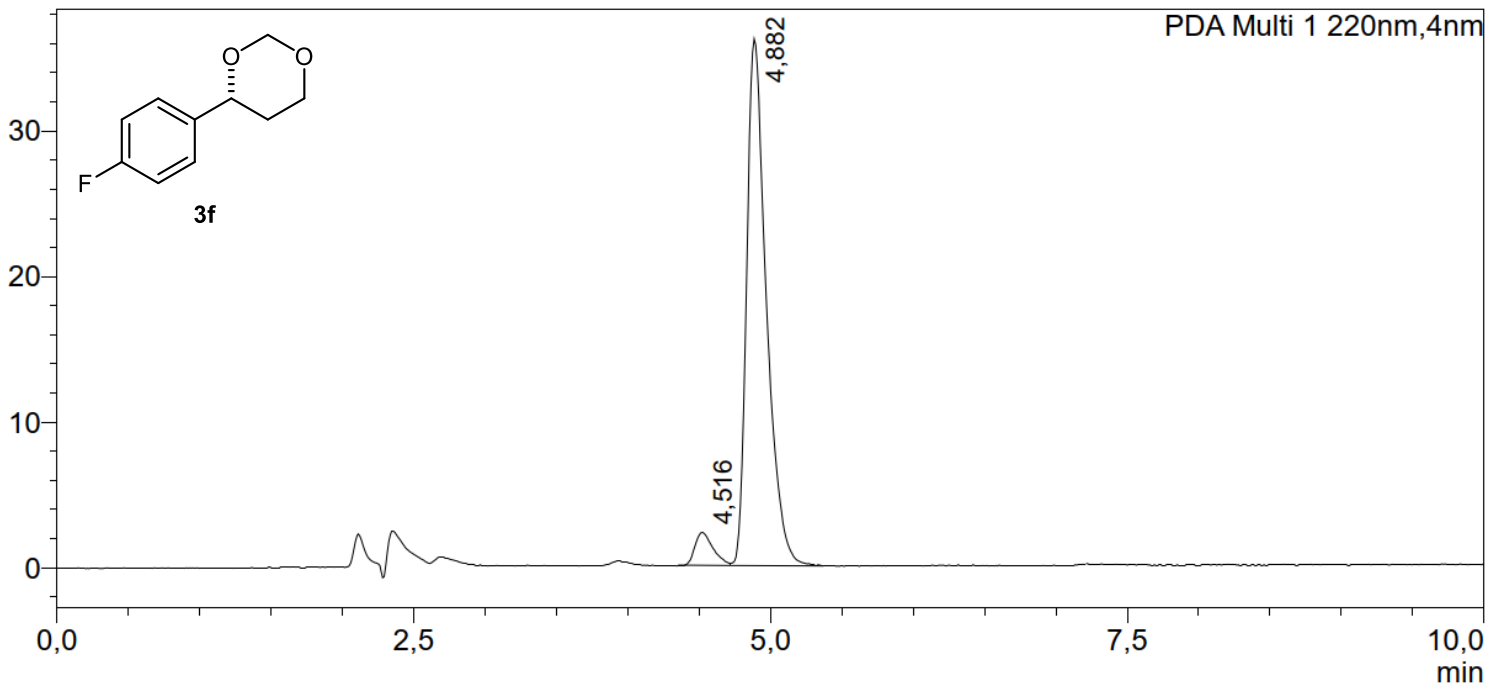

\begin{tabular}{|r|r|r|}
\hline Peak\# & Ret. Time & \multicolumn{1}{|c|}{ Area\% } \\
\hline 1 & 4,516 & 5,441 \\
\hline 2 & 4,882 & 94,559 \\
\hline Total & & 100,000 \\
\hline
\end{tabular}


The Catalytic Asymmetric Intermolecular Prins Reaction Copies of HPLC traces

(Chiralpak IC-3 column, Heptane/i-PrOH 97:3, $0.5 \mathrm{~mL} / \mathrm{min}, 25^{\circ} \mathrm{C}, 220 \mathrm{~nm}$ )

mAU

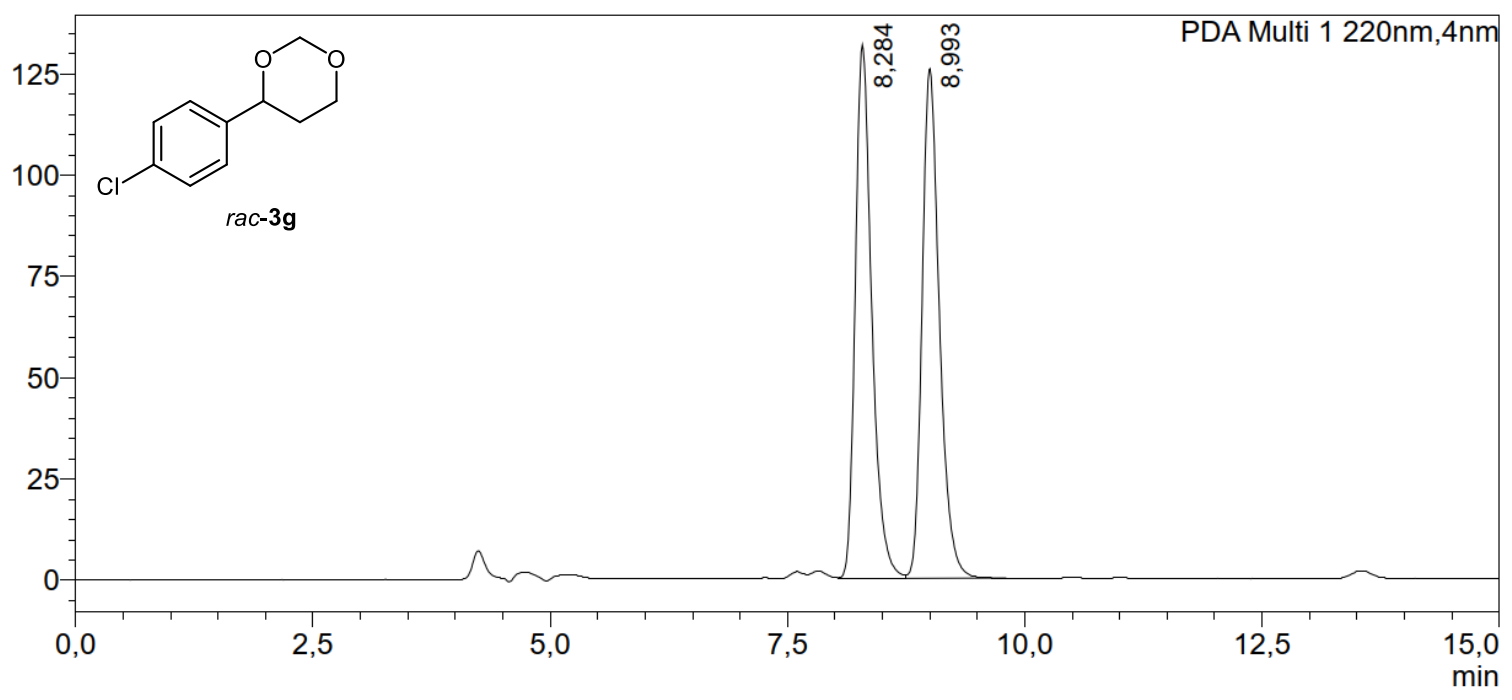

\begin{tabular}{|r|r|r|}
\hline Peak\# & Ret. Time & \multicolumn{1}{c|}{ Area\% } \\
\hline 1 & 8,284 & 49,852 \\
\hline 2 & 8,993 & 50,148 \\
\hline Total & & 100,000 \\
\hline
\end{tabular}

mAU

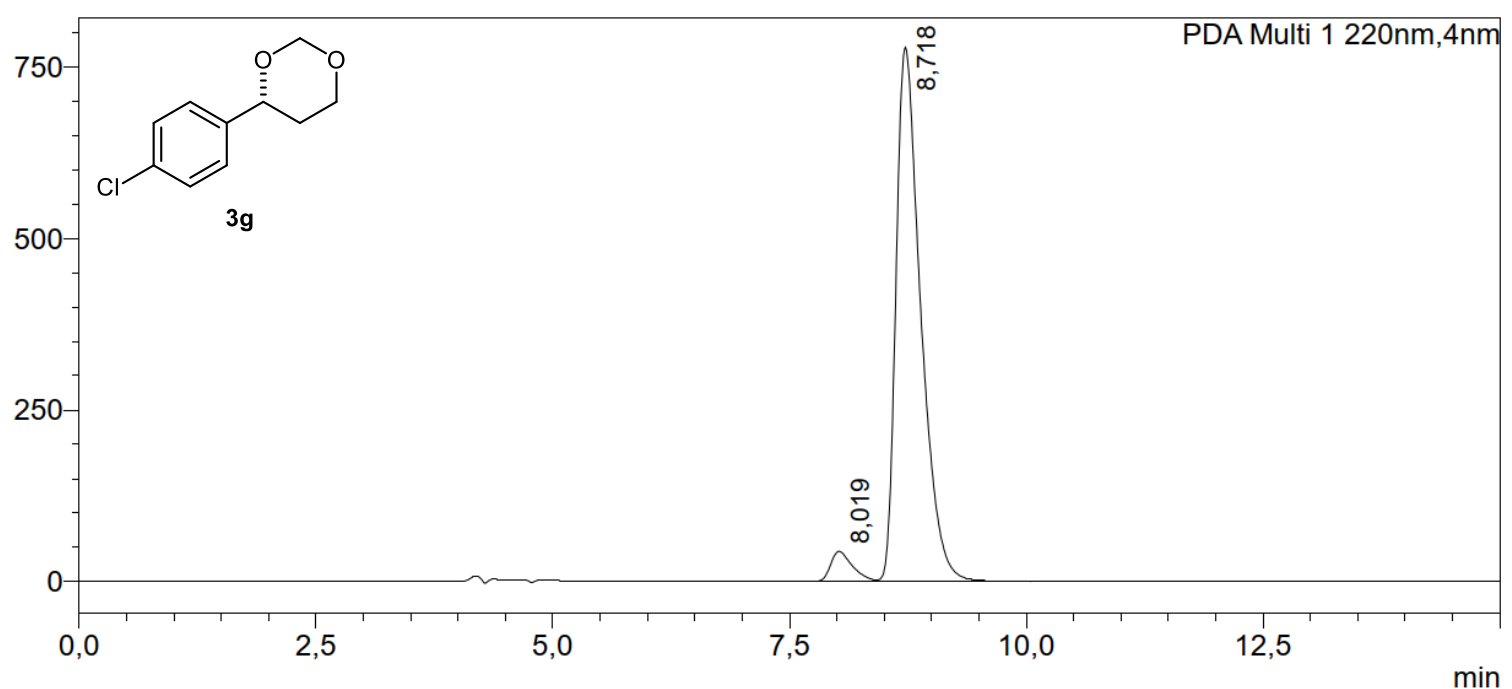

\begin{tabular}{|r|r|r|}
\hline Peak\# & Ret. Time & \multicolumn{1}{c|}{ Area\% } \\
\hline 1 & 8,019 & 4,765 \\
\hline 2 & 8,718 & 95,235 \\
\hline Total & & 100,000 \\
\hline
\end{tabular}


The Catalytic Asymmetric Intermolecular Prins Reaction Copies of HPLC traces

(Chiralpak IC-3 column, Heptane/i-PrOH 97:3, $0.5 \mathrm{~mL} / \mathrm{min}, 25^{\circ} \mathrm{C}, 220 \mathrm{~nm}$ )

mAU

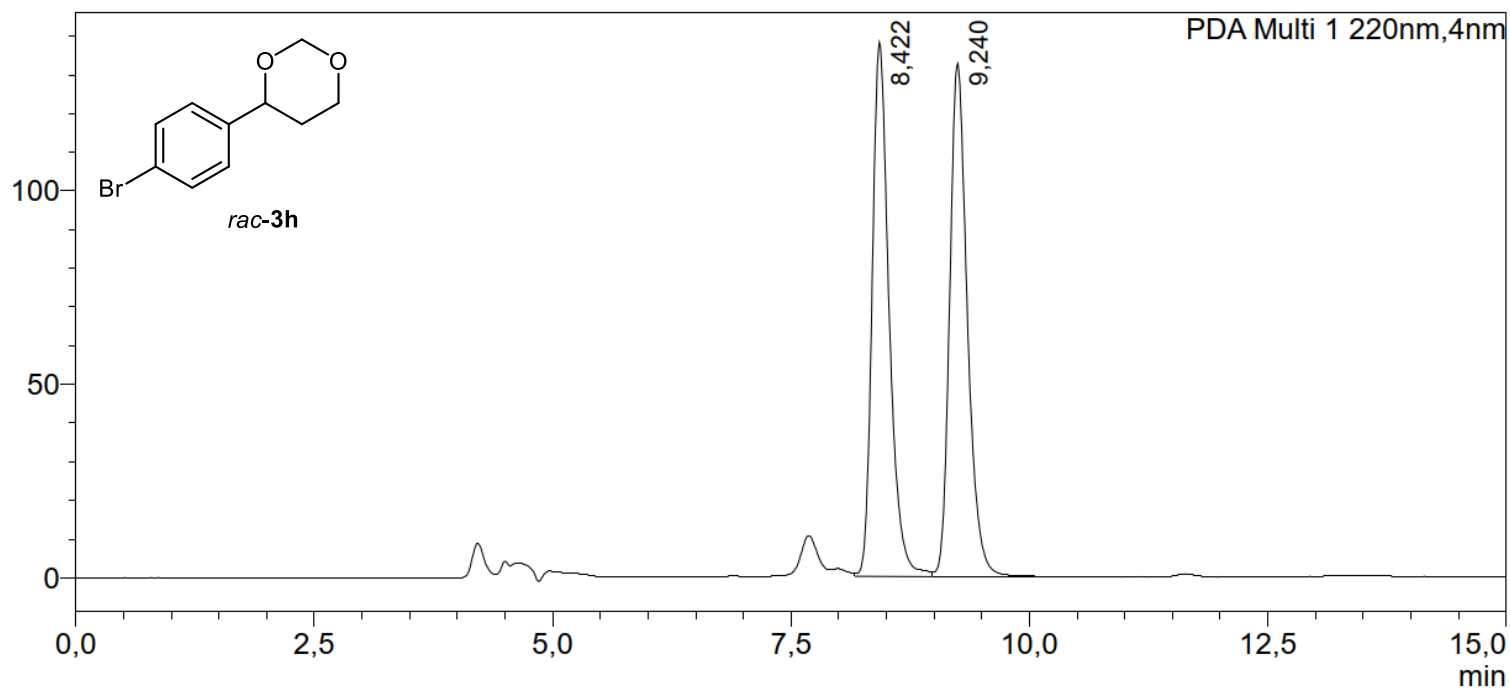

\begin{tabular}{|r|r|r|}
\hline Peak\# & Ret. Time & \multicolumn{1}{c|}{ Area\% } \\
\hline 1 & 8,422 & 49,904 \\
\hline 2 & 9,240 & 50,096 \\
\hline Total & & 100,000 \\
\hline
\end{tabular}

mAU

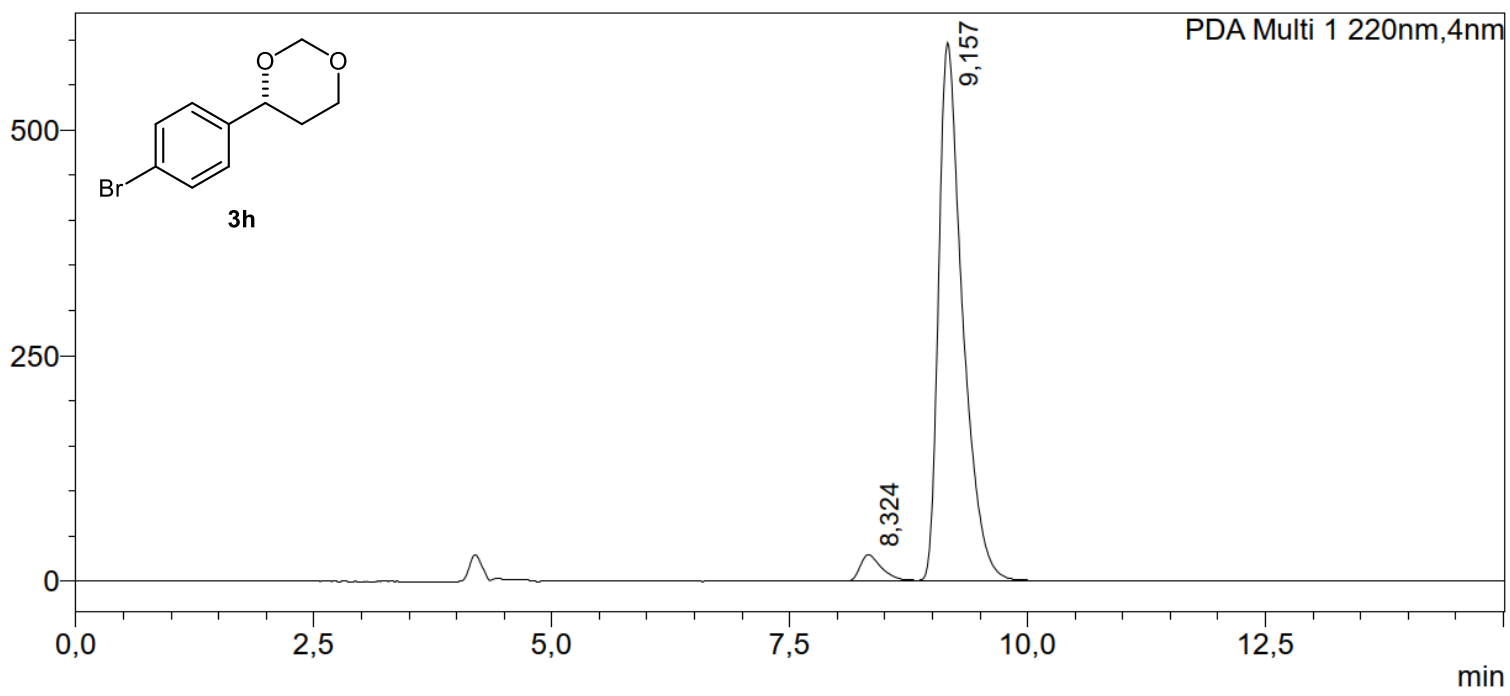

\begin{tabular}{|r|r|r|}
\hline Peak\# & Ret. Time & \multicolumn{1}{c|}{ Area\% } \\
\hline 1 & 8,324 & 4,399 \\
\hline 2 & 9,157 & 95,601 \\
\hline Total & & 100,000 \\
\hline
\end{tabular}


The Catalytic Asymmetric Intermolecular Prins Reaction Copies of HPLC traces

(Chiralcel OJ-3 column, Heptane/i-PrOH 95:5, $1 \mathrm{~mL} / \mathrm{min}, 25^{\circ} \mathrm{C}, 220 \mathrm{~nm}$ )

mAU

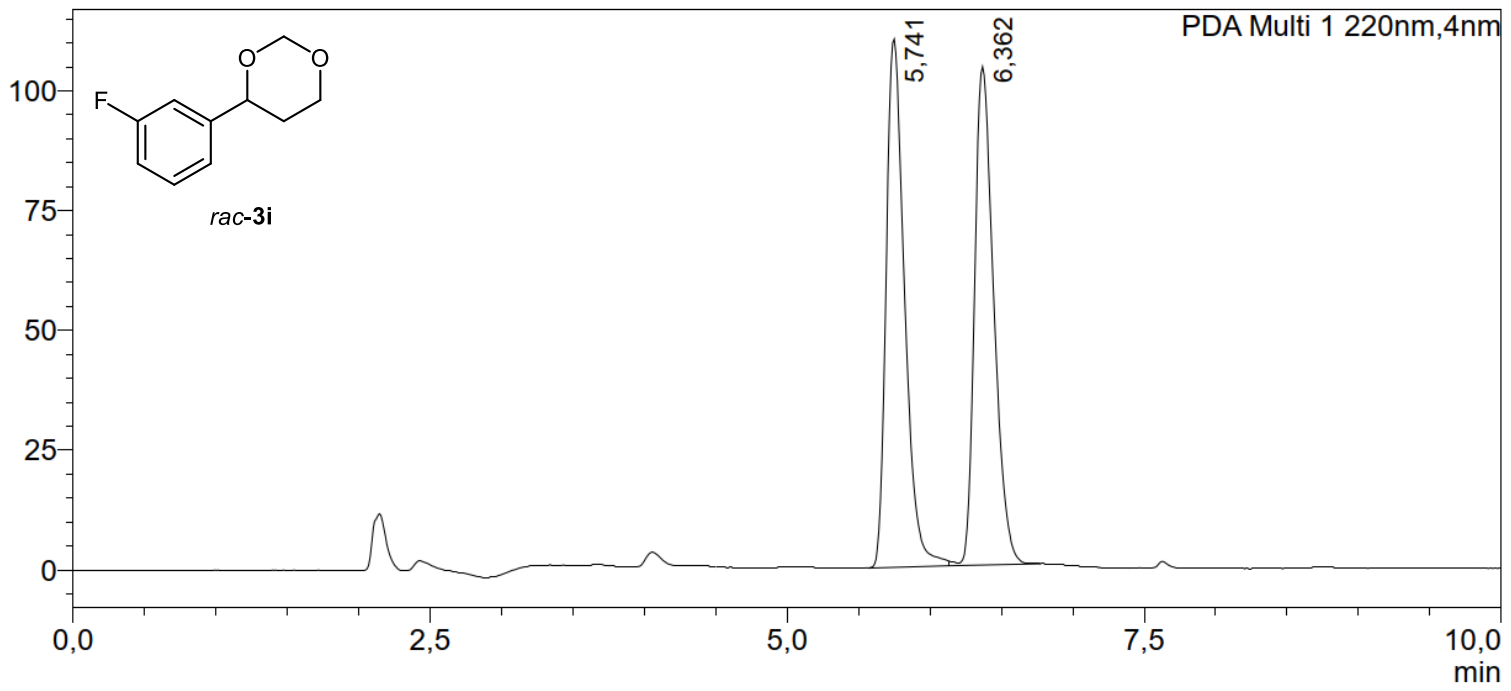

\begin{tabular}{|r|r|r|}
\hline Peak\# & Ret. Time & \multicolumn{1}{c|}{ Area\% } \\
\hline 1 & 5,741 & 50,513 \\
\hline 2 & 6,362 & 49,487 \\
\hline Total & & 100,000 \\
\hline
\end{tabular}

$\mathrm{mAU}$

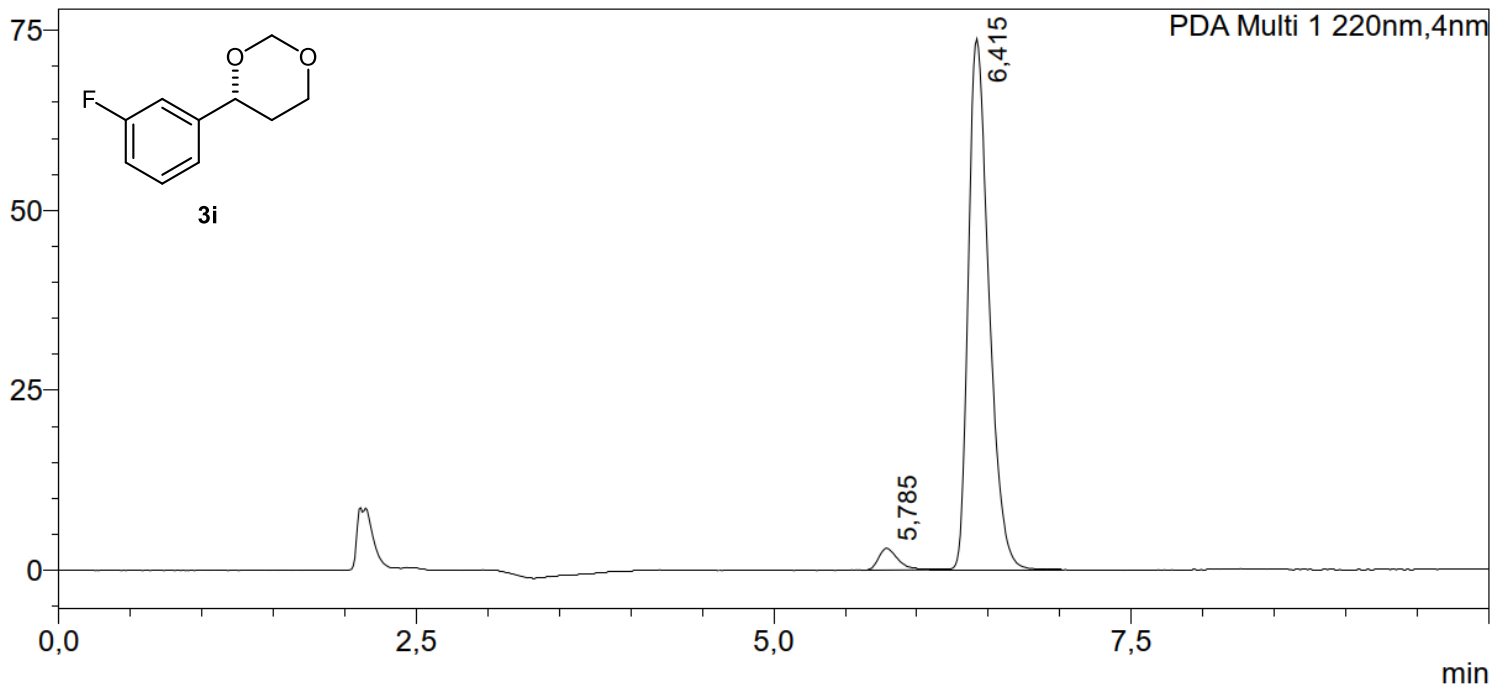

\begin{tabular}{|r|r|r|}
\hline Peak\# & Ret. Time & \multicolumn{1}{|c|}{ Area\% } \\
\hline 1 & 5,785 & 3,741 \\
\hline 2 & 6,415 & 96,259 \\
\hline Total & & 100,000 \\
\hline
\end{tabular}


The Catalytic Asymmetric Intermolecular Prins Reaction Copies of HPLC traces

(Chiralpak IC-3 column, Heptane/i-PrOH 97:3, $0.5 \mathrm{~mL} / \mathrm{min}, 25^{\circ} \mathrm{C}, 220 \mathrm{~nm}$ )

mAU

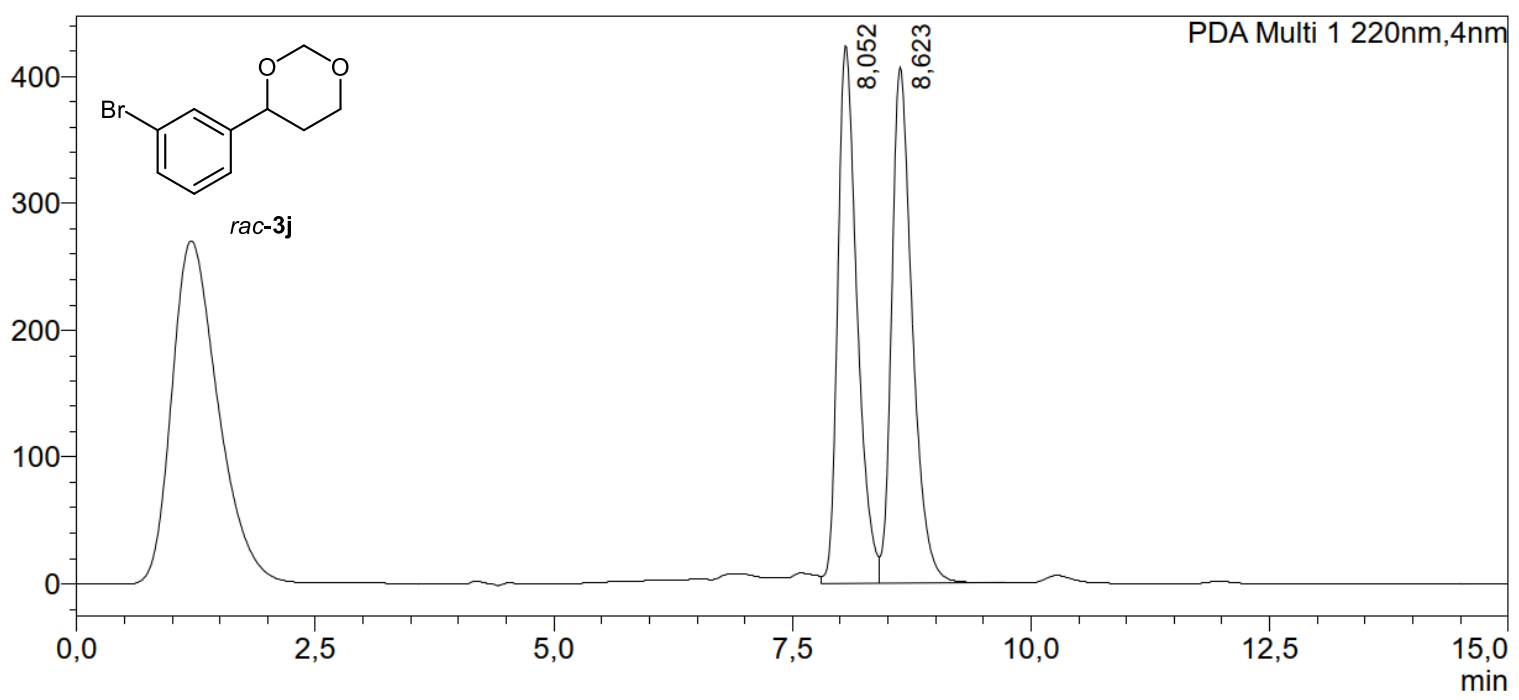

\begin{tabular}{|r|r|r|}
\hline Peak\# & Ret. Time & \multicolumn{1}{c|}{ Area\% } \\
\hline 1 & 8,052 & 49,431 \\
\hline 2 & 8,623 & 50,569 \\
\hline Total & & 100,000 \\
\hline
\end{tabular}

$\mathrm{mAU}$

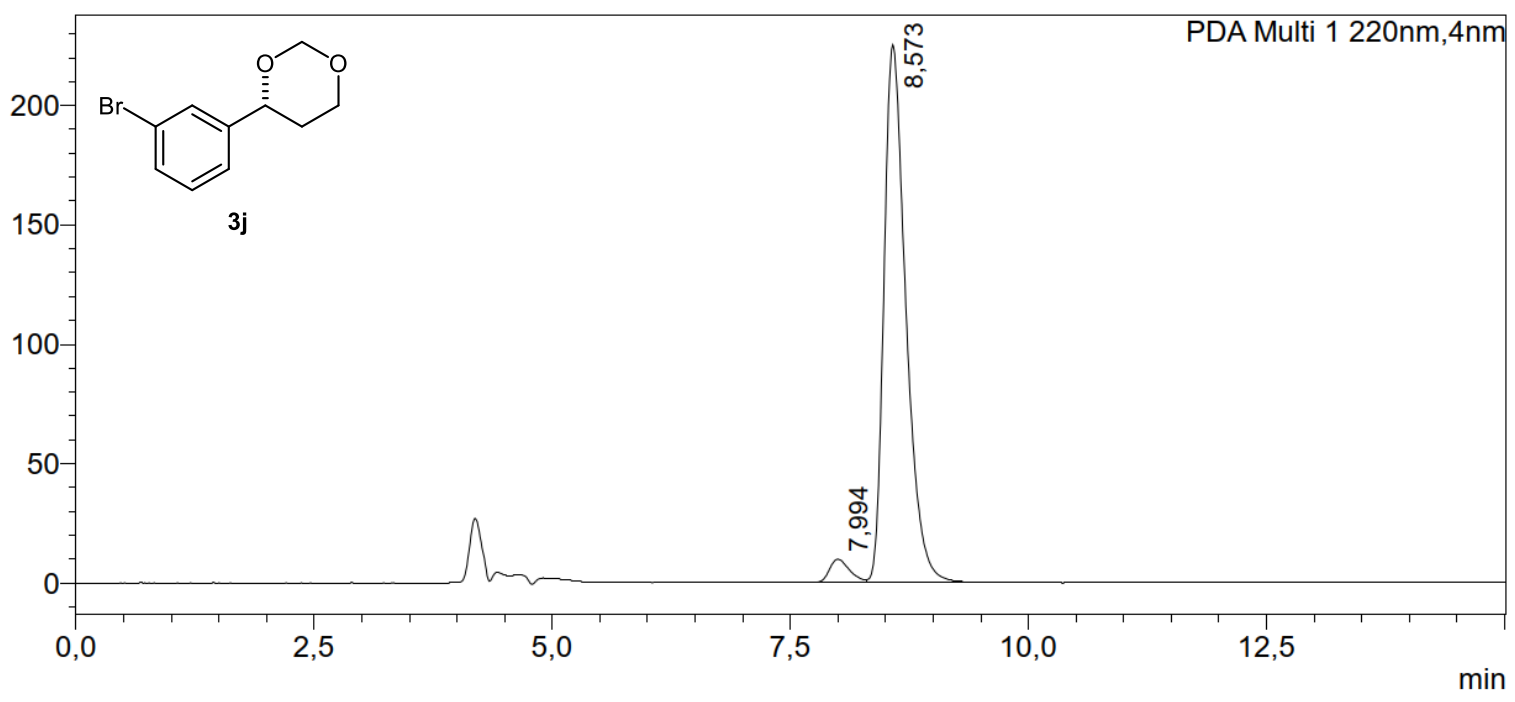

\begin{tabular}{|r|r|r|}
\hline Peak\# & Ret. Time & \multicolumn{1}{|c|}{ Area\% } \\
\hline 1 & 7,994 & 3,945 \\
\hline 2 & 8,573 & 96,055 \\
\hline Total & & 100,000 \\
\hline
\end{tabular}


The Catalytic Asymmetric Intermolecular Prins Reaction Copies of HPLC traces

(Chiralpak IB-3 column, Heptane/i-PrOH 95:5, 1 mL/min, $25^{\circ} \mathrm{C}, 220 \mathrm{~nm}$ )

mAU

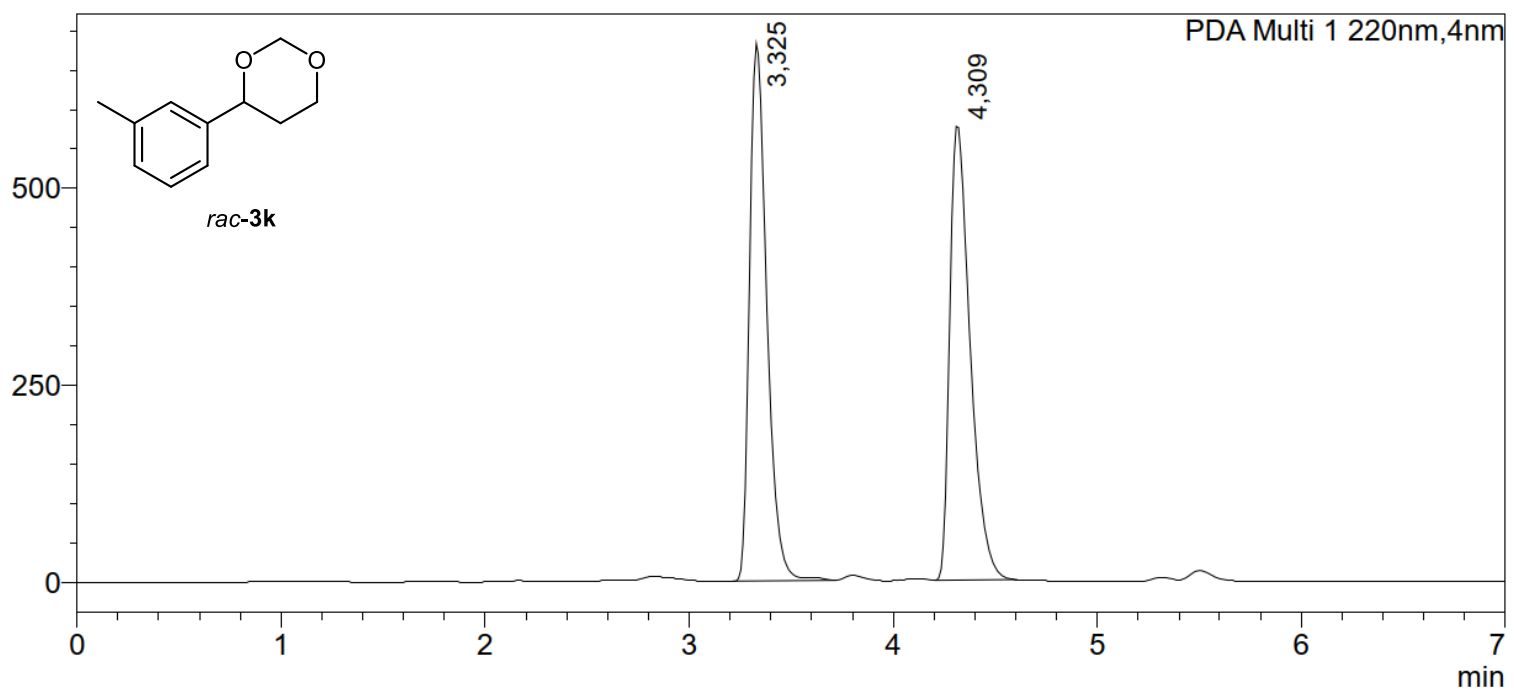

\begin{tabular}{|r|r|r|}
\hline Peak\# & Ret. Time & Area\% \\
\hline 1 & 3,325 & 49,867 \\
\hline 2 & 4,309 & 50,133 \\
\hline Total & & 100,000 \\
\hline
\end{tabular}

mAU

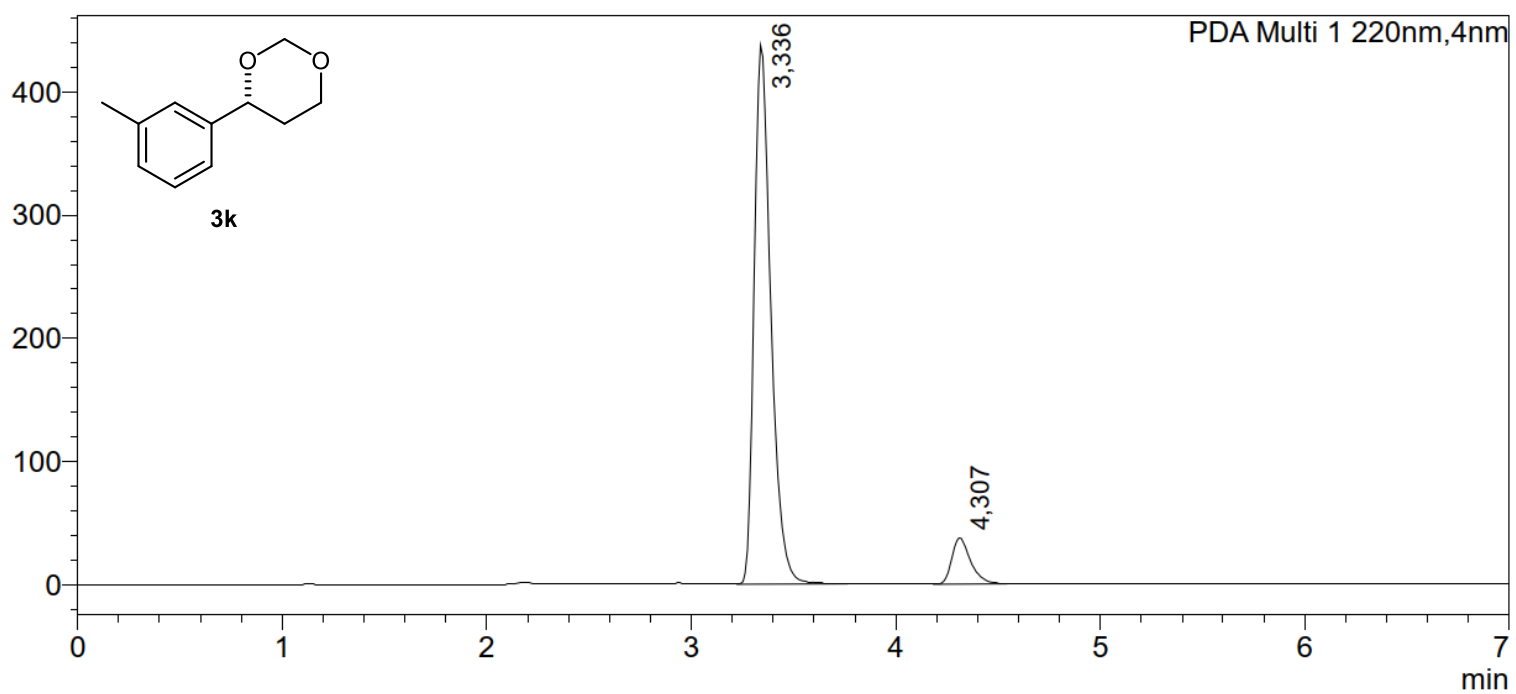

\begin{tabular}{|r|r|r|}
\hline Peak\# & Ret. Time & \multicolumn{1}{c|}{ Area\% } \\
\hline 1 & 3,336 & 93,151 \\
\hline 2 & 4,307 & 6,849 \\
\hline Total & & 100,000 \\
\hline
\end{tabular}


The Catalytic Asymmetric Intermolecular Prins Reaction Copies of HPLC traces

(Chiralpak IB-3 column, Heptane/i-PrOH 95:5, 1 mL/min, $25^{\circ} \mathrm{C}, 220 \mathrm{~nm}$ )

mAU

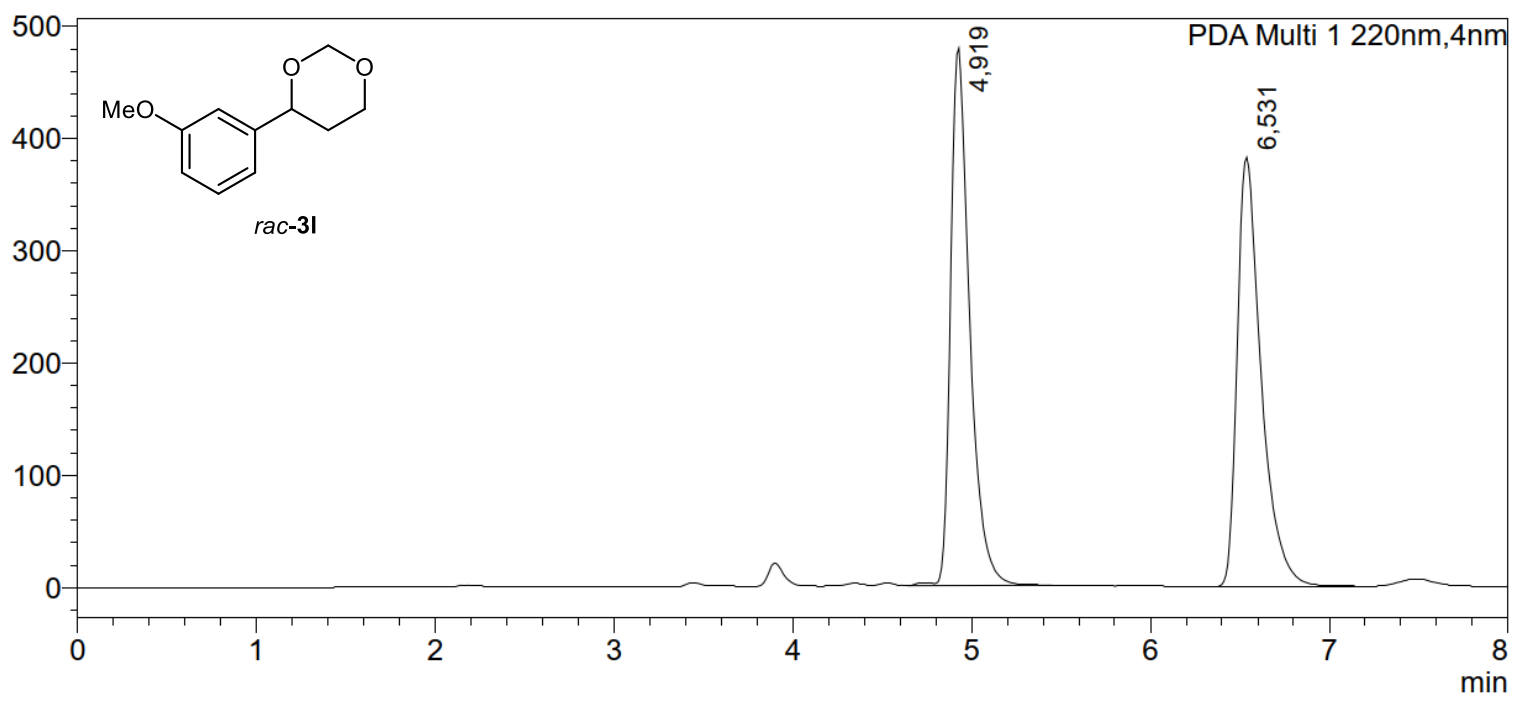

\begin{tabular}{|r|r|r|}
\hline Peak\# & Ret. Time & \multicolumn{1}{|c|}{ Area\% } \\
\hline 1 & 4,919 & 50,028 \\
\hline 2 & 6,531 & 49,972 \\
\hline Total & & 100,000 \\
\hline
\end{tabular}

mAU

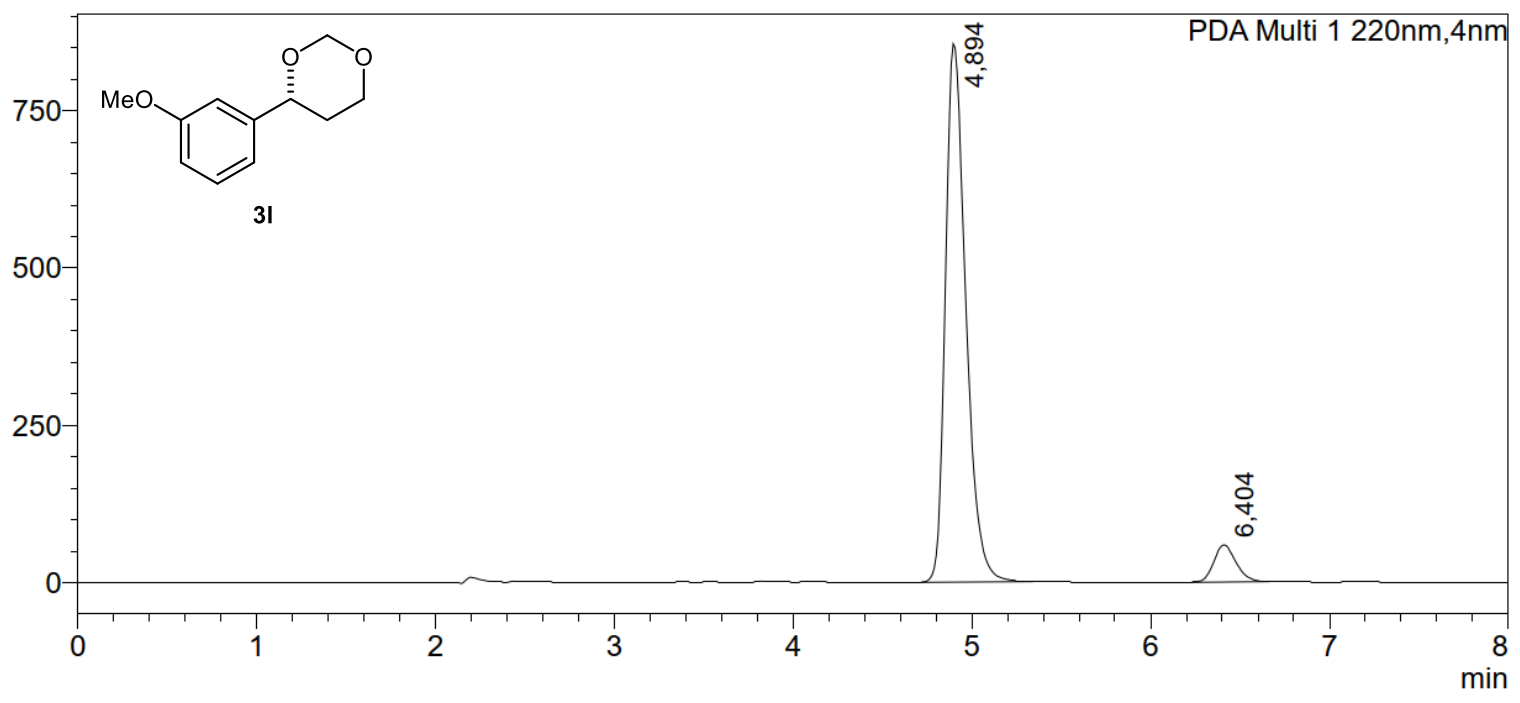

\begin{tabular}{|r|r|r|}
\hline Peak\# & Ret. Time & \multicolumn{1}{|c|}{ Area\% } \\
\hline 1 & 4,894 & 93,044 \\
\hline 2 & 6,404 & 6,956 \\
\hline Total & & 100,000 \\
\hline
\end{tabular}


The Catalytic Asymmetric Intermolecular Prins Reaction Copies of HPLC traces

(Chiralcel OJ-3 column, Heptane/i-PrOH 95:5, 1 mL/min, $25^{\circ} \mathrm{C}, 220 \mathrm{~nm}$ )

$\mathrm{mAU}$

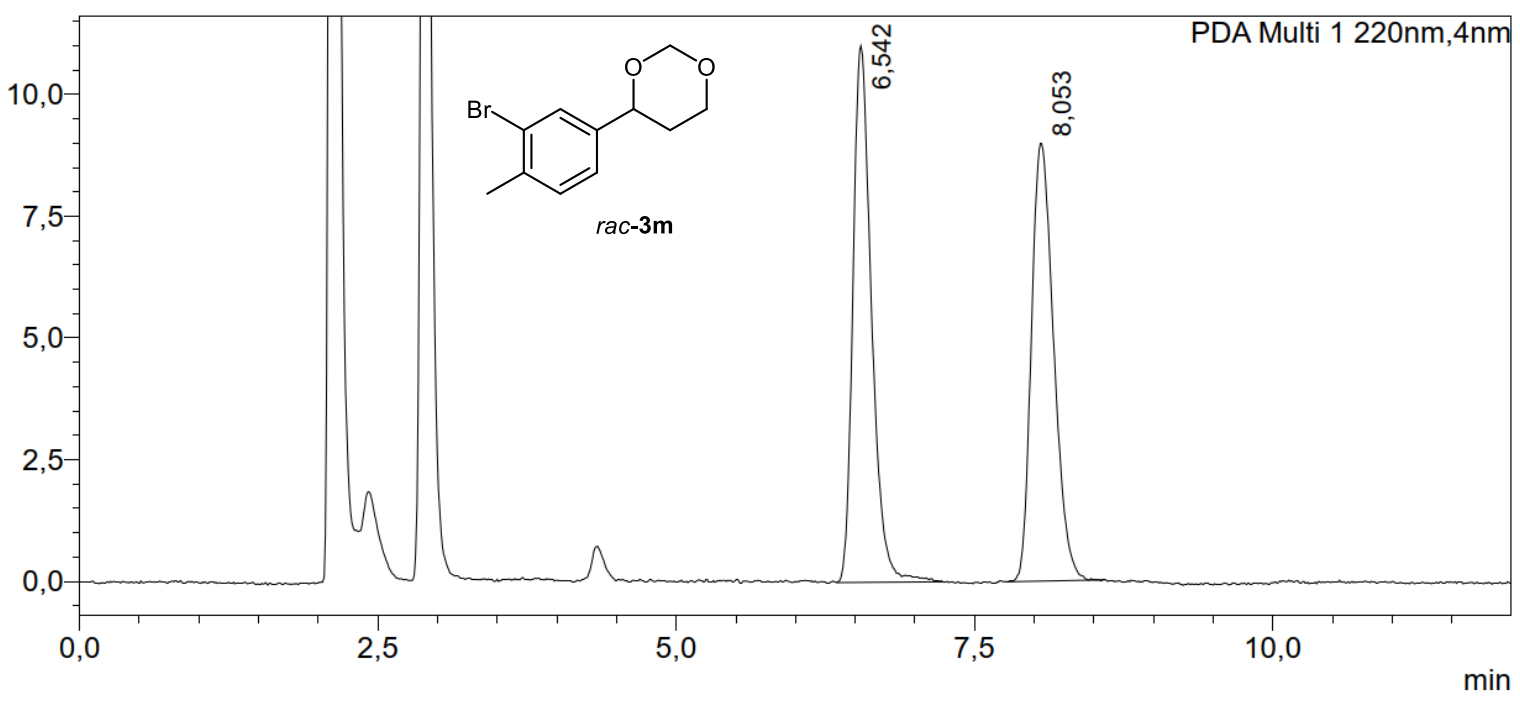

\begin{tabular}{|r|r|r|}
\hline Peak\# & Ret. Time & \multicolumn{1}{c|}{ Area\% } \\
\hline 1 & 6,542 & 50,512 \\
\hline 2 & 8,053 & 49,488 \\
\hline Total & & 100,000 \\
\hline
\end{tabular}

mAU

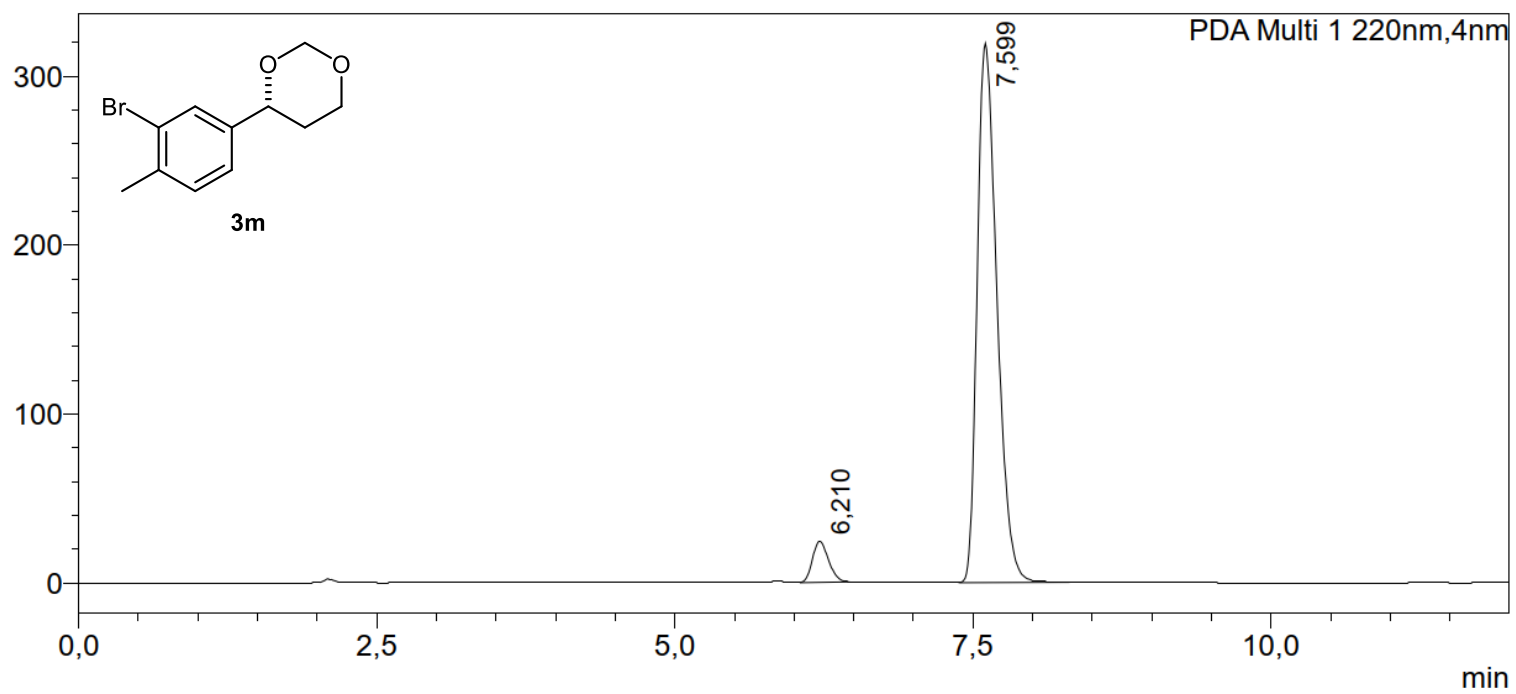

\begin{tabular}{|r|r|r|}
\hline Peak\# & Ret. Time & \multicolumn{1}{|c|}{ Area\% } \\
\hline 1 & 6,210 & 5,849 \\
\hline 2 & 7,599 & 94,151 \\
\hline Total & & 100,000 \\
\hline
\end{tabular}


The Catalytic Asymmetric Intermolecular Prins Reaction Copies of HPLC traces

(Chiralpak IB-3 column, Heptane/i-PrOH 80:20, $1 \mathrm{~mL} / \mathrm{min}, 25^{\circ} \mathrm{C}, 274 \mathrm{~nm}$ )

mAU

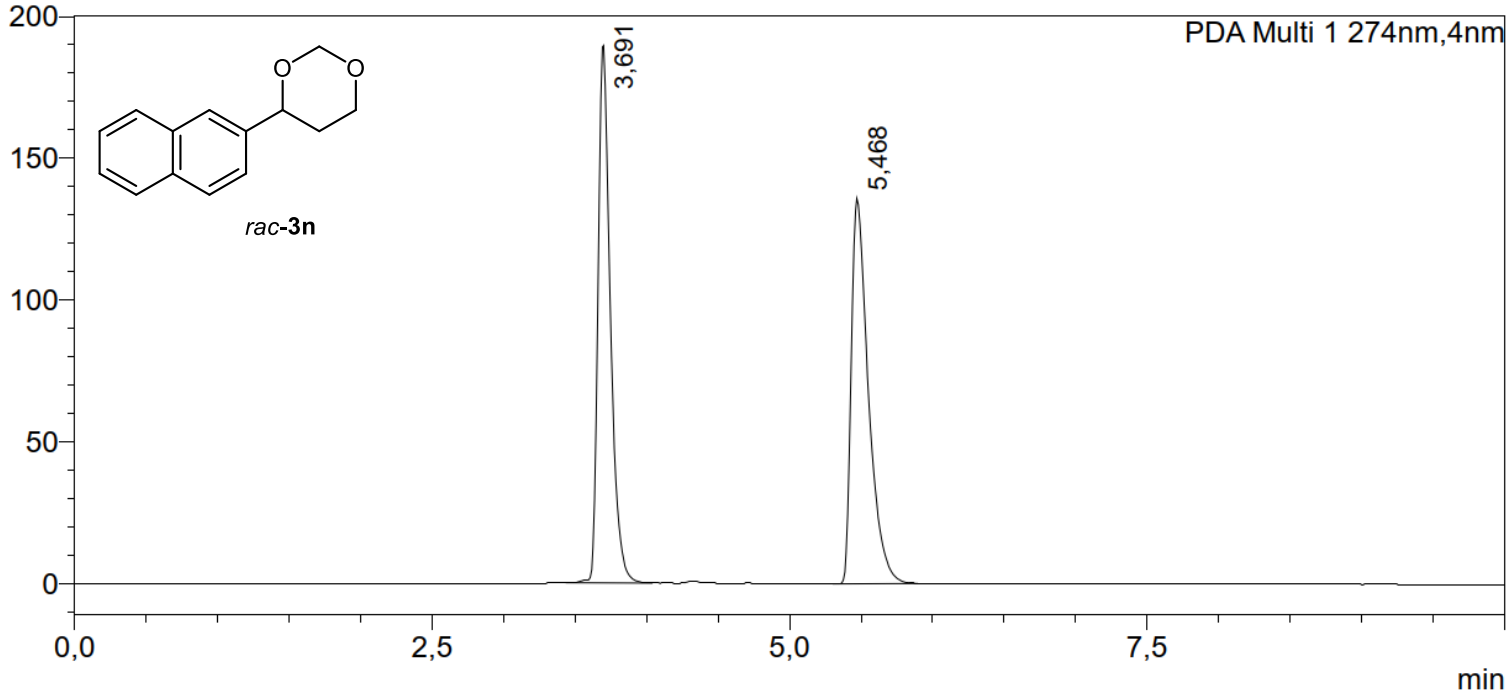

\begin{tabular}{|r|r|r|}
\hline Peak\# & Ret. Time & \multicolumn{1}{|c|}{ Area\% } \\
\hline 1 & 3,691 & 50,072 \\
\hline 2 & 5,468 & 49,928 \\
\hline Total & & 100,000 \\
\hline
\end{tabular}

mAU

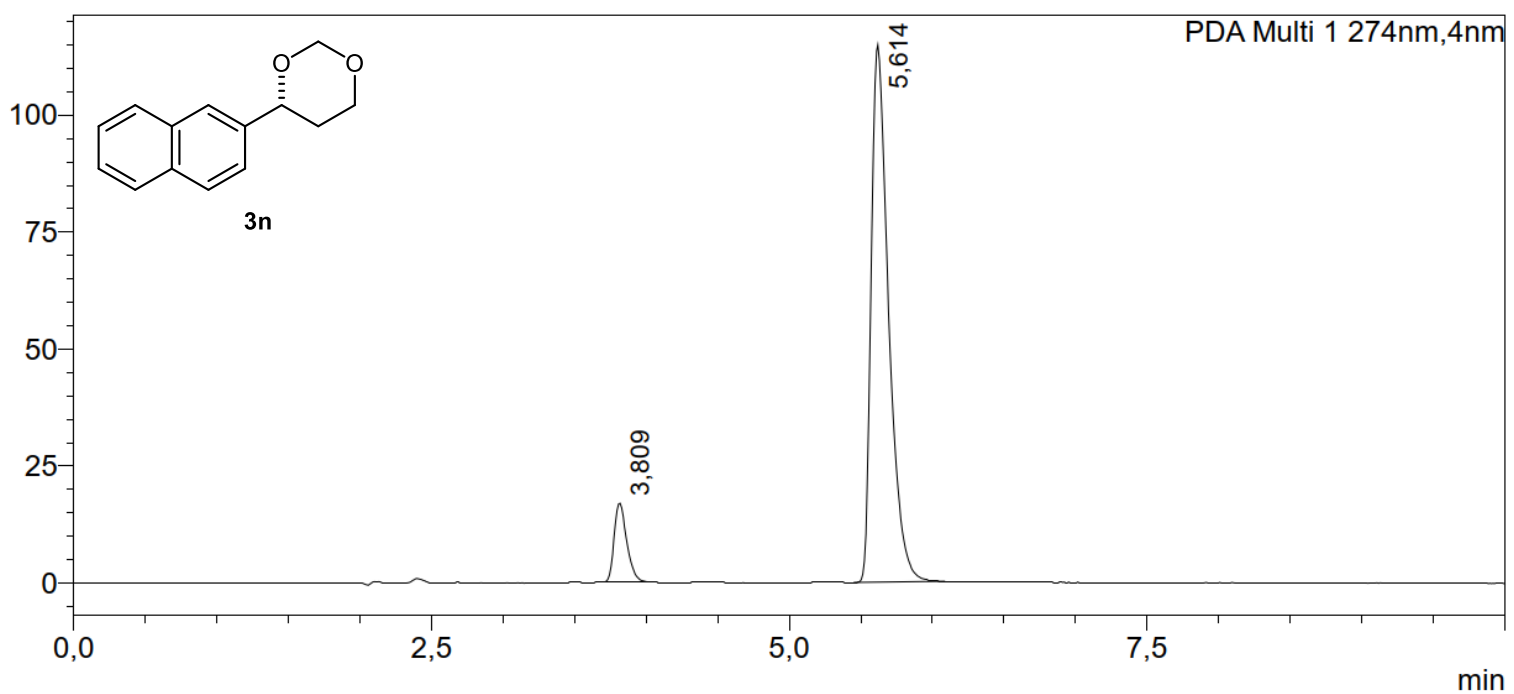

\begin{tabular}{|r|r|r|}
\hline Peak\# & Ret. Time & \multicolumn{1}{|c|}{ Area\% } \\
\hline 1 & 3,809 & 9,601 \\
\hline 2 & 5,614 & 90,399 \\
\hline Total & & 100,000 \\
\hline
\end{tabular}


The Catalytic Asymmetric Intermolecular Prins Reaction Copies of HPLC traces

(Chiralcel OJ-3 column, Heptane/i-PrOH 95:5, 1 mL/min, $25^{\circ} \mathrm{C}, 220 \mathrm{~nm}$ )

mAU

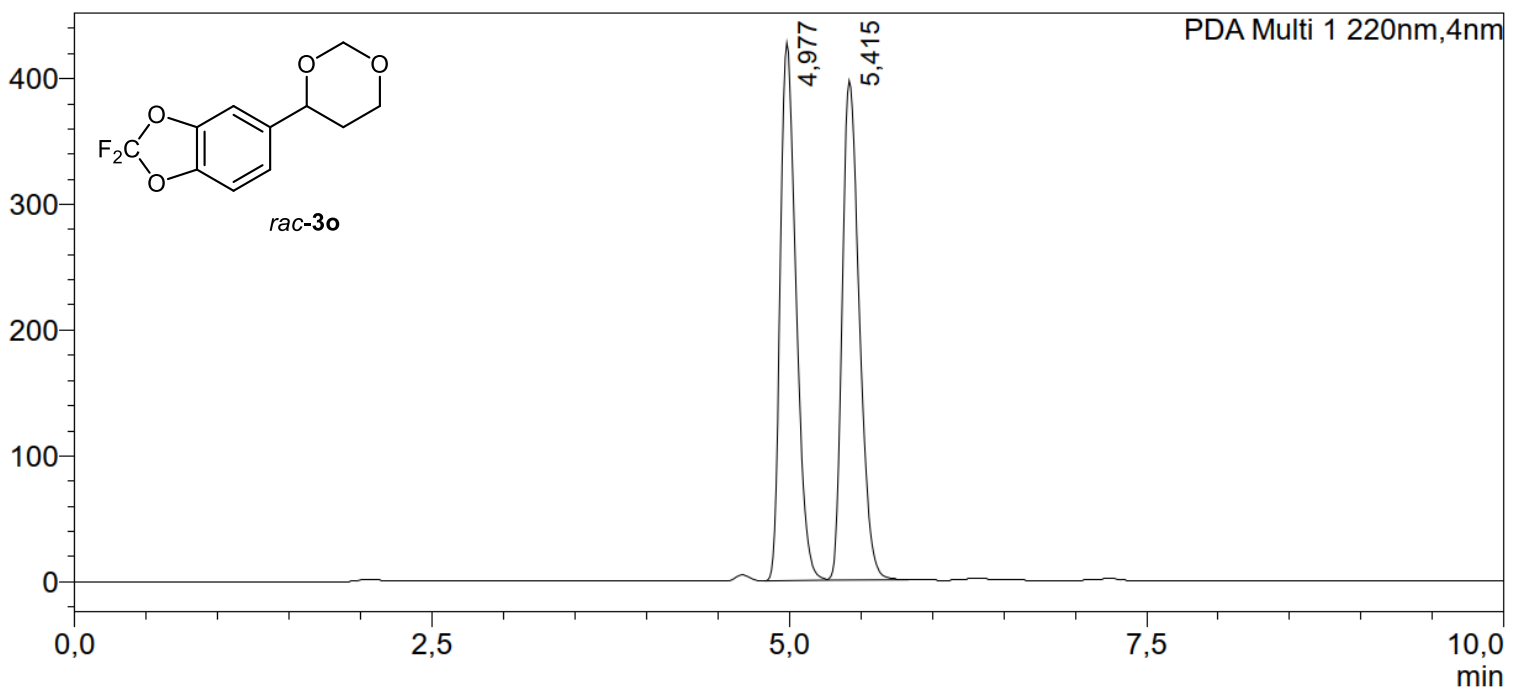

\begin{tabular}{|r|r|r|}
\hline Peak\# & Ret. Time & \multicolumn{1}{c|}{ Area\% } \\
\hline 1 & 4,977 & 49,855 \\
\hline 2 & 5,415 & 50,145 \\
\hline Total & & 100,000 \\
\hline
\end{tabular}

$\mathrm{mAU}$

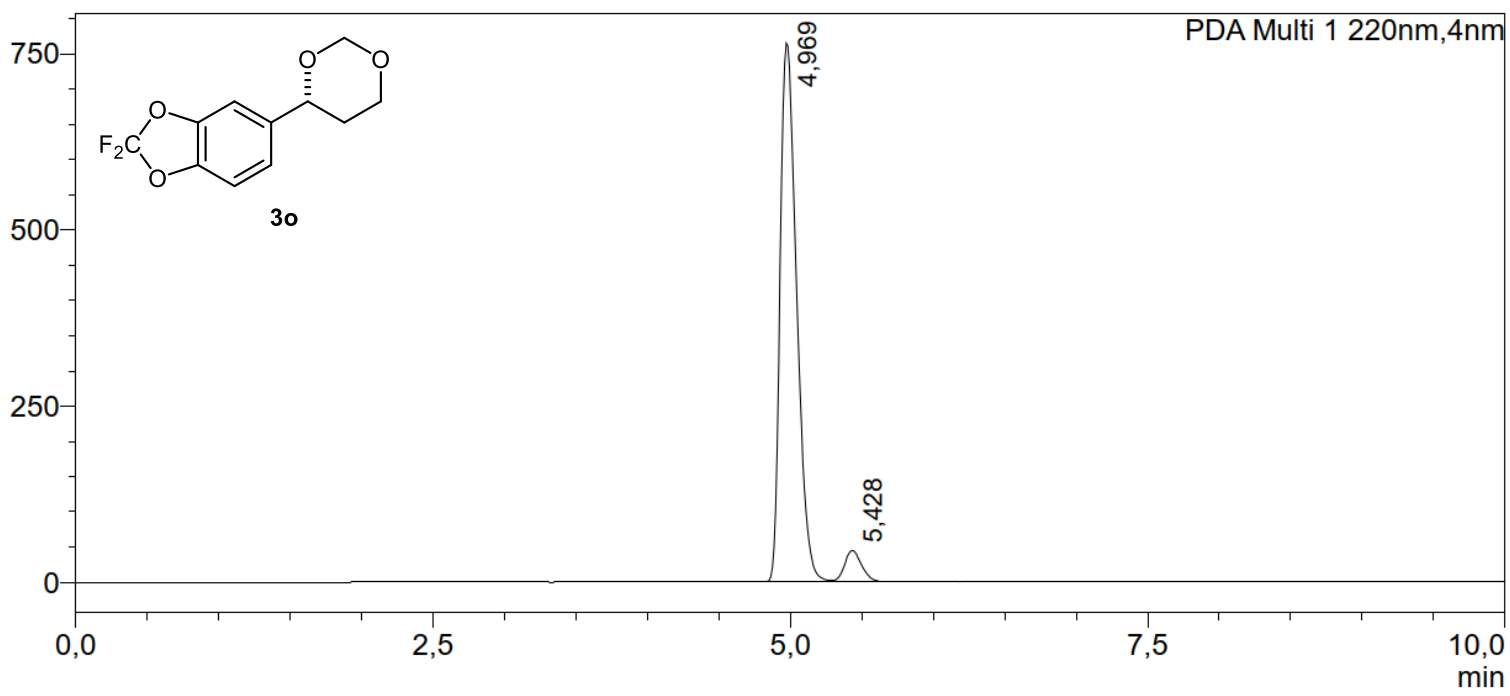

\begin{tabular}{|r|r|r|}
\hline Peak\# & Ret. Time & \multicolumn{1}{c|}{ Area\% } \\
\hline 1 & 4,969 & 94,335 \\
\hline 2 & 5,428 & 5,665 \\
\hline Total & & 100,000 \\
\hline
\end{tabular}


The Catalytic Asymmetric Intermolecular Prins Reaction Copies of HPLC traces

(Chiralpak IC-3 column, Heptane/i-PrOH 97:3, $0.5 \mathrm{~mL} / \mathrm{min}, 25^{\circ} \mathrm{C}, 220 \mathrm{~nm}$ )

mAU

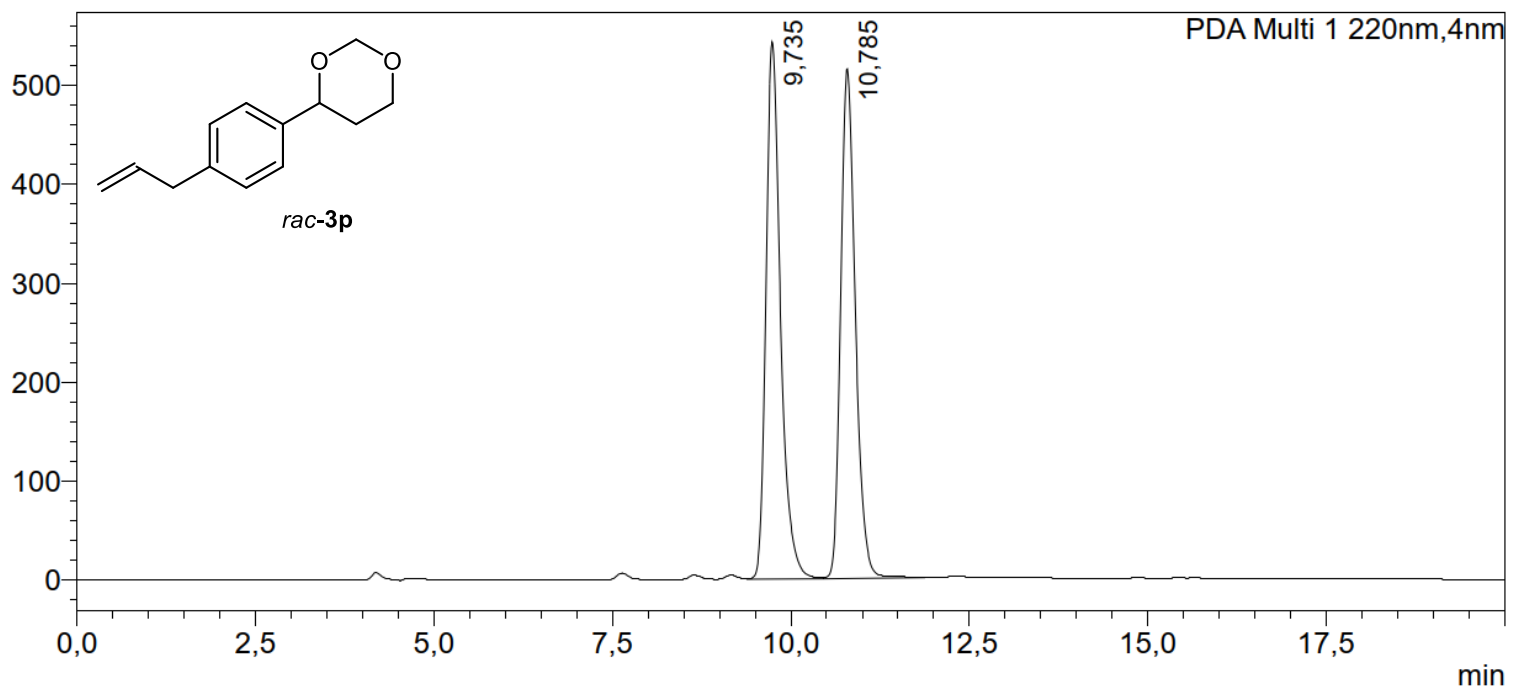

\begin{tabular}{|r|r|r|}
\hline Peak\# & Ret. Time & \multicolumn{1}{c|}{ Area\% } \\
\hline 1 & 9,735 & 50,958 \\
\hline 2 & 10,785 & 49,042 \\
\hline Total & & 100,000 \\
\hline
\end{tabular}

mAU

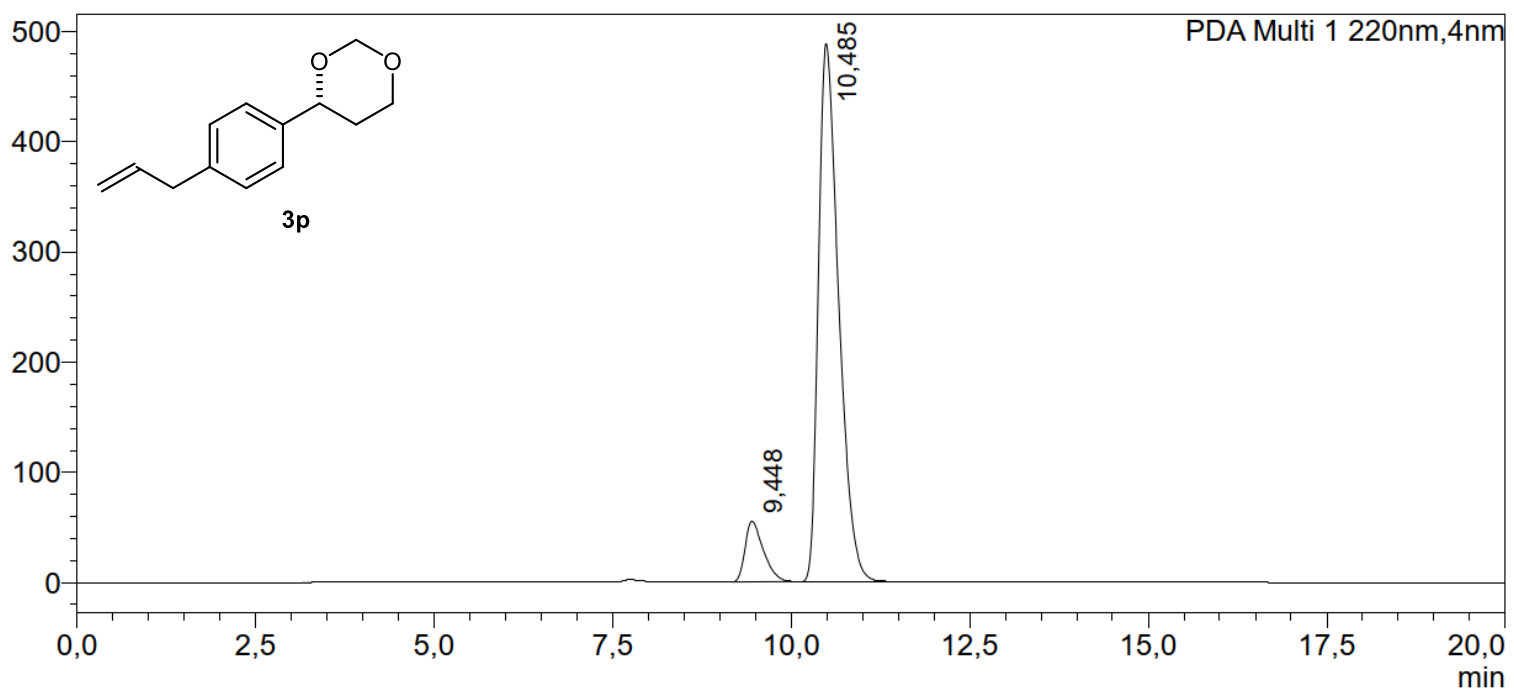

\begin{tabular}{|r|r|r|}
\hline Peak\# & Ret. Time & \multicolumn{1}{|c|}{ Area\% } \\
\hline 1 & 9,448 & 9,220 \\
\hline 2 & 10,485 & 90,780 \\
\hline Total & & 100,000 \\
\hline
\end{tabular}


The Catalytic Asymmetric Intermolecular Prins Reaction Copies of HPLC traces

(Chiralpak IB-3 column, Heptane/i-PrOH 95:5, 1 mL/min, $25^{\circ} \mathrm{C}, 220 \mathrm{~nm}$ )

mAU

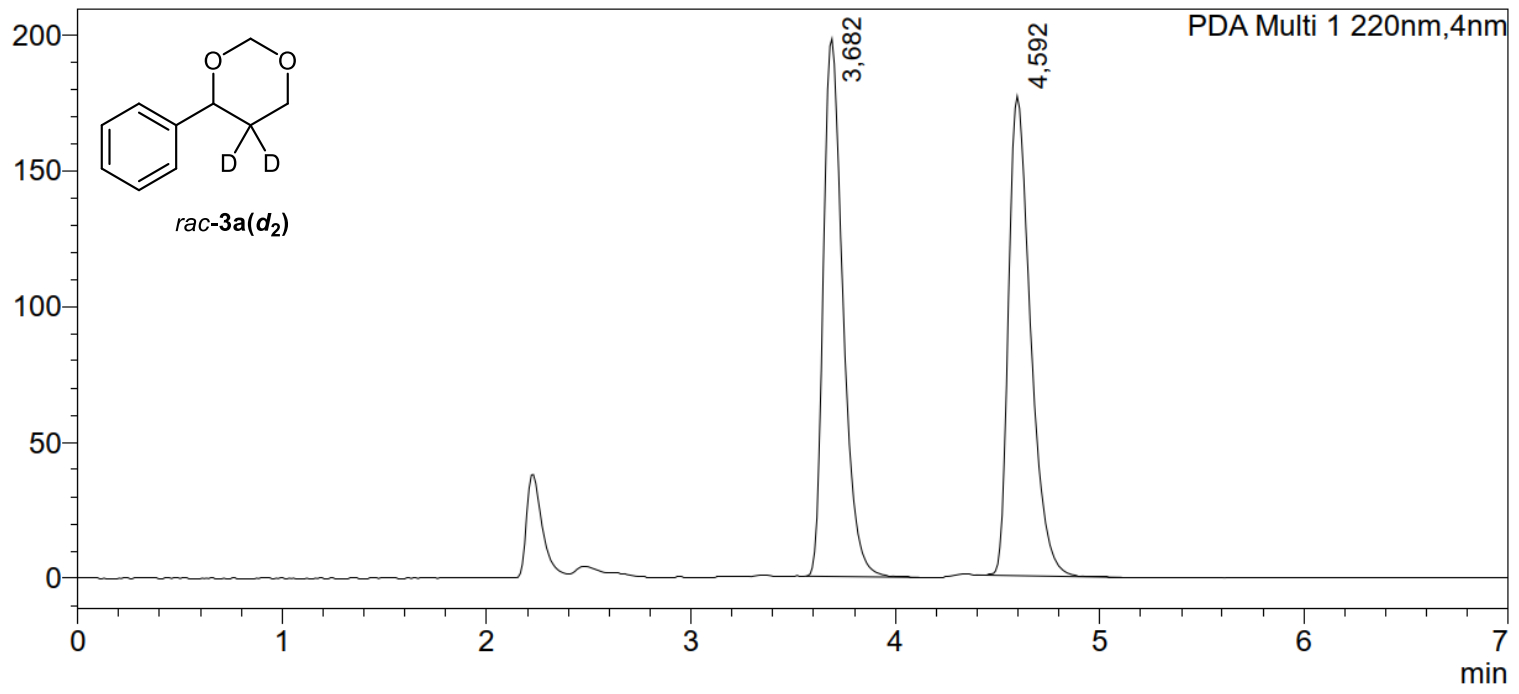

\begin{tabular}{|r|r|r|}
\hline Peak\# & Ret. Time & \multicolumn{1}{c|}{ Area\% } \\
\hline 1 & 3,682 & 49,943 \\
\hline 2 & 4,592 & 50,057 \\
\hline Total & & 100,000 \\
\hline
\end{tabular}

mAU

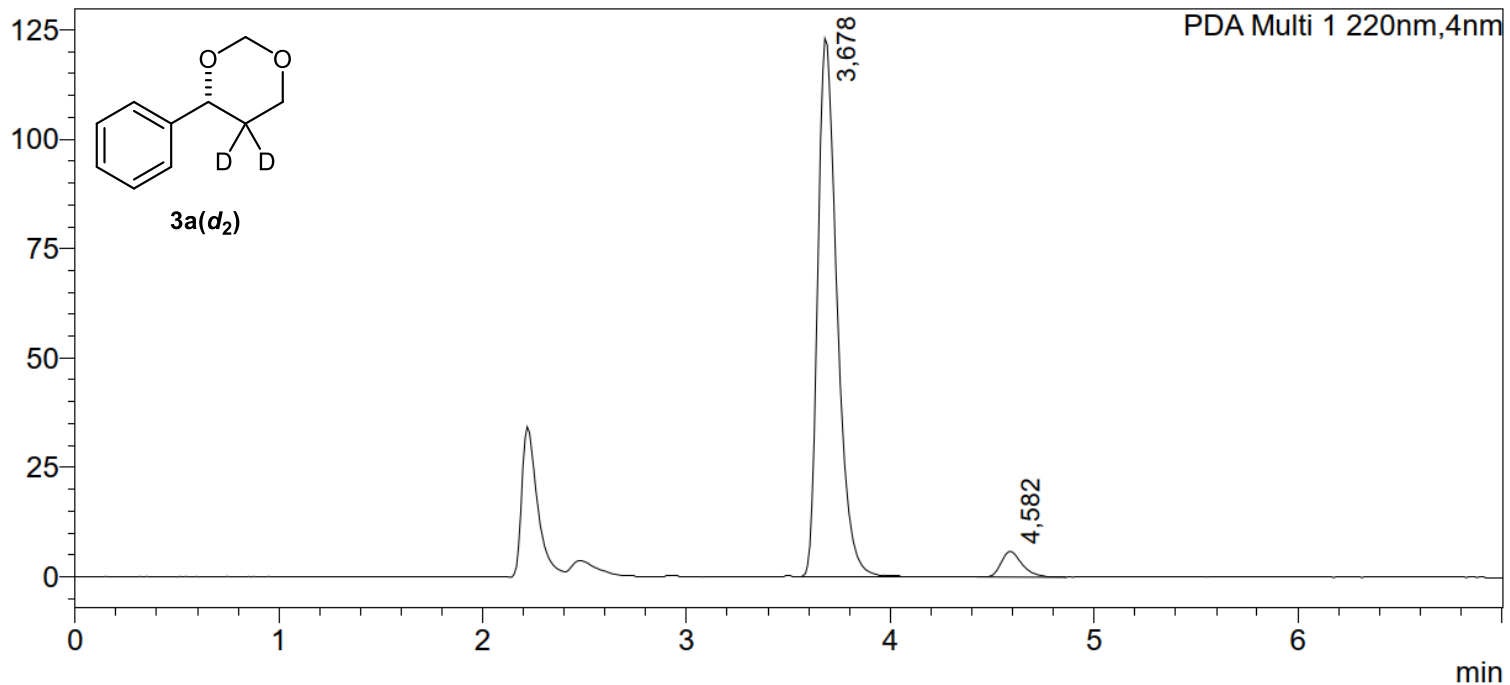

\begin{tabular}{|r|r|r|}
\hline Peak\# & Ret. Time & \multicolumn{1}{|c|}{ Area\% } \\
\hline 1 & 3,678 & 95,071 \\
\hline 2 & 4,582 & 4,929 \\
\hline Total & & 100,000 \\
\hline
\end{tabular}


The Catalytic Asymmetric Intermolecular Prins Reaction Copies of HPLC traces

(Chiralpak IB-3 column, Heptane/i-PrOH 95:5, 1 mL/min, $25^{\circ} \mathrm{C}, 220 \mathrm{~nm}$ )

$\mathrm{mAU}$

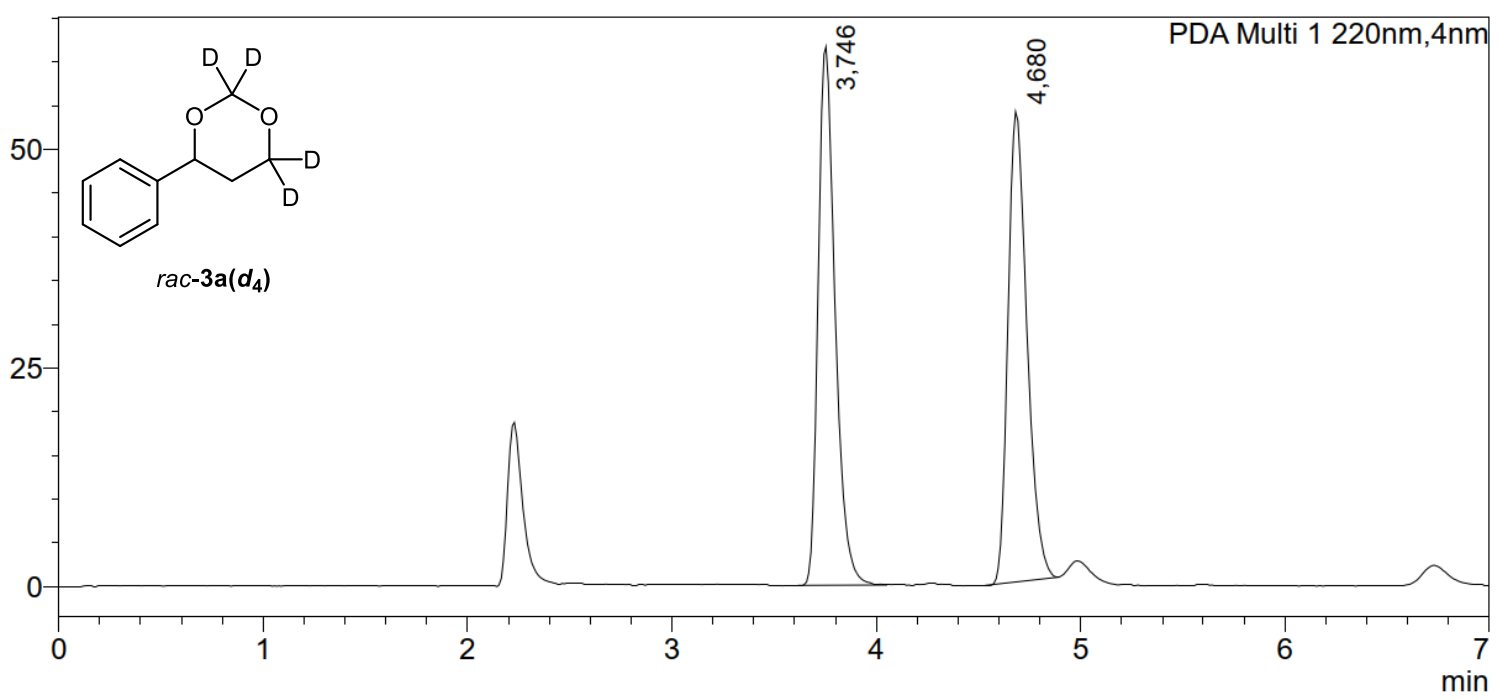

\begin{tabular}{|r|r|r|}
\hline Peak\# & Ret. Time & \multicolumn{1}{c|}{ Area\% } \\
\hline 1 & 3,746 & 50,735 \\
\hline 2 & 4,680 & 49,265 \\
\hline Total & & 100,000 \\
\hline
\end{tabular}

mAU

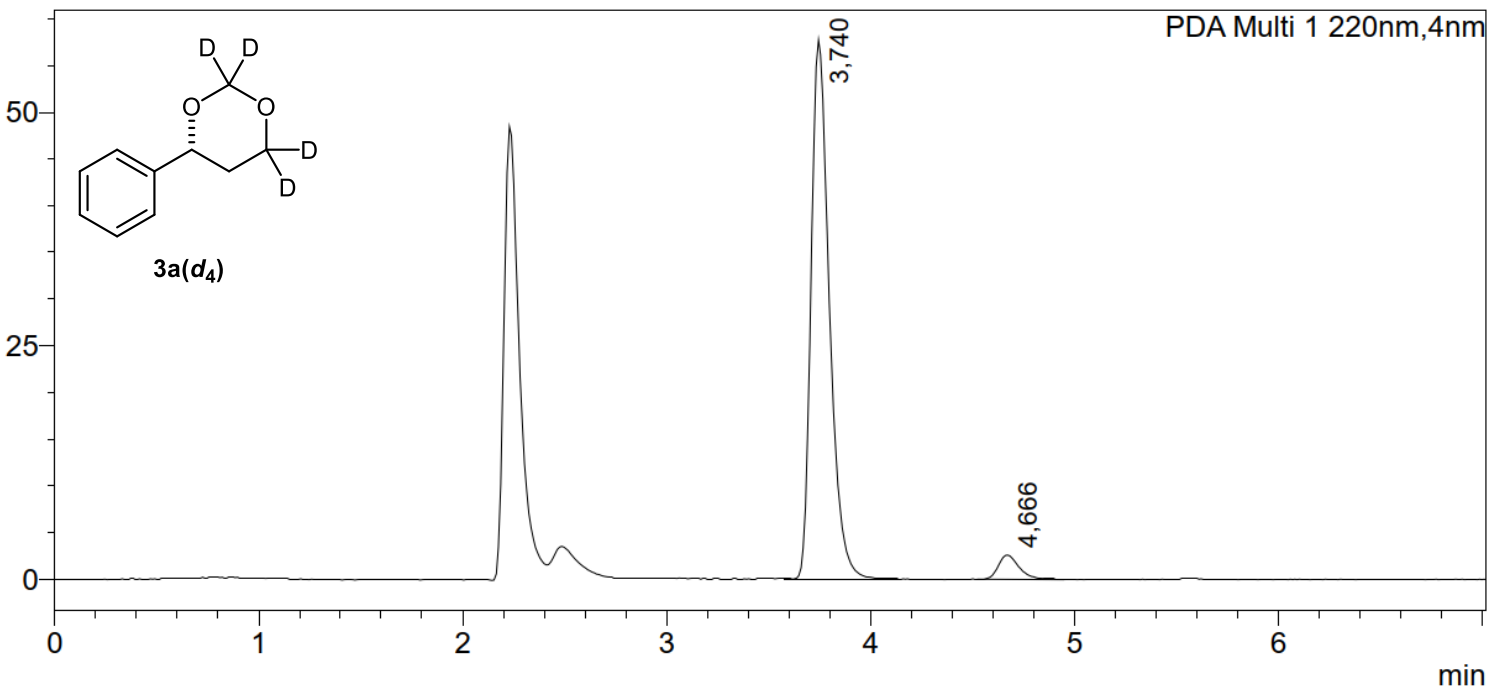

\begin{tabular}{|r|r|r|}
\hline Peak\# & Ret. Time & \multicolumn{1}{c|}{ Area\% } \\
\hline 1 & 3,740 & 95,143 \\
\hline 2 & 4,666 & 4,857 \\
\hline Total & & 100,000 \\
\hline
\end{tabular}


The Catalytic Asymmetric Intermolecular Prins Reaction Copies of HPLC traces

(Chiralpak IB-3 column, Heptane/i-PrOH 95:5, 1 mL/min, $25^{\circ} \mathrm{C}, 220 \mathrm{~nm}$ )

$\mathrm{mAU}$

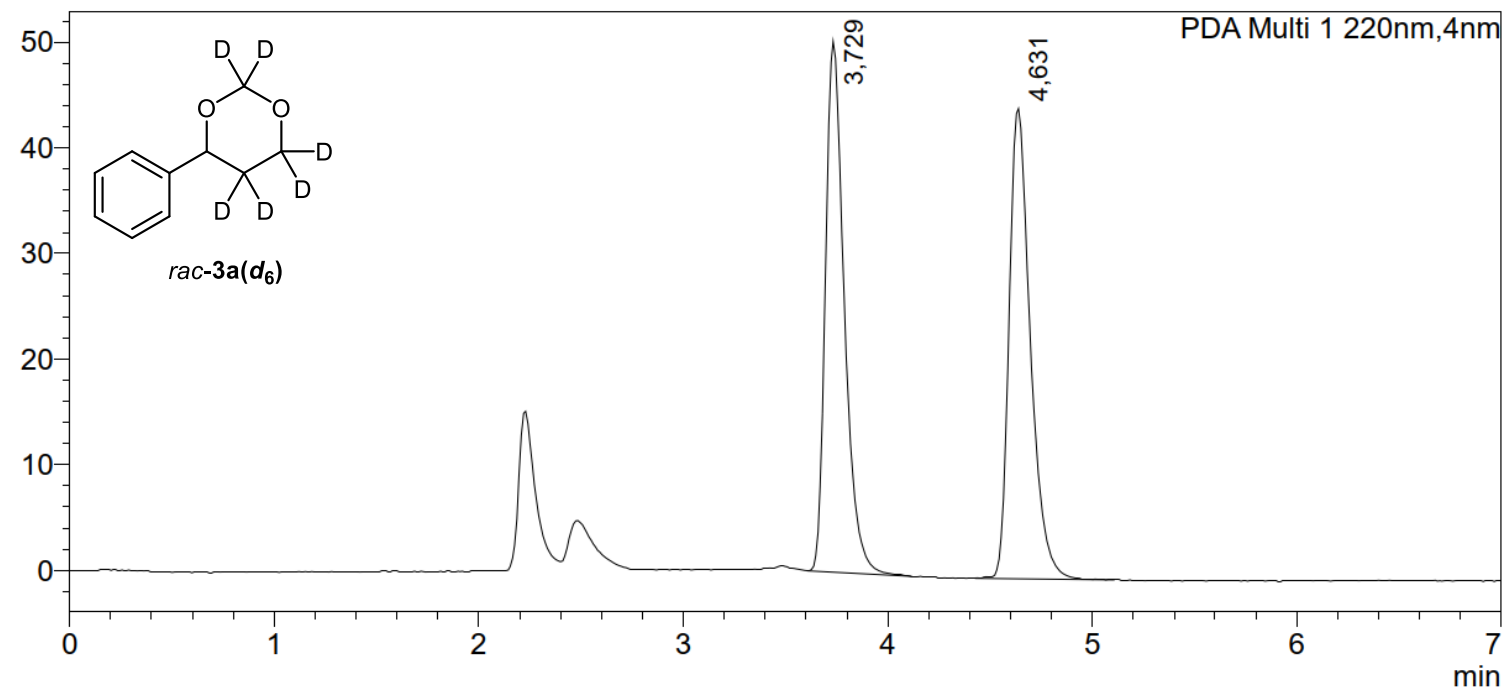

\begin{tabular}{|r|r|r|}
\hline Peak\# & Ret. Time & \multicolumn{1}{c|}{ Area\% } \\
\hline 1 & 3,729 & 50,014 \\
\hline 2 & 4,631 & 49,986 \\
\hline Total & & 100,000 \\
\hline
\end{tabular}

$\mathrm{mAU}$

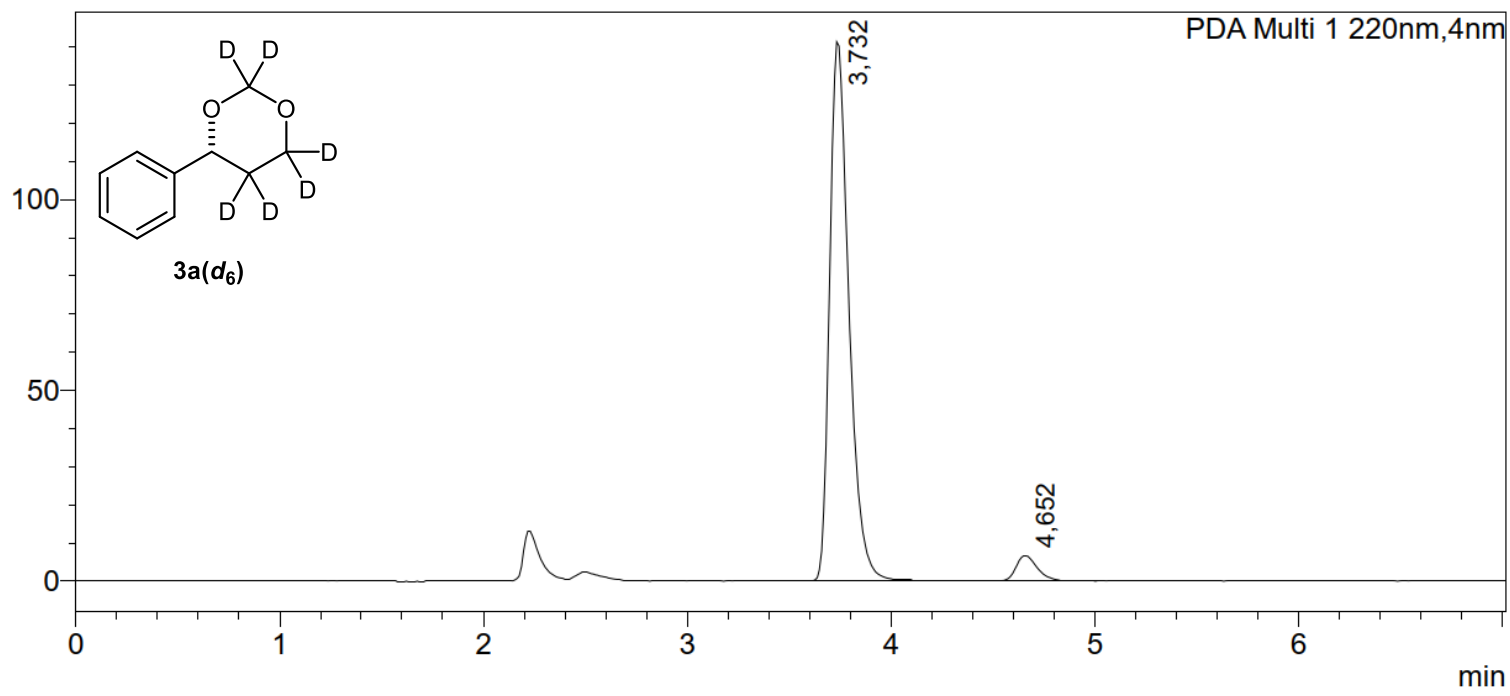

\begin{tabular}{|r|r|r|}
\hline Peak\# & Ret. Time & \multicolumn{1}{c|}{ Area\% } \\
\hline 1 & 3,732 & 95,040 \\
\hline 2 & 4,652 & 4,960 \\
\hline Total & & 100,000 \\
\hline
\end{tabular}


The Catalytic Asymmetric Intermolecular Prins Reaction Copies of HPLC traces

(Chiralpak AD-3 column, Heptane/i-PrOH 97:3, 0.5 mL/min, $25^{\circ} \mathrm{C}, 220 \mathrm{~nm}$ )

mAU

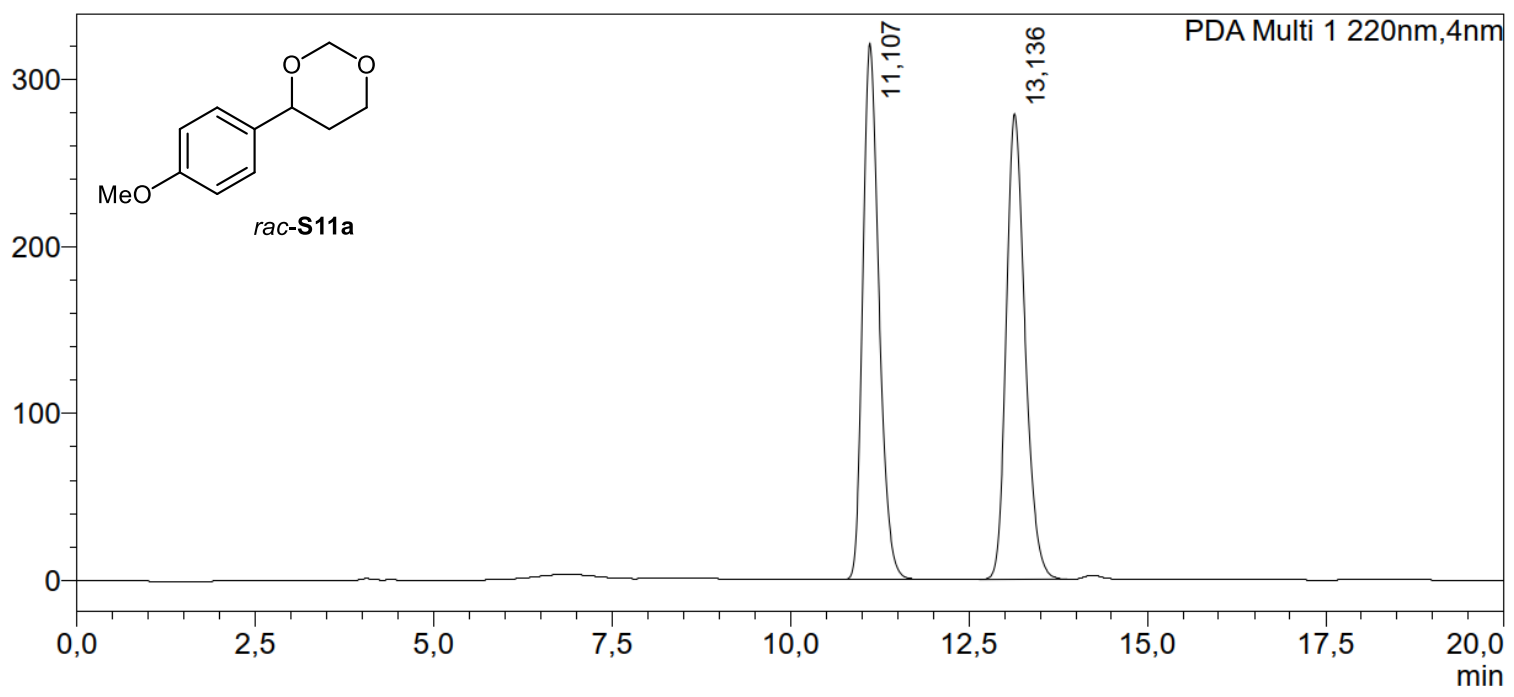

\begin{tabular}{|r|r|r|}
\hline Peak\# & Ret. Time & \multicolumn{1}{c|}{ Area\% } \\
\hline 1 & 11,107 & 49,748 \\
\hline 2 & 13,136 & 50,252 \\
\hline Total & & 100,000 \\
\hline
\end{tabular}

$\mathrm{mAU}$

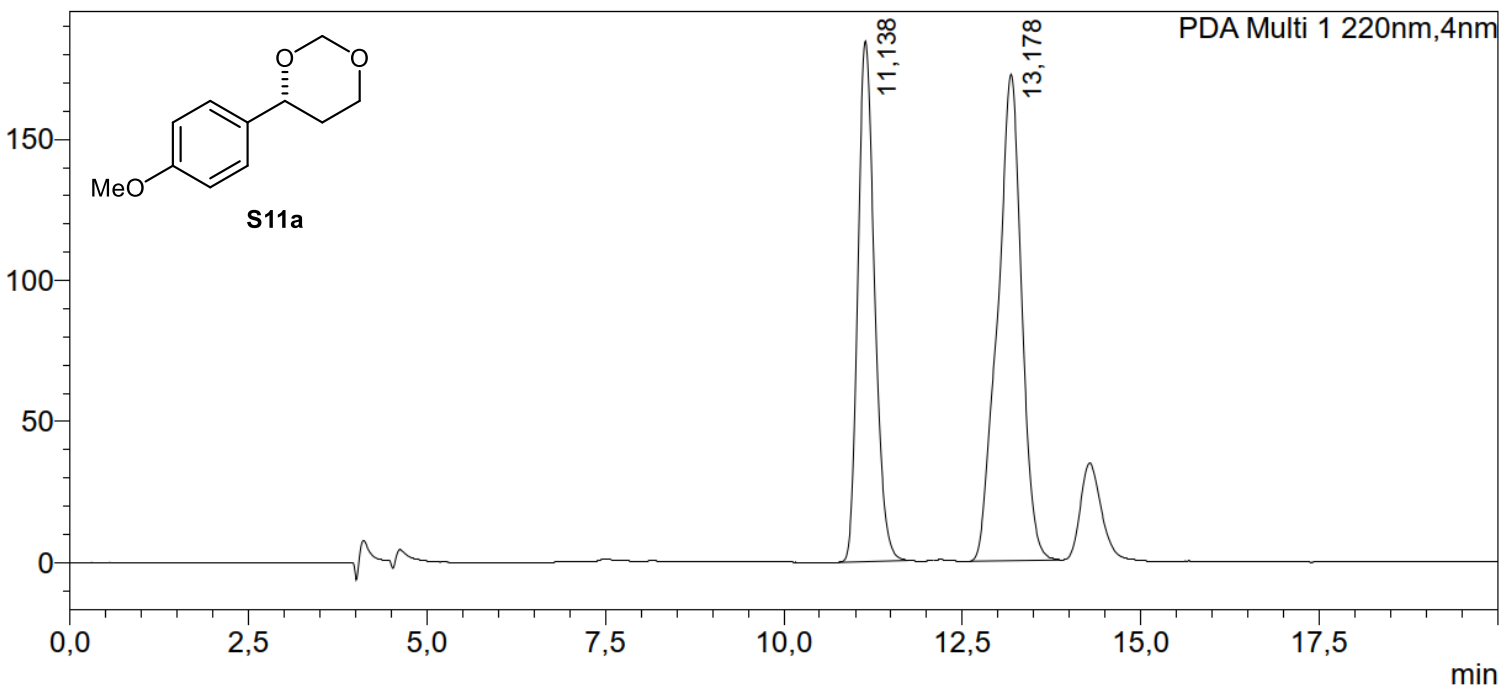

\begin{tabular}{|r|r|r|}
\hline Peak\# & Ret. Time & \multicolumn{1}{|c|}{ Area\% } \\
\hline 1 & 11,138 & 42,616 \\
\hline 2 & 13,178 & 57,384 \\
\hline Total & & 100,000 \\
\hline
\end{tabular}


The Catalytic Asymmetric Intermolecular Prins Reaction Copies of HPLC traces

(Kromasil Amycoat RP column, $\mathrm{CH}_{3} \mathrm{CN} / \mathrm{H}_{2} \mathrm{O} 50: 50,1 \mathrm{~mL} / \mathrm{min}, 25^{\circ} \mathrm{C}, 220 \mathrm{~nm}$ )

$\mathrm{mAU}$

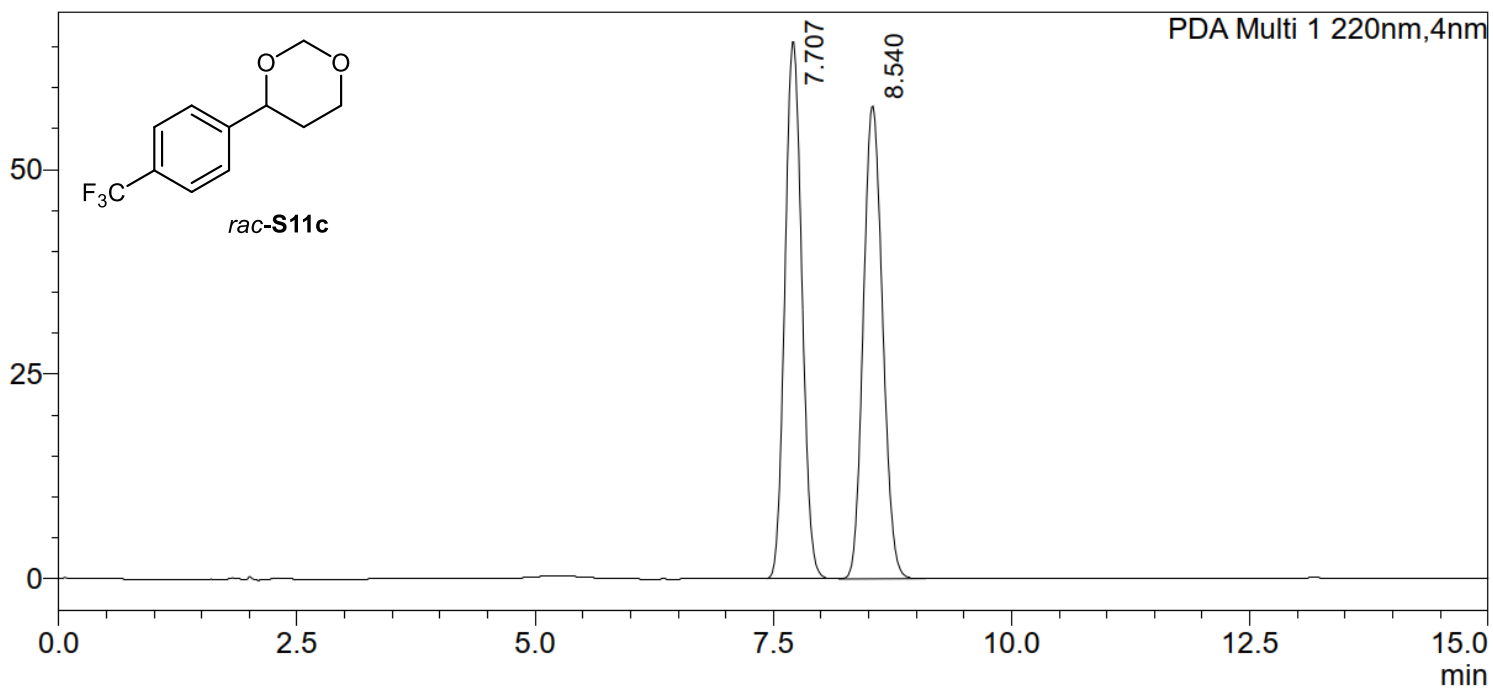

\begin{tabular}{|r|r|r|}
\hline Peak\# & Ret. Time & Area\% \\
\hline 1 & 7.707 & 50.077 \\
\hline 2 & 8.540 & 49.923 \\
\hline Total & & 100.000 \\
\hline
\end{tabular}

mAU

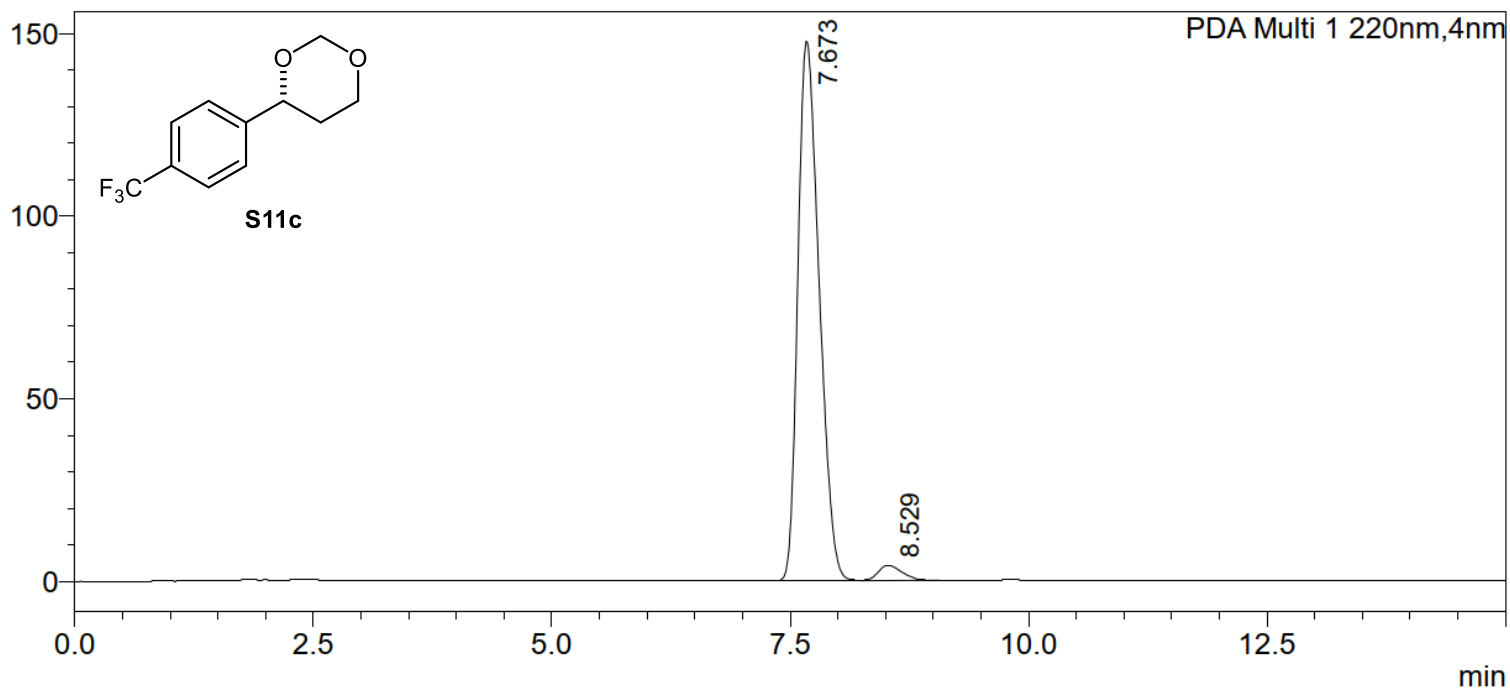

\begin{tabular}{|r|r|r|}
\hline Peak\# & Ret. Time & \multicolumn{1}{c|}{ Area\% } \\
\hline 1 & 7.673 & 96.943 \\
\hline 2 & 8.529 & 3.057 \\
\hline Total & & 100.000 \\
\hline
\end{tabular}


The Catalytic Asymmetric Intermolecular Prins Reaction Copies of HPLC traces

(Chiralpak AD-3 column, Heptane/i-PrOH 95:5, $1 \mathrm{~mL} / \mathrm{min}, 25^{\circ} \mathrm{C}, 220 \mathrm{~nm}$ )

$\mathrm{mAU}$

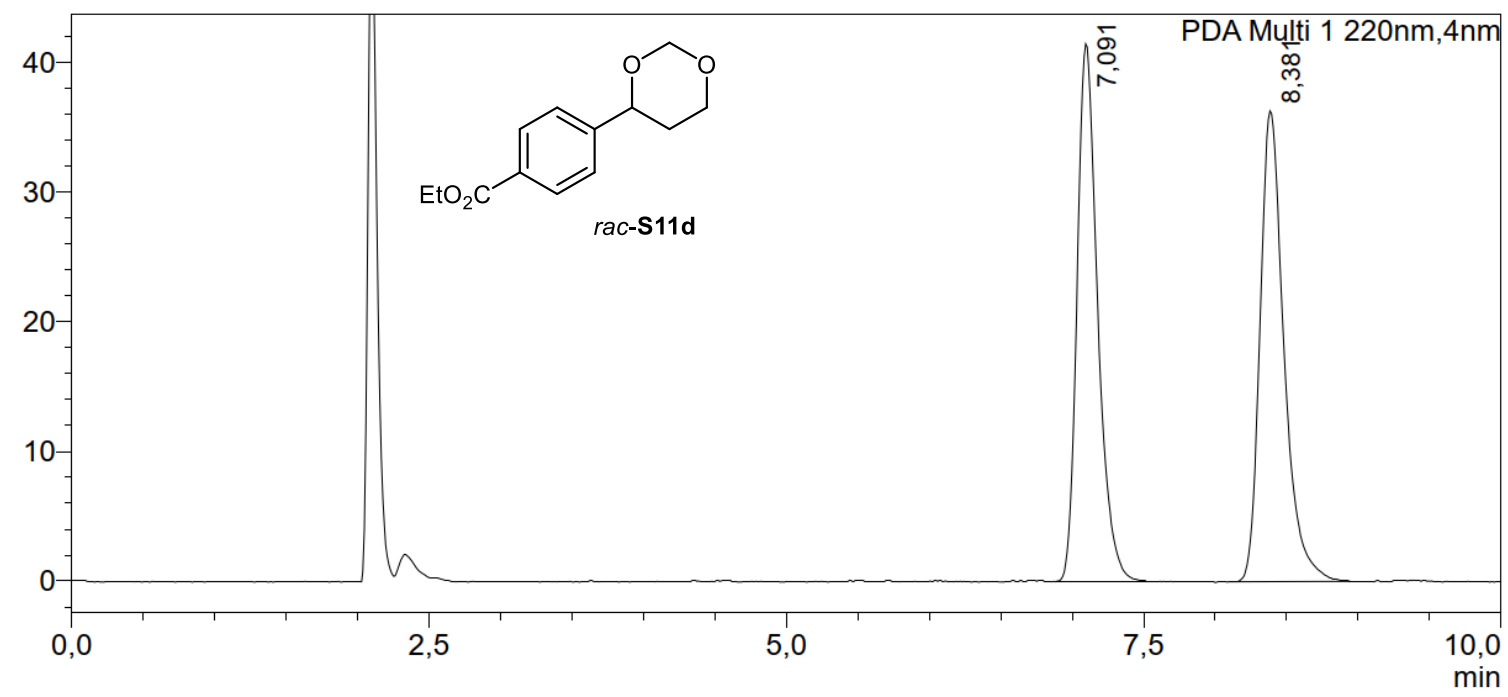

\begin{tabular}{|r|r|r|}
\hline Peak\# & Ret. Time & \multicolumn{1}{c|}{ Area\% } \\
\hline 1 & 7,091 & 49,614 \\
\hline 2 & 8,381 & 50,386 \\
\hline Total & & 100,000 \\
\hline
\end{tabular}

mAU

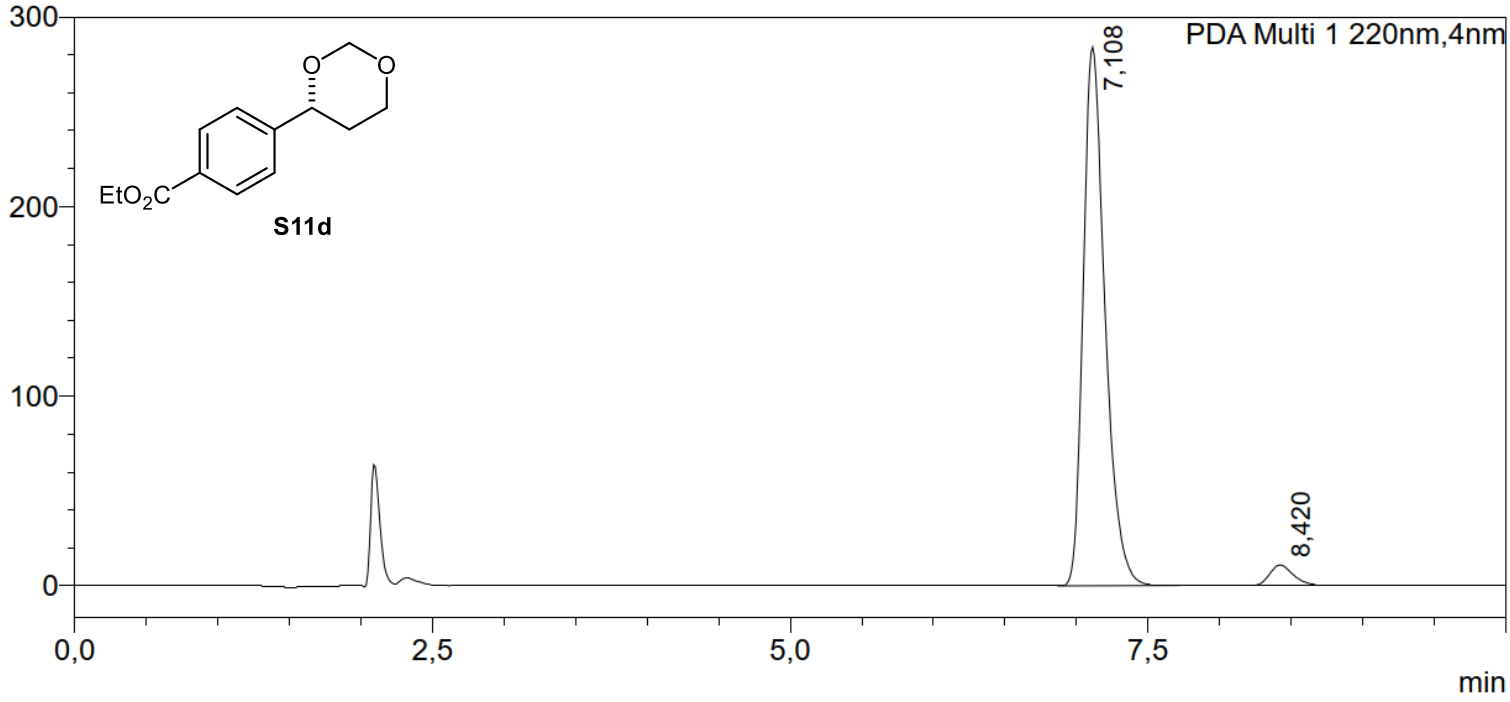

\begin{tabular}{|r|r|r|}
\hline Peak\# & Ret. Time & \multicolumn{1}{c|}{ Area\% } \\
\hline 1 & 7,108 & 95,849 \\
\hline 2 & 8,420 & 4,151 \\
\hline Total & & 100,000 \\
\hline
\end{tabular}


The Catalytic Asymmetric Intermolecular Prins Reaction Copies of HPLC traces

(Chiralpak IC-3 column, Heptane/i-PrOH 97:3, $0.5 \mathrm{~mL} / \mathrm{min}, 25^{\circ} \mathrm{C}, 220 \mathrm{~nm}$ )

mAU

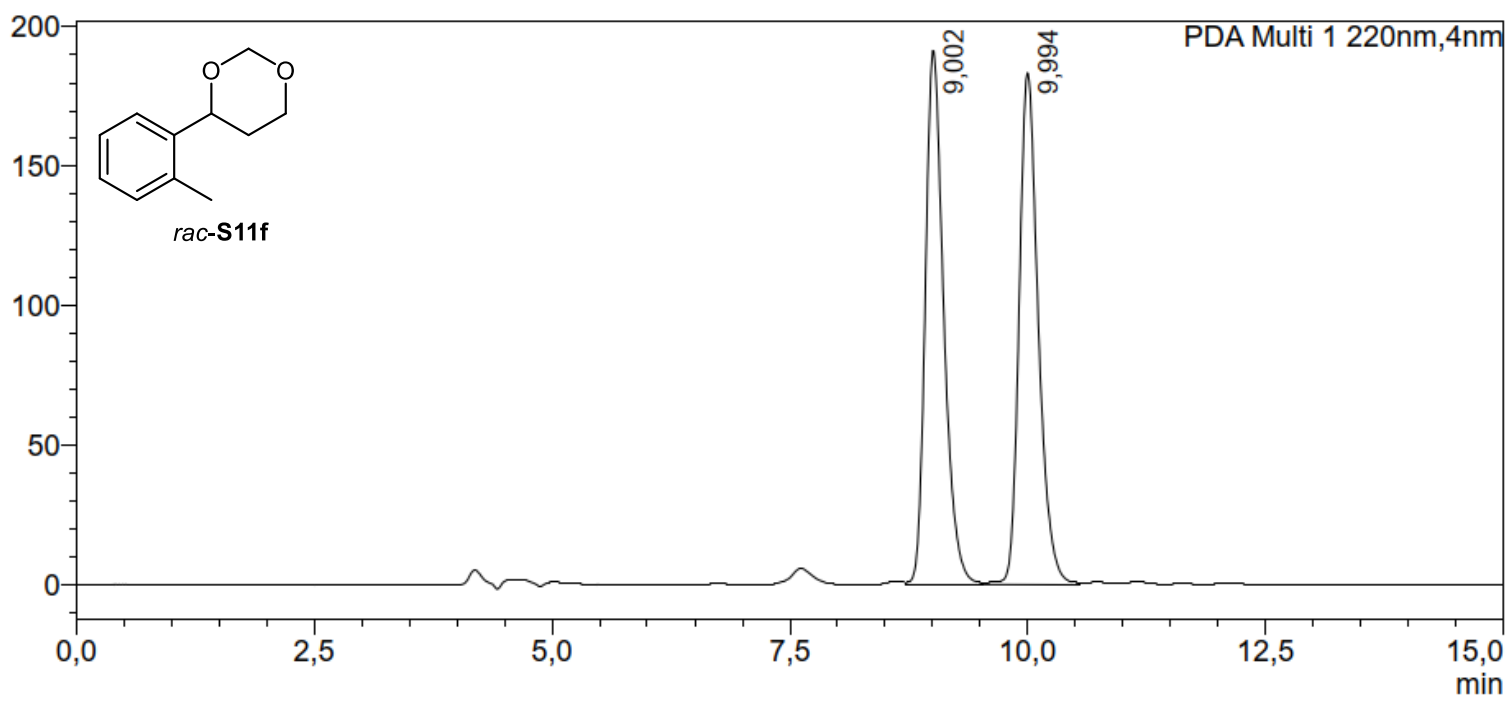

\begin{tabular}{|r|r|r|}
\hline Peak\# & Ret. Time & \multicolumn{1}{c|}{ Area\% } \\
\hline 1 & 9,002 & 49,919 \\
\hline 2 & 9,994 & 50,081 \\
\hline Total & & 100,000 \\
\hline
\end{tabular}

mAU

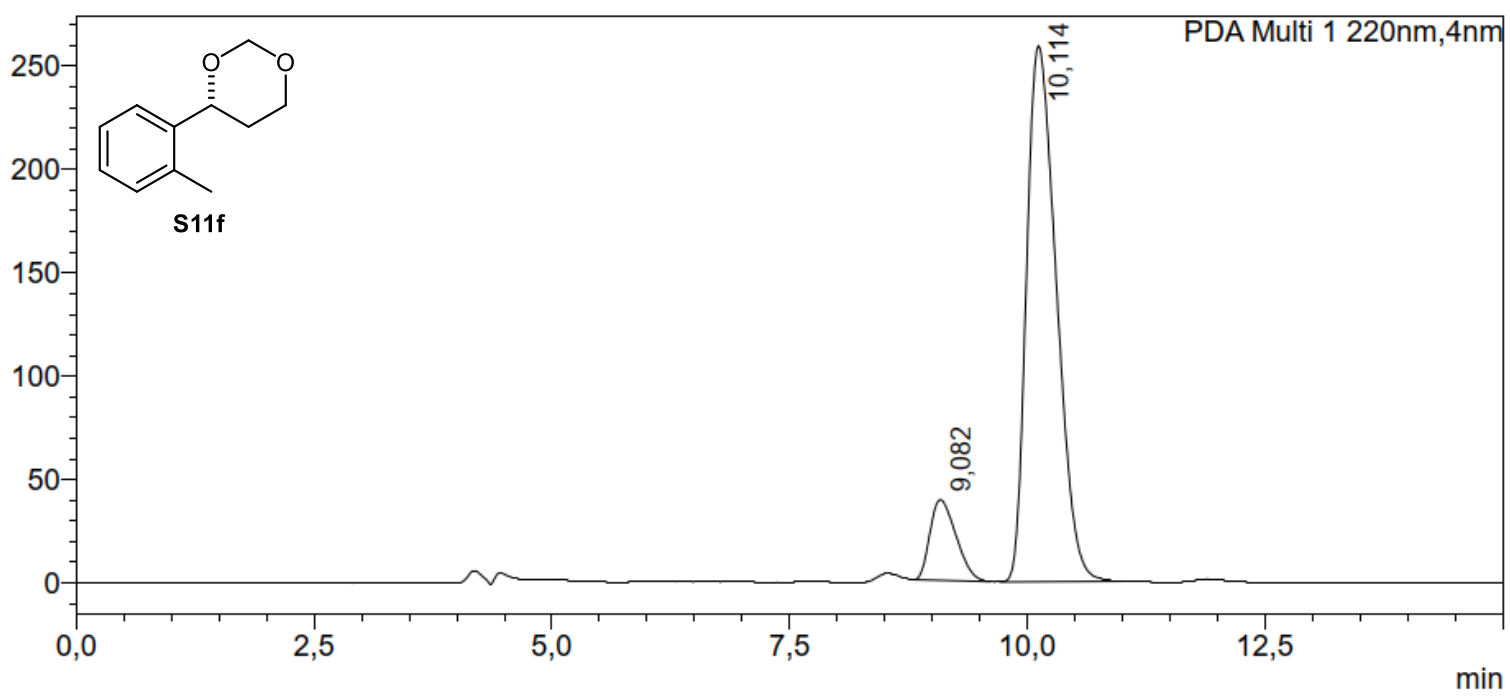

\begin{tabular}{|r|r|r|}
\hline Peak\# & Ret. Time & \multicolumn{1}{c|}{ Area\% } \\
\hline 1 & 9,082 & 11,643 \\
\hline 2 & 10,114 & 88,357 \\
\hline Total & & 100,000 \\
\hline
\end{tabular}


The Catalytic Asymmetric Intermolecular Prins Reaction Copies of HPLC traces

(Chiralpak IC-3 column, Heptane/i-PrOH 95:5, 1 mL/min, $25^{\circ} \mathrm{C}, 220 \mathrm{~nm}$ )

$\mathrm{mAU}$

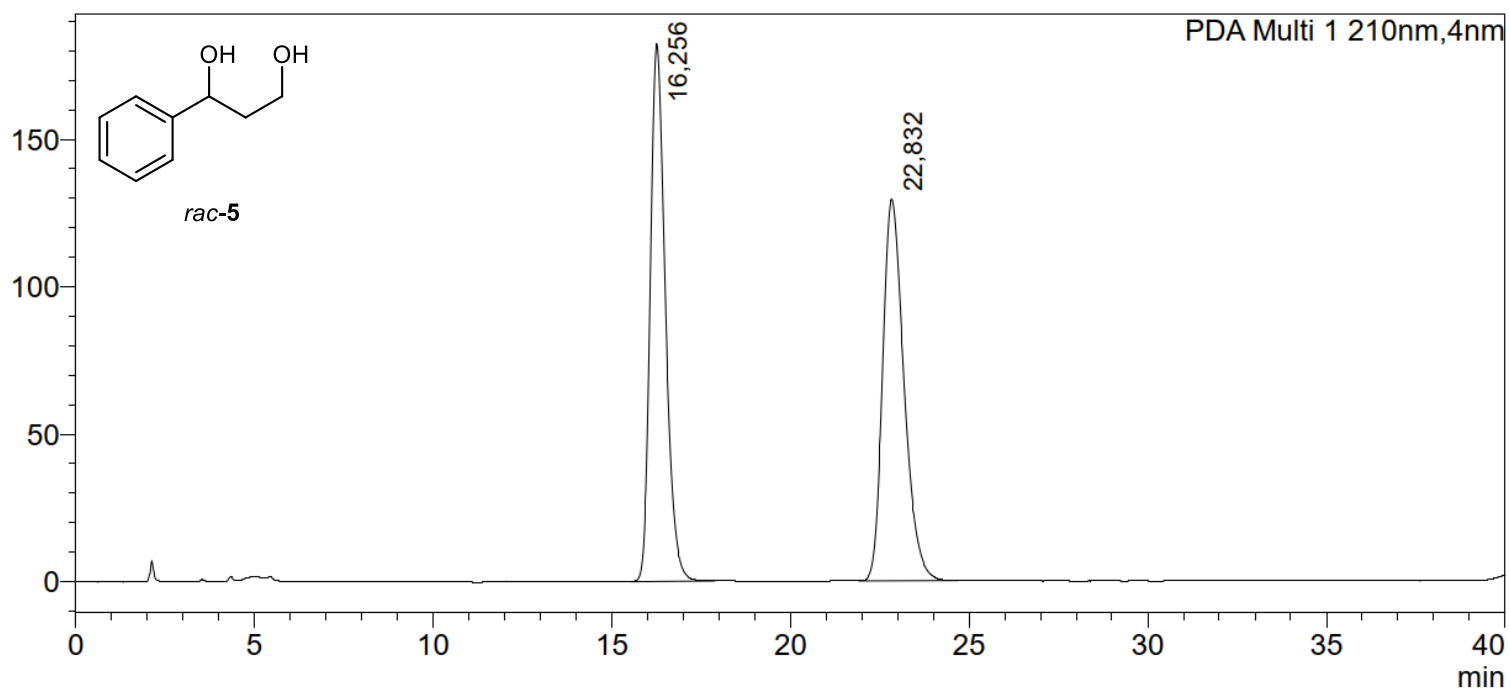

\begin{tabular}{|r|r|r|}
\hline Peak\# & Ret. Time & \multicolumn{1}{c|}{ Area\% } \\
\hline 1 & 16,256 & 49,974 \\
\hline 2 & 22,832 & 50,026 \\
\hline Total & & 100,000 \\
\hline
\end{tabular}

mAU

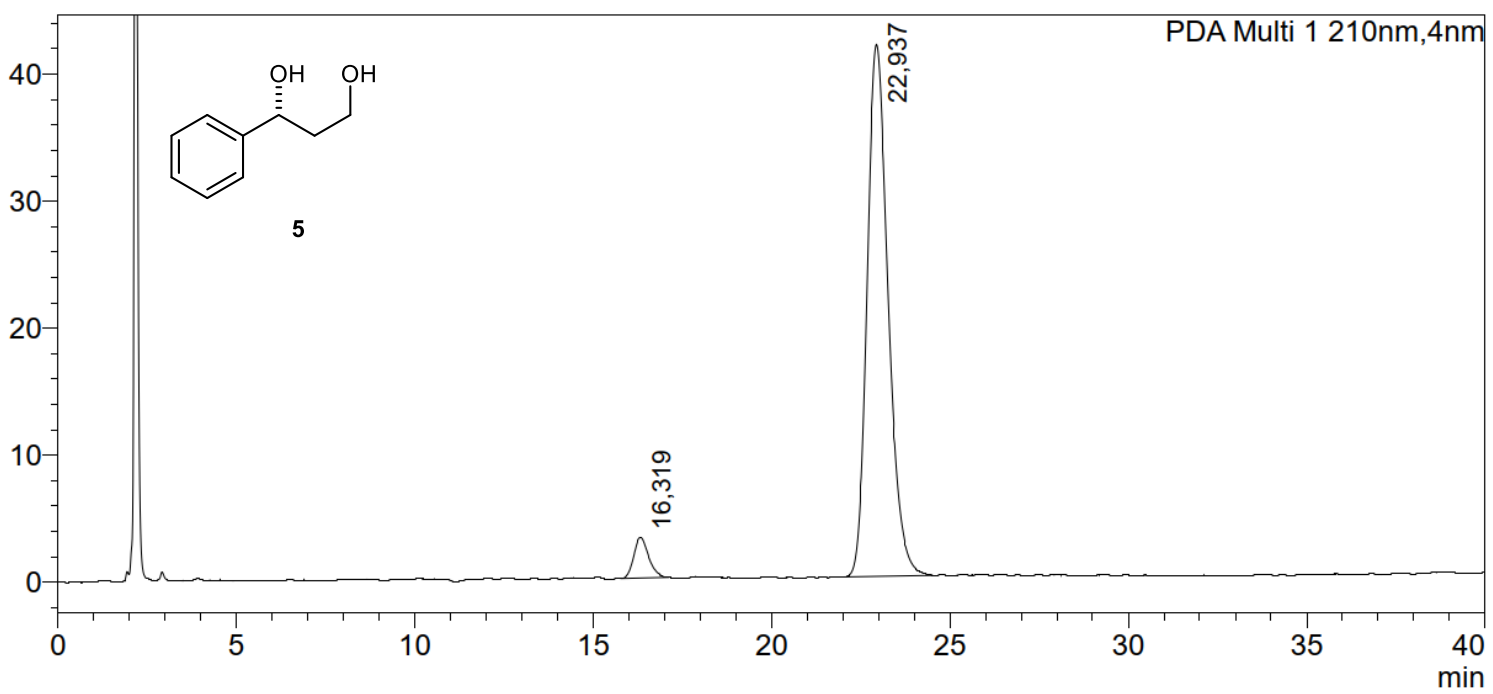

\begin{tabular}{|r|r|r|}
\hline Peak\# & Ret. Time & \multicolumn{1}{|c|}{ Area\% } \\
\hline 1 & 16,319 & 5,133 \\
\hline 2 & 22,937 & 94,867 \\
\hline Total & & 100,000 \\
\hline
\end{tabular}




\section{References}

1. Armarego, W. L. F., Purification of laboratory chemicals. 8th ed.; Butterworth-Heinemann: Woburn, MA, 2003.

2. Harris, R. K.; Becker, E. D.; Cabral de Menezes, S. M.; Granger, P.; Hoffman, R. E.; Zilm, K. W. Further conventions for NMR shielding and chemical shifts (IUPAC Recommendations 2008). Pure Appl. Chem. 2008, 80 (1), 59-84.

3. Dong, X.-Y.; Zhang, Y.-F.; Ma, C.-L.; Gu, Q.-S.; Wang, F.-L.; Li, Z.-L.; Jiang, S.-P.; Liu, X.-Y. A general asymmetric copper-catalysed Sonogashira $\mathrm{C}\left(s p^{3}\right)-\mathrm{C}(s p)$ coupling. Nat. Chem. 2019, 11 (12), 1158-1166.

4. Jiménez-González, L.; García-Muñoz, S.; Álvarez-Corral, M.; Muñoz-Dorado, M.; Rodríguez-García, I. Diastereoselective Synthesis of 2-Aryl-3-vinyl-2,3-dihydrobenzo[b]furans through a Sakurai Reaction: A Mechanistic Proposal. Chem-Eur. J. 2007, 13 (2), 557-568.

5. Al-Hourani, B. J.; Bravo-Vasquez, J. P.; High, L. R. H.; Fenniri, H. Synthesis and characterization of aryl thioacetyl styrene monomers: towards a new generation of SERS-active polymers. Tetrahedron Lett. 2007, 48 (52), 9144-9147.

6. Luo, F.-T.; Xue, C.; Ko, S.-L.; Shao, Y.-D.; Wu, C.-J.; Kuo, Y.-M. Preparation of polystyrene-supported soluble palladacycle catalyst for Heck and Suzuki reactions. Tetrahedron 2005, 61 (25), 6040-6045.

7. Tang, M.; Han, S.; Huang, S.; Huang, S.; Xie, L.-G. Carbosulfenylation of Alkenes with Organozinc Reagents and Dimethyl(methylthio)sulfonium Trifluoromethanesulfonate. Org. Lett. 2020, 22 (24), 9729-9734.

8. Kayaki, Y.; Koda, T.; Ikariya, T. A Highly Effective (Triphenyl phosphite)palladium Catalyst for a Cross-Coupling Reaction of Allylic Alcohols with Organoboronic Acids. Eur. J. Org. Chem. 2004, 2004 (24), 4989-4993.

9. Cívicos, J. F.; Alonso, D. A.; Nájera, C. Oxime Palladacycle-Catalyzed Suzuki-Miyaura Alkenylation of Aryl, Heteroaryl, Benzyl, and Allyl Chlorides under Microwave Irradiation Conditions. Adv. Synth. Catal. 2011, 353 (10), 1683-1687.

10. Kohler, D. G.; Gockel, S. N.; Kennemur, J. L.; Waller, P. J.; Hull, K. L. Palladium-catalysed anti-Markovnikov selective oxidative amination. Nat. Chem. 2018, 10 (3), 333-340.

11. Xiao, J.; Li, Q.; Shen, R.; Shimada, S.; Han, L. B. Phosphonium Phenolate Zwitterion vs Phosphonium Ylide: Synthesis, Characterization and Reactivity Study of a Trimethylphosphonium Phenolate Zwitterion. Adv. Synth. Catal. 2019, 361 (24), 5715-5720.

12. Kapeller, D.; Barth, R.; Mereiter, K.; Hammerschmidt, F. Preparation of Chiral $\alpha-O x y-\left[{ }^{2} H_{1}\right]$ methyllithiums of $99 \%$ ee and Determination of Their Configurational Stability. J. Am. Chem. Soc. 2007, 129 (4), 914-923.

13. Liu, L.; Kaib, P. S. J.; Tap, A.; List, B. A General Catalytic Asymmetric Prins Cyclization. J. Am. Chem. Soc. 2016, 138 (34), 10822-10825.

14. Maegawa, T.; Koutani, Y.; Otake, K.; Fujioka, H. Methylene Acetal Formation from 1,2- and 1,3-Diols Using an O,SAcetal, 1,3-Dibromo-5,5-dimethylhydantoin, and BHT. J. Org. Chem. 2013, 78 (7), 3384-3390.

15. Zhang, J.; Hua, L.; Li, F.; Wu, X.; Tian, S.; Yang, J. Prins Cyclization of Styrenes or Acetophenone Catalyzed by DBSA in Water. Synthetic Commun. 2011, 42 (8), 1234-1242.

16. Kalkhambkar, R. G.; Jeong, Y. T. Highly Efficient Synthesis of 1,3-Dioxanes via Prins Reaction in Brønsted-Acidic Imidazolium Ionic Liquid. Synthetic Commun. 2014, 44 (6), 762-771.

17. Fujioka, H.; Senami, K.; Kubo, O.; Yahata, K.; Minamitsuji, Y.; Maegawa, T. Mild Deprotection of Methylene Acetals in Combination with Trimethylsilyl Triflate-2,2'-Bipyridyl. Chem. Pharm. Bull. 2010, 58 (3), 426-428.

18. Sood, D. E.; Champion, S.; Dawson, D. M.; Chabbra, S.; Bode, B. E.; Sutherland, A.; Watson, A. J. B. Deoxyfluorination with $\mathrm{CuF}_{2}$ : Enabled by Using a Lewis Base Activating Group. Angew. Chem. Int. Ed. 2020, 59 (22), 8460-8463.

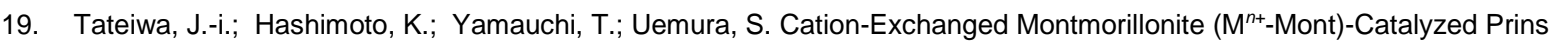
Reaction. B. Chem. Soc. Jpn. 1996, 69 (8), 2361-2368.

20. Adam, J.-M.; Aebi, J.; Binggeli, A.; Green, L.; Hartmann, G.; Maerki, H. P.; Mattei, P.; Ricklin, F.; Roche, O. NOVEL HETEROCYCLYL COMPOUNDS. Patent US 2010/0022518 A1, January 28, 2010.

21. Trofymchuk, S.; Bugera, M. Y.; Klipkov, A. A.; Razhyk, B.; Semenov, S.; Tarasenko, K.; Starova, V. S.; Zaporozhets, O. A.; Tananaiko, O. Y.; Alekseenko, A. N.; Pustovit, Y.; Kiriakov, O.; Gerus, I. I.; Tolmachev, A. A.; Mykhailiuk, P. K. Deoxofluorination of (Hetero)aromatic Acids. J. Org. Chem. 2020, 85 (5), 3110-3124.

22. Garg, G.; Forsberg, L. K.; Zhao, H.; Blagg, B. S. J. Development of Phenyl Cyclohexylcarboxamides as a Novel Class of Hsp90 C-terminal Inhibitors. Chem-Eur. J. 2017, 23 (65), 16574-16585. 


\section{References}

23. Yue, Y.; Turlington, M.; Yu, X. Q.; Pu, L. 3,3'-Anisyl-Substituted BINOL, $\mathrm{H}_{4} \mathrm{BINOL}$, and $\mathrm{H}_{8} \mathrm{BINOL}$ Ligands: Asymmetric Synthesis of Diverse Propargylic Alcohols and Their Ring-Closing Metathesis to Chiral Cycloalkenes. J. Org. Chem. 2009, 74 (22), 8681-8689.

24. Ahmed, I.; Clark, D. A. Rapid Synthesis of 3,3' Bis-Arylated BINOL Derivatives Using a C-H Borylation in Situ SuzukiMiyaura Coupling Sequence. Org. Lett. 2014, 16 (16), 4332-4335.

25. Ishitani, H.; Ueno, M.; Kobayashi, S. Enantioselective Mannich-Type Reactions Using a Novel Chiral Zirconium Catalyst for the Synthesis of Optically Active $\beta$-Amino Acid Derivatives. J. Am. Chem. Soc. 2000, 122 (34), 8180-8186.

26. Schwengers, S. A.; De, C. K.; Grossmann, O.; Grimm, J. A. A.; Sadlowski, N. R.; Gerosa, G. G.; List, B. Unified Approach to Imidodiphosphate-Type Brønsted Acids with Tunable Confinement and Acidity. J. Am. Chem. Soc. 2021, 143 (36), 14835-14844.

27. Neese, F. The ORCA program system. WIREs Comput. Mol. Sci. 2011, 2 (1), 73-78.

28. Zhang, Y.; Yang, W. Comment on "Generalized Gradient Approximation Made Simple". Phys. Rev. Lett. 1998, 80 (4), 890-890.

29. (a) Grimme, S.; Antony, J.; Ehrlich, S.; Krieg, H. A consistent and accurate ab initio parametrization of density functional dispersion correction (DFT-D) for the 94 elements H-Pu. J. Chem. Phys. 2010, 132 (15), 154104; (b) Grimme, S.; Ehrlich, S.; Goerigk, L. Effect of the damping function in dispersion corrected density functional theory. J. Comput. Chem. 2011, 32 (7), 1456-1465.

30. Weigend, F.; Ahlrichs, R. Balanced basis sets of split valence, triple zeta valence and quadruple zeta valence quality for $\mathrm{H}$ to Rn: Design and assessment of accuracy. Phys. Chem. Chem. Phys. 2005, 7 (18), 3297-3305.

31. Barone, V.; Cossi, M. Quantum Calculation of Molecular Energies and Energy Gradients in Solution by a Conductor Solvent Model. J. Phys. Chem. A. 1998, 102 (11), 1995-2001.

32. Zhao, Y.; Truhlar, D. G. The M06 suite of density functionals for main group thermochemistry, thermochemical kinetics, noncovalent interactions, excited states, and transition elements: two new functionals and systematic testing of four M06class functionals and 12 other functionals. Theor. Chem. Acc. 2007, 120 (1-3), 215-241.

33. Bickelhaupt, F. M.; Houk, K. N. Analyzing Reaction Rates with the Distortion/Interaction-Activation Strain Model. Angew. Chem. Int. Ed. 2017, 56 (34), 10070-10086.

34. Legault, C. Y.: CYLview, 1.0b; Université de Sherbrooke, 2009 (http://www.cylview.org).

35. Humphrey, W.; Dalke, A.; Schulten, K. VMD: Visual molecular dynamics. J. Mol. Graphics 1996, 14 (1), 33-38.

36. Kupova, O. Y.; Vakulin, I. V.; Talipov, R. F.; Morozkin, N. D.; Talipova, G. R. Theoretical investigation of the role of formaldehyde dimers in the Prins reaction. React. Kinet. Mech. Cat. 2013, 110 (1), 41-52. 